\title{
Integrodifference Equations in Patchy Landscapes
}

\author{
Jeffrey Musgrave
}

Thesis submitted to the Faculty of Graduate and Postdoctoral Studies in partial fulfillment of the requirements for the degree of Doctor of Philosophy in Mathematics ${ }^{1}$

Department of Mathematics and Statistics

Faculty of Science

University of Ottawa

(c) Jeffrey Musgrave, Ottawa, Canada, 2013

\footnotetext{
${ }^{1}$ The Ph.D. program is a joint program with Carleton University, administered by the OttawaCarleton Institute of Mathematics and Statistics
} 


\section{Abstract}

In this dissertation, we study integrodifference equations in patchy landscapes. Specifically, we provide a framework for linking individual dispersal behavior with populationlevel dynamics in patchy landscapes by integrating recent advances in modeling dispersal into an integrodifference equation.

First, we formulate a random-walk model in a patchy landscape with patchdependent diffusion, settling, and mortality rates. We incorporate mechanisms for individual behavior at an interface which, in general, results in the probability-density function of the random walker being discontinuous at an interface. We show that the dispersal kernel can be characterized as the Green's function of a second-order differential operator and illustrate the kind of (discontinuous) dispersal kernels that arise from our approach. We examine the dependence of obtained kernels on model parameters.

Secondly, we analyze integrodifference equations in patchy landscapes equipped with discontinuous kernels. We obtain explicit formulae for the critical-domain-size problem, as well as explicit formulae for the analogous critical size of good patches on an infinite, periodic, patchy landscape. We examine the dependence of obtained formulae on individual behavior at an interface. Through numerical simulations, we observe that, if the population can persist on an infinite, periodic, patchy landscape, its spatial profile can evolve into a discontinuous traveling periodic wave. We derive a dispersion relation for the speed of the wave and illustrate how interface behavior 
affects invasion speeds.

Lastly, we develop a strategic model for the spread of the emerald ash borer and its interaction with host trees. A thorough literature search provides point estimates and interval ranges for model parameters. Numerical simulations show that the spatial profile of an emerald ash borer invasion evolves into a pulse-like solution that moves with constant speed. We employ Latin hypercube sampling to obtain a plausible collection of parameter values and use a sensitivity analysis technique, partial rank correlation coefficients, to identify model parameters that have the greatest influence on obtained speeds. We illustrate the applicability of our framework by exploring the effectiveness of barrier zones on slowing the spread of the emerald ash borer invasion. 


\section{Acknowledgements}

First and most importantly, I want to thank my advisor Dr. Frithjof Lutscher. Without his guidance and continuous support during my studies and research, this thesis would not have been possible. His open-door policy, patience when discussing advanced mathematical theory, and inspiring discussions on mathematical ecology made my time at the university an enjoyable one. I also wish to acknowledge his financial support through research grants and providing me with the opportunity to attend numerous workshops and conferences.

I am grateful to my thesis committee, Dr. David Amundsen and Dr. Robert Smith?, for taking the time to periodically meet throughout my studies, providing constructive feedback, and for inspiring discussions. I would like to thank them for their willingness to meet with me outside of committee meetings and for providing me with insightful ideas that advanced my research projects. I would also like to thank them, along with Dr. Benoit Dionne, for their careful reading of my thesis. I would also like to thank the members of the mathematical biology/ecology research group at the University of Ottawa for insightful discussions during group meetings.

I would also like to thank the Faculty of Graduate Studies and Postdoctoral Studies and the Department of Mathematics and Statistics at the University of Ottawa for their tremendous financial support during my Ph.D. This research was also partially funded by a postgraduate doctoral scholarship from the National Sciences and Engineering Research Council of Canada. 
Without the love and support of my fiancée, Adrienne, this thesis would not have been possible. Her constant encouragement, and never complaining about spending many nights and weekends alone, made the end goal of finishing this thesis attainable.

Last, but certainly not least, I would like to dedicate this thesis to my parents, Wayne and Gloria Musgrave. Without their constant support, encouragement, and love, this project would have never happened. I am forever indebted to them for many things, too many to list here. 


\section{Dedication}

To my parents Wayne and Gloria, my fiancée Adrienne, my nephews and my nieces. 


\section{Contents}

List of Figures $\quad$ x

List of Tables $\quad$ xiv

1 Introduction 1

1.1 Biological Motivation ................. 1

1.2 Integrodifference Equations . . . . . . . . . . . . . . 3

1.3 Mathematical Analysis of IDEs . . . . . . . . . . . 6

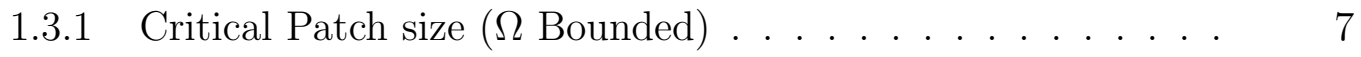

1.3.2 Spreading Speeds and Traveling Waves ( $\Omega$ Unbounded) . . . 11

1.4 Outline of the Thesis . . . . . . . . . . . . 16

2 A Random Walk Model for Individual Dispersal 19

2.1 The Model . . . . . . . . . . . . . . . . . 20

2.2 The Dispersal Kernel . . . . . . . . . . . . . . . . . . . . 21

2.3 Dispersal in Patchy Landscapes . . . . . . . . . . . . . 23

2.4 Dispersal Kernels in Patchy Landscapes . . . . . . . . . . . . . . 26

3 Dispersal Kernels $\quad 29$

$3.1 \quad$ A Single Interface . . . . . . . . . . . . . . . 30

3.2 A Single Patch . . . . . . . . . . . . . . 35

3.3 Several Patches . . . . . . . . . . . . . . . . 41 
3.4 Dispersal Characteristics in Patchy Landscapes . . . . . . . . 45

3.4.1 Dispersal Success in Patchy Landscapes . . . . . . . . . . . . 48

3.4.2 Mean dispersal distance in a patchy landscape . . . . . . . . 52

4 Analysis of Integrodifference Equations: Population Persistence 56

4.1 A Critical Domain Size Problem . . . . . . . . . . . . . 57

4.1.1 Procedure for Obtaining the Dominant Eigenpair _ . . . . . 59

4.1.2 Qualitative Behavior of Critical Domain Size Formulae . . . 62

$4.2 \quad$ A Periodic Landscape . . . . . . . . . . . . . . . . 65

4.3 Stability Equivalence of Operators . . . . . . . . . . . . 73

4.4 Application: Critical Size of Good Patches . . . . . . . . . . . 76

4.4.1 Qualitative Behavior of Critical Size of Good Patches . . . . 80

4.5 Effect of Relevant Scales on the Critical Size of Good Patches . . 83

5 Analysis of Integrodifference Equations...

5.1 Traveling Periodic Waves . . . . . . . . . . . . . . . 90

5.2 Dispersion Relation for a Traveling Periodic Wave . . . . . . . . 97

5.3 Analysis of Dispersion Relation . . . . . . . . . . . . . . 101

6 The Interplay between Individual Dispersal Mechanisms and Barrier Zones: A Case Study on the Emerald Ash Borer 108

6.1 Introduction . . . . . . . . . . . . . . . . . . . . . . . 108

6.2 A Strategic Model for the Emerald Ash Borer . . . . . . . . . . . 110

6.2.1 Parameter Estimation . . . . . . . . . . . . . . 112

6.3 Homogeneous Landscape . . . . . . . . . . . . . . . . . . 115

6.4 A Periodic Landscape . . . . . . . . . . . . . . . . . . 122

6.4.1 Homogeneous Dispersal . . . . . . . . . . . . . . . . 122

6.4.2 Heterogenous Dispersal . . . . . . . . . . . . . 129 
7 Discussion

A Table of Variables, Parameters, Important Quantities, and Notation

B Interchange of Limit and Integral Operator

D Existence of Dominant Eigenvalue for the Critical Domain Size Problem

E Results on Integral Operators

F Numerical Methods 


\section{List of Figures}

1.1 Critical patch size as a function of the reproductive rate . . . . . . . 10

1.2 Simple traveling wave with constant and accelerating speed; location of wavefront . . . . . . . . . . . . . . . . . . 17

3.1 Dispersal kernels with a single interface for Case $M$ as a function of

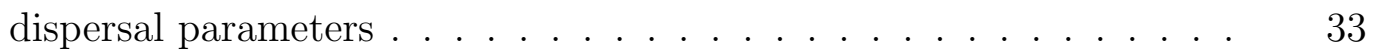

3.2 Dispersal kernels with a single interface for Cases M, S, and C as a function of motility . . . . . . . . . . . . . . 35

3.3 Dispersal kernel in a single bounded patch for Case M . . . . . . . . 38

3.4 Dispersal kernels in a single bounded patch for Case M without and with movement bias . . . . . . . . . . . . . . . . . . . . . 38

3.5 Dispersal kernels in a single bounded patch comparing Cases M, S, and $\mathrm{C} \ldots \ldots \ldots \ldots \ldots$

3.6 Depiction of patchy landscape for Section $3.3 \ldots \ldots$. . . . . . . 42

3.7 Dispersal kernels in patchy landscape as a function of motility and movement bias . . . . . . . . . . . . . . . . . .

3.8 Dispersal success function in a single bounded patch as a function of movement bias, release point, and length of good patch . . . . . .

3.9 Average dispersal success in a single patch as a function of movement

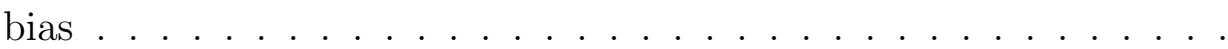


3.10 Average dispersal success in a patchy landscape as function of movement bias . . . . . . . . . . . . . . . . . . . 52

3.11 Mean dispersal distance in a patchy landscape . . . . . . . . . . 54

3.12 Mean dispersal distance in a patchy landscape as a function of movement bias . . . . . . . . . . . . . . . . . . . . . . . . .

3.13 Comparison of kernels in a patchy landscape with equal mean dispersal distance . . . . . . . . . . . . . . . . . . .

4.1 Critical domain size as a function of dispersal parameters with constant motility . . . . . . . . . . . . . . . . 64

4.2 Critical domain size as a function of motility in a bad and good patch 65

4.3 Critical domain size as a function of movement bias . . . . . . . 66

4.4 Critical size of good patches as a function of motility in bad and good patches ........................... 81

4.5 Critical size of good patches as a function of movement bias . . . . . 82

4.6 Critical size of good patches with respect to mortality . . . . . . . 83

4.7 Persistence in a periodic landscape as a function of landscape fragmentation, habitat heterogeneity, and movement bias . . . . . . 87

4.8 Persistence in a periodic landscape as a function of landscape frag-

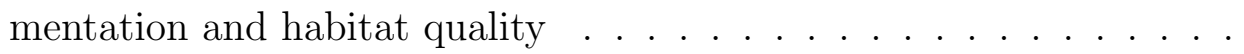

5.1 Discontinuous traveling periodic wave for Case $\mathrm{M}$ and location of

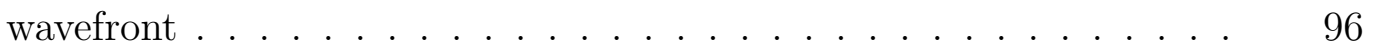

5.2 Numerical Solution of the constant one function with a discontinuous kernel . . . . . . . . . . . . . . . . . . . . . . . . . . 97

5.3 Comparison of numerical and linearized spreading speeds . . . . . 103

5.4 Linearized spreading speed as a function of movement rate in bad

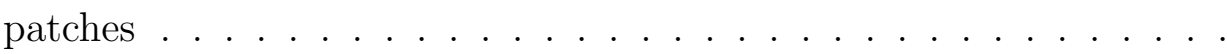


5.5 Linearized spreading speed as a function of movement rate in good

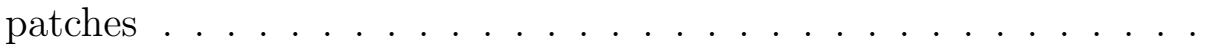

5.6 Linearized spreading speed as a function of movement bias at an

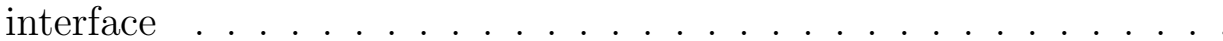

6.1 Numerical solution of non-spatial model showing the number of eggs deposited, remaining phloem levels and cumulative adult emergence

6.2 Numerical solution of EAB spatial model showing spatial profile of a typical invasion and remaining phloem densities . . . . . . . . 117

6.3 PRCCs for spreading speed in a homogeneous landscape . . . . . . .

6.4 Comparison of numerical and linearized spreading speed and their dependence on maximum intrinsic growth rate; numerical speed as a function of overwintering survival of larvae . . . . . . . . .

6.5 PRCCs for pulse width in a homogeneous landscape . . . . . . . . .

6.6 Pulse width as a function of maximum intrinsic growth rate and dependence on overwintering survival of larvae . . . . . . . . .

6.7 Spatial profile of EAB into a periodic landscape of host trees and remaining phloem densities assuming individual dispersal behavior is not patch-dependent $\ldots \ldots \ldots \ldots \ldots \ldots$

6.8 Spatial profile of EAB invasion after 30 generations and location of wavefront in a periodic landscape of host trees assuming individual dispersal behavior is not patch-dependent . . . . . . . . . .

6.9 PRCCs for spreading speed in a periodic landscape of host trees assuming individual dispersal behavior is not patch-dependent . . .

6.10 Numerical spreading speed as a function of maximum intrinsic growth rate and landscape fragmentation; relative reduction in spreading speed as a function of landscape fragmentation and overwintering survival of larvae . . . . . . . . . . . . . . . . . . . . . . 
6.11 Spatial profile of EAB invasion and remaining phloem densities in a periodic landscape of host trees for Cases $\mathrm{C}$ and $\mathrm{M}$. . . . . . . .

6.12 Spatial profile of EAB invasion for 30 generations and location of wavefront . . . . . . . . . . . . . . . .

6.13 Spatial profile of EAB invasion for 30 generations with a strong movement bias towards good patches and location of wavefront . . .

6.14 Relative reduction in spreading speed as a function of effective motility coefficient and landscape fragmentation . . . . . . . . . .

6.15 Relative reduction in spreading speed as a function of movement bias and landscape fragmentation . . . . . . . . . . . .

F.1 Comparison of FFT and Simpson's Rule . . . . . . . . . . . 


\section{List of Tables}

6.1 Parameter estimates for non-spatial EAB model . . . . . . . . 116 


\section{Chapter 1}

\section{Introduction}

\subsection{Biological Motivation}

Invasive species are a class of alien species that are introduced to an area outside their natural environment. Some of these species have the ability to spread rapidly in the new environment, causing environmental and industrial damage. For example, the emerald ash borer (EAB), Agrilus planipennis (Coleoptera: Buprestidae), is an invasive forest insect that was introduced in Detroit, Michigan in 2002 (Poland and McCullough, 2006). EAB is native to Asia and is not considered a threat there because ash trees in Asia appear to be more resistant compared to ash trees in North America (Poland and McCullough, 2006). Since its introduction, the beetle has spread to sixteen other states, has moved into Canada as well, and was found in Ottawa in 2009. This invasion has resulted in the deaths of millions of ash trees, with an estimated removal cost of at least twenty billion dollars (Cappaert et al., 2005).

Much of the research on invasive forest insects has focused on characterizing life cycles, spread rates and evaluation of control strategies (e.g. see Liebhold and Tobin (2008)). Many of these studies have emphasized the importance of (rare) long-distance dispersal events that result in the establishment of satellite populations 
and accelerating rates of spread (Clark, 1998; Bossenbroek et al., 2001; Clark et al., 2001; With, 2002; Muirhead et al., 2006; Prasad et al., 2010). However, since control strategies focus on satellite populations in an effort to slow the spread, a better understanding of local dispersal behavior in these satellite populations is required (Mercader et al., 2009, 2011b; McCullough and Mercader, 2012).

Natural landscapes become increasingly fragmented, and habitats are comprised of favorable and unfavorable patches. Thus, the success of a particular invasion and the resulting rate of spread depend on how individuals make use of available resources, move through the existing landscape and, particularly, how individuals react to interfaces between different landscape types. It is, however, not so clear how specific mechanisms for individual dispersal and behavior at an interface affect persistence and spread. Due to the serious nature of these invasions, it is imperative to gain a better understanding of the mechanisms associated with dispersal and growth so that future invasions may be controlled.

In this dissertation, we employ mathematical models to study the population dynamics and spatial spread of a newly introduced invasive species in fragmented landscapes. In particular, we focus on the behavior of individuals at landscape edges and provide a framework for evaluating the effects and relative importance of different local dispersal mechanisms by integrating recent advances in modeling dispersal into continuous-space, discrete-time population-dynamic models known as integrodifference equations. In the next section, we introduce integrodifference equations and review some analytic techniques that are important for later work in this dissertation. For the convenience of the reader, a summary of notation and symbols used in this dissertation is provided in Appendix A and a summary of numerical methods is provided in Appendix F. 


\subsection{Integrodifference Equations}

Integrodifference equations (IDE) are the discrete-time analogues of reaction-diffusion equations. These equations naturally incorporate the separate growth and dispersal phases that are present in many populations with non-overlapping, synchronous generations throughout a year or generation (Kot and Schaffer, 1986). To formulate an IDE, one assumes that all growth occurs during the sedentary stage and all movement occurs during the dispersal stage. Furthermore, one supposes that growth may be density-dependent but dispersal is not. The growth phase is described by some nonnegative function $f$, and the dispersal phase by a dispersal kernel, $k(x, y)$, where the product $k(x, y) \Delta x$ gives the probability that an individual who started its dispersal process at $y$ will settle in $[x, x+\Delta x)$ (Neubert et al., 1995). The population density in the next generation is obtained by tallying arrivals at location $x$ from all possible locations $y$, or mathematically as the integral operator

$$
N_{t+1}(x)=Q\left[N_{t}\right](x):=\int_{\Omega} k(x, y) f\left(N_{t}(y), y\right) d y,
$$

where $N_{t}(x)$ denotes the density of the population at time or generation $t$ at location $x$, and $\Omega$ is a biological region of interest (Kot and Schaffer, 1986). Note that, by definition, the dispersal kernel is nonnegative for all $x, y \in \Omega$. For the analysis presented in this chapter, it is assumed that the kernel is also continuous.

For the moment, we neglect dispersal and consider the following difference equation given by

$$
N_{t+1}=f\left(N_{t}\right) .
$$

We are interested in the fixed points of equation (1.2.2), which satisfy the equation $N^{*}=f\left(N^{*}\right)$, and their stability. The stability of fixed points of (1.2.2) is studied by applying a small perturbation to the fixed point $N^{*}$, e.g. $N_{t}=N^{*}+\xi_{t}$. Substituting this expression into (1.2.2), expanding the right hand side in a Taylor series about 
$N^{*}$, and neglecting terms of $\mathcal{O}\left(\xi_{t}^{2}\right)$, we obtain

$$
\xi_{t+\tau}=f^{\prime}\left(N^{*}\right) \xi_{t}
$$

Therefore, we see that an equilibrium point is stable if $\left|f^{\prime}\left(N^{*}\right)\right|<1$ and unstable if $\left|f^{\prime}\left(N^{*}\right)\right|>1$. In the case that $\left|f^{\prime}\left(N^{*}\right)\right|=1$, the linearization technique provides us with no conclusion about the stability of $N^{*}$.

Throughout this dissertation, it is required that the growth function satisfy the following technical requirements:

F1: $f$ is continuously differentiable;

F2: $f$ is bounded;

F3: $f^{\prime}(N) \geq 0$ for all $N>0$ and $f$ possess a unique fixed point, $N^{*}$;

F4: $f(N) \leq f^{\prime}(0) N \equiv r_{0} N$ for all $N>0$.

Together these assumptions imply that $f$ is monotone increasing and bounded, as well as bounded by its linearization at zero. Biologically, these assumptions imply that growth functions with overcompensation (e.g. Ricker function) or an Allee effect are excluded from our analysis.

In a homogeneous landscape, a commonly used growth function that satisfies the above technical requirements is the Beverton-Holt function given by

$$
f(N)=\frac{r_{0} N}{1+\frac{r_{0}-1}{K} N},
$$

where $r_{0}$ is the reproductive ratio and $K$ is the carrying capacity. Note that, in order to obtain biologically meaningful solutions, we assume that $r_{0}$ is larger than unity.

The non-dimensional form of equation (1.2.4) is given by

$$
f(N)=\frac{r_{0} N}{1+\left(r_{0}-1\right) N}
$$


which is the form we will use. The function $f$ in (1.2.5) possess the trivial equilibrium, $N^{*}=0$ and the non-trivial equilibrium $N^{*}=1$. Note that $N^{*}=0$ is unstable and $N^{*}=1$ is stable when $r_{0}$ is larger than unity.

Equation (1.2.2) with $f$ in (1.2.5) is one of the few nonlinear difference equations that has a closed solution. The substitution $u_{t}=\frac{1}{N_{t}}$ transforms (1.2.2) with $f$ in (1.2.5) into a linear difference equation which is easily solved, and the solution for $N_{t}$ is given by

$$
N_{t}=\frac{N_{0}}{N_{0}+\left(1-N_{0}\right) r_{0}^{-t}} .
$$

We observe that $N_{t}$ in (1.2.6) asymptotically approaches the nontrivial fixed point, $N^{*}=1$, as $t \rightarrow \infty$ regardless of the value of $N_{0}$ as long as $r_{0}>1$.

In a spatially explicit model such as (1.2.1), the growth function may depend on location. In this case, we write $f(N, x)$. For example, one may categorize points in space as "good" if a population can grow there and "bad" if it cannot. The scaling of the Beverton-Holt equation given by (1.2.5) requires $r_{0}>1$. Thus, on good patches, we choose equation (1.2.5) with $r_{0}=r_{0}(x)$, where it is assumed that $r_{0}$ is strictly larger than unity.

F5: $f(N ; x) \leq r_{0}(x) N$, where $r_{0}(x) \geq R_{0}>1$.

On bad patches, we choose the linearization of (1.2.5); i.e., we choose the linear function $f(N, x)=r_{0}(x) N$ with $0 \leq r_{0}<1$.

While the Beverton-Holt function is a standard choice for the growth function, the choices for dispersal kernels are less obvious and much more varied. In homogeneous landscapes, dispersal kernels have been derived from individual movement models (Neubert et al., 1995) or from data fitting (Kot et al., 1996). Regardless, dispersal in a homogeneous landscape tends to depend only on distance, so that $k(x, y)=\tilde{k}(x-y)$. By far the most commonly used models in this case are the Gaussian (1.3.14) and Laplace kernel (1.3.3), but other distributions for dispersal distances have been found empirically and used in models, e.g. Weibull kernel, a Cauchy 
distribution or a power law (Clark, 1998; Lockwood et al., 2002; Kot et al., 2004).

The choice of which dispersal kernel to use in a heterogeneous landscape is far less clear. Kawasaki and Shigesada (2007) used a Laplace kernel for a fragmented landscape. For wind-dispersed seeds, such a choice seems entirely reasonable, but when the dispersal process is affected by landscape features, new effects can occur. Dewhirst and Lutscher (2009) included heterogeneity phenomenologically by allowing the variance of a kernel to depend on initial location. More mechanistically, VanKirk and Lewis (1999) derived kernels on a single patch with a semi-permeable boundary from a random walk. Powell and Zimmermann (2004) considered a random walk in a periodic landscape and used homogenization methods to derive approximate spread rates in a patchy landscape. Robbins (2004) used almost the same model but analyzed exact persistence conditions and spread rates. Note that the choice of dispersal kernel is crucial when studying spread rates (Kot et al., 1996) and under some conditions even for persistence conditions (Lutscher et al., 2010; Zhou and Kot, 2011).

In this dissertation, we choose the mechanistic derivation of dispersal kernels as pioneered by Neubert et al. (1995) in a homogeneous landscape and generalize it to heterogeneous landscapes. This approach allows us to explicitly include movement behavior at interfaces between different landscape types by employing recent results from Ovaskainen and Cornell (2003).

\subsection{Mathematical Analysis of IDEs}

In this section, we review two important questions that may be addressed by the analysis of IDEs, along with the mathematical concepts and tools associated with each. 


\subsubsection{Critical Patch size $(\Omega$ Bounded $)$}

We consider a population residing in an infinite, one-dimensional landscape comprised of a bounded habitat patch, $\Omega$, suitable for population growth that is adjacent to matrix or uninhabitable habitat. Local population growth occurs within $\Omega$, and loss of individuals from this region is due to dispersal across the patch boundary into matrix habitat. Individuals in the matrix habitat are assumed to die immediately. Hence the growth function $f$ is the Beverton-Holt function when $x$ is in $\Omega$ and $f$ is zero outside of $\Omega$. Furthermore, it is assumed $\Omega$ is homogeneous so that $r_{0}(x) \equiv r_{0}$ and $f$ is given by (1.2.5). Without loss of generality, we take $\Omega=[-l / 2, l / 2]$ where $l$ is the length of the suitable habitat. The first question we consider is a population persistence problem. That is, when introduced at low densities, will a population eventually grow and persist? For the current setup, this corresponds to determining the critical domain size, $l^{*}$, such that the population persists precisely when $l>l^{*}$ (Skellam, 1951).

Mathematically, the question of population persistence is addressed by determining the stability properties of the zero state. We have the following definitions for local stability, attractiveness, and local asymptotic stability of $N^{*}$.

Definition 1.3.1 ( $N^{*}$ is locally stable) A fixed point, $N^{*}$, of (1.2.1) is locally stable if, for all $\epsilon>0$ there exists $\varrho>0$ such that

$$
\left\|N_{0}-N^{*}\right\|<\varrho
$$

implies that

$$
\left\|N_{t}-N^{*}\right\|<\epsilon
$$

for all $t>0$; i.e., solutions that start near $N^{*}$ stay near $N^{*}$.

Definition 1.3.2 $\left(N^{*}\right.$ is attractive) A fixed point, $N^{*}$, is attractive if there exists 
$\varrho>0$ such that

$$
\left\|N_{0}-N^{*}\right\|<\varrho
$$

implies

$$
\left\|N_{t}-N^{*}\right\| \rightarrow 0 \text { as } t \rightarrow \infty
$$

Definition 1.3.3 $\left(N^{*}\right.$ is asymptotically stable) The fixed point, $N^{*}$, is asymptotically stable if it is locally stable and attractive.

Since we excluded the Allee effect from our model, persistence conditions will be studied by considering the linearized operator at zero, namely

$$
N_{t+1}(x)=B\left[N_{t}(x)\right]:=r_{0} \int_{-l / 2}^{l / 2} k(x, y) N_{t}(y) d y
$$

where $r_{0}=f^{\prime}(0)$.

Under the additional assumption that $k$ is symmetric, i.e., $k(x, y)=k(y, x)$, $B$ is a compact (see Definition 1.3.4), linear, positive, self-adjoint operator in an appropriate function space; e.g., the space of continuous functions on $\Omega$.

Definition 1.3.4 (Compact Operator (Keener, 2000)) An operator, B, acting in a space $X$, is said to be compact if it is continuous and maps bounded sets into precompact sets. A set $S$ in $X$ is precompact if any sequence chosen from $S$ contains a subsequence that is convergent in $X$. The closure of a precompact set is compact.

From the spectral theory of compact, linear, positive self-adjoint operators, we know that a positive dominant eigenvalue corresponding to a unique (up to constant multiple), nonnegative eigenfunction exists (Lax, 2002; Zabresiko et al., 1975). Therefore, the stability of fixed points of (1.3.1) may then be studied as an eigenvalue problem

$$
\lambda \phi(x)=B[\phi(x)]
$$


Since the solution to (1.3.1) with $N_{0}(x)=C \phi(x)$ for some $C>0$ is $N_{t}(x)=C \lambda^{t} \phi(x)$, the trivial solution is locally stable when the spectral radius of $B$ is less than unity and is unstable if the spectral radius of $B$ is greater than unity.

The first application of the above theory was presented by Kot and Schaffer (1986). The authors assumed the growth function was given by the Beverton-Holt function (1.2.5) and the dispersal kernel was given by the Laplace kernel

$$
k(x, y)=\frac{a}{2} \exp (-a|x-y|)
$$

with mean dispersal distance $\frac{1}{a}$. We wish to find the critical length, $l^{*}$, that ensures survival of the population. We now derive a relation that shows how the size of the patch necessary for persistence, $l^{*}$, depends on the intrinsic growth rate, $r_{0}$, and the mean dispersal distance, $\frac{1}{a}$.

Substituting expressions (1.2.5) and (1.3.3) into (1.3.2), one obtains

$$
\lambda \phi(x)=r_{0} \frac{a}{2} \int_{-l / 2}^{l / 2} \exp (-a|x-y|) \phi(y) d y .
$$

Following Kot and Schaffer (1986), we differentiate (1.3.4) twice and obtain the following ODE for $(\lambda, \phi)$

$$
\phi^{\prime \prime}(x)+a^{2} \frac{r_{0}-\lambda}{\lambda} \phi(x)=0
$$

subject to the boundary conditions

$$
\begin{aligned}
\phi^{\prime}(l / 2)+a \phi(l / 2) & =0 \\
\phi^{\prime}(-l / 2)-a \phi(-l / 2) & =0 .
\end{aligned}
$$

Since stability of the trivial solution is lost as $\lambda$ passes through one, we set $\lambda=1$ in (1.3.5) and, applying boundary conditions (1.3.6), we obtain the following formula 


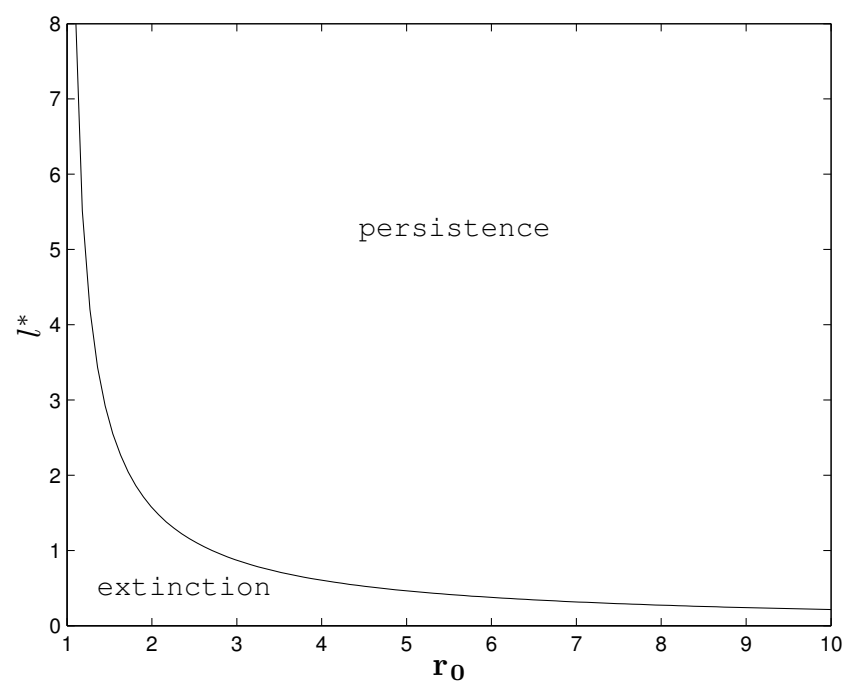

Figure 1.1: Critical patch size (equation 1.3.7) for increasing values of the intrinsic growth rate, $r_{0}$. The mean dispersal distance, $1 / a$, was set to unity.

for the critical patch size

$$
l^{*}=\frac{2}{a \sqrt{r_{0}-1}} \arctan \left(\frac{1}{\sqrt{r_{0}-1}}\right) .
$$

In Figure 1.1, we plot the critical patch size, $l^{*}$, for increasing values of $r_{0}$ and a mean dispersal distance of 1 . Observe that, as $r_{0}$ increases, the critical patch size decreases to zero. Biologically, as the number of offspring per individual per generation increases, the population can persist on a domain of decreased size. As $r_{0}$ approaches unity, observe that the critical patch size approaches infinity. Biologically, if the number of offspring per individual per generation decreases to unity, the population can never persist regardless of the patch size. This effect can also be observed from equation (1.3.7) as there are no real solutions for $l^{*}$ if $r_{0} \leq 1$.

Outside of $\Omega$ was considered completely hostile $(f=0)$ and dispersing individuals showed no behavioral response to the boundary. We will derive dispersal kernels that 
incorporate such behavior, relax the assumption of hostile exterior, and revisit the critical patch size problem in Chapter 4.

\subsubsection{Spreading Speeds and Traveling Waves ( $\Omega$ Unbounded)}

Assuming a population persists, perhaps the most important quantity to consider is the spread rate of the population and its dependence on parameters. We assume that the population resides in an infinite, one-dimensional homogeneous landscape that is suitable for population growth, thus implying $\Omega=\mathbb{R}$.

In this section, we state some results about the long-term behavior of a recursion of the form

$$
N_{t+1}(x)=\tilde{Q}\left[N_{t}\right](x):=\int_{\mathbb{R}} \tilde{k}(x-y) f\left(N_{t}(y)\right) d y
$$

In addition to the assumptions on $f$ and $k$ in Sections 1.2 and 1.3.1, the analysis in this section requires that the moment generating function of $k, M(s)$, exist for at least one positive $s$; i.e.,

$$
M(s)=\int_{-\infty}^{\infty} k(z) \exp (s z) d z<\infty
$$

The mathematical theory of spatial spread of invading organisms largely focuses on establishing the existence of a monotone traveling wave solution and the asymptotic spreading speed. The asymptotic spreading speed is a quantity such that any observer who moves faster than this speed will eventually be ahead of the spreading population and thus observe a population density of zero. Likewise, any observer who moves slower than the spreading speed will eventually observe the population to exceed some positive (constant) value. There is also an instantaneous spread rate that is defined as the distance a population moves in a single generation divided by the length of the generation. We will also make reference to a traveling wave speed, the minimal traveling wave speed, the minimal speed of a traveling profile for a lin- 
ear equation, and the asymptotic spreading speed of the linearized equation (1.3.1). These quantities measure different spread rates but are closely linked as discussed below.

Mathematically, the asymptotic spreading speed of the nonlinear (1.2.1) and linearized equation (1.3.1), monotone traveling wave solution, and minimal speed of a traveling wave are defined as follows.

Definition 1.3.5 (The Asymptotic Spreading Speed) Let $\left\{N_{t}\right\}$ be a solution of (1.3.8) and assume that the initial condition $N_{0}$ has compact support, i.e. $N_{0}(x)=0$ for $|x|>\mathcal{D}$ for some $\mathcal{D}>0$. Then the quantity $c^{*}$ is called the asymptotic spreading speed, henceforth the spreading speed, if the following two conditions are satisfied

$$
\begin{aligned}
& \text { 1. } \limsup _{t \rightarrow \infty} \max _{|x| \geq c t} N_{t}(x)=0, \forall c>c^{*}, \\
& \text { 2. } \liminf _{t \rightarrow \infty} \min _{|x| \leq c t} N_{t}(x)=\tilde{N}>0, \forall c \in\left(0, c^{*}\right),
\end{aligned}
$$

where $\tilde{N}$ is the smallest positive steady state of (1.3.8).

The spreading speed for the linearized equation is analogous to Definition 1.3.5 with the second condition replaced by

$$
\liminf _{t \rightarrow \infty} \min _{|x| \leq c t} N_{t}(x)=\hat{N}>0, \forall c \in\left(0, c^{*}\right)
$$

where $\hat{N}$ is a positive constant.

Definition 1.3.6 (Monotone Traveling Wave Solution) Assume that $f(N)$ satisfies $f(0)=0$ and $f(\tilde{N})=\tilde{N}$ for some $\tilde{N}>0$. A (rightward) monotone traveling wave solution (TWS) with wave speed $c$ is a solution of the form

$$
N_{t}(x)=N(x-c t):=N(z), z=x-c t,
$$


such that $N(z)$ is nonincreasing and satisfies (1.3.8) and the asymptotic boundary conditions

$$
\lim _{t \rightarrow-\infty} N(z)=\tilde{N}, \lim _{t \rightarrow \infty} N(z)=0 .
$$

Definition 1.3.7 (Minimal Traveling Wave Speed) A value $\hat{c}$ is called the minimum traveling wave speed if there exists a positive traveling wave solution for all $c \geq \hat{c}$.

In general, there is no simple way to calculate the value of the spreading speed. Following the classical works on the Fisher equation, one may hope to find bounds for, and sometimes the exact value of, the spreading speed in terms of a linear problem (van den Bosch et al., 1990). The analogous definitions to 1.3.6 and 1.3.7 for the traveling profile of a linear equation are as follows.

Definition 1.3.8 (Traveling Profile For A Linear Equation) A (rightward) traveling profile for linear equation (1.3.1) with profile speed $c$ is a solution of the form (1.3.12) satisfying the asymptotic condition at $t=\infty$ in Definition 1.3 .6 and

$$
\liminf _{t \rightarrow \infty} \min _{|x| \leq c t} N_{t}(x)>\hat{N}>0 .
$$

A value, $\hat{c}$, is called the minimum speed of a traveling profile for a linear equation if there exists a positive traveling profile for all $c \geq \hat{c}$.

Weinberger (1982) proved that, under the assumptions of this chapter, the minimum speed of the traveling profile for a linear equation is equal to the spreading speed of the linearized equation (1.3.1). Furthermore, he proved that the spreading speed of the nonlinear equation is equal to spreading speed of the linearized equation. This concept is known as linear determinacy (van den Bosch et al., 1990), and part of the proof follows from a comparison principle. The idea is as follows. Since 
$f(N) \leq f^{\prime}(0) N$, the same holds true for $\tilde{Q}$, and the comparison principle shows that $c^{*}$ and $\hat{c}$ are bounded above by the speed of the linearized equation. If $f$ can be bounded below by $1-\eta$ times its linearization for small $N$, the same holds true for $\tilde{Q}$. In this case, the comparison principle then shows that $c^{*}$ and $\hat{c}$ are bounded below by the linearized speed. These results are summarized in the following theorem.

Theorem 1.3.9 (Linear Determinacy) Suppose that the linearization of $Q$ at $N=$ 0 is given by a linear operator $B$ such that

1. $Q[N] \geq(1-\varrho) B[N]$ for all $N$ with $0 \leq N \leq \eta$ and all positive numbers $\varrho$;

2. $Q[N] \leq B[N]$ for all $N$ with $0 \leq N \leq N^{*}$;

Then the spreading speed is linearly determined and is given by

$$
c^{*}=\inf _{s>0}\left\{\frac{1}{s} \ln f^{\prime}(0) M(s)\right\} .
$$

Remark: If there are no constant equilibria other than 0 and $N^{*}$, Weinberger (1982) proved that the traveling wave and minimal traveling wave speed are also equal to $c^{*}$ in (1.3.13). That is, $c^{*}$ is the slowest speed for which a traveling wave solution to (1.3.8) exists.

In general, it is not possible to minimize the expression in (1.3.13) and determine $c^{*}$ analytically. However, there is one notable exception where $c^{*}$ may be determined analytically. In the case that the kernel is given by,

$$
k(x)=\frac{1}{\sigma \sqrt{2 \pi}} \exp \left(\frac{-x^{2}}{2 \sigma^{2}}\right)
$$

a Gaussian kernel with mean 0 and variance $\sigma^{2}$. The moment-generating function is $M(s)=\exp \left(\frac{\sigma^{2} s^{2}}{2}\right)$. Substituting this expression for $M(s)$ into (1.3.13), one obtains $c=\sigma \sqrt{2 \ln \left(r_{0}\right)}$. For all other kernels satisfying (1.3.9), the spreading speed must be determined numerically from (1.3.13).

To illustrate the concept of a monotone TWS, we numerically integrate (1.3.8) 
with $f$ given by (1.2.5) and $k$ by (1.3.3). Observe from Figure 1.2(a) a solution with constant shape that is translated to the right by $c$ units each generation. The latter statement can also be observed from Figure 1.2(c) since the location of the wavefront is increasing at a constant rate.

To determine an approximation of $c^{*}$ from simulations, we define the average frontal speed as the speed at which the front of the population is advancing. Since, at the front of the wave, population densities are small, we define a threshold value, $\bar{N}$, corresponding to the density in ecological experiments at which the population is no longer detectable. Let $\bar{x}=\bar{x}(t)$ denote the point in space at which $N_{t}(\bar{x}(t))=\bar{N}$ so that $\bar{x}$ denotes the spatial location of the wave front at which the population is at the threshold value. Assuming $\bar{x}(0)=0$, the average frontal speed (Kawasaki and Shigesada, 2007) is defined as

$$
\bar{c}^{*}=\lim _{t \rightarrow \infty} \frac{1}{t} \sum_{j=1}^{t}\left(\bar{x}\left(t_{j}\right)-\bar{x}\left(t_{j-1}\right)\right)=\lim _{t \rightarrow \infty} \frac{\bar{x}(t)}{t} .
$$

For the parameter values used to generate Figure $1.2, \bar{c}^{*}=0.8057$ which is in close agreement with $c^{*}=0.8431$.

If the dispersal kernel decays slower than exponential, one no longer expects a monotone TWS moving with constant speed. In what is likely the most influential publication for IDEs in ecology, Kot et al. (1996) provide an excellent discussion and analysis of what to expect for this class of dispersal kernels. An example of a such a kernel is

$$
k(x, y)=\frac{a^{2}}{4} \exp (-a \sqrt{|x-y|}) .
$$

In Figure 1.2(b), we plot the solution of (1.3.8) assuming the growth function is given by (1.2.5) and the kernel is given by (1.3.16). Observe that we obtain an accelerating wave that does not maintain its shape and moves at an increasing speed. We observe from Figure 1.2(c) that the location of the wavefront grows quadratically with time 
as opposed to the case for the Laplace kernel where it grows linearly with time.

\subsection{Outline of the Thesis}

It is clear from analysis in Section 1.3 that IDEs have many appealing features. One of the most appealing features is their ability to model long-distance dispersal and account for potential long-distance dispersal events that result in accelerating invasions (Kot et al., 1996; Lutscher, 2007). However, as pointed out by Lutscher (2007), an important feature of IDEs that has not received much attention is their ability to model short-distance dispersal with much more detail than reaction-diffusion equations. Indeed, as first shown by Neubert et al. (1995) in homogeneous landscapes, dispersal kernels can be derived from mechanistic movement models in the form of reaction-diffusion equations. In this work, we generalize their approach to patchy landscapes and show that the resulting dispersal kernel is, in general, discontinuous. IDEs equipped with these discontinuous dispersal kernels are then rigorously analyzed as outlined below.

In Chapter 2, we formulate a diffusion model for the probability density function of a random walker in a patchy landscape. We allow diffusion, settling, and mortality rates to depend on patch type. Mechanisms for individual behavior at the interface between two patches are incorporated into our model. Matching conditions at the interface for the diffusion equation are derived; they depend on the underlying mechanisms of the random walk model. We show that the dispersal kernel arises as the Green's function of a second-order differential operator and derive interface conditions for the kernel.

In Chapter 3, we derive and analyze dispersal kernels in patchy landscapes. We consider three scales of dispersal ability relative to landscape heterogeneity to illustrate the kind of dispersal kernel that results from our approach. We study the dependence of dispersal characteristics, specifically dispersal success and mean 


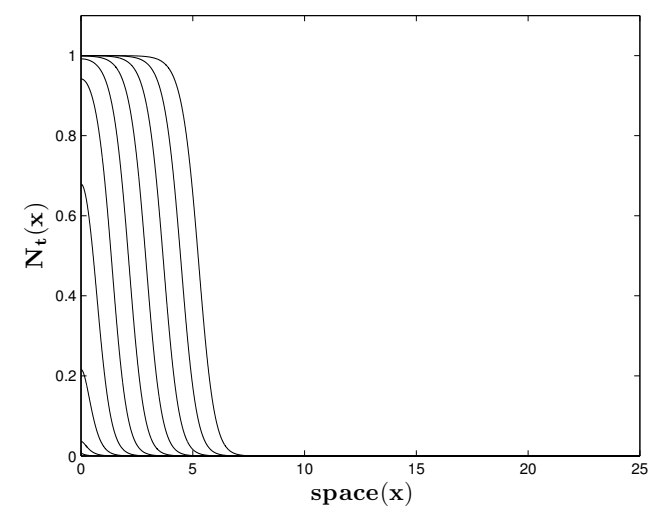

(a) Constant Profile Wave

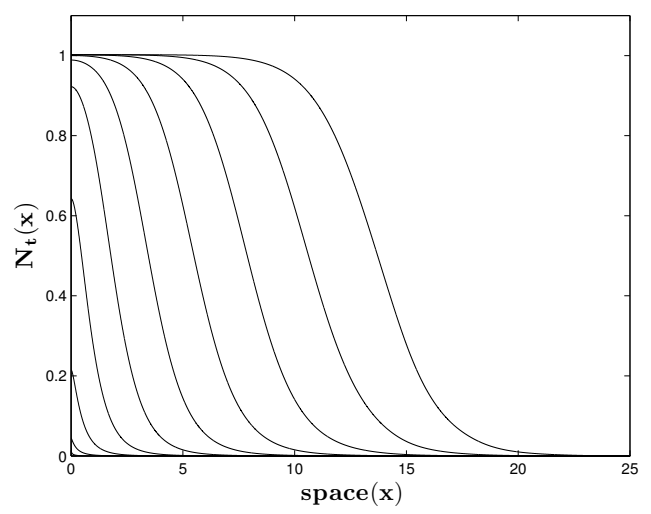

(b) Accelerating Wave

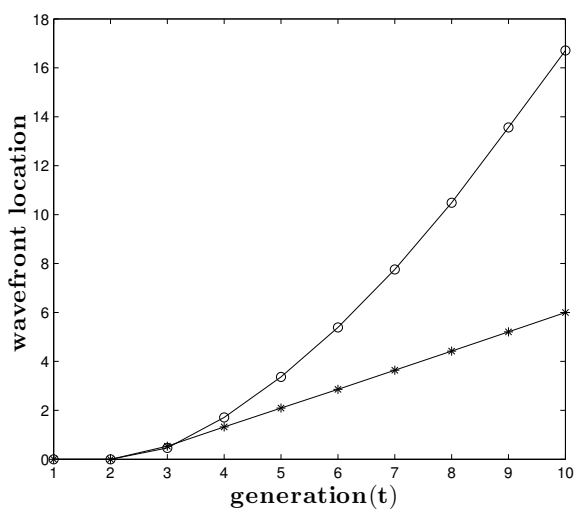

(c)

Figure 1.2: (a) Numerical solution of (1.3.8) with $f$ given by the BevertonHolt function (1.2.5) and $k$ by a Laplace kernel (1.3.3); (b) Numerical solution of (1.3.8) with $f$ given by the Beverton-Holt function (1.2.5) and $k$ by (1.3.16); (c) Location of the wavefront with $\bar{N}=1$. Parameter values are: $a=5, r_{0}=10$. 
dispersal distance, on model parameters.

In Chapter 4, we rigorously analyze an IDE in patchy landscapes. We study the critical-domain-size problem and use linearization techniques to prove the existence of a dominant eigenvalue and corresponding nonnegative eigenfunction. The dependence of this threshold on model parameters is investigated. Next, we analyze persistence conditions on an infinite, one-dimensional periodic domain. The integral operator is not necessarily compact in this case. We show that the persistence boundary is nonetheless given by an equivalent eigenvalue problem on a bounded set. We then illustrate how the interplay between dispersal ability and heterogeneity of the landscape affects persistence.

In Chapter 5, we study a traveling wave problem for an IDE in a periodic landscape. The IDE is equipped with discontinuous kernels derived in Chapters 2 and 3. Through numerical simulations, we show that the spatial profile of the invading population evolves into a discontinuous traveling periodic wave. We derive a dispersion relation for the speed of the wave, and examine the dependence of the speed on model parameters.

In Chapter 6, we develop, to the best of our knowledge, the first strategic model, sensu Pielou (1969), for the spread of EAB. Population dynamics of EAB are modeled by an IDE and host dynamics are modeled by a difference equation. Based on available data, we obtain plausible point estimates and interval ranges for model parameters. Numerical simulations show that a pulse-like solution forms as EAB invades the host population. To quantify the invasion process, we study the dependence of the speed and width of the pulse on model parameters. We employ Latin hypercube sampling and partial rank correlation coefficients to assess uncertainity in and identify important model parameters. We also investigate whether or not a barrier zone is an efficient control strategy to slow the spread of EAB. 


\section{Chapter 2}

\section{A Random Walk Model for Individual Dispersal}

Reaction-diffusion equations have been instrumental in studying spatio-temporal population dynamics ever since the seminal contributions by Fisher (1937) and Skellam (1951). As outlined in Cantrell and Cosner (2003) and Turchin (1998), reactiondiffusion equations may be derived from a detailed description of individual movement behavior in the form of a random walk model.

The work in this chapter is based on the pioneering results of Neubert et al. (1995), who considered a random walk model with constant diffusion and settling times, and derived corresponding dispersal kernels. VanKirk (1995) extended the results of Neubert et al. (1995) by showing how dispersal kernels can be characterized as the Green's function of a second-order differential operator. VanKirk and Lewis (1999) derived kernels on a single patch with a semi-permeable boundary from a random walk. Robbins (2004) considered a random walk model on a periodic landscape with constant diffusion and a patch-dependent settling time, and assumed the density was continuous across an interface.

The above models did not explore the effect of dispersal-induced mortality nor the 
possibility of movement bias at an interface of two patch types on dispersal kernels. In this chapter, we generalize the above results by considering a random-walk model on a patchy landscape with patch-dependent diffusion, settling, and mortality rates. Most importantly, we integrate recent results by Ovaskainen and Cornell (2003) on individual behavior at the interface between two patch types that includes a different mechanism for individual movement bias at an interface. In general, these interface conditions result in the probability density of the random walk being discontinuous at an interface. We derive the corresponding dispersal kernels that, in general, are also discontinuous at an interface.

This chapter is organized as follows. In Section 2.1, we formulate a random walk model in a one-dimensional, heterogeneous landscape and, under appropriate scalings, we obtain a general dispersal model for an individual in the form of a reactiondiffusion equation. In Section 2.2, we show how the dispersal kernel arises as the Green's function of a second-order, linear differential operator. In Section 2.3, the general dispersal model is formulated in a patchy landscape comprised of a single interface point. In this case, a piecewise defined system of reaction-diffusion equations arises and matching conditions for the density and its derivative are required at the interface. Depending on the underlying mechanisms of the corresponding randomwalk model, three possible interface conditions arise. In Section 2.4, the dispersal kernel is formulated in a patchy landscape and analogous interface conditions to the reaction-diffusion framework are derived for the kernel.

\subsection{The Model}

We assume that the movement of individuals follows a random walk in an infinite, one-dimensional, heterogeneous landscape. During each time step of length $\Delta t$, individuals may or may not move from their current location. The probability of dying during each time step is denoted by $\beta \Delta t$. If an individual does not succumb to 
mortality, the probability of moving from its current location is $p \Delta t$. Movement is unbiased, so that if an individual moves, the probability of moving left or right is $1 / 2$. Each move is a step of length $\Delta x$. Furthermore, the probability that an individual ends the movement process and settles at its current location is given by $\alpha \Delta t$.

Now, let $u(x, T ; y)$ denote the probability density of finding an individual at some location $x$ at some time $T$, assuming the individual emerged from a given location $y$ at time zero. In the parabolic limit, when $\Delta x, \Delta t \rightarrow 0$ in such a way that the quotient is fixed, we obtain

$$
\lim _{\Delta x, \Delta t \rightarrow 0} \frac{(\Delta x)^{2}}{2 \Delta t}:=D
$$

and the time evolution of $u(x, T ; y)$ is governed by the following partial differential equation (Turchin, 1998)

$$
\frac{\partial u}{\partial T}=\frac{\partial^{2}}{\partial x^{2}}(\nu(x) u)-(\alpha(x)+\beta(x)) u, \quad u(x, 0 ; y)=\delta(x-y)
$$

where $\nu(x):=D p(x)$ is the spatially varying motility term. By definition, the three parameter functions need to be nonnegative; in fact, we assume that $\alpha$ and $\nu$ are strictly positive and bounded away from zero. Furthermore, we assume that all three functions are bounded from above.

\subsection{The Dispersal Kernel}

According to the definition of the dispersal kernel, the quantity $k(x, y) \Delta x$ is the probability that an individual who started its dispersal process at location $y$ will settle in $[x, x+\Delta x)$ at the end of the dispersal period. According to the random walk model, the probability that an individual settles in $[x, x+\Delta x)$ during $[T, T+\Delta t)$ is $\alpha(x) u(x, T ; y) \Delta x \Delta t$. By making the biological assumption that all individuals have settled or died by the end of the dispersal period, one may assume that the dispersal 
period is infinitely long. Then, the dispersal kernel is given by

$$
k(x, y):=\int_{0}^{\infty} \alpha(x) u(x, T ; y) d T
$$

Neubert et al. (1995) used this approach for constant motility, time-dependent settling and no mortality. Since we include mortality in the dispersal process, we have

$$
\int_{-\infty}^{\infty} k(x, y) d x \leq 1
$$

and naturally

$$
\lim _{|x| \rightarrow \infty} k(x, y) \rightarrow 0, \quad \text { for all } y
$$

Equivalently, $k(x, y)$ can be constructed as the Green's function of a secondorder, linear differential operator. Integrating equation (2.1.2) from $T=0$ to $T=\infty$ and substituting the definition in (2.2.1), we obtain

$$
u(x, \infty ; y)-u(x, 0 ; y)=\int_{0}^{\infty} \frac{\partial^{2}}{\partial x^{2}}(\nu(x) u(x, T ; y)) d T-\frac{\alpha(x)+\beta(x)}{\alpha(x)} k(x, y)
$$

Since we have assumed that $\alpha$ is bounded away from zero, all individuals have either settled or died at $T=\infty$; thus $u(x, \infty ; y)=0$. Following previous authors (Neubert et al., 1995; VanKirk and Lewis, 1997; Robbins, 2004), we interchange the order of the differential and integral operators in (2.2.4) and obtain

$$
-\delta(x-y)=\frac{\partial^{2}}{\partial x^{2}}\left(\frac{\nu(x)}{\alpha(x)} k(x, y)\right)-\frac{\alpha(x)+\beta(x)}{\alpha(x)} k(x, y)
$$

Therefore, $k(x, y)$ is the Green's function of the operator

$$
\mathcal{L}:=\frac{\partial^{2}}{\partial x^{2}}\left(\frac{\nu(x)}{\alpha(x)}\right)-\frac{\alpha(x)+\beta(x)}{\alpha(x)}
$$

More specifically, we require that $k(x, y)$ is continuous in $x$ for all $y$, satisfies $\mathcal{L} k(x, y)=$ 
0 for $x \neq y$, and the matching condition

$$
\frac{\nu(y)}{\alpha(y)} \lim _{\epsilon \rightarrow 0}\left(\frac{\partial k(y+\epsilon, y)}{\partial x}-\frac{\partial k(y-\epsilon, y)}{\partial x}\right)=-1
$$

see, for example, Keener (2000).

Remark: The interchange in (2.2.4) requires that the parameter functions are sufficiently smooth and bounded so that $u$ in (2.2.1) is as well. In particular, it is required that $u$ and the parameter functions are at least of class $\mathcal{C}^{2}$.

When the coefficient functions $\alpha, \beta, \nu$ are spatially constant, equation (2.2.5) can be solved explicitly. Together with the above matching conditions at the release point, we obtain

$$
k(x, y)=\frac{\alpha}{2 \sqrt{\nu(\alpha+\beta)}} \exp \left(-\sqrt{\frac{\alpha+\beta}{\nu}}|x-y|\right) .
$$

When $\beta=0$, this kernel is the Laplace kernel, as previously derived by Neubert et al. (1995). We now turn to the case that the coefficient functions are not constant in space and, in fact, not smooth.

\subsection{Dispersal in Patchy Landscapes}

Natural landscapes are typically heterogeneous, so that all parameters in the randomwalk model depend on spatial location. In addition, individual preferences for certain landscape attributes can introduce movement bias; i.e., the probabilities of moving left and right are not equal. In this generality, movement models can be prohibitively difficult to parameterize. Landscape ecology considers environments as mosaics of different types of patches that are relatively homogeneous within and sufficiently different from their respective surroundings. In the simplest case, there are only two types of patches: good habitat and unfavorable matrix. Then the parameter functions in the random walk model assume only two different values (one in a good and one 
in a bad patch), and movement bias is represented as a patch preference that acts at the interface between two patches. We use the approach by Ovaskainen and Cornell (2003) to include patchiness and patch preference in the movement model.

When there is only one interface point, located at some $a \in \mathbb{R}$, say, we define the piecewise constant parameter function

$$
\alpha(x)=\left\{\begin{array}{l}
\alpha_{1}, x>a \\
\alpha_{2}, x<a
\end{array}\right.
$$

and similarly for $\beta$ and $\nu$. In addition, step length $\Delta x$ is patch-dependent and is given by another piecewise constant function, so that the diffusion coefficient resulting from the parabolic limit in (2.1.1) is also piecewise constant. As before, we assume that if an individual moves, the probability of moving left or right is $1 / 2$ for all $x \neq a$. At the interface, however, we allow for movement bias. The probability of moving to the left is $(1-z) / 2$ and the probability of moving right is $(1+z) / 2$, where $z \in(-1,1)$ is a measure of the degree of bias.

Within a patch, the probability density of an individual satisfies the same equation as above; i.e.,

$$
\frac{\partial u}{\partial T}(x, T ; y)=\left\{\begin{array}{l}
\nu_{1} \frac{\partial^{2} u(x, T ; y)}{\partial x^{2}}-\left(\alpha_{1}+\beta_{1}\right) u(x, T ; y), x>a \\
\nu_{2} \frac{\partial^{2} u(x, T ; y)}{\partial x^{2}}-\left(\alpha_{2}+\beta_{2}\right) u(x, T ; y), x<a
\end{array}\right.
$$

where $\nu_{i}=D_{i} p_{i}, i=1,2$ denote the motility in the two patch types. At the interface point, we require matching conditions for the density and its derivative. Depending on the assumptions on the step size $\left(\Delta x_{i}\right)$ and movement probabilities $\left(p_{i}\right)$ in the two patch types, the following different interface conditions may arise (Ovaskainen and Cornell, 2003).

Case M: Suppose that the movement probabilities in the two patch types are 
different but the step sizes are equal. Then the interface conditions become

$$
\begin{aligned}
& \nu_{2}(1+z) u\left(a^{-}, T ; y\right)=\nu_{1}(1-z) u\left(a^{+}, T ; y\right) \\
& \nu_{2} \frac{\partial u\left(a^{-}, T ; y\right)}{\partial x}=\nu_{1} \frac{\partial u\left(a^{+}, T ; y\right)}{\partial x} .
\end{aligned}
$$

The notation $a^{ \pm}$is short for the limit as $x$ approaches $a$ from above or below, respectively. The second condition in (2.3.3) represents continuity of the flux across the interface, so that no individuals are added or removed at the interface. The first condition shows that the density across an interface need not be continuous as previous authors had assumed (e.g. Shigesada et al. (1986)). The size of the discontinuity not only depends on the movement bias, $z$, but also on the fraction, $\frac{\nu_{1}}{\nu_{2}}$, of the motility coefficients in the different patches.

Case S: If one assumes that the movement probabilities are equal but the step sizes are different in the two patch types, the interface conditions are now given by

$$
\begin{aligned}
& \sqrt{\nu_{2}}(1+z) u\left(a^{-}, T ; y\right)=\sqrt{\nu_{1}}(1-z) u\left(a^{+}, T ; y\right) \\
& \nu_{2} \frac{\partial u\left(a^{-}, T ; y\right)}{\partial x}=\nu_{1} \frac{\partial u\left(a^{+}, T ; y\right)}{\partial x} .
\end{aligned}
$$

The interpretation of the conditions is the same as above, except that the discontinuity now depends on the square root of the fraction.

Case C: A number of previous authors had considered reaction-diffusion equations for random walks in patchy landscapes with the requirement that solutions be continuous across an interface (Shigesada et al., 1986). Continuity conditions for the density were derived by Nagylaki (1976) under the assumption that an individual moves from the interface into patch $i$ with the same probability as it moves left and right within patch $i$. In our notation, these assumptions read $(1-z) \nu_{1}=(1+z) \nu_{2}$. Together with the assumption of equal step sizes between the two patches, Nagylaki 
(1976) obtained the conditions

$$
\begin{aligned}
u\left(a^{-}, T ; y\right) & =u\left(a^{+}, T ; y\right) \\
\nu_{2} \frac{\partial u\left(a^{-}, T ; y\right)}{\partial x} & =\nu_{1} \frac{\partial u\left(a^{+}, T ; y\right)}{\partial x} .
\end{aligned}
$$

In particular, movement bias is not an independent parameter but rather tied to movement rates in the respective patches. We include this scenario here for comparison purposes, but we note that it cannot accommodate some desirable assumptions. For example, foraging theory suggests that an individual should move slowly in good patches and into good patches with high probability. The opposite is true for movement in and into bad patches. The condition $(1-z) \nu_{1}=(1+z) \nu_{2}$ contradicts that. For a detailed discussion on the effect of the different movement assumptions on reaction-diffusion equations, please see Maciel and Lutscher (2013).

A detailed derivation of the interface conditions (2.3.3)-(2.3.5) is given in Appendix C.

\subsection{Dispersal Kernels in Patchy Landscapes}

The definition of the dispersal kernel in (2.2.1) remains unchanged in patchy landscapes, but the characterization as a Green's function requires interface conditions analogous to (2.3.3)-(2.3.5) for the kernel. We derive these conditions here.

Case M: According to definition (2.2.1), and assuming $x>a$, one has

$$
k(x, y)=\alpha_{1} \int_{0}^{\infty} u(x, T ; y) d T
$$

Taking the limit as $x$ approaches $a$ from the right, we obtain

$$
\lim _{x \rightarrow a^{+}} k(x, y)=\alpha_{1} \lim _{x \rightarrow a^{+}} \int_{0}^{\infty} u(x, T ; y) d T .
$$


As shown in Appendix B, we can interchange the order of the limit and integral operators in (2.4.2) to obtain

$$
\lim _{x \rightarrow a^{+}} k(x, y)=\alpha_{1} \int_{0}^{\infty} \lim _{x \rightarrow a^{+}} u(x, T ; y) d T
$$

and in a similar fashion

$$
\lim _{x \rightarrow a^{-}} k(x, y)=\alpha_{2} \int_{0}^{\infty} \lim _{x \rightarrow a^{-}} u(x, T ; y) d T .
$$

Applying the first condition in (2.3.3) to equation (2.4.3), we obtain

$$
\begin{aligned}
\lim _{x \rightarrow a^{+}} k(x, y) & =\alpha_{1} \int_{0}^{\infty} \lim _{x \rightarrow a^{+}} u(x, T ; y) d T \\
& =\alpha_{1} \int_{0}^{\infty} \frac{\nu_{2}(1+z)}{\nu_{1}(1-z)} \lim _{x \rightarrow a^{-}} u(x, T ; y) d T \\
& =\frac{\alpha_{1} \nu_{2}(1+z)}{\alpha_{2} \nu_{1}(1-z)} \lim _{x \rightarrow a^{-}} k(x, y),
\end{aligned}
$$

and therefore one interface condition for $k(x, y)$ is

$$
\nu_{2} \alpha_{1}(1+z) k\left(a^{-}, y\right)=\nu_{1} \alpha_{2}(1-z) k\left(a^{+}, y\right)
$$

Similarly, we derive interface conditions for the derivatives as

$$
\alpha_{1} \nu_{2} \frac{\partial k\left(a^{-}, y\right)}{\partial x}=\alpha_{2} \nu_{1} \frac{\partial k\left(a^{+}, y\right)}{\partial x}
$$

In summary, the interface conditions for the kernel are

$$
\begin{aligned}
k\left(a^{-}, y\right) & =\bar{z} \gamma_{1} k\left(a^{+}, y\right) \\
\frac{\partial k\left(a^{-}, y\right)}{\partial x} & =\gamma_{1} \frac{\partial k\left(a^{+}, y\right)}{\partial x}
\end{aligned}
$$

where $\bar{z}=\frac{1-z}{1+z}$ and $\gamma_{1}=\frac{\nu_{1} \alpha_{2}}{\nu_{2} \alpha_{1}}$. The first condition in (2.4.8) shows that, in general (i.e. $\bar{z} \gamma_{1} \neq 1$ ), the kernel is not continuous at the interface with the size of the discontunity 
given by $\bar{z} \gamma_{1}$.

Case S: Following a similar procedure as above for Case M, one may show that the equivalent conditions to (2.3.4) for $k(x, y)$ are given by

$$
\begin{aligned}
k\left(a^{-}, y\right) & =\bar{z} \gamma_{2} k\left(a^{+}, y\right) \\
\frac{\partial k\left(a^{-}, y\right)}{\partial x} & =\gamma_{1} \frac{\partial k\left(a^{+}, y\right)}{\partial x},
\end{aligned}
$$

where $\gamma_{2}=\frac{\sqrt{\nu_{1}} \alpha_{2}}{\sqrt{\nu_{2}} \alpha_{1}}$, and $\gamma_{1}$ is as defined in (2.4.8).

Case C: The equivalent conditions to $(2.3 .5)$ for $k(x, y)$ are given by

$$
\begin{aligned}
k\left(a^{-}, y\right) & =\gamma_{3} k\left(a^{+}, y\right) \\
\frac{\partial k\left(a^{-}, y\right)}{\partial x} & =\gamma_{1} \frac{\partial k\left(a^{+}, y\right)}{\partial x},
\end{aligned}
$$

where $\gamma_{3}=\frac{\alpha_{2}}{\alpha_{1}}$. Note that the kernel may be discontinuous at an interface even if the density of the movement model is continuous. 


\section{Chapter 3}

\section{Dispersal Kernels}

This chapter is devoted to the derivation and subsequent analysis of dispersal kernels in patchy landscapes. Dispersal data have been reported in the literature for a number of organisms, and have been incorporated into IDEs by fitting data to a number of kernels (Kot et al., 1996). These data come in the form of distributions of dispersal distances and therefore assume, at least implicitly, that the landscape is essentially homogeneous.

While some empirical work has been done for movement in patchy landscapes (Chapman et al., 2007; Houtan et al., 2007), very little has been reported for invasive forest insects. Thus, in this chapter, we use the results from the previous chapter to calculate and illustrate dispersal kernels for several biologically relevant scenarios of patchy landscapes. A second objective of this chapter is to derive summary statistics of the different kernels that arise from our treatment, specifically dispersal success and mean dispersal distance. Dispersal success gives the probability that an individual successfully settles in some region and accounts for the fact that individuals may emigrate from this region or die before settling. Mean dispersal distance is a frequently reported summary statistic in empirical work on dispersal. We analyze how this quantity depends on parameters in our model. 
In Section 3.1, we assume that dispersal distance is small compared to patch size, so that a typical disperser crosses at most one interface during the dispersal phase. In Section 3.2, we consider an intermediate scale, where a single favorable, bounded patch is relatively isolated in a landscape. A typical disperser can stay in the patch or leave it. The resulting kernels will be used to study the critical patch size problem in Chapter 4. In Section 3.3, assuming that dispersal distances and landscape heterogeneity are on the same scale, we derive dispersal kernels in heterogeneous landscapes, consisting of many (periodically arranged) patches of different quality. We end with summary characteristics of dispersal as they arise from our treatment, specifically dispersal success and mean dispersal distance, in Section 3.4.

\subsection{A Single Interface}

When patches are large compared to dispersal scale, then a typical individual may encounter at most one interface during its dispersal process. We use this simple case to give examples of kernels with discontinuities and explore how the shape of these kernels depends on parameters and movement assumptions.

Without loss of generality, we choose $a=0$ as the interface between the 'good' patch (the positive $x$-axis) and the 'bad' patch (the negative $x$-axis), and $y>0$ as the release point. The Green's function for $k(x, y)$ becomes the piecewise defined system of ODEs

$$
\begin{aligned}
-\delta(x-y) & =\frac{\nu_{1}}{\alpha_{1}} \frac{\partial^{2} k(x, y)}{\partial x^{2}}-\frac{\alpha_{1}+\beta_{1}}{\alpha_{1}} k(x, y), x>0 \\
0 & =\frac{\nu_{2}}{\alpha_{2}} \frac{\partial^{2} k(x, y)}{\partial x^{2}}-\frac{\alpha_{2}+\beta_{2}}{\alpha_{2}} k(x, y), x<0,
\end{aligned}
$$

subject to interface conditions at $x=0$ and matching conditions at $x=y$. 
We solve system (3.1.1) to obtain an explicit expression for $k(x, y)$ as

$$
k(x, y)=\left\{\begin{array}{l}
A \exp \left(\mu_{2} x\right)+B \exp \left(-\mu_{2} x\right), x<0 \\
C \exp \left(\mu_{1}(y-x)\right)+D \exp \left(-\mu_{1}(y-x)\right), 0<x<y \\
E \exp \left(\mu_{1}(x-y)\right)+F \exp \left(-\mu_{1}(x-y)\right), x>y .
\end{array}\right.
$$

where $\mu_{i}=\sqrt{\frac{\alpha_{i}+\beta_{i}}{\nu_{i}}}, i=1,2$, is the inverse of the mean dispersal distance in each habitat type. Applying the asymptotic conditions (2.2.3), we require that $B=E=0$. We consider the different cases separately.

Case M: Applying (2.2.7), the continuity condition for $k$ at $x=y$, and (2.4.8) to (3.1.2), we obtain a system of linear equations for $A, C, D$ and $F$ as

$$
\begin{aligned}
& A=\bar{z} \gamma_{1}\left(C \exp \left(\mu_{1} y\right)+D \exp \left(-\mu_{1} y\right)\right) \\
& A=\frac{\mu_{1}}{\mu_{2}} \gamma_{1}\left(D \exp \left(-\mu_{1} y\right)-C \exp \left(\mu_{1} y\right)\right) \\
& C+D=F \\
& \frac{\nu_{1} \mu_{1}}{\alpha_{1}}(F+D-C)=1 .
\end{aligned}
$$

Solving (3.1.3), we obtain the dispersal kernel explicitly as

$$
k(x, y)=\left\{\begin{array}{l}
\frac{\alpha_{2}}{\nu_{2}} \hat{z}_{m} \exp \left(-\mu_{1} y+\mu_{2} x\right), x<0 \\
\chi \bar{z}_{m} \exp \left(-\mu_{1}(y+x)\right)+\chi \exp \left(-\mu_{1}(y-x)\right), 0<x<y \\
\chi\left(1+\exp \left(-2 \mu_{1} y\right) \bar{z}_{m}\right) \exp \left(-\mu_{1}(x-y)\right), x>y
\end{array}\right.
$$

where $\chi=\frac{-\alpha_{1}}{2 \sqrt{\nu_{1}\left(\alpha_{1}+\beta_{1}\right)}}$ and

$$
\hat{z}_{m}=\frac{\bar{z}}{\bar{z} \mu_{2}+\mu_{1}}, \quad \text { and } \quad \bar{z}_{m}=\frac{1-\frac{\bar{z} \mu_{2}}{\mu_{1}}}{1+\frac{\bar{z} \mu_{2}}{\mu_{1}}}
$$

represent modified bias terms. 
Figure 3.1 illustrates how the shape of the dispersal kernel is affected if only one of the parameters varies spatially. If only the death rate differs between the good and the bad patch, then the kernel is continuous. It is not symmetric, but, for the chosen parameter values, appears to be. With larger death rates (dashed curve), fewer individuals settle successfully and the kernel decays faster; see Figure 3.1(a).

The kernel is discontinuous at the interface if the motility coefficient differs from good to bad habitat; see Figure 3.1(b). When motility is lower in the good habitat, individuals spend more time there. Consequently, they are more likely to settle there even though the settling rate is the same as in bad habitat. In bad habitat, individuals move faster and, hence, spread out more. The probability density of settled individuals drops. When individuals move faster in good habitat, the situation is reversed; the likelihood of settling is low in good habitat and jumps up across the interface into bad habitat. Note that the total probability of settling (the area under the curve) does not depend on $\nu$ when $\alpha$ and $\beta$ are spatially constant. Note also that the decay rate, $\mu_{1}$, is smaller when $\nu_{1}$ is larger, so that the dashed curve is above the solid curve for large $x$. Thus the probability of long-distance dispersal in good habitat increases as $\nu_{1}$ increases.

A difference in settling rate obviously introduces a discontinuity at the interface; the side with the higher settling rate having the higher density; see Figure 3.1(c). When the settling rate is lower in good habitat, the total probability of settling is obviously lower, but again, the decay rate of the kernel is lower so that there is a (small) probability that some individuals travel (much) further.

Movement bias towards the good patch introduces a jump in the kernel at the interface as expected; see Figure 3.1(d). The overall likelihood of settling is unaffected by $z$. The size of the discontinuity in $k$ increases as the distance between the release point $y$ and the interface decreases (plot not shown). If the distance between the release point and interface is sufficiently large, movement bias has a negligible effect on $k$. 


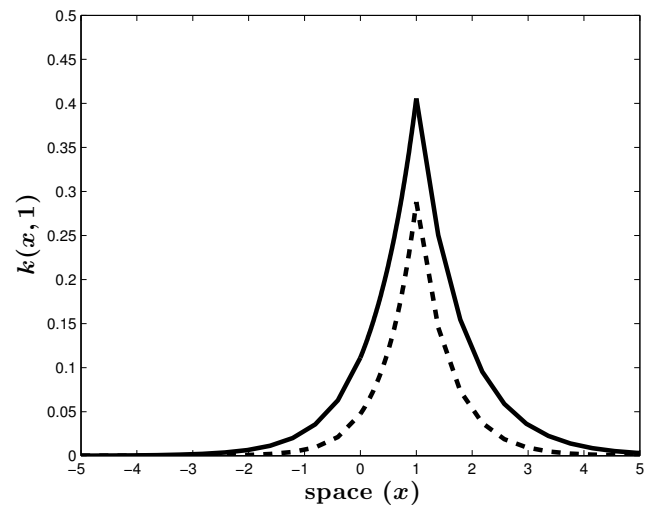

(a) mortality rates

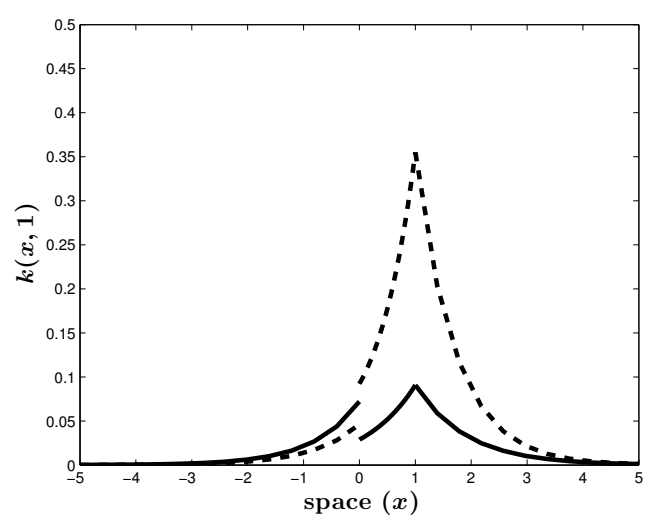

(c) settling rates

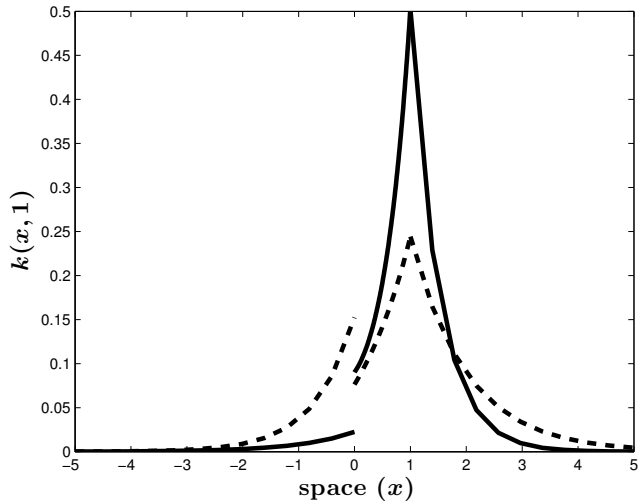

(b) motility coefficients

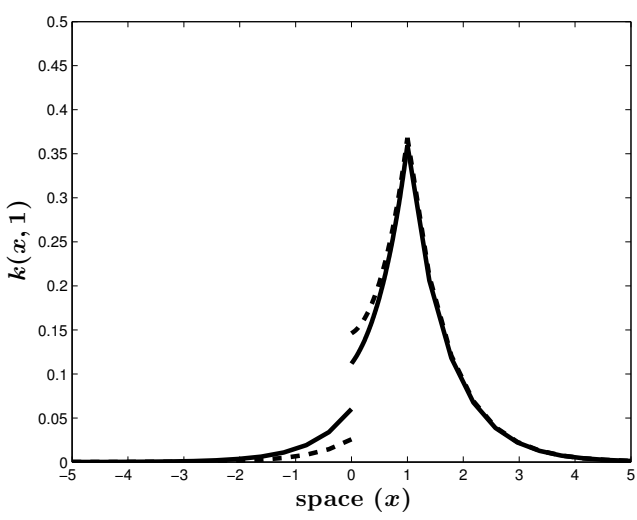

(d) movement bias

Figure 3.1: Qualitative behavior of $k(x, 1)$ assuming $x=0$ is the interface between the good patch (positive $x$-axis) and the bad patch (negative $x$ axis) for Case $\mathrm{M}$ assuming: (a) $\beta_{1}=0.5, \beta_{2}=1$ (solid) and $\beta_{1}=2, \beta_{2}=3$ (dashed), (b) $\nu_{1}=0.5, \nu_{2}=2$ (solid) and $\nu_{1}=2, \nu_{2}=1$ (dashed), (c) $\alpha_{1}=0.2, \alpha_{2}=0.5$ (solid) and $\alpha_{1}=1, \alpha_{2}=0.5$ (dashed), (d) movement bias towards the good patch given by $z=0.3$ (solid) and $z=0.7$ (dashed). In (a), (b), and (c) there was no movement bias at the interface. For all figures, all parameters not listed were set to unity. 
Following a similar procedure as for Case M above, the dispersal kernel for Case $\mathrm{S}$ is given by

$$
k(x, y)=\left\{\begin{array}{l}
\alpha_{2} \sqrt{\frac{\nu_{1}}{\nu_{2}}} \hat{z}_{s} \exp \left(-\mu_{1} y+\mu_{2} x\right), x<0 \\
\chi \bar{z}_{s} \exp \left(-\mu_{1}(y+x)\right)+\chi \exp \left(-\mu_{1}(y-x)\right), 0<x<y \\
\chi\left(1-\bar{z}_{s} \exp \left(-2 \mu_{1} y\right)\right) \exp \left(-\mu_{1}(x-y)\right), x>y,
\end{array}\right.
$$

where $\chi$ is as defined in (3.1.4), and

$$
\hat{z}_{s}=\frac{\bar{z}}{\bar{z} \sqrt{\nu_{1} \nu_{2}} \mu_{2}+\nu_{1} \mu_{1}} \quad \text { and } \quad \bar{z}_{s}=\frac{1-\frac{\nu_{1} \mu_{1}}{\bar{z} \sqrt{\nu_{1} \nu_{2}} \mu_{2}}}{1+\frac{\nu_{1} \mu_{1}}{\bar{z} \sqrt{\nu_{1} \nu_{2}} \mu_{2}}}
$$

represent modified bias terms. The dispersal kernel for Case $\mathrm{C}$ is given by

$$
k(x, y)=\left\{\begin{array}{l}
\alpha_{2} \hat{z}_{c} \exp \left(-\mu_{1} y+\mu_{2} x\right), x<0 \\
\chi \bar{z}_{c} \exp \left(-\mu_{1}(y+x)\right)+\chi \exp \left(-\mu_{1}(y-x)\right), 0<x<y \\
\chi\left(1-\bar{z}_{c} \exp \left(-2 \mu_{1} y\right)\right) \exp \left(-\mu_{1}(x-y)\right), x>y,
\end{array}\right.
$$

where $\chi$ is as defined in (3.1.4), and

$$
\hat{z}_{c}=\frac{\bar{z}}{\bar{z} \nu_{2} \mu_{2}+\nu_{1} \mu_{1}} \quad \text { and } \quad \bar{z}_{c}=\frac{1-\frac{\bar{z} \nu_{2} \mu_{2}}{\nu_{1} \mu_{1}}}{1+\frac{\bar{z} \nu_{2} \mu_{2}}{\nu_{1} \mu_{1}}}
$$

represent modified bias terms.

The qualitative features of (3.1.6) and (3.1.8) with respect to parameter functions are the same as those discussed for (3.1.4), assuming $\nu_{1}=\nu_{2}$. Differences arise when the motility coefficient in the two patch types is different (compare equations (2.4.8)(2.4.10)). The plots in Figure 3.2(a) show that, if $\nu_{1}$ is larger than $\nu_{2}$, then the fraction of individuals settling in the good patch is largest for Case C (dashed-dot) and smallest for Case $\mathrm{M}$ (dashed). If $\nu_{1}<\nu_{2}$, the fraction of individuals settling in the good patch is largest for Case M and smallest for Case C; see Figure 3.2(b). Note 


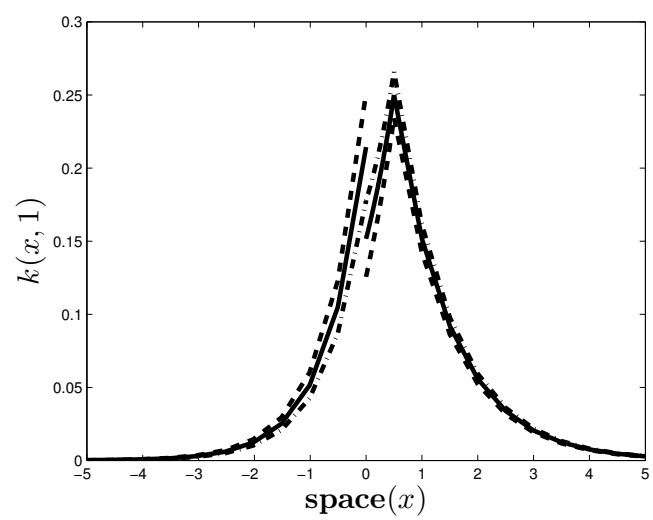

(a) $\nu_{1}$ larger

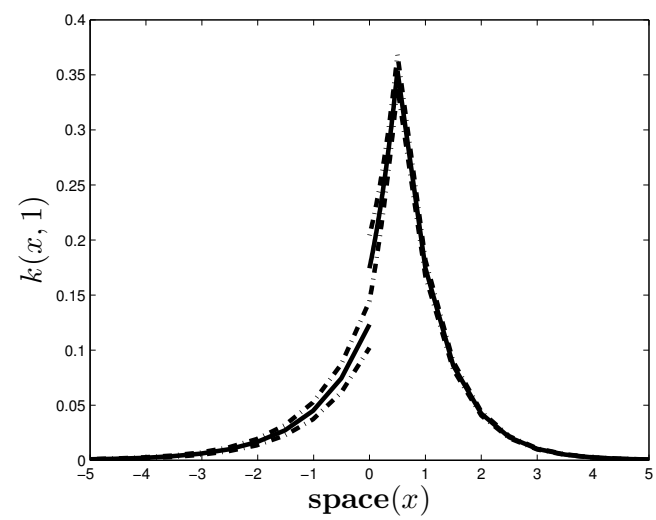

(b) $\nu_{2}$ larger

Figure 3.2: Qualitative behavior of $k(x, 1)$ assuming $x=0$ is the interface between the good patch (positive $x$-axis) and the bad patch (negative $x$-axis) for Cases $\mathrm{S}$ (solid), $\mathrm{M}$ (dashed), and $\mathrm{C}$ (dashed-dot) assuming: (a) $\nu_{1}=2$, $\nu_{2}=1$, (b) $\nu_{1}=1, \nu_{2}=2$. Fixed parameter values are: $\alpha_{1}=1, \alpha_{2}=1$, $\beta_{1}=1, \beta_{2}=1$, and $z=0$.

that the size of the discontinuity at the interface is always largest for Case M. Note also that, if $\alpha_{1}=\alpha_{2}$, then, regardless of the value of $\nu_{1}$ and $\nu_{2}$, the kernel obtained from Case $\mathrm{C}$ is continuous; see the first condition in (2.4.10). Finally, we note that, if $\alpha$ and $\beta$ are spatially constant, the total probability of settling is again independent of $\nu$.

\subsection{A Single Patch}

If dispersal occurs on the same scale as the size of a good habitat patch, and if good patches are relatively isolated, an individual can either stay inside a good patch or leave the patch and travel into unfavorable habitat. This situation is the set-up for the classical critical patch-size problem (Skellam, 1951). Here, we study how the corresponding kernels depend on parameters; the analysis of the critical patch size follows in Section 4.1. We denote the good patch of length $l_{1}$ by $\Omega=\left[-\frac{l_{1}}{2}, \frac{l_{1}}{2}\right]$. 
Parameter functions are piecewise constant; i.e.,

$$
\alpha(x)=\left\{\begin{array}{l}
\alpha_{1}, x \in \Omega \\
\alpha_{2}, x \notin \Omega,
\end{array}\right.
$$

and $\beta$ and $\nu$ are analogously defined.

The Green's function for $k(x, y)$ can now be written as three ODEs (a straightforward extension of equations (3.1.1)), coupled through appropriate interface conditions. For simplicity, we only illustrate the case that the release point occurs in the good patch. Then the dispersal kernel is given by

$$
k(x, y)=\left\{\begin{array}{l}
A_{-1} \exp \left(\mu_{2} x\right)+B_{-1} \exp \left(-\mu_{2} x\right), x<-\frac{l_{1}}{2} \\
a \exp \left(\mu_{1}(y-x)\right)+b \exp \left(-\mu_{1}(y-x)\right),-\frac{l_{1}}{2}<x<y \\
c \exp \left(\mu_{1}(x-y)\right)+d \exp \left(-\mu_{1}(x-y)\right), y<x<\frac{l_{1}}{2} \\
A_{1} \exp \left(\mu_{2} x\right)+B_{1} \exp \left(-\mu_{2} x\right), x>\frac{l_{1}}{2} .
\end{array}\right.
$$

Applying the asymptotic conditions (2.2.3), we require that $B_{-1}=A_{1}=0$. The interface conditions are given by (2.4.8), (2.4.9) or (2.4.10). In all cases, matching conditions at the release point are given by (2.2.7), and the continuity of $k$ at $x=y$.

Case M: Interface conditions (2.4.8) at $x= \pm \frac{l_{1}}{2}$, the jump condition (2.2.7) and continuity condition at $x=y$ translate into a linear system for coefficients $A_{-1}, a, b$, $c, d$, and $B_{1}$. Conveniently, these conditions can be written in matrix form $J \varphi=\kappa$ where

$$
\begin{aligned}
\varphi^{T} & =\left[A_{-1}, a, b, c, d, B_{1}\right], \\
\kappa^{T} & =\left[0,0,0,-\frac{\alpha_{1}}{\nu_{1} \mu_{1}}, 0,0\right],
\end{aligned}
$$

and

$$
J=\left(\begin{array}{cccc}
K_{1} & M_{1} & 0 & 0 \\
0 & M_{2} & M_{3} & 0 \\
0 & 0 & M_{4} & K_{2}
\end{array}\right)
$$


The entries of $J$ are matrices of the form

$$
\begin{aligned}
& K_{1}=\left(\begin{array}{c}
e^{-\mu_{2} l_{1} / 2} \\
e^{-\mu_{2} l_{1} / 2}
\end{array}\right), \quad M_{1}=\left(\begin{array}{cc}
-\bar{z} \gamma_{1} e^{\mu_{1}\left(y+l_{1} / 2\right)} & -\bar{z} \gamma_{1} e^{-\mu_{1}\left(y+l_{1} / 2\right)} \\
\frac{\mu_{1}}{\mu_{2}} \gamma_{1} e^{\mu_{1}\left(y+l_{1} / 2\right)} & -\frac{\mu_{1}}{\mu_{2}} \gamma_{1} e^{-\mu_{1}\left(y+l_{1} / 2\right)}
\end{array}\right), \\
& M_{2}=\left(\begin{array}{cc}
1 & 1 \\
1 & -1
\end{array}\right), \quad M_{3}=\left(\begin{array}{cc}
-1 & -1 \\
1 & -1
\end{array}\right) \\
& M_{4}=\left(\begin{array}{cc}
-\bar{z} \gamma_{1} e^{\mu_{1}\left(l_{1} / 2-y\right)} & -\bar{z} \gamma_{1} e^{-\mu_{1}\left(l_{1} / 2-y\right)} \\
-\frac{\mu_{1}}{\mu_{2}} \gamma_{1} e^{\mu_{1}\left(l_{1} / 2-y\right)} & \frac{\mu_{1}}{\mu_{2}} \gamma_{1} e^{-\mu_{1}\left(l_{1} / 2-y\right)}
\end{array}\right), \quad K_{2}=\left(\begin{array}{c}
e^{-\mu_{2} l_{1} / 2} \\
-e^{-\mu_{2} l_{1} / 2}
\end{array}\right),
\end{aligned}
$$

where $\bar{z}=\frac{1-z}{1+z}$, and $\gamma_{1}=\frac{\nu_{1} \alpha_{2}}{\nu_{2} \alpha_{1}}$. While the matrix equation may be solved explicitly for $\varphi$, the resulting expressions are cumbersome and do not provide significant insight into the effect of parameters on the kernel.

When the release point is in the middle of the good patch, the resulting kernels are symmetric; see Figure 3.3. Assuming that conditions are more favorable inside $\Omega$, we set $\alpha_{1}>\alpha_{2}$ and $\beta_{1}<\beta_{2}$. Then a large number of individuals settle inside the favorable patch and there is a significant drop in density across the interface when $\nu_{1}<\nu_{2}$ (solid line). When motility inside the good patch is larger than outside, the probability of settling inside the patch drops and outside increases (dashed line). In the next Section, we study how the probability of settling inside the good patch depends on parameters. When the release point is not in the middle of the good patch, the resulting kernels are asymmetric, but other characteristics are similar; see Figure 3.4(a). Movement bias at the interface can increase the probability of settling in the good patch $(z>0$, solid line, Figure 3.4(b)) or decrease it $(z<0$, dashed line, Figure 3.4(b)).

The calculations for Case S (2.4.9) and Case C (2.4.10) interface conditions are 


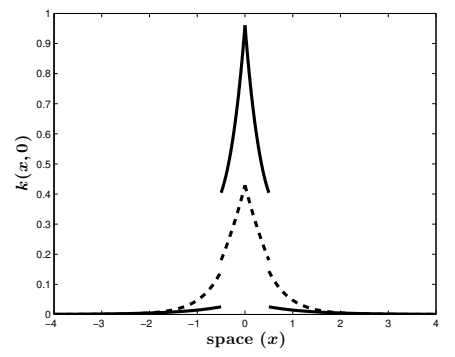

Figure 3.3: Dispersal kernels in a single bounded patch $\left(l_{1}=1\right)$ for Case M, assuming $\nu_{1}=0.5, \nu_{2}=2$ (solid) and $\nu_{1}=2, \nu_{2}=0.5$ (dashed). Fixed parameter values are: $\alpha_{1}=2.5, \alpha_{2}=0.5, \beta_{1}=0.5, \beta_{2}=2$, and $z=0$.

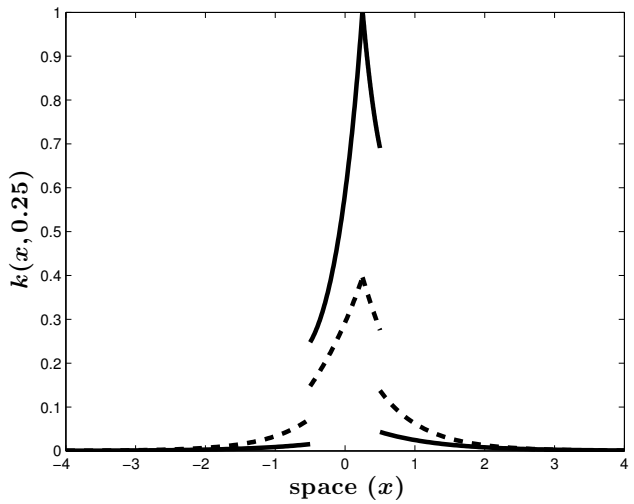

(a) no movement bias

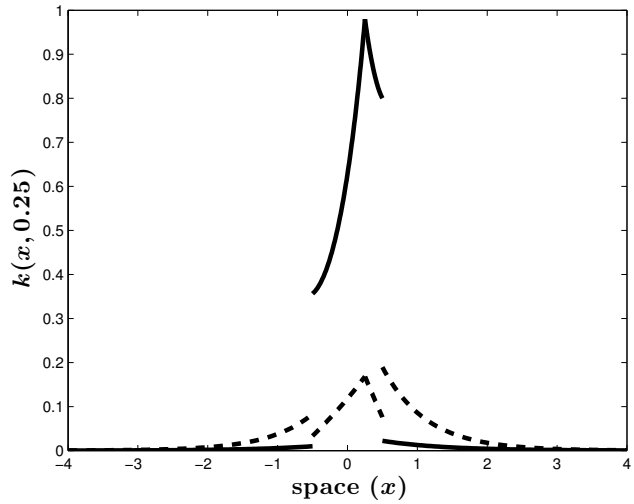

(b) movement bias

Figure 3.4: Dispersal kernels in a single bounded patch $\left(l_{1}=1\right)$ for Case $\mathrm{M}$, assuming (a) no movement bias and (b) a movement bias where $z=0.5$ (solid) and $z=-0.5$ (dashed). For both figures, $\nu_{1}=0.5, \nu_{2}=2$ (solid) and $\nu_{1}=2.5, \nu_{2}=1$ (dashed). Fixed parameter values: $\beta_{1}=0.5, \beta_{2}=2, \alpha_{1}=$ $2.5, \alpha_{2}=0.5, y=0.25$ 


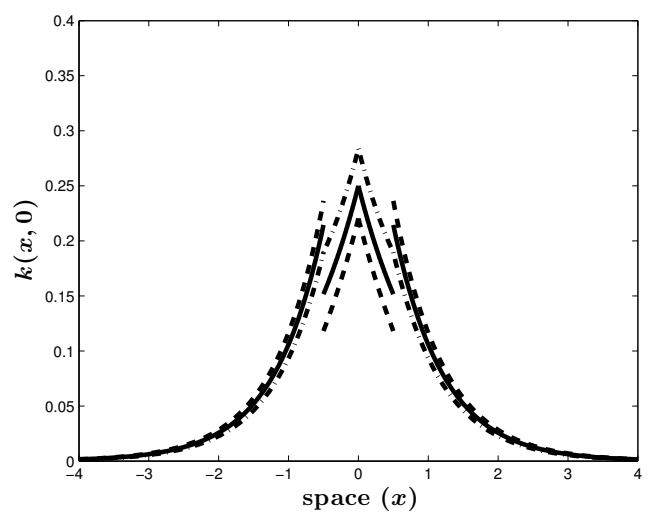

(a) $\nu_{1}$ larger

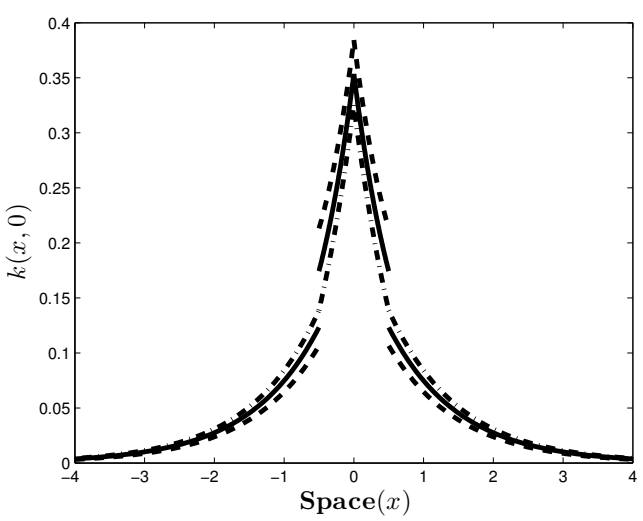

(b) $\nu_{2}$ larger

Figure 3.5: Dispersal kernels on a single isolated patch $\left(l_{1}=1\right)$ for Cases $\mathrm{S}$ (solid), M (dashed), and $\mathrm{C}$ (dashed-dot) assuming: (a) $\nu_{1}=2, \nu_{2}=1$, (b) $\nu_{1}=1, \nu_{2}=2$. Fixed parameter values are: $\alpha_{1}=1, \alpha_{2}=1, \beta_{1}=1, \beta_{2}=1$, and $z=0$.

similar to the previous case; we omit them here. The qualitative behavior with these interface conditions is similar to the previous case. The difference between Cases S, $\mathrm{M}$, and $\mathrm{C}$ depends on the ratio of the motility coefficients. When $\nu_{1}$ is larger than $\nu_{2}$, the density inside the patch is largest for Case $\mathrm{C}$ and smallest for Case M. When $\nu_{2}$ is larger than $\nu_{1}$, the situation is reversed; see Figure 3.5. Note that the size of the discontinuity at the interface is always largest for Case $\mathrm{M}$ and the kernel obtained from Case $\mathrm{C}$ is still continuous.

The kernels presented here are closely related to the kernels derived by VanKirk and Lewis (1999). Those authors considered a random walker on a single good patch with permeable boundary. Upon reaching the boundary, individuals leave the patch at a rate, $\bar{P}$, per unit length. As $\bar{P} \rightarrow 0$, dispersers tend to stay inside the patch, and they tend to leave as $\bar{P} \rightarrow \infty$. They did not model movement outside of the good 
patch, nor mortality. Their model is

$$
\begin{aligned}
& \frac{\partial u}{\partial T}=D \frac{\partial^{2} u}{\partial x^{2}}-\alpha u, \quad u(x, 0 ; y)=\delta(x-y), \quad|x|<\frac{l_{1}}{2}, \\
& \bar{P} u\left( \pm \frac{l_{1}}{2}, T ; y\right) \pm \frac{\partial u}{\partial x}\left( \pm \frac{l_{1}}{2}, T ; y\right)=0 .
\end{aligned}
$$

Their model can be obtained from ours as follows. The general dispersal model (2.1.2) for the critical patch size problem is given by

$$
\begin{array}{ll}
\frac{\partial u}{\partial T}=\nu_{1} \frac{\partial^{2} u}{\partial x^{2}}-\left(\alpha_{1}+\beta_{1}\right) u, \quad u(x, 0 ; y)=\delta(x-y), \quad|x|<\frac{l_{1}}{2}, \\
\frac{\partial u}{\partial T}=\nu_{2} \frac{\partial^{2} u}{\partial x^{2}}-\left(\alpha_{2}+\beta_{2}\right) u, \quad|x|>\frac{l_{1}}{2},
\end{array}
$$

subject to interface conditions at $x= \pm \frac{l_{1}}{2}$. Without loss of generality, we assume these are given by (2.3.3). Assuming model parameters in the bad patches are large, the PDE for $|x|>\frac{l_{1}}{2}$ may be written as

$$
\frac{\partial^{2} u}{\partial x^{2}}-\mu_{2}^{2} u=0
$$

In order to satisfy the asymptotic conditions, the solution to (3.2.7) must be

$$
u(x)=\left\{\begin{array}{l}
A \exp \left(-\mu_{2} x\right), \quad x>\frac{l_{1}}{2} \\
B \exp \left(\mu_{2} x\right), \quad x<\frac{-l_{1}}{2} .
\end{array}\right.
$$

Following Ludwig et al. (1979), we make use of (3.2.8) to reduce (3.2.6) to a bounded domain by recasting the interface conditions as a set of boundary conditions. The boundary value problem is given by

$$
\begin{aligned}
& \frac{\partial u}{\partial T}=\nu_{1} \frac{\partial^{2} u}{\partial x^{2}}-\left(\alpha_{1}+\beta_{1}\right) u, \quad u(x, 0 ; y)=\delta(x-y), \quad|x|<\frac{l_{1}}{2}, \\
& \bar{z} \mu_{2} u\left( \pm \frac{l_{1}}{2}, T ; y\right) \pm \frac{\partial u}{\partial x}\left( \pm \frac{l_{1}}{2}, T ; y\right)=0,
\end{aligned}
$$


where $\bar{z}=\frac{1+z}{1-z}$. As $z \rightarrow 1, \bar{z} \rightarrow \infty$ and individuals tend to leave the patch whereas when $z \rightarrow-1, \bar{z} \rightarrow 0$ and individuals tend to remain inside the patch.

We can identify $\bar{P}$ with $\bar{z} \mu_{2}$. In particular, we see that the rate of leaving the patch depends on the probability $z$ (through $\bar{z}$ ), and the mean dispersal distance

outside the patch, $\frac{1}{\mu_{2}}$. As individuals become more mobile outside the patch, the effective rate of entering the patch increases; see Figure 3.9(b). That is, if $\nu_{2}$ increases then $\mu_{2}$ decreases and hence $\bar{P}$ decreases, which implies a lower rate of leaving the patch.

\subsection{Several Patches}

When habitat heterogeneity and dispersal distance are on the same scale, an individual may encounter several habitat patches and corresponding interfaces during the dispersal phase. We generalize the approach from the previous section to the case of several good patches of equal size with equal-sized gaps between them; see Figure 3.6. More formally, we assume

1. The landscape consists of $2 n+1$ good patches of length $l_{1}$ with $2 n$ bad patches of length $l_{2}$ interspersed.

2. The patchy landscape is surrounded by two semi-infinite bad patches.

3. Good patches occur in $\Omega_{m}^{g}=\left(m_{-}, m_{+}\right)$where $m_{ \pm}=m l \pm \frac{l_{1}}{2}, m=-n, \ldots, n$.

4. Bad patches occur in the intervals

$$
\Omega_{m}^{b}= \begin{cases}\left((m-1)_{+}, m_{-}\right), & m>0 \\ \left(m_{+},(m-1)_{-}\right), & m<0 .\end{cases}
$$

A depiction of the patchy landscape is shown in Figure 3.6 for $n=2$. For simplicity, we will from now on assume that the release point, $y$, occurs in the center interval 


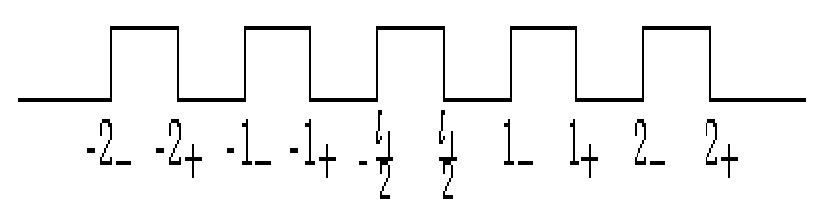

Figure 3.6: A depiction of the patchy landscape assuming $n=2$ (5 good patches). The landscape is surrounded by two semi-infinite bad patches to ensure the asymptotic conditions of $k$ are satisfied.

$\Omega_{0}^{g}$.

The dispersal kernel satisfies the following system of piecewise defined ODEs

$$
\begin{aligned}
& \delta(x-y)=\frac{\nu_{1}}{\alpha_{1}} \frac{\partial^{2} k(x, y)}{\partial x^{2}}-\frac{\alpha_{1}+\beta_{1}}{\alpha_{1}} k(x, y), \quad x \in \Omega_{0}^{g}, \\
& 0=\frac{\nu_{1}}{\alpha_{1}} \frac{\partial^{2} k(x, y)}{\partial x^{2}}-\frac{\alpha_{1}+\beta_{1}}{\alpha_{1}} k(x, y), \quad x \in \Omega_{m}^{g}, m \neq 0 \\
& 0=\frac{\nu_{2}}{\alpha_{2}} \frac{\partial^{2} k(x, y)}{\partial x^{2}}-\frac{\alpha_{2}+\beta_{2}}{\alpha_{2}} k(x, y), \quad x \in \Omega_{m}^{b} .
\end{aligned}
$$

Solving (3.3.1), we can formally write the kernel as

$$
k(x, y)=\left\{\begin{array}{l}
a e^{\mu_{1}(y-x)}+b e^{-\mu_{1}(y-x)},-l_{1} / 2<x<y \\
c e^{\mu_{1}(x-y)}+d e^{-\mu_{1}(x-y)}, y<x<l_{1} / 2 \\
C_{m} e^{\mu_{1} x}+D_{m} e^{-\mu_{1} x}, x \in \Omega_{m}^{g}, m \neq 0 \\
A_{m} e^{\mu_{2} x}+B_{m} e^{-\mu_{2} x}, x \in \Omega_{m}^{b} .
\end{array}\right.
$$

On the surrounding semi-infinite patches, the asymptotic condition (2.2.3) gives

$$
k(x, y)=\left\{\begin{array}{l}
A_{-(n+1)} e^{\mu_{2} x}, x \in\left(-\infty, n_{-}\right) \\
B_{n+1} e^{-\mu_{2} x}, x \in\left(n_{+}, \infty\right),
\end{array}\right.
$$


where $n_{ \pm}= \pm n l \pm \frac{l_{1}}{2}$. In total, there are

$$
2 \cdot 2 \cdot(\# \operatorname{good} \text { patches }-1)+\overbrace{4}^{m=0}+\overbrace{2}^{n_{ \pm}}=8 n+6
$$

constants to be determined.

At the boundary between the two patch types, we require interface conditions. We only give the formulae for Case $\mathrm{M}$ in detail. The interface conditions are given by

$$
\begin{aligned}
\lim _{x \rightarrow\left(m_{-}\right)^{+}} \nu_{1} \alpha_{2}(1-z) k(x, y) & =\lim _{x \rightarrow\left(m_{-}\right)^{-}} \nu_{2} \alpha_{1}(1+z) k(x, y) \\
\lim _{x \rightarrow\left(m_{-}\right)^{+}} \nu_{1} \alpha_{2} \frac{\partial k(x, y)}{\partial x} & =\lim _{x \rightarrow\left(m_{-}\right)^{-}} \nu_{2} \alpha_{1} \frac{\partial k(x, y)}{\partial x} \\
\lim _{x \rightarrow\left(m_{+}\right)^{+}} \nu_{2} \alpha_{1}(1+z) k(x, y) & =\lim _{x \rightarrow\left(m_{+}\right)^{-}} \nu_{1} \alpha_{2}(1-z) k(x, y) \\
\lim _{x \rightarrow\left(m_{+}\right)^{+}} \nu_{2} \alpha_{1} \frac{\partial k(x, y)}{\partial x} & =\lim _{x \rightarrow\left(m_{+}\right)^{-}} \nu_{1} \alpha_{2} \frac{\partial k(x, y)}{\partial x} .
\end{aligned}
$$

At the release point, $k(x, y)$ is continuous and satisfies $(2.2 .7)$.

The matching conditions (3.3.4) and conditions at $x=y$ translate into conditions for the coefficients $a, b, c, d, A_{m}, B_{m}, C_{m}$, and $D_{m}$. After a lot of algebra, these conditions can be written in matrix form $J_{1} \varphi_{1}=\kappa_{1}$, where

$$
\begin{aligned}
\varphi_{1}^{T} & =\left[A_{-(n+1)}, C_{-n}, D_{-n}, \ldots, A_{-1}, B_{-1}, a, b, c, d, A_{1}, B_{1}, \ldots, C_{n}, D_{n}, B_{n+1}\right] \\
\kappa_{1}^{T} & =\overbrace{0,0, \ldots, 0,0}^{4 n+3},-\frac{\alpha_{1}}{\nu_{1} \mu_{1}}, \underbrace{0, \ldots, 0}_{4 n+2}]
\end{aligned}
$$


and

$$
J_{1}=\left(\begin{array}{cccccccccccc}
k_{1} & k_{2}^{-} & 0 & 0 & \cdots & & & \cdots & 0 & 0 & 0 & 0 \\
0 & M_{6}^{+} & M_{5}^{+} & 0 & 0 & 0 & 0 & 0 & 0 & 0 & 0 & 0 \\
0 & 0 & M_{5}^{-} & M_{6}^{-} & 0 & 0 & 0 & 0 & 0 & 0 & 0 & 0 \\
\vdots & 0 & 0 & M_{6}^{+} & M_{5}^{+} & 0 & 0 & 0 & 0 & 0 & 0 & \vdots \\
& 0 & 0 & 0 & \ddots & \ddots & 0 & 0 & 0 & 0 & & \\
\vdots & 0 & 0 & 0 & 0 & J_{2} & 0 & 0 & 0 & 0 & 0 & \vdots \\
& 0 & 0 & 0 & 0 & 0 & M_{5}^{-} & M_{6}^{-} & 0 & 0 & \vdots & \\
0 & 0 & 0 & 0 & 0 & 0 & 0 & M_{6}^{+} & M_{5}^{-} & & & \\
0 & 0 & 0 & 0 & 0 & 0 & 0 & 0 & \ddots & \ddots & & \\
0 & 0 & 0 & 0 & 0 & 0 & 0 & 0 & 0 & M_{5}^{-} & M_{6}^{-} & 0 \\
0 & 0 & 0 & 0 & 0 & 0 & 0 & 0 & 0 & 0 & k_{2}^{+} & k_{3}
\end{array}\right),
$$

The matrices $M_{1}$ through $M_{4}$ in $J_{2}$ are as defined in (3.2.4), and the matrices $K_{1}^{ \pm}$are of the form

$$
K_{1}^{ \pm}=\left(\begin{array}{cc}
e^{ \pm \mu_{2} l_{1} / 2} & e^{\mp \mu_{2} l_{1} / 2} \\
e^{ \pm \mu_{2} l_{1} / 2} & -e^{\mp \mu_{2} l_{1} / 2}
\end{array}\right) .
$$

The matrices $k_{1}$ through $k_{3}$ are of the form

$$
\begin{aligned}
& k_{1}=\left(\begin{array}{l}
e^{\mu_{2} n_{-}} \\
e^{\mu_{2} n_{-}}
\end{array}\right), \\
& k_{3}=\left(\begin{array}{c}
e^{\mu_{2} n_{+}} \\
-e^{\mu_{2} n_{+}}
\end{array}\right), \\
& k_{2}^{ \pm}=\left(\begin{array}{cc}
-\bar{z} \gamma_{1} e^{\mu_{1} n_{ \pm}} & -\bar{z} \gamma_{1} e^{-\mu_{1} n_{ \pm}} \\
-\frac{\mu_{1}}{\mu_{2}} \gamma_{1} e^{\mu_{1} n_{ \pm}} & \frac{\mu_{1}}{\mu_{2}} \gamma_{1} e^{-\mu_{1} n_{ \pm}}
\end{array}\right),
\end{aligned}
$$


where $\bar{z}=\frac{1-z}{1+z}$, and $\gamma_{1}=\frac{\nu_{1} \alpha_{2}}{\nu_{2} \alpha_{1}}$, and the matrices $M_{5}^{ \pm}, M_{6}^{ \pm}$are given by

$$
M_{5}^{ \pm}=\left(\begin{array}{cc}
e^{\mu_{2} m_{ \pm}} & e^{-\mu_{2} m_{ \pm}} \\
e^{\mu_{2} m_{ \pm}} & -e^{-\mu_{2} m_{ \pm}}
\end{array}\right), \quad M_{6}^{ \pm}=\left(\begin{array}{cc}
-\bar{z} \gamma_{1} e^{\mu_{1} m_{ \pm}} & -\bar{z} \gamma_{1} e^{-\mu_{1} m_{ \pm}} \\
-\frac{\mu_{1}}{\mu_{2}} \gamma_{1} e^{\mu_{1} m_{ \pm}} & \frac{\mu_{1}}{\mu_{2}} \gamma_{1} e^{-\mu_{1} m_{ \pm}}
\end{array}\right), \quad m \neq 0 .
$$

We illustrate some of the resulting kernels in the case of five good patches $(n=2)$ in Figure 3.7. The most significant differences between Cases M, S, and C occur when motility varies between the two patch types. The first two panels compare the kernels for the three different interface conditions when $\nu_{1}>\nu_{2}$ (Figure 3.7(a)) and $\nu_{1}<\nu_{2}$ (Figure 3.7(b)). We observe that Case $\mathrm{C}$ has the highest density near the release site when $\nu_{1}$ is larger and the smallest when $\nu_{2}$ is larger. Concentration of settled individuals near the release point is particularly strong when $\nu_{1}<\nu_{2}$ and Cases $\mathrm{M}$ or $\mathrm{S}$ are chosen; the density drops significantly when going from the good to the nearest bad patch and increases again when entering the subsequent good patch. This alternating pattern of density decreasing (increasing) when going from one good (bad) patch into the nearest bad (good) patch is even more pronounced when individuals have a preference for good patches in Cases S and M; see Figures 3.7(c), 3.7(d).

\subsection{Dispersal Characteristics in Patchy Landscapes}

In this section, we consider two biologically relevant statistics that may be derived from the dispersal kernel, namely the dispersal success function and the mean dispersal distance. These quantities provide useful measures for retention and dispersal in patchy landscapes. Empirically, these measures are attractive since they correspond to data obtained from point-release experiments (Fagan and Lutscher, 2006). We introduce and discuss both measures in a homogeneous landscape first.

VanKirk (1995) defined the dispersal success function, $\hat{s}_{\Omega}(y)$, as the probability that an individual initially located at $y$ settles successfully within a certain domain 


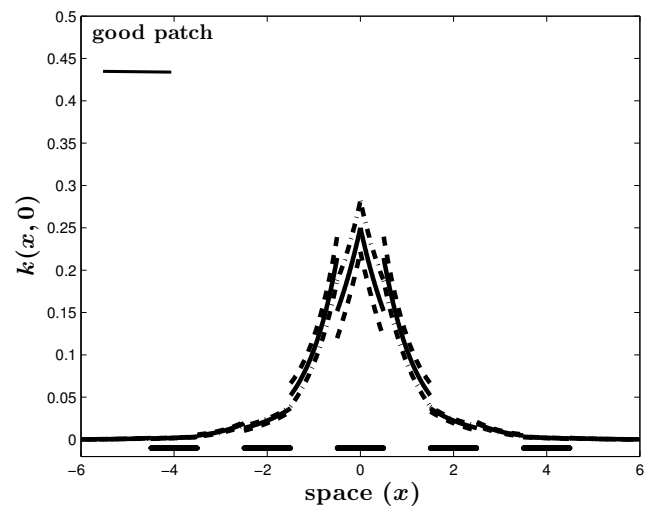

(a) $\nu_{1}$ larger

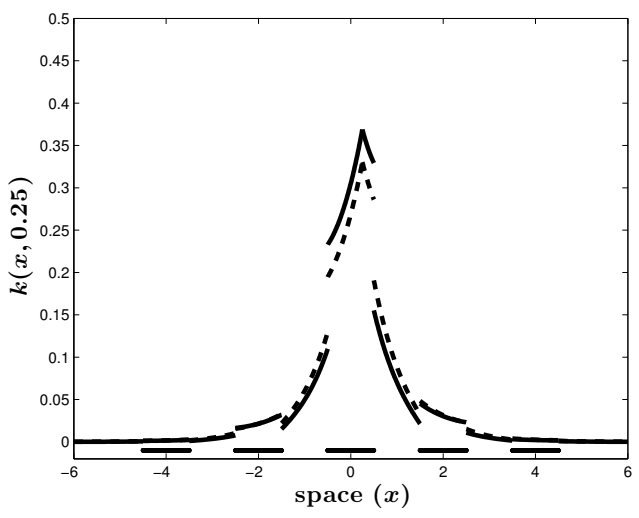

(c) $\nu_{1}$ larger; movement bias

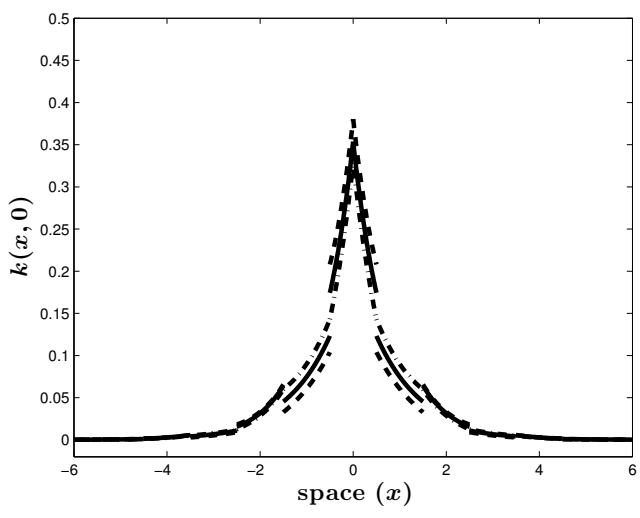

(b) $\nu_{2}$ larger

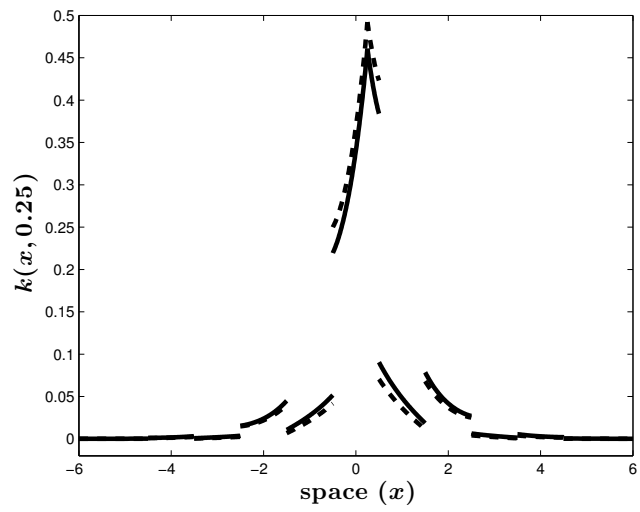

(d) $\nu_{2}$ larger; movement bias

Figure 3.7: Dispersal kernels for patchy landscape $(n=2$ or five good patches) as a function of motility and movement bias. Panel (a) corresponds to dispersal kernels obtained from Cases S (solid), M (dashed) and C (dasheddot) assuming $\nu_{1}=2, \nu_{2}=1, y=0, z=0$; Panel (b) corresponds to dispersal kernels obtained from Cases $\mathrm{S}$ (solid), $\mathrm{M}$ (dashed) and $\mathrm{C}$ (dashed-dot) assuming $\nu_{1}=1, \nu_{2}=2, y=0, z=0$; Panel (c) corresponds to dispersal kernels obtained from Cases $\mathrm{S}$ (solid) and $\mathrm{M}$ (dashed) assuming $\nu_{1}=2, \nu_{2}=1$, $y=0.25, z=0.5$; Panel (d) corresponds to dispersal kernels obtained from Cases $\mathrm{S}$ (solid) and $\mathrm{M}$ (dashed) assuming $\nu_{1}=1, \nu_{2}=2, y=0.25, z=0.5$. Fixed parameter values are: $l_{1}=1, l_{2}=1, \alpha_{1}=1, \alpha_{2}=1, \beta_{1}=1$, and $\beta_{2}=1$. 
of interest, $\Omega$. Mathematically, this function is given by

$$
\hat{s}_{\Omega}(y)=\int_{\Omega} k(x, y) d x
$$

We omit the subscript if there is no ambiguity.

In the homogeneous landscape, the dispersal success is independent of initial location. We calculate

$$
\hat{s}(y)=\hat{s}(0)=\int_{-\infty}^{\infty} \frac{\alpha}{2 \sqrt{\nu(\alpha+\beta)}} \exp \left(-\sqrt{\frac{\alpha+\beta}{\nu}}|x|\right) d x=\frac{\alpha}{\alpha+\beta} .
$$

This quantity is simply the probability that an individual settles rather than dies during the dispersal period. In general, this quantity is the scaling factor to normalize the kernel; i.e., the density of successful dispersers. Specifically, if $k(x, y)$ is some kernel obtained from (2.1.2) and (2.2.1), then the normalized kernel $\hat{k}(x, y)=k(x, y) / \hat{s}(y)$ is a probability density function for each $y$. In case of kernel (2.2.8), we obtain

$$
\hat{k}(x, y)=\frac{1}{2} \sqrt{\frac{\alpha+\beta}{\nu}} \exp \left(-\sqrt{\frac{\alpha+\beta}{\nu}}|x-y|\right) .
$$

This kernel is symmetric and has mean zero. The mean directional displacement or mean dispersal distance from a disperser's point of origin is

$$
\frac{1}{\mu}:=\int_{-\infty}^{\infty}|x| \hat{k}(x, y) d x=\sqrt{\frac{\nu}{\alpha+\beta}} .
$$

Hence, this quantity is monotonically increasing in $\nu$ and monotonically decreasing in $\alpha$ and $\beta$. 


\subsubsection{Dispersal Success in Patchy Landscapes}

The dispersal success function on a bounded patch and its mean, the average dispersal success, provide good approximations to the persistence condition of a population described by IDE (1.2.1) on a single patch and to the spatial profile of a positive steady state (VanKirk and Lewis, 1997; Lutscher and Lewis, 2004).

The dispersal success function on a single patch is concave down; it is high near the center of the domain from where individuals are likely to settle within the domain and lower near the boundary of the domain from where individuals are likely to leave the domain during dispersal. Foraging theory suggests that individuals should move more slowly in good than in bad habitat. Under this assumption, we find that dispersal success is highest in Case $\mathrm{M}$ and lowest in Case S, independent of movement bias; see Figures 3.8(a) and 3.8(b). In the case of no movement bias, dispersal success is largest for Case M and smallest for Case C; see Figure 3.8(c). In each of the cases, dispersal success is monotone increasing with patch length; see Figure 3.8(d). When the patch length is large relative to dispersal distances, the dispersal success function asymptotically approaches the fraction of dispersers successfully settling in the good patch, given by $\frac{\alpha_{1}}{\alpha_{1}+\beta_{1}}$, independent of movement assumptions.

The average dispersal success gives the spatially averaged probability, over all possible initial points $y \in \Omega$, that an individual has settled in $\Omega$ at the end of the dispersal period. Mathematically, the average dispersal success is defined as

$$
S:=\frac{1}{|\Omega|} \int_{\Omega} \hat{s}(y) d y .
$$

The product $f^{\prime}(0) S$ closely approximates the dominant eigenvalue of the linearized integral operator in (1.3.1) (Lutscher and Lewis, 2004; Fagan and Lutscher, 2006), and hence gives an approximate persistence condition.

Average dispersal success increases with bias towards the good patch and with patch size irrespective of movement assumptions; see Figures 3.9(a) and 3.9(b). When 


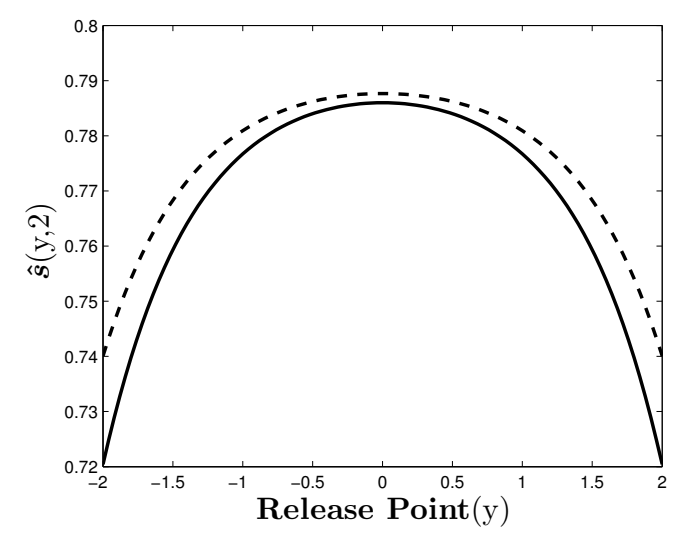

(a) movement bias towards $\Omega$

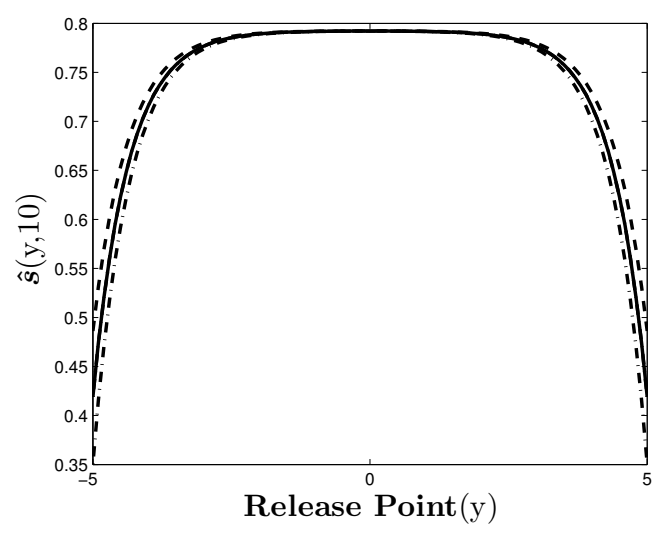

(c) no movement bias; large $l_{1}$

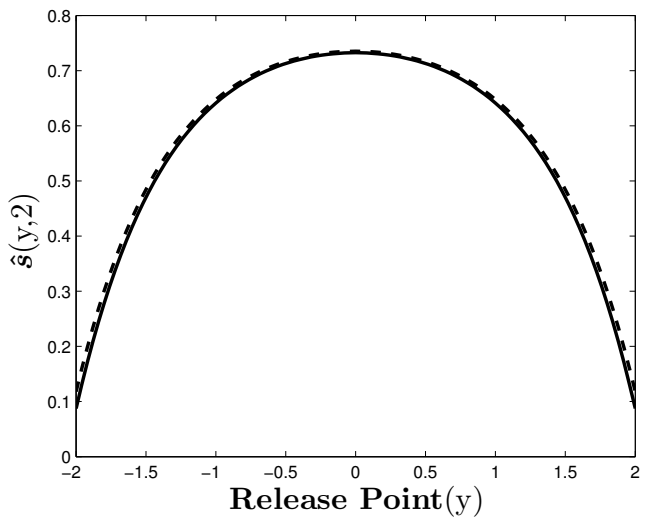

(b) movement bias away from $\Omega$

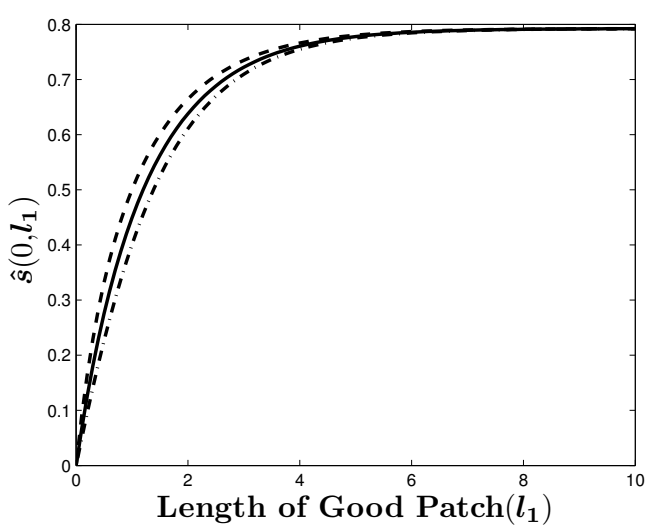

(d) no movement bias

Figure 3.8: Dispersal success function in a single patch $\Omega=\left[-\frac{l_{1}}{2}, \frac{l_{1}}{2}\right]$. Panel (a) corresponds to Cases $\mathrm{S}$ (solid) and $\mathrm{M}$ (dashed) assuming $l_{1}=2, z=0.8$; Panel (b) corresponds to Cases $\mathrm{S}$ (solid) and $\mathrm{M}$ (dashed) assuming $l_{1}=2$, $z=-0.8$; Panel (c) corresponds to Cases S (solid), M (dashed), and C (dashed-dot) assuming $l_{1}=10, z=0$; Panel (d) corresponds to Cases $\mathrm{S}$ (solid), $\mathrm{M}$ (dashed), and $\mathrm{C}$ (dashed-dot) assuming $y=0, z=0$. Fixed parameter values are: $\nu_{1}=1, \nu_{2}=2, \alpha_{1}=2, \alpha_{2}=1, \beta_{1}=0.5$, and $\beta_{2}=1$. 
no individuals leave the domain $(z=1)$ or no individuals enter the domain $(z=-1)$, the average dispersal success is independent of parameters outside the patch. When $\nu_{1}>\nu_{2}$, average dispersal success is larger for Case $\mathrm{S}$ and the situation is reversed if $\nu_{1}<\nu_{2}$, independent of movement bias; see Figures 3.9(a) and 3.9(b).

For intermediate cases, average dispersal success increases as motility outside the good patch increases (Figures 3.9(c) - 3.9(d)). Note that, the average dispersal success for Case $\mathrm{S}$ is independent of increasing values of $\nu_{2}$. This is in agreement with our result that shows the critical domain size formula for Case $\mathrm{S}$ is independent of $\nu_{2}$ (see formula (4.1.18)).

The average dispersal success on a patchy landscape with five patches is shown in Figure 3.10 for Cases M and S. As the preference for good patches increases $(z \rightarrow 1)$, there is a large reduction in the net displacement of individuals. As preference for leaving good patches increases $(z \rightarrow-1)$, the average dispersal success decreases significantly. In this case, individuals disperse a longer distance but less are successful in settling before dying. When individuals tend to settle at a higher rate in good patches and the hostility of bad patches has increased, average dispersal success increases as preference for good patches increases (dashed curve). Note that, if $z=$ \pm 1 , then the average dispersal success is independent of the movement assumptions, and for intermediate values of the bias, average dispersal success is largest for Case M.

The above results agree with a recent study by Chapman et al. (2007) who analyzed the affect of permeable boundaries on the dispersal behavior of tansy leaf beetle, Chrysolina graminis. The results of their field experiments and simulation model showed that permeability had a significant effect on net displacement of the beetle and on the inter-patch movement rate. A low permeability resulted in a low net displacement and inter-patch movement, and vice-versa as permeability increases. 


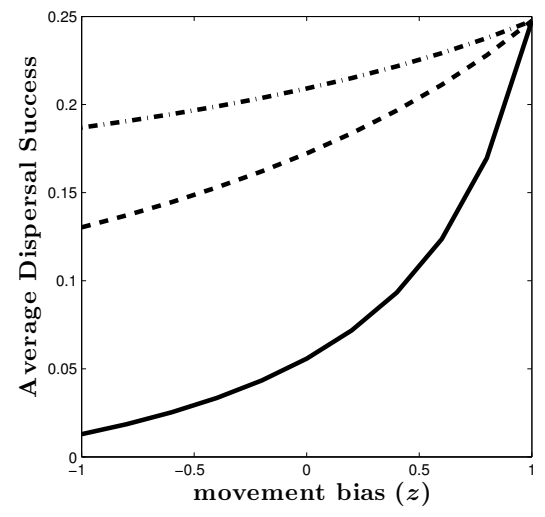

(a) $\nu_{1}, \nu_{2}$ fixed (Case M)

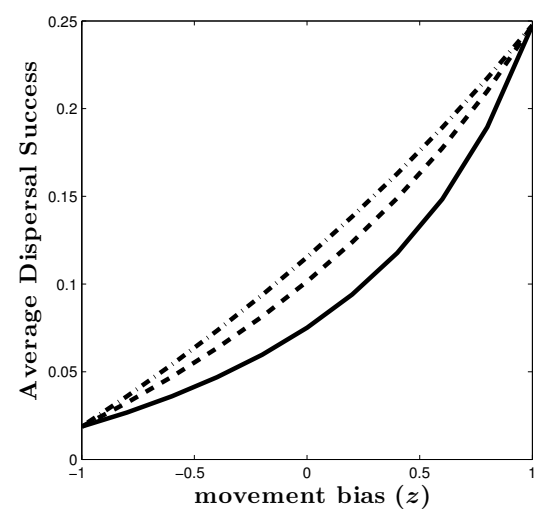

(c) $\nu_{2}$ increasing (Case $\mathrm{M}$ )

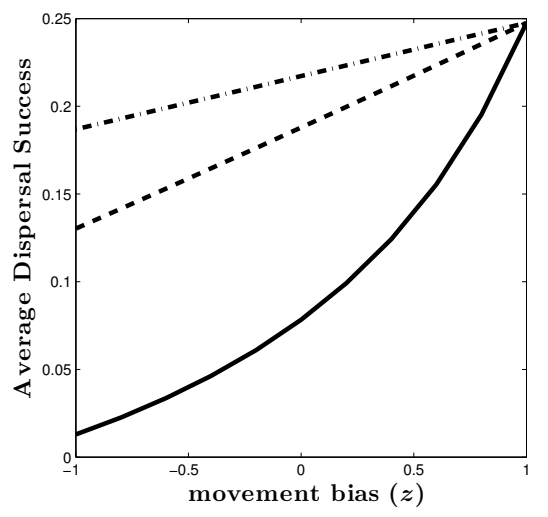

(b) $\nu_{1}, \nu_{2}$ fixed (Case $\left.\mathrm{S}\right)$

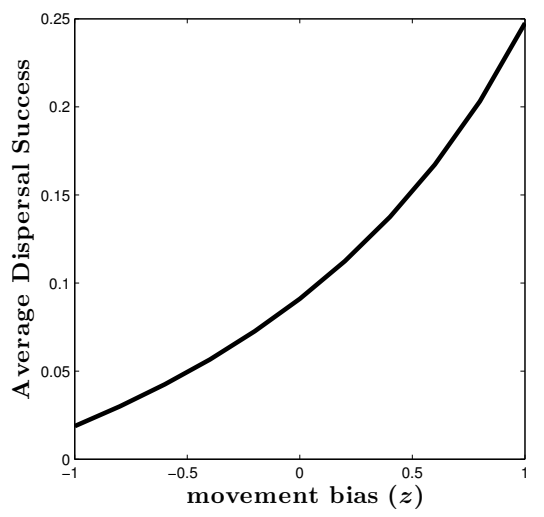

(d) $\nu_{2}$ increasing (Case S)

Figure 3.9: Average dispersal success versus movement bias in a single patch. Panel (a) corresponds to Case $\mathrm{M}$ and Panel (b) Case $\mathrm{S}$ assuming $l_{1}=1$ (solid), $l_{1}=5$ (dashed), and $l_{1}=10$ (dashed-dot), $\nu_{1}=3, \nu_{2}=1$; Panel (c) corresponds to Case $\mathrm{M}$ and (d) Case S assuming $l_{1}=1, \nu_{1}=3, \nu_{2}=1$ (solid), $\nu_{2}=3$ (dashed), $\nu_{2}=6$ (dashed-dot). Fixed parameter values are: $\alpha_{1}=1, \alpha_{2}=1, \beta_{1}=1$, and $\beta_{2}=1$. 


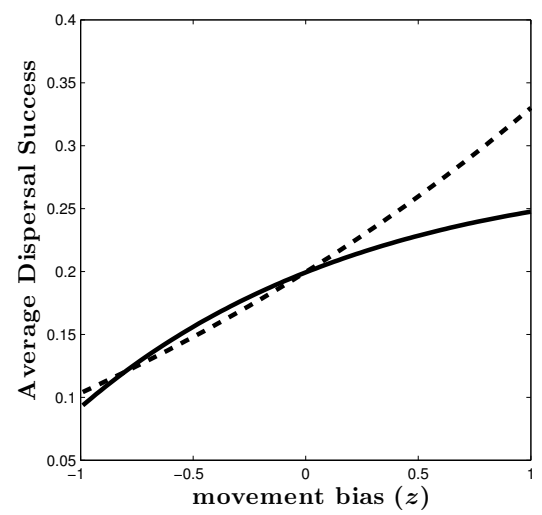

(a) Case M

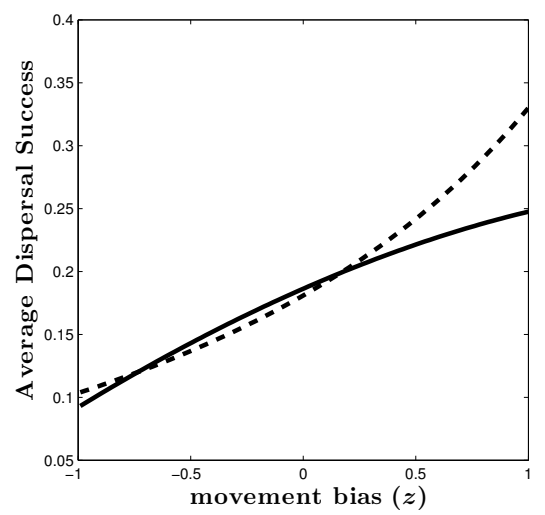

(b) Case $\mathrm{S}$

Figure 3.10: Average dispersal success versus movement bias in a patchy landscape with five patches for Case M (Panel (a)) and Case S (Panel (b)) assuming $\alpha_{1}=1, \alpha_{2}=1, \beta_{1}=1, \beta_{2}=1$ (solid) and $\alpha_{1}=1, \alpha_{2}=0.3$, $\beta_{1}=0.5, \beta_{2}=5$ (dashed). Fixed parameter values are: $\nu_{1}=1, \nu_{2}=2$, $l_{1}=2$, and $l_{2}=2$.

\subsubsection{Mean dispersal distance in a patchy landscape}

Empiricists measure dispersal distances in patchy landscapes and then give best fits for normal, negative exponential, power laws, or other functions (Chapman et al., 2007; Houtan et al., 2007). We now derive mean dispersal distances in patchy landscapes from our random-walk-modeling approach and compare the actual (discontinuous) kernels with simple distributions fitted to this mean. For simplicity, we only use five good patches $(n=2)$, and we restrict other parameters such that the mean dispersal distance does not exceed the spatial extent of these five patches. We only consider the case where $y=0$; i.e., the release point is in the middle of $\Omega_{0}^{g}$.

The panels in Figure 3.11 show that the mean dispersal distance increases for increasing values of $\nu_{1}$, as expected. Increased risk of death during dispersal significantly reduces dispersal distances (solid line). Obtained mean dispersal distances are

similar for Cases M and S, but significantly lower for Case C. Note that increasing values of $\nu_{1}$ for Case $\mathrm{C}$ also corresponds to a higher probability that an individual 
does not leave a good patch, resulting in a lower mean dispersal distance.

Mean dispersal distance is a hump-shaped function of preference for good patches, regardless of movement assumptions; see Figure 3.12. Increasing preference for good patches helps individuals survive, so that the mean dispersal distance can increase. At the same time, it decreases the likelihood that an individual will cross the gap to the next good patch, so that dispersal distance decreases. The first mechanism is stronger at low values of $z$, the second at high values. The maximum of the mean dispersal distance with respect to $z$ depends on other model parameters, particularly on mortality.

We now visually compare the kernels obtained from (3.3.2) with a Laplace kernel of equal mean dispersal distance $\frac{1}{a}$ given by equation (1.3.3).

The plots in Figure 3.13 show that, under some conditions, the two can be virtually identical, whereas in other situations, the difference can be large. Several factors affect the difference. We illustrate this for Case $\mathrm{M}$ and note that the results also hold for Case $\mathrm{S}$ and Case $\mathrm{C}$ as long as $\alpha_{1} \neq \alpha_{2}$. Low settling rates in bad patches obviously lead to very few individuals settling there, whereas the Laplace kernel (1.3.3) does not distinguish patches and overestimates the probability of settling in bad patches. Another crucial quantity is the relation of the scale of dispersal to the release patch. If the scale of dispersal is relatively small, then most individuals settle in their patch of origin (see the previous discussion on average dispersal success) and few individuals settle in other good patches. If the scale of dispersal is relatively large, then differences between the true (discontinuous) kernel (3.3.2), plotted as dashed line, and the simple (continuous) kernel (1.3.3), plotted as solid line, extends to other patches. We investigate how population persistence and spread in a patchy landscape depends on these relative scales in Section 4.4.1. 


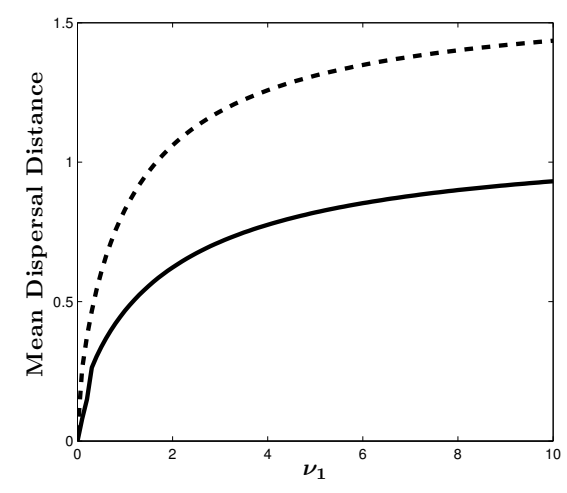

(a) Case M

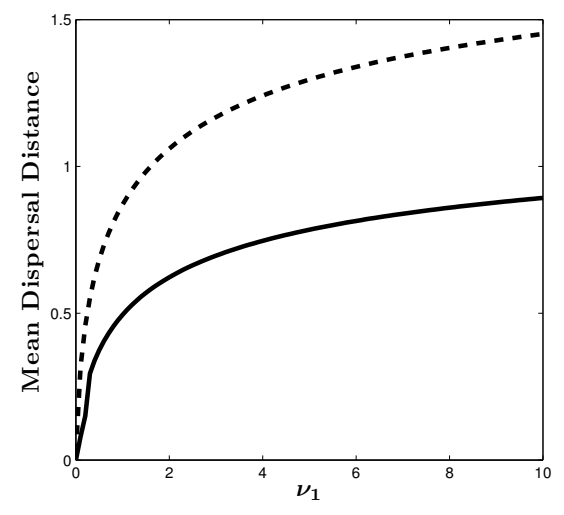

(b) Case $\mathrm{S}$

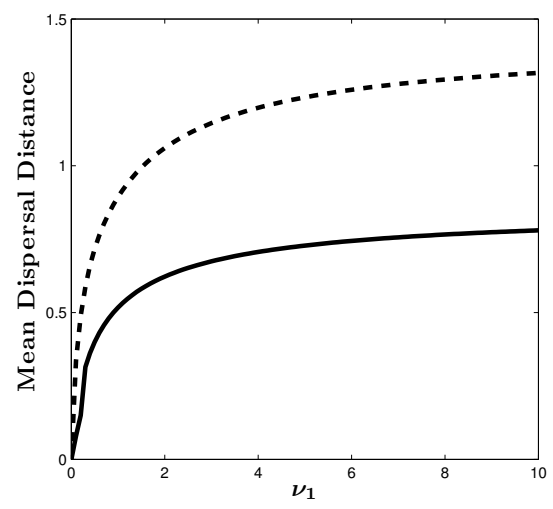

(c) Case $\mathrm{C}$

Figure 3.11: Mean dispersal distance in a patchy landscape with five good patches for Cases M, S, and $\mathrm{C}$ assuming: $\beta_{1}=0.5, \beta_{2}=1$ (dashed) and $\beta_{1}=3, \beta_{2}=5$ (solid). Fixed parameter values are: $\alpha_{1}=1, \alpha_{2}=1, \nu_{2}=2$, $l_{1}=1, l_{2}=1$, and $z=0$. 


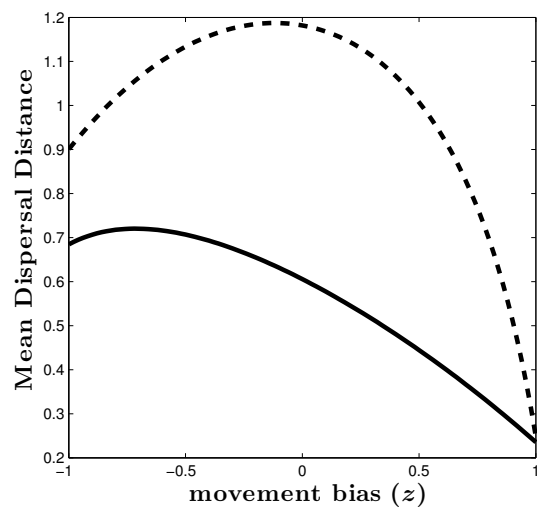

(a) movement bias; Case M

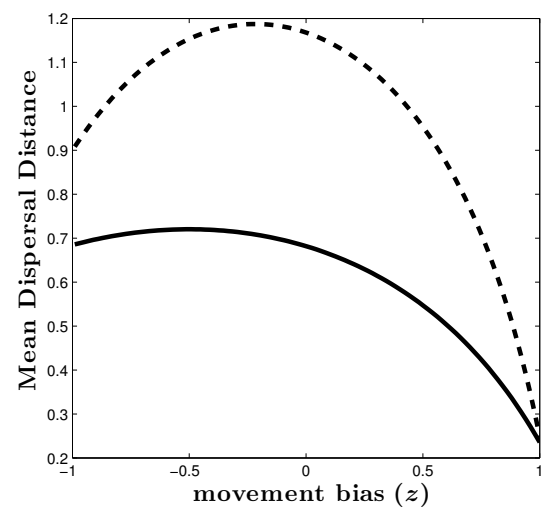

(b) movement bias; Case S

Figure 3.12: Mean dispersal distance in a patchy landscape with five good patches for Cases M and S assuming: $\nu_{1}=0.5$ (solid), $\nu_{1}=3$ (dashed), $\beta_{1}=0.5, \beta_{2}=1$. Fixed parameter values are: $\alpha_{1}=1, \alpha_{2}=1, \nu_{2}=2, l_{1}=1$, and $l_{2}=1$.

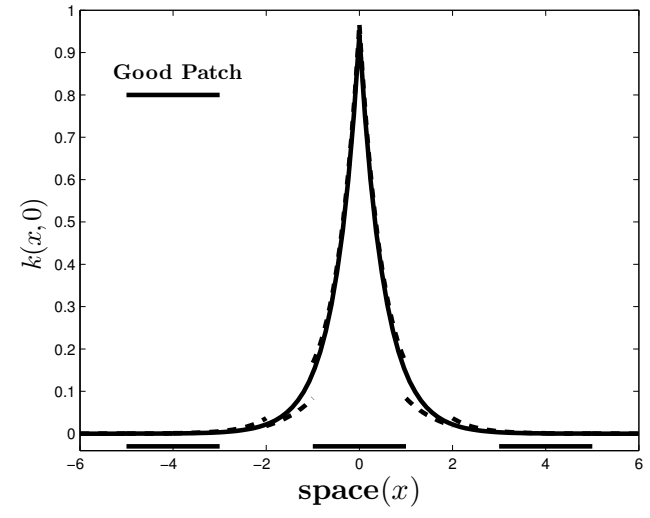

(a) large $l_{1}$

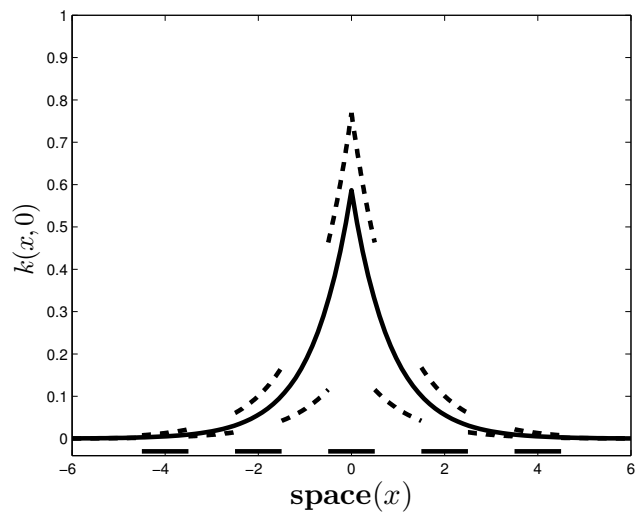

(b) small $l_{1}$

Figure 3.13: A comparison of the Laplace kernel (1.3.3) to (3.3.2) for Case $\mathrm{M}$ with equal mean dispersal distances. In (a) we set $l_{1}=2, l_{2}=1, \alpha_{1}=1$, $\alpha_{2}=1, \beta_{1}=1, \beta_{2}=1, \nu_{1}=0.5, \nu_{2}=1$, and $z=0$, (b) $l_{1}=1, l_{2}=1$, $\alpha_{1}=1.5, \alpha_{2}=0.5, \beta_{1}=0.1, \beta_{2}=1.5, \nu_{1}=1.5, \nu_{2}=2$, and $z=0$. 


\section{Chapter 4}

\section{Analysis of Integrodifference Equations: Population Persistence}

This chapter is devoted to the analysis of integrodifference equations in patchy landscapes. Specifically, we consider the recursion

$$
N_{t+1}(x)=Q\left[N_{t}\right](x):=\int_{\mathbb{R}} k(x, y) f\left(N_{t}(y) ; y\right) d y .
$$

We analyze (4.0.1) for the following two ecological situations. First, we assume the population resides in a patch of habitat suitable for population growth that is surrounded by matrix habitat unsuitable for population growth. We then consider a periodic landscape comprised of patches suitable and unsuitable for population growth. We choose to study IDEs in patchy landscapes for several reasons. As discussed in Chapter 2, models in continuously varying landscapes are prohibitively difficult to parameterize, and landscape ecologists typically consider patchy environments. Numerous studies have also found that population persistence in patchy landscapes is highly sensitive to the scale of dispersal relative to landscape heterogeneity (VanKirk, 1995; Fahrig, 2002; With, 2002; Dewhirst and Lutscher, 2009). Most importantly, we wish to consider the effect of individual movement behavior at the interface of two 
patch types on persistence. In this chapter, we provide a framework for analyzing the effect of different mechanisms at an interface on persistence by equipping (4.0.1) with kernels derived in Chapters 2 and 3.

The outline of this chapter is as follows. In Section 4.1, we consider the classical critical domain size problem. We study the minimal size for population persistence of a single good patch surrounded by bad habitat. We show that stability of the trivial solution may be studied as an eigenvalue problem and prove the existence of a dominant eigenpair by calling on and extending a number of previous results (Kot and Schaffer, 1986; VanKirk, 1995; Cantrell et al., 2012). In Section 4.1.1, we outline a procedure for obtaining the minimal patch size, and in Section 4.1.2, we study the dependence of this threshold on dispersal parameters in model (2.1.2). In Section 4.2, we analyze persistence conditions on an infinite periodic domain and generalize the results by Robbins (2004). The operator $Q$ is not necessarily compact in this case. We show that the persistence boundary is nonetheless given by an equivalent eigenvalue problem on a bounded set (Sections 4.2 and 4.3). Dependence of the persistence boundary on dispersal parameters in model (2.1.2) is illustrated in Section 4.4.1. We then illustrate how the interplay between dispersal ability and heterogeneity of the landscape affects persistence in Section 4.5.

\subsection{A Critical Domain Size Problem}

We consider a population residing in an infinite, one-dimensional landscape comprised

of a habitat patch, $\Omega=\left[-\frac{l_{1}}{2}, \frac{l_{1}}{2}\right]$, suitable for population growth that is adjacent to matrix habitat unsuitable for population growth. We determine the critical value, $l^{*}$, of $l_{1}$ such that the population persists precisely when $l_{1}>l^{*}$ (Skellam, 1951). Since we excluded an Allee effect, we study the linearized equation (1.3.2).

VanKirk and Lewis (1997) studied model (4.0.1) on a bounded domain and showed that the integral equation can be formulated as an equivalent boundary value 
problem. This technique also works for us by making use of the fact that $k(x, y)$ is the Green's function of the differential operator given by (2.2.6) and by applying the methods of Ludwig et al. (1979).

Applying (2.2.6) to (1.3.2) and following the same procedure as in (2.2.5), one obtains

$$
\begin{aligned}
\lambda \mathcal{L}[\phi(x)] & =\int_{-\infty}^{\infty} r_{0}(y) \phi(y) \mathcal{L}[k(x, y)] d y \\
& =-\int_{-\infty}^{\infty} r_{0}(y) \phi(y) \delta(x-y) d y=-r_{0}(x) \phi(x) .
\end{aligned}
$$

Expanding the left-hand side of (4.1.1) and simplifying, we obtain an ODE for the eigenvalue problem given by

$$
\frac{d^{2}}{d x^{2}}\left(\frac{\nu(x)}{\alpha(x)} \phi(x)\right)+\frac{\alpha(x)+\beta(x)}{\alpha(x)} \phi(x)(\omega \hat{r}(x)-1)=0
$$

where

$$
\omega=\frac{1}{\lambda}, \quad \hat{r}(x)=r_{0}(x) \frac{\alpha(x)}{\alpha(x)+\beta(x)},
$$

and $\lim _{x \rightarrow \pm \infty} \phi(x)=0$.

We assume that $\nu$ is a piecewise constant parameter function given by

$$
\nu(x)=\left\{\begin{array}{l}
\nu_{1},|x|<\frac{l_{1}}{2} \\
\nu_{2},|x|>\frac{l_{1}}{2},
\end{array}\right.
$$

and $\alpha$ and $\beta$ are analogously defined. Furthermore, we assume that the growth rate inside patch $\Omega$ is high so that $\hat{r}_{1}>1$, and outside $\Omega$ it is low, so that $0<\hat{r}_{2}<1$. Then equation (4.1.2) holds inside and outside $\Omega$; i.e.,

$$
\begin{aligned}
& \frac{d^{2}}{d x^{2}} \phi(x)+\mu_{1}^{2} \phi(x)\left[\omega \hat{r}_{1}-1\right]=0, \quad|x|<\frac{l_{1}}{2} \\
& \frac{d^{2}}{d x^{2}} \phi(x)-\mu_{2}^{2} \phi(x)\left[1-\omega \hat{r}_{2}\right]=0, \quad|x|>\frac{l_{1}}{2} \text {, }
\end{aligned}
$$


where $\mu_{i}=\sqrt{\frac{\alpha_{i}+\beta_{i}}{\nu_{i}}}, i=1,2$, while we have to impose appropriate interface conditions (see below) at $x= \pm \frac{l_{1}}{2}$.

The asymptotic conditions imposed on $\phi$ require that

$$
1-\frac{\hat{r}_{2}}{\lambda}>0 \Longrightarrow \hat{r}_{2}<\lambda
$$

and to satisfy the interface conditions, we require that

$$
\frac{\hat{r}_{1}}{\lambda}-1>0 \Longrightarrow \hat{r}_{1}>\lambda
$$

Therefore we see that (4.1.5) is solvable if and only if $\lambda \in\left(\hat{r}_{2}, \hat{r}_{1}\right)$.

The existence of a dominant eigenvalue and corresponding nonnegative eigenfunction for (4.1.5), and therefore (1.3.2), follows from the results of Brown et al. (1990) and the subsequent analysis of Cantrell et al. (2012). In the next section, we outline a procedure for obtaining a candidate for a positive solution $\phi$ of (4.1.5). Note that the solution to (1.3.1) with $N_{0}(x)=C \phi(x)$ for some $C>0$ is $N_{t}(x)=C \lambda^{t} \phi(x)$ so that (1.3.1) predicts growth if $\lambda>1$ and extinction if $\lambda<1$.

\subsubsection{Procedure for Obtaining the Dominant Eigenpair}

The system of ODEs given by (4.1.5) can be greatly simplified by making use of the spatial symmetry of the landscape about $x=0$. The equivalent system reads

$$
\begin{aligned}
& \frac{d^{2}}{d x^{2}} \phi(x)+\mu_{1}^{2} \phi(x)\left[\omega \hat{r}_{1}-1\right]=0, \quad 0<x<\frac{l_{1}}{2} \\
& \frac{d^{2}}{d x^{2}} \phi(x)-\mu_{2}^{2} \phi(x)\left[1-\omega \hat{r}_{2}\right]=0, \quad x>\frac{l_{1}}{2},
\end{aligned}
$$


subject to the following conditions:

$$
\begin{aligned}
& \phi^{\prime}(0)=0 \text {, by symmetry } \\
& \phi(x) \rightarrow 0 \text { as } x \rightarrow \infty \\
& \text { Interface conditions at } x=\frac{l_{1}}{2} .
\end{aligned}
$$

Case M: The interface conditions for $\phi$ are derived from (1.3.2), (2.4.8) and are given by

$$
\begin{aligned}
(1-z) \nu_{1} \alpha_{2} \phi\left(\frac{l_{1}^{-}}{2}\right) & =(1+z) \nu_{2} \alpha_{1} \phi\left(\frac{l_{1}^{+}}{2}\right) \\
\nu_{1} \alpha_{2} \phi^{\prime}\left(\frac{l_{1}^{-}}{2}\right) & =\nu_{2} \alpha_{1} \phi^{\prime}\left(\frac{l_{1}^{+}}{2}\right) .
\end{aligned}
$$

The only positive, bounded solutions of (4.1.8) for $x>\frac{l_{1}}{2}$ are of the form

$$
\phi(x)=A_{1} \exp \left(-\tau_{2} x\right)
$$

where $\tau_{2}=\sqrt{\mu_{2}^{2}\left(1-\omega \hat{r}_{2}\right)}$. Following the same steps as Ludwig et al. (1979), the set of interface conditions (4.1.10) can be recast as a boundary condition given by

$$
\begin{aligned}
\nu_{1} \alpha_{2} \phi^{\prime}\left(\frac{l_{1}^{-}}{2}\right)=\nu_{2} \alpha_{1} \phi^{\prime}\left(\frac{l_{1}^{+}}{2}\right) & =-\tau_{2} \nu_{2} \alpha_{1} A \exp \left(-\tau_{2} \frac{l_{1}^{+}}{2}\right) \\
& =-\tau_{2} \nu_{2} \alpha_{1} \phi\left(\frac{l_{1}^{+}}{2}\right)=-\tau_{2} \nu_{1} \alpha_{2} \frac{1-z}{1+z} \phi\left(\frac{l_{1}^{-}}{2}\right) .
\end{aligned}
$$

Therefore, the problem on an infinite domain has been recast as a boundary-value problem given by

$$
\begin{aligned}
& \frac{d^{2}}{d x^{2}} \phi(x)+\mu_{1}^{2} \phi(x)\left[\omega \hat{r}_{1}-1\right]=0, \quad 0 \leq x \leq \frac{l_{1}}{2} \\
& \phi^{\prime}\left(\frac{l_{1}}{2}\right)+\tau_{2} \frac{1+z}{1-z} \phi\left(\frac{l_{1}}{2}\right)=0, \quad \phi^{\prime}(0)=0 .
\end{aligned}
$$


The boundary condition at the origin requires

$$
\phi(x)=A_{2} \cos \left(\sqrt{\mu_{1}^{2}\left(\omega \hat{r}_{1}-1\right)} x\right) .
$$

Applying the boundary condition at $x=\frac{l_{1}}{2}$, the principal eigenvalue, $\omega=\frac{1}{\lambda}$, must satisfy the transcendental equation

$$
\tan \left(\frac{l_{1}}{2} \sqrt{\mu_{1}^{2}\left(\frac{\hat{r}_{1}}{\lambda}-1\right)}\right)=\bar{z} \sqrt{\frac{\mu_{2}^{2}\left(\lambda-\hat{r}_{2}\right)}{\mu_{1}^{2}\left(\hat{r}_{1}-\lambda\right)}} .
$$

Define the functions

$$
g(\lambda)=\tan \left(\frac{l_{1}}{2} \sqrt{\mu_{1}^{2}\left(\frac{\hat{r}_{1}}{\lambda}-1\right)}\right)
$$

and

$$
h(\lambda)=\bar{z} \sqrt{\frac{\mu_{2}^{2}\left(\lambda-\hat{r}_{2}\right)}{\mu_{1}^{2}\left(\hat{r}_{1}-\lambda\right)}} .
$$

The function $g$ has a vertical asymptote at $\lambda=\lambda^{*}$ where $\lambda^{*}$ is the solution of

$$
\frac{l_{1}}{2} \sqrt{\mu_{1}^{2}\left(\frac{\hat{r}_{1}}{\lambda}-1\right)}=\frac{\pi}{2}
$$

and the asymptotics of $g$ and $h$ are as follows

$$
\begin{aligned}
& \lim _{\lambda \rightarrow \hat{r}_{1}} g(\lambda)=0, \lim _{\lambda \rightarrow \hat{r}_{1}} h(\lambda)=\infty \\
& \lim _{\lambda \rightarrow \hat{r}_{2}} g(\lambda)=C_{g}, \lim _{\lambda \rightarrow \hat{r}_{2}} h(\lambda)=0 \\
& \lim _{\lambda \rightarrow \lambda^{*}} g(\lambda)=\infty, \quad \lim _{\lambda \rightarrow \lambda^{*}} h(\lambda)=C_{h},
\end{aligned}
$$

where $C_{g}$ and $C_{h}$ are positive constants. Thus, for $\lambda \in\left(\max \left\{\lambda^{*}, \hat{r}_{2}\right\}, \hat{r}_{1}\right), g$ is a monotone decreasing function and $h$ is a monotone increasing function. Therefore there is a unique $\lambda \in\left(\max \left\{\lambda^{*}, \hat{r}_{2}\right\}, \hat{r}_{1}\right)$ that solves (4.1.15). For this range of $\lambda$, the solution to (4.1.11) and (4.1.14) is positive and, hence, (4.1.8) admits a positive 
solution. Therefore, based on the analysis of Brown et al. (1990) and the subsequent analysis of Cantrell et al. (2012), $\phi$ is the unique positive eigenfunction corresponding to the dominant eigenvalue $\lambda$; see Appendix D.

Since stability of the trivial solution of (1.3.1) is lost as $\lambda$ passes through 1 , we set $\lambda=1$; solving equation (4.1.15) for $l_{1}$, we obtain the critical domain size

$$
l_{m}^{*}=\frac{2 \sqrt{\nu_{1}}}{\sqrt{\left(\alpha_{1}+\beta_{1}\right)\left(\hat{r}_{1}-1\right)}} \arctan \left(\bar{z} \sqrt{\frac{\nu_{1}\left(\alpha_{2}+\beta_{2}\right)\left(1-\hat{r}_{2}\right)}{\nu_{2}\left(\alpha_{1}+\beta_{1}\right)\left(\hat{r}_{1}-1\right)}}\right),
$$

where $\bar{z}=\frac{1-z}{1+z}$.

Following the same analysis with different interface conditions, the critical domain size for Case $\mathrm{S}$ is given by

$$
l_{s}^{*}=\frac{2 \sqrt{\nu_{1}}}{\sqrt{\left(\alpha_{1}+\beta_{1}\right)\left(\hat{r}_{1}-1\right)}} \arctan \left(\bar{z} \sqrt{\frac{\left(\alpha_{2}+\beta_{2}\right)\left(1-\hat{r}_{2}\right)}{\left(\alpha_{1}+\beta_{1}\right)\left(\hat{r}_{1}-1\right)}}\right),
$$

and for Case C by

$$
l_{c}^{*}=\frac{2 \sqrt{\nu_{1}}}{\sqrt{\left(\alpha_{1}+\beta_{1}\right)\left(\hat{r}_{1}-1\right)}} \arctan \left(\sqrt{\frac{\nu_{2}\left(\alpha_{2}+\beta_{2}\right)\left(1-\hat{r}_{2}\right)}{\nu_{1}\left(\alpha_{1}+\beta_{1}\right)\left(\hat{r}_{1}-1\right)}}\right) .
$$

\subsubsection{Qualitative Behavior of Critical Domain Size Formulae}

When motility inside and outside the habitat patch is the same $\left(\nu_{1}=\nu_{2}\right)$, the qualitative behavior of expressions (4.1.17)-(4.1.19) with respect to model parameters is identical. Figure 4.1 illustrates the dependence of the critical domain size on model parameters in the good patch and on the movement bias. The critical domain size is a convex, monotone-decreasing function of $\alpha_{1}$ and is a convex, monotone-increasing function of $\beta_{1}$; see Figures 4.1(a) and 4.1(b). As a function of the movement bias, the critical domain size is a concave, monotone-decreasing function, as can be observed in Figure 4.1(c). This agrees with the result that average dispersal success is a monotone 
increasing function of the bias; see Figure 3.9. As a function of parameters in bad patches, the critical domain size is a concave, monotone increasing function of $\beta_{2}$ and $\alpha_{2}$ (plot not shown).

The differences between equations (4.1.17)-(4.1.19) become apparent when the motility coefficients in the two patch types differ. The critical patch size is a monotonedecreasing function of $\nu_{2}$ for Case $\mathrm{M}$, monotone increasing for Case $\mathrm{C}$ and constant for Case S; see Figure 4.2(a). When $\nu_{2}$ is small, Case C corresponds to a significantly lower critical domain size as individuals have a low probability of emigrating to the matrix habitat. In Case M, low $\nu_{2}$ implies that individuals move slowly outside the good patch and therefore are more likely to settle there and not return to the good patch. As $\nu_{2}$ increases, individuals following Case $\mathrm{C}$ have an increased probability of leaving the good patch whereas individuals following Case $\mathrm{M}$ have a high movement rate outside the good patch and hence may return to the good patch before settling outside of it.

As a function of $\nu_{1}$, the critical patch size is a monotone increasing function for all cases. It is largest for Case $\mathrm{M}$ and smallest for Case $\mathrm{C}$ when $\nu_{1}>\nu_{2}$; the opposite is true when $\nu_{1}<\nu_{2}$ (Figure 4.2(b)). Note that the critical patch size is significantly smaller for Case $\mathrm{C}$ since individuals are less likely to leave the patch as $\nu_{1}$ increases. Note that the concavity/convexity properties of the critical domain size with respect to model parameters hold also when $\nu_{1}$ and $\nu_{2}$ are different.

Persistence conditions obtained here are closely related to those obtained by VanKirk and Lewis (1999). Those authors analyzed a critical domain size problem with a permeable boundary but did not consider mortality nor movement outside the patch. The permeability of the boundary was modeled by a parameter $\bar{P}$ that gives the rate per unit length at which an individual leaves the patch. Individuals tend to stay in the patch as $\bar{P} \rightarrow 0$, and they leave as $\bar{P} \rightarrow \infty$.

In Section 3.2, we showed that permeability can be related to movement bias and mean dispersal distance outside the patch in our model. In particular, we derived the 


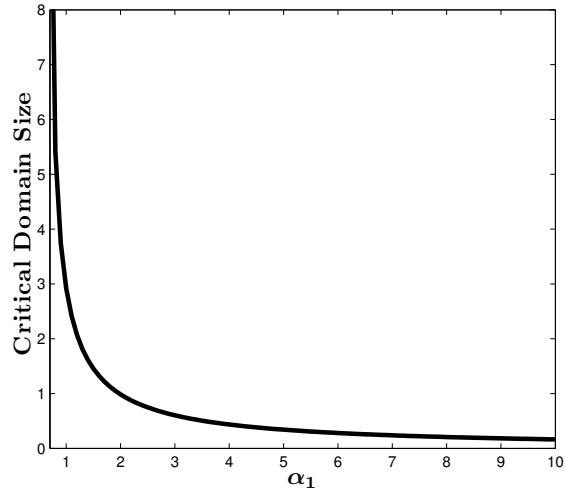

(a)

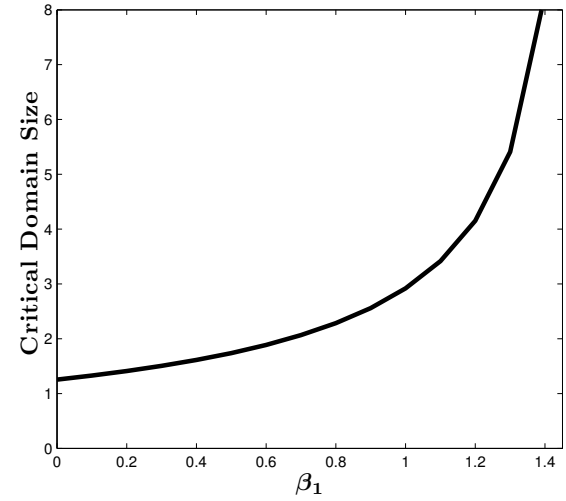

(b)

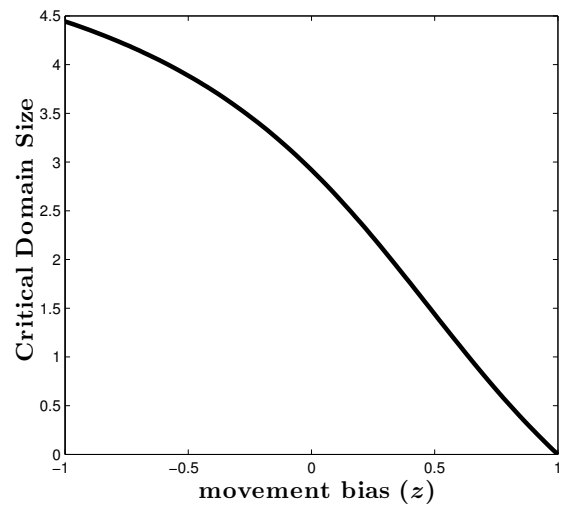

(c)

Figure 4.1: Qualitative behavior of equations (4.1.17)-(4.1.19) assuming $\nu_{1}=$ $\nu_{2}=1$, for (a) $\beta_{1}=1, z=0$, (b) $\alpha_{1}=1, z=0$, (c) $\alpha_{1}=1, \beta_{1}=1$. Fixed parameter values are: $r_{1}=2.5, r_{2}=0.6, \alpha_{2}=1$ and $\beta_{2}=1$. 


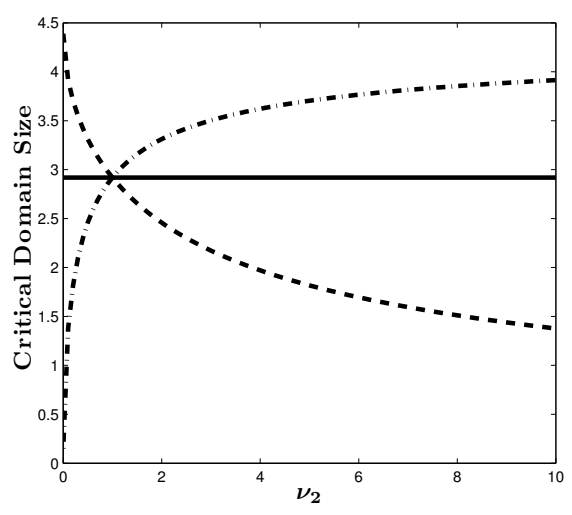

(a)

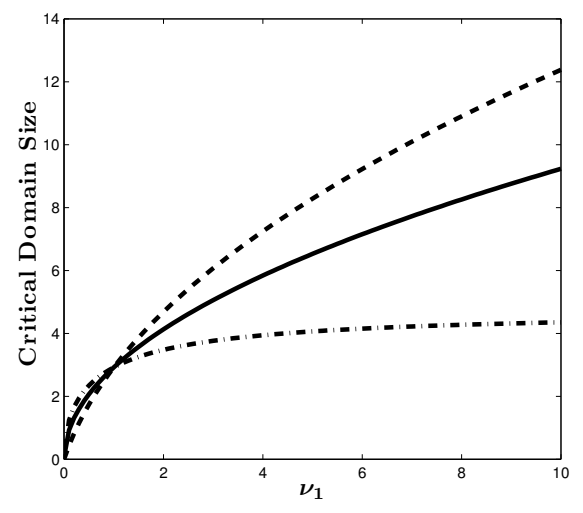

(b)

Figure 4.2: Qualitative behavior of the critical domain size assuming Case $\mathrm{S}$ (solid), M (dashed) and $\mathrm{C}$ (dashed-dot). For (a) $\nu_{1}=1$, (b) $\nu_{2}=1$. Fixed parameter values are: $\alpha_{1}=1, \alpha_{2}=1, \beta_{1}=1, \beta_{2}=1, r_{1}=2.5, r_{2}=0.6$, and $z=0$.

relation

$$
\bar{P}=\frac{1-z}{1+z} \sqrt{\frac{\alpha_{2}+\beta_{2}}{\nu_{2}}}
$$

From equation (4.1.20), we see that the effective rate of entering the patch increases as $\nu_{2}$ increases or $z \rightarrow 1$ and decreases as $\nu_{2}$ decreases or $z \rightarrow-1$. In Figure 4.3, we illustrate the effect of boundary permeability on the critical domain size for Case M. Observe that the permeability of the boundary is independent of the mean dispersal distance outside the patch if $z= \pm 1$. For intermediate values of the bias, we observe that the critical patch size decreases as permeability decreases.

\subsection{A Periodic Landscape}

In this section, we study the stability of the trivial solution of model (1.2.1) on an infinite, one-dimensional landscape that is periodically varying with good patches of length $l_{1}$ and bad patches of length $l_{2}$ so that the period of the landscape is $l=l_{1}+l_{2}$; 


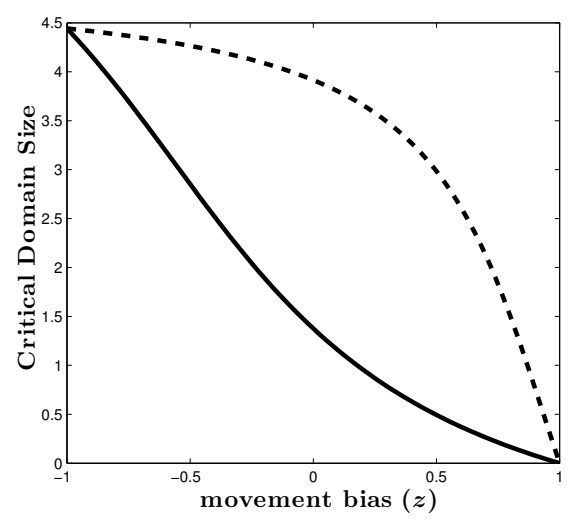

Figure 4.3: Qualitative behavior of the critical domain size with respect to patch preference for Case $\mathrm{M}$ assuming $\nu_{2}=0.1$ (dashed-dot) and $\nu_{2}=10$ (solid). Fixed parameter values are: $\alpha_{1}=1, \alpha_{2}=1, r_{2}=2.5, r_{2}=0.6$, $\nu_{1}=1, \beta_{1}=1, \beta_{2}=1$.

for example, see Figure 3.6. We show that the stability behavior may be studied as an eigenvalue problem, and we generalise the results of VanKirk (1995) and Robbins (2004) to prove the existence of a dominant eigenpair. We also give explicit formulae for the persistence boundary with respect to parameters.

Since the dispersal kernel is, in general, discontinuous, we choose the space of essentially bounded functions on $\mathbb{R}$. Since $k$ is nonnegative, the operator $Q$ given by (1.2.1) leaves the positive cone invariant. Thus we work on $\mathbb{L}_{+}^{\infty}(\mathbb{R})$, the set of nonnegative, bounded functions on $\mathbb{R}$. We assume that parameter functions are piecewise constant, where $\nu$ is given by

$$
\nu(x)=\left\{\begin{array}{l}
\nu_{1}, x \in\left(\frac{-l_{1}}{2}, \frac{l_{1}}{2}\right)+l \mathbb{Z} \\
\nu_{2}, x \in\left(\frac{l_{1}}{2}, l-\frac{l_{1}}{2}\right)+l \mathbb{Z}
\end{array}\right.
$$

and $\alpha, \beta$, and $\hat{r}$ are analogously defined. Furthermore, we assume that the growth rate inside good patches is high, so that $\hat{r}_{1}>1$, and in bad patches it is low, so that $0<\hat{r}_{2}<1$. 
To study the stability of the trivial solution of (1.2.1), we analyze the spectrum of its linearization. We use periodicity to recast $Q(1.2 .1)$ as an operator $\widehat{Q}$ on a compact set, so that its spectrum is well understood. We prove that the stability properties of the two operators are identical.

Lemma 4.2.1 (The kernel is shift invariant with period $l$ ) Assume that $\alpha, \beta$ and $\nu$ are l-periodic functions. Then the dispersal kernel given by (2.2.1) is shift invariant with period $l$; i.e., $k(x, y)=k(x+m l, y+m l)$ where $m \in \mathbb{Z}$.

\section{Proof:}

Recall, the general dispersal model (2.1.2) is given by

$$
\frac{\partial u}{\partial T}=\frac{\partial^{2}}{\partial x^{2}}(\nu(x) u)-(\alpha(x)+\beta(x)) u
$$

subject to periodic interface conditions and an initial condition. Let $u_{1}(x, T ; y)$ be the solution of (4.2.2) subject to the initial condition $u_{1}(x, 0 ; y)=\delta(x-y)$, and $u_{2}(x, T ; y)$ be the solution of (4.2.2) subject to the initial condition $u_{2}(x, 0 ; y)=\delta(x-(y+l))$. Let $v(x, T ; y)=u_{2}(x+l, T ; y)$ so that $v(x, 0 ; y)=\delta(x-y)=u_{1}(x, 0 ; y)$. Then $\frac{\partial}{\partial T}\left(u_{1}-v\right)=0$, which, by uniqueness of solutions, implies $u_{1}=v$ and, therefore, the solution to (4.2.2) is shift invariant with period $l$; i.e., $u(x, T ; y)=u(x+l, T ; y+l)$. Therefore, we have

$$
\begin{aligned}
k(x, y) & =\int_{0}^{\infty} \alpha(x) u(x, T ; y) d T \\
& =\int_{0}^{\infty} \alpha(x+l) u(x+l, T ; y+l) d T \\
& =k(x+l, y+l) ;
\end{aligned}
$$

i.e., the dispersal kernel is shift invariant with period $l$. 
Lemma 4.2.2 ( $Q$ is shift invariant with period $l$ ) Let the assumptions of Lemma 4.2.1 be satisfied and assume $f(N, x)$ is l-periodic in the second argument. Then $N(x+l)=N(x)$ implies $Q[N](x+l)=Q[N](x)$.

Proof: $\quad$ Consider the integral equation for $Q$ given by

$$
\begin{aligned}
Q[N](x) & =\int_{\mathbb{R}} f(N(y) ; y) k(x, y) d y, \\
& =\int_{\mathbb{R}} f(N(y+l) ; y+l) k(x+l, y+l) d y
\end{aligned}
$$

Let $\hat{y}=y+l$ to obtain

$$
\begin{aligned}
& =\int_{\mathbb{R}} f(N(\hat{y}) ; \hat{y}) k(x+l, \hat{y}) d \hat{y} \\
& =Q[N](x+l) .
\end{aligned}
$$

Note that the second equality in (4.2.4) is obtained using the periodicity of $N, f$, and $k$. In the following, we assume that the periodicity assumptions on parameter functions are satisfied.

As a consequence of Lemma 4.2.2, $Q$ maps the set of $l$-periodic, nonnegative, essentially bounded functions on $\mathbb{R}$ into itself. We denote this set as $\mathbb{L}_{l,+}^{\infty}$ and the restriction of $Q$ to this set as $Q_{l}$. By employing periodicity, the next lemma shows that fixed points of $Q_{l}$ are also fixed points of an operator on a bounded domain.

Lemma 4.2.3 (Restriction of $Q$ to a bounded domain) By periodically extending functions defined on $[0, l]$ or restricting l-periodic functions on $\mathbb{R}$ to $[0, l]$, fixed points of the integral operator $Q_{l}$ are precisely fixed points of the operator $\widehat{Q}_{l}$ defined on $\mathbb{L}_{+}^{\infty}([0, l])$ by

$$
\widehat{Q}_{l}[N(x)]:=\int_{0}^{l} f(N(y) ; y) \widehat{k}(x, y) d y,
$$


where

$$
\widehat{k}=\sum_{m=-\infty}^{\infty} k(x, y+m l), m \in \mathbb{Z} .
$$

Proof: Consider the equation for $Q_{l}$ given by

$$
\begin{aligned}
Q_{l}[N](x) & =\int_{-\infty}^{\infty} f(N(y) ; y) k(x, y) d y \\
& =\sum_{m=-\infty}^{\infty} \int_{m l}^{(m+1) l} f(N(y) ; y) k(x, y) d y
\end{aligned}
$$

Now, let $z=y-m l$ to obtain

$$
\begin{aligned}
& =\sum_{m=-\infty}^{\infty} \int_{0}^{l} f(N(z+m l) ; z+m l) k(x, z+m l) d z \\
& =\sum_{m=-\infty}^{\infty} \int_{0}^{l} f(N(z) ; z) k(x, z+m l) d z \\
& =\int_{0}^{l} f(N(z) ; z) \widehat{k}(x, z) d z,
\end{aligned}
$$

which is equation (4.2.5), as claimed. The interchange of the summation and integral operators above is justified by Tonelli's Theorem.

In order to make use of previous results by Krasnosel'skii (1964a) on nonlinear integral operators, we enlarge the set of functions to $\mathbb{L}_{+}^{2}([0, l])$, the set of nonnegative, square-integrable functions on $[0, l]$. By assumption, $f(N)$ is nonnegative and bounded, so that $f(N(x)) \in \mathbb{L}_{+}^{2}([0, l])$ if $N \in \mathbb{L}_{+}^{2}([0, l])$. The fact that the dispersal kernel is an $\mathbb{L}^{2}$ kernel is a result of the following lemma.

Lemma 4.2.4 (The dispersal kernel is essentially bounded) The dispersal kernel defined by (2.2.1) and obtained from (2.2.6) is essentially bounded; i.e., $\widehat{k}(x, y) \in$ $\mathbb{L}_{+}^{\infty}([0, l] \times[0, l])$. 
Proof: First we note that since $k$ is translation invariant with respect to multiples of $l$, we see that $k(x, y+l)=k(x-l, y)$. We may then rewrite the expression for $\widehat{k}$ in Lemma 4.2 .3 as

$$
\widehat{k}(x, y)=\sum_{m=-\infty}^{\infty} k(x, y+m l)=\sum_{m=-\infty}^{\infty} k(x-m l, y)
$$

It turns out that it is much simpler to work with a fixed release point versus a fixed arrival point.

For a fixed $y \in[0, l]$, we define

$$
\begin{aligned}
M_{m}^{g}=\max _{x \in\left[m l, m l+l_{1}\right]}\{k(x, y)\}, & A_{m}^{g}=\min _{x \in\left[m l, m l+l_{1}\right]}\{k(x, y)\} \\
M_{m}^{b}=\max _{x \in\left[m l+l_{1},(m+1) l\right]}\{k(x, y)\}, & A_{m}^{b}=\min _{x \in\left[m l+l_{1},(m+1) l\right]}\{k(x, y)\},
\end{aligned}
$$

where $M_{m}^{g, b}$ and $A_{m}^{g, b}$ denote the maximum and minimum value of the kernel obtained in the $m^{t h}$ good or bad patch, respectively. For $m \geq 1$, we claim

$$
M_{m}^{g} \leq A_{m-1}^{g}, \quad M_{m}^{b} \leq A_{m-1}^{b}
$$

and for $m \leq-1$, we claim

$$
M_{m}^{g} \leq A_{m+1}^{g}, \quad M_{m}^{b} \leq A_{m+1}^{b}
$$

At $x=y, k$ must be continuous with a positive slope as $x$ approaches $y$ from below and a negative slope from above (Keener, 2000). In order for (2.2.6) and the interface conditions to be satisfied, the function $x \mapsto k(x, y)$ is monotone-increasing within each patch for all $x<y$ and monotone-decreasing in each patch for all $x>y$. Therefore, the inequalities in (4.2.8) and (4.2.9) hold. We use $M_{m}^{g, b}$ and $A_{m}^{g, b}$ to show $\widehat{k}$ is essentially bounded. 
First, we show that the sum of minimum values in good and bad patches is bounded above by splitting the integral of $k$ over $\mathbb{R}$ into a sum of integrals over good and bad patches; i.e.,

$$
\begin{aligned}
1 \geq \int_{\mathbb{R}} k(x, y) d x & \geq \sum_{m=-\infty}^{\infty}\left(\int_{0}^{l_{1}} A_{m}^{g} d y+\int_{l_{1}}^{l} A_{m}^{b} d y\right) \\
& =\sum_{m=-\infty}^{\infty}\left(l_{1} A_{m}^{g}+l_{2} A_{m}^{b}\right) .
\end{aligned}
$$

Note that $\int_{\mathbb{R}} k(x, y) d x$ is the dispersal success function (see (3.4.1)) and is less than or equal to unity by definition.

Secondly, we make use of (4.2.6), (4.2.8), and (4.2.9) to obtain the following upper bound on $\widehat{k}$

$$
\begin{aligned}
\widehat{k}=\sum_{m=-\infty}^{\infty} k(x, y+m l) & =\sum_{m=-\infty}^{\infty} k(x-m l, y) \\
& \leq \sum_{m=-\infty}^{\infty}\left(\int_{0}^{l_{1}} M_{m}^{g} d y+\int_{l_{1}}^{l} M_{m}^{b}\right) \\
& \leq l_{1} M_{0}^{g}+l_{2} M_{0}^{b}+\sum_{m=-\infty}^{\infty}\left(l_{1} A_{m}^{g}+l_{2} A_{m}^{b}\right) .
\end{aligned}
$$

Now, we show that $M_{0}^{g}$ and $M_{0}^{b}$ are bounded. Let $u(x, T ; y)$ be the solution of (4.2.2). Let $\tilde{u}(x, T ; y)$ be the solution of $(4.2 .2)$ with $\beta_{1}=0$, and $z=1$ so that we obtain no-flux boundary conditions. Then, since there is no dispersal-induced mortality or boundary-loss, $u(x, T ; y) \leq \tilde{u}(x, T ; y)$ for all $(x, y) \in\left[0, l_{1}\right] \times\left[0, l_{1}\right]$ and $T \geq 0$. VanKirk (1995) showed that the dispersal kernel obtained from $\tilde{u}$ is given by

$$
\begin{aligned}
\tilde{k}(x, y)=1 & +\sum_{n=1}^{\infty} \frac{2 \frac{\nu_{1}}{\alpha_{1}} l_{1}^{2}}{4 n^{2} \pi^{2} \frac{\nu_{1}}{\alpha_{1}} l_{1}^{2}} \cos (2 n \pi x) \cos (2 n \pi y) \\
& +\sum_{n=1}^{\infty} \frac{2 \frac{\nu_{1}}{\alpha_{1}} l_{1}^{2}}{(2 n-1)^{2} \pi^{2} \frac{\nu_{1}}{\alpha_{1}} l_{1}^{2}} \sin ((2 n-1) \pi x) \sin ((2 n-1) \pi y) .
\end{aligned}
$$


Therefore we see that the kernel is bounded above independent of $x$ and $y$; i.e..

$$
\tilde{k}(x, y) \leq 1+\sum_{n=1}^{\infty}\left(\frac{2 \frac{\nu_{1}}{\alpha_{1}} l_{1}^{2}}{(2 n-1)^{2} \pi^{2} \frac{\nu_{1}}{\alpha_{1}} l_{1}^{2}}+\frac{2 \frac{\nu_{1}}{\alpha_{1}} l_{1}^{2}}{4 n^{2} \pi^{2} \frac{\nu_{1}}{\alpha_{1}} l_{1}^{2}}\right)
$$

Since the above sum is convergent, $M_{0}^{g}$ in $(4.2 .11)$ is bounded by a finite value that does not depend on $x$ or $y$. Using an analogous argument, one may show that $M_{0}^{b}$ in (4.2.11) is also bounded by a finite value that does not depend on $x$ or $y$.

Since $\sum_{m=-\infty}^{\infty}\left(l_{1} A_{m}^{g}+l_{2} A_{m}^{b}\right) \leq 1$ by $(4.2 .10), \widehat{k}$ is bounded for all $(x, y) \in$ $[0, l] \times[0, l]$; i.e., $\widehat{k}$ is an essentially bounded kernel on $[0, l] \times[0, l]$.

We now employ the results of VanKirk (1995) to prove the existence of a dominant eigenpair for (4.2.5). Briefly, VanKirk (1995) applied several results of Krasnosel'skii (1964a) to show that $\widehat{Q}_{l}$ and its linearization are both completely continuous operators. The existence of a positive eigenvalue and nonnegative eigenfunction then follow from the Krein-Rutman Theorem (Krein and Rutman, 1950). The simplicity and dominance of the eigenvalue, as well as, the uniqueness (up to constant multiple) of the non-negative eigenfunction, follow from Krasnosel'skii (1964a). The details of this analysis is presented in Appendix E and largely follow the analysis in Appendices B.6.1-B.6.4 of VanKirk (1995). For ease of referral, we summarize the main results required for our analysis.

Property 4.2.5 The nonlinear operator $\widehat{Q}_{l}: \mathbb{L}_{+}^{2}(\Omega) \rightarrow \mathbb{L}_{+}^{2}(\Omega)$ as defined by (4.2.5) is completely continuous, Fréchet differentiable with respect to $\mathbb{L}_{+}^{2}(\Omega)$ at $N^{*}(x)=0$, and its Fréchet derivative is the completely continuous operator given by

$$
B[N](x):=\int_{0}^{l} r_{0}(y) N(y) \widehat{k}(x, y) d y
$$


where

$$
r_{0}(y)=\frac{d f}{d N}(0 ; y)
$$

Property 4.2.6 Under the assumptions of this section, the linear operator $B$ has a positive, simple eigenvalue $\lambda$ corresponding to a nonnegative eigenfunction $\phi(x)$. This eigenvalue is strictly larger in modulus than any other eigenvalue of $B$, and its eigenfunction is the only (up to a constant multiple) nonnegative eigenfunction of $B$.

\subsection{Stability Equivalence of Operators}

In this section, we show that stability properties of the trivial solution for $Q$ on $\mathbb{L}_{+}^{\infty}(\mathbb{R})$ in (1.2.1) and $\widehat{Q}_{l}$ on $\mathbb{L}_{+}^{2}([0, l])$ in (4.2.5) are equivalent. We formulate the statements for local asymptotic stability, but it is clear from the proofs that the same statements are true for stability. The proof is broken down in a series of lemmas.

Theorem 4.3.1 The trivial solution is locally asymptotically stable (l.a.s.) for $Q$ in $\mathbb{L}_{+}^{\infty}(\mathbb{R})$ if and only if the trivial solution is l.a.s. for $\widehat{Q}_{l}$ in $\mathbb{L}_{+}^{2}([0, l])$.

Lemma 4.3.2 The trivial solution is l.a.s. in $\mathbb{L}_{+}^{\infty}(\mathbb{R})$ if and only if the trivial solution is l.a.s. in $\mathbb{L}_{l,+}^{\infty}(\mathbb{R})$.

Proof: Assume that zero is l.a.s. in $\mathbb{L}_{+}^{\infty}(\mathbb{R})$. Then, for all $\epsilon>0$, there exists some $\varrho>0$ such that, for every bounded function $N$ with $\|N\|_{\infty}<\varrho$, we have $\left\|Q^{t}[N]\right\|_{\infty}<\epsilon$ for all $t>0$ and $\left\|Q^{t}[N]\right\|_{\infty} \rightarrow 0$ as $t \rightarrow \infty$. Since $\mathbb{L}_{l,+}^{\infty}(\mathbb{R}) \subset \mathbb{L}_{+}^{\infty}(\mathbb{R})$, the same is true in $\mathbb{L}_{l,+}^{\infty}(\mathbb{R})$.

Assume that zero is l.a.s. in $\mathbb{L}_{l,+}^{\infty}(\mathbb{R})$. Then, for all $\epsilon>0$, there exists some $\hat{\varrho}>0$ such that, for every $l$-periodic function $\widehat{N}$ with $\|\widehat{N}\|_{\infty}<\hat{\varrho}$, we have $\left\|Q_{l}^{t}[\widehat{N}]\right\|_{\infty}<\epsilon$ 
for all $t>0$ and $\left\|Q_{l}^{t}[\widehat{N}]\right\|_{\infty} \rightarrow 0$ as $t \rightarrow \infty$. Since $f$ is monotone in $N$, the operator $Q_{l}$ is monotone. Hence, for any bounded function $N$ with $\|N\|_{\infty}<\hat{\varrho} / 2$, we have $\left\|Q^{t}[N]\right\|_{\infty} \leq\left\|Q_{l}^{t}[\hat{\varrho}]\right\|_{\infty}<\epsilon$ for all $t>0$ and $\left\|Q^{t}[N]\right\|_{\infty} \leq\left\|Q_{l}^{t}[\hat{\varrho}]\right\|_{\infty} \rightarrow 0$ as $t \rightarrow \infty$. Therefore the reverse direction holds.

Lemma 4.3.3 The trivial solution is l.a.s. in $\mathbb{L}_{l,+}^{\infty}(\mathbb{R})$ if and only if the trivial solution is l.a.s. in $\mathbb{L}_{+}^{\infty}([0, l])$.

Proof: $\quad$ From the definition of $\widehat{Q}_{l}(4.2 .5)$ and the proof of Lemma 4.2.3, it is clear that the operators $Q_{l}$ and $\widehat{Q}_{l}$ are equivalent provided $\widehat{Q}_{l}$ is periodically extended to $\mathbb{R}$ or $Q_{l}$ is restricted to $[0, l]$.

The next step uses the assumption that the growth function is bounded by its linearization at zero; i.e., $f(N, x) \leq f^{\prime}(0 ; x) N=r_{0}(x) N$. In fact, it is sufficient that this bound holds for $N$ near zero. In particular, the following lemma also holds if we replace the Beverton-Holt-type function $f$ with the Ricker function for $f$. We denote the maximum of the linearization by $R_{0}=\max _{0 \leq x \leq l} r_{0}(x)$.

Lemma 4.3.4 The trivial solution is l.a.s in $\mathbb{L}_{+}^{\infty}([0, l])$ if and only if the trivial solution is l.a.s. in $\mathbb{L}_{+}^{2}([0, l])$.

Proof: To prove this lemma, we must show that:

(1) For any $N \in \mathbb{L}_{+}^{2}([0, l]), \widehat{Q}_{l}[N](x)<R_{0} \widehat{K} \sqrt{l}\|N\|_{2}$.

(2) For any $N \in \mathbb{L}_{+}^{\infty}([0, l]),\|N\|_{2} \leq \sqrt{l}\|N\|_{\infty}$. 
The claim in (1) can be seen as follows.

$$
\begin{aligned}
\widehat{Q}_{l}[N](x) & =\int_{0}^{l} f(N(y) ; y) \widehat{k}(x, y) d y \leq R_{0} \int_{0}^{l} N(y) \widehat{k}(x, y) d y \\
& \leq R_{0}\|\widehat{k}(x, \cdot)\|_{\infty} \int_{0}^{l} N(y) d y \leq R_{0} \widehat{K} \sqrt{l}\|N\|_{2}
\end{aligned}
$$

where $\widehat{K}=\|\widehat{k}\|_{\infty}$.

Now, since $\mathbb{L}_{+}^{\infty}([0, l]) \subset \mathbb{L}_{+}^{2}([0, l])$, for any $N \in \mathbb{L}_{+}^{\infty}([0, l])$ we obtain

$$
\begin{aligned}
\|N\|_{2}=\left(\int_{0}^{l}|N|^{2} d x\right)^{1 / 2} & \leq\left(\int_{0}^{l}\|N\|_{\infty}^{2} d x\right)^{1 / 2} \\
& =\|N\|_{\infty}\left(\int_{0}^{l} d x\right)^{1 / 2}=\sqrt{l}\|N\|_{\infty}
\end{aligned}
$$

which proves $(2)$.

We can now proceed to prove the lemma. Assume that zero is stable in $\mathbb{L}_{+}^{\infty}([0, l])$. By (4.3.1), for any $\hat{N} \in \mathbb{L}_{+}^{2}([0, l])$, we have $\left\|\widehat{Q}_{l}[\hat{N}]\right\|_{\infty}<R_{0} \widehat{K} \sqrt{l}\|\hat{N}\|_{2}$. Thus $\widehat{Q}_{l}^{t}[\hat{N}] \in$ $\mathbb{L}_{+}^{\infty}([0, l])$ and, by assumption, for all $\epsilon>0$ there exists a $\hat{\varrho}>0$ such that $\|\hat{N}\|_{\infty}<\hat{\varrho}$ implies $\left\|\widehat{Q}_{l}^{t}[\hat{N}]\right\|_{\infty}<\epsilon$ and $\left\|\widehat{Q}_{l}^{t}[\hat{N}]\right\|_{\infty} \rightarrow 0$ as $t \rightarrow \infty$. Furthermore by (4.3.2) we have $\left\|\widehat{Q}_{l}^{t}[\hat{N}]\right\|_{2} \leq \sqrt{l}\left\|\widehat{Q}_{l}^{t}[\hat{N}]\right\|_{\infty}$ and the forward direction holds.

To prove the reverse direction, assume zero is stable in $\mathbb{L}_{+}^{2}([0, L])$. By (4.3.1), for any $N \in \mathbb{L}_{+}^{\infty}([0, l])$ we have that $\widehat{Q}[N] \in \mathbb{L}_{+}^{\infty}([0, l])$ and $\left\|\widehat{Q}_{l}^{t}[N]\right\|_{\infty} \leq R_{0} \widehat{K} \sqrt{l}\left\|\widehat{Q}_{l}^{t}[N]\right\|_{2}$. By assumption, for all $\epsilon>0$ there exists a $\varrho>0$ such that $\|N\|_{2}<\varrho$ implies $\left\|\widehat{Q}_{l}^{t}[N]\right\|_{2}<\epsilon$ and $\left\|\widehat{Q}_{l}^{t}[N]\right\|_{2} \rightarrow 0$ as $t \rightarrow \infty$. Therefore the reverse direction holds.

Therefore, by applying Lemmas 4.3.2, 4.3.3, and 4.3.4 the proof of Theorem 4.3.1 is complete. 


\subsection{Application: Critical Size of Good Patches}

Recall that the eigenvalue problem for (4.2.13) is given by

$$
\lambda \phi(x)=\int_{0}^{l} r_{0}(y) \phi(y) \widehat{k}(x, y) d y
$$

Using the definition of $\widehat{k}(x, y)$, we extend $\phi$ periodically to $\mathbb{R}$; i.e., $\phi(x)=\phi(x+l)$, and obtain

$$
\lambda \phi(x)=\int_{-\infty}^{\infty} r_{0}(y) \phi(y) k(x, y) d y .
$$

The integral equation for $\phi$ can be recast as an equivalent ODE by using the fact that $k$ is the Green's function of the differential operator (2.2.6). Applying (2.2.6) to (4.4.2) and following the same procedure as in (4.1.1), we obtain

$$
\frac{\partial^{2}}{\partial x^{2}}\left(\frac{\nu(x)}{\alpha(x)} \phi(x)\right)+\frac{\alpha(x)+\beta(x)}{\alpha(x)}(\omega \hat{r}(x)-1) \phi(x)=0
$$

where $\omega=\frac{1}{\lambda}$, and $\hat{r}(x)=r_{0}(x) \frac{\alpha(x)}{\alpha(x)+\beta(x)}$. We also require appropriate interface conditions for (4.4.3), and these may be derived from equation (4.4.2) in an analogous manner to (4.1.10).

The class of homogeneous, linear, second-order differential equations with real, periodic coefficients is commonly referred to as Hill's equation (Magnus and Winkler, 1966), and thus, (4.4.3) is a form of Hill's equation. Theorem 2.1 and Lemma 2.6 from Magnus and Winkler (1966) show that Hill's equation possesses a simple, minimum, finite eigenvalue, $\omega$. Furthermore, $\omega$ corresponds to the simple, positive, maximum eigenvalue for the IDE given by $\lambda$. Since stability of the trivial solution of (4.2.13) is determined by the dominant eigenvalue and lost as $\lambda$ passes through 1 , we find the stability boundary by determining the nontrivial solution to (4.4.3) when $\omega=1$.

Applying the definitions of $\alpha, \beta, \nu$ and $\hat{r}$ in (4.2.1) to the eigenvalue equation 
(4.4.3), we obtain the following periodic system of ODEs for $\phi$

$$
\begin{aligned}
\frac{d^{2}}{d x^{2}} \phi(x)+\mu_{1}^{2} \phi(x)\left[\omega \hat{r}_{1}-1\right]=0, & x \in\left(\frac{-l_{1}}{2}, \frac{l_{1}}{2}\right)+l \mathbb{Z} \\
\frac{d^{2}}{d x^{2}} \phi(x)-\mu_{2}^{2} \phi(x)\left[1-\omega \hat{r}_{2}\right] & =0, \quad x \in\left(\frac{l_{1}}{2}, l-\frac{l_{1}}{2}\right)+l \mathbb{Z},
\end{aligned}
$$

with appropriate interface conditions (see below).

Since (4.4.4) is periodic, we may restrict the analysis to one period. Furthermore, since (4.4.4) is symmetric with respect to $x \rightarrow-x$, we only need to consider $\left[0, \frac{l}{2}\right]$. We study the resulting system

$$
\begin{aligned}
& \frac{d^{2}}{d x^{2}} \phi(x)+\mu_{1}^{2} \phi(x)\left[\omega \hat{r}_{1}-1\right]=0, \quad x \in\left(0, \frac{l_{1}}{2}\right) \\
& \frac{d^{2}}{d x^{2}} \phi(x)-\mu_{2}^{2} \phi(x)\left[1-\omega \hat{r}_{2}\right]=0, \quad x \in\left(\frac{l_{1}}{2}, \frac{l}{2}\right) \text {, }
\end{aligned}
$$

with boundary conditions (from symmetry)

$$
\phi^{\prime}(0)=0, \quad \phi^{\prime}\left(\frac{l_{1}}{2}\right)=0
$$

and interface conditions given by Case M

$$
\begin{aligned}
(1-z) \nu_{1} \alpha_{2} \phi\left(\frac{l_{1}^{-}}{2}\right) & =(1+z) \nu_{2} \alpha_{1} \phi\left(\frac{l_{2}^{+}}{2}\right) \\
\nu_{1} \alpha_{2} \phi^{\prime}\left(\frac{l_{1}^{-}}{2}\right) & =\nu_{2} \alpha_{1} \phi^{\prime}\left(\frac{l_{2}^{+}}{2}\right) .
\end{aligned}
$$

The general solution of (4.4.5) is given by

$$
\begin{aligned}
& \phi(x)=A \cos \left(\tau_{1} x\right)+B \sin \left(\tau_{1} x\right), x \in\left(0, \frac{l_{1}}{2}\right) \\
& \phi(x)=C \cosh \left(\tau_{2}\left(\frac{l}{2}-x\right)\right)+D \sinh \left(\tau_{2}\left(\frac{l}{2}-x\right)\right), x \in\left(\frac{l_{1}}{2}, \frac{l}{2}\right)
\end{aligned}
$$


where $\tau_{1}=\sqrt{\mu_{1}^{2}\left(\omega \hat{r}_{1}-1\right)}$ and $\tau_{2}=\sqrt{\mu_{2}^{2}\left(1-\omega \hat{r}_{2}\right)}$. Applying the boundary conditions (4.4.6), we require that $B=D=0$. Interface conditions (4.4.7) translate into a system of linear equations for $A$ and $C$, given by

$$
\begin{aligned}
A \nu_{1} \alpha_{2}(1-z) \cos \left(\frac{\tau_{1} l_{1}}{2}\right)-C \nu_{2} \alpha_{1}(1+z) \cosh \left(\frac{\tau_{2} l_{2}}{2}\right) & =0 \\
-A \nu_{1} \alpha_{2} \tau_{1} \sin \left(\frac{\tau_{1} l_{1}}{2}\right)+C \nu_{2} \alpha_{1} \tau_{2} \sinh \left(\frac{\tau_{2} l_{2}}{2}\right) & =0
\end{aligned}
$$

A nontrivial solution for $A, C$ requires the minimal eigenvalue, $\omega$, to satisfy

$$
\tan \left(\frac{\tau_{1} l_{1}}{2}\right)=\frac{\bar{z} \tau_{2}}{\tau_{1}} \tanh \left(\frac{\tau_{2} l_{2}}{2}\right)
$$

where $\bar{z}=\frac{1-z}{1+z}$. Upon setting $\omega=1$ and re-arranging equation (4.4.10) for $l_{1}$, we obtain the critical size of good patches as

$$
l_{1, M}^{*}=\frac{2}{\tau_{1}} \arctan \left(\frac{\bar{z} \tau_{2}}{\tau_{1}} \tanh \left(\frac{\tau_{2} l_{2}}{2}\right)\right)
$$

The same steps applied to the interface conditions for Cases $\mathrm{S}$ and $\mathrm{C}$ lead to the critical sizes

$$
\begin{aligned}
& l_{1, S}^{*}=\frac{2}{\tau_{1}} \arctan \left(\frac{\bar{z} \tau_{2}}{\tau_{1}} \sqrt{\frac{\nu_{2}}{\nu_{1}}} \tanh \left(\frac{\tau_{2} l_{2}}{2}\right)\right) \\
& l_{1, C}^{*}=\frac{2}{\tau_{1}} \arctan \left(\frac{\nu_{2}}{\nu_{1}} \frac{\tau_{2}}{\tau_{1}} \tanh \left(\frac{\tau_{2} l_{2}}{2}\right)\right) .
\end{aligned}
$$

Before proceeding, we briefly discuss the relation between the dominant eigenvalue, $\lambda$, and the spatial average of the reproductive rate of settled individuals (4.1.3), extending the considerations by VanKirk and Lewis (1997).

The equation for $\lambda$ follows from equation (4.4.3) and is given by

$$
\lambda\left(1-\frac{s(x)}{\phi(x)}\left(\frac{\nu(x) \phi(x)}{\alpha(x)}\right)^{\prime \prime}\right)=\hat{r}(x)
$$

where $s(x)=\frac{\alpha(x)}{\alpha(x)+\beta(x)}$. The substitution $\psi(x):=\frac{\nu(x) \phi(x)}{\alpha(x)}$ simplifies equation (4.4.13) 
to

$$
\lambda\left(1-g(x) \frac{\psi^{\prime \prime}(x)}{\psi(x)}\right)=\hat{r}(x)
$$

where $g(x)=\frac{\nu(x)}{\alpha(x)+\beta(x)}=\frac{1}{\mu^{2}(x)}$.

The integration of (4.4.14) is greatly simplified by making the substitution

$$
h(x):=\frac{\psi^{\prime}(x)}{\psi(x)}=\frac{d}{d x} \ln (\psi(x))
$$

so that $\frac{\psi^{\prime \prime}(x)}{\psi(x)}=h^{\prime}(x)+h^{2}(x)$. Applying (4.4.15) to the left-hand side of (4.4.14), we obtain

$$
\lambda \int_{0}^{l}\left(1-g(x)\left(h^{\prime}(x)+h^{2}(x)\right)\right) d x=\int_{0}^{l} \hat{r}(x) d x .
$$

Functions $g$ and $h$ are continuously differentiable everywhere in $[0, l]$ except at the two interfaces $x=l_{1} / 2$ and $x=l_{1} / 2+l_{2}$. Thus, to evaluate the left-hand side of (4.4.16), we break up the integral into three pieces such that $g$ and $h^{\prime}$ are continuous on each. Note that function $g$ is of the form

$$
g(x)=\left\{\begin{array}{l}
\frac{1}{\mu_{1}^{2}}, \quad 0 \leq x<\frac{l_{1}}{2} \\
\frac{1}{\mu_{2}^{2}}, \frac{l_{1}}{2}<x<\frac{l_{1}}{2}+l_{2} \\
\frac{1}{\mu_{1}^{2}}, \frac{l_{1}}{2}+l_{2}<x \leq l .
\end{array}\right.
$$

Then we obtain

$$
\begin{aligned}
\int_{0}^{l} g(x) h^{\prime}(x) d x & =\frac{h\left(\frac{l_{1}^{-}}{2}\right)}{\mu_{1}^{2}}-\frac{h(0)}{\mu_{1}^{2}}+\frac{h\left(\frac{l_{1}}{2}+l_{2}^{-}\right)}{\mu_{2}^{2}}-\frac{h\left(\frac{l_{1}^{+}}{2}\right)}{\mu_{2}^{2}}+\frac{h(l)}{\mu_{1}^{2}}-\frac{h\left(\frac{l_{1}}{2}+l_{2}^{+}\right)}{\mu_{1}^{2}} \\
& =\left[h\left(\frac{l_{1}^{-}}{2}\right) / \mu_{1}^{2}-h\left(\frac{l_{1}^{+}}{2}\right) / \mu_{2}^{2}\right]-\left[h\left(\frac{l_{1}}{2}+l_{2}^{+}\right) / \mu_{1}^{2}-h\left(\frac{l_{1}}{2}+l_{2}^{-}\right) / \mu_{2}^{2}\right]
\end{aligned}
$$

where $\frac{h(l)}{\mu_{1}^{2}}-\frac{h(0)}{\mu_{1}^{2}}=0$ by periodicity of $h$. The two quantities in brackets, which represent the jumps at the discontinuities, are equal by symmetry, so the integral is 
zero. Thus the left-hand side of (4.4.16) simplifies to

$$
\lambda \int_{0}^{l}\left(1-g(x)\left(h^{\prime}(x)+h^{2}(x)\right)\right) d x=\lambda\left(l-\int_{0}^{l} g(x) h^{2}(x) d x\right) \leq \lambda l .
$$

Therefore, since $\lambda>0$, we obtain

$$
0<\int_{0}^{l}\left(1-g(x) \frac{\psi^{\prime \prime}(x)}{\psi(x)}\right) d x \leq l
$$

Therefore, the dominant eigenvalue (4.4.16) satisfies the inequality

$$
\lambda l \geq \int_{0}^{l} \hat{r}(x) d x
$$

Equation (4.4.20) thus implies that if

$$
\frac{1}{l} \int_{0}^{l} \hat{r}(x) d x>1
$$

then $\lambda>1$ so that the population always persists. Therefore, sufficient conditions for persistence depend on the spatial average of the reproductive rate $\hat{r}$ of settled individuals and not just $r$ itself.

\subsubsection{Qualitative Behavior of Critical Size of Good Patches}

The qualitative behavior of the critical sizes $l_{1, M}^{*}$ and $l_{1, S}^{*}$ in (4.4.11) and (4.4.12) with respect to changes in parameters is the same assuming $\nu_{1}$ equals $\nu_{2}$. In general, the monotonicity properties of the critical domain size with respect to model parameters discussed in Section 4.1 hold true for (4.4.11)-(4.4.12). However, the size of the persistence/extinction region observed in Figures 4.1 and 4.2 depends on the size of bad patches, as well as, dispersal behavior in either patch type.

Expressions (4.4.11)-(4.4.12) are quantitatively different when the motility coefficients in the two patch types are different. The plots in Figure 4.4(a) show that $l_{1, M}^{*}$ 


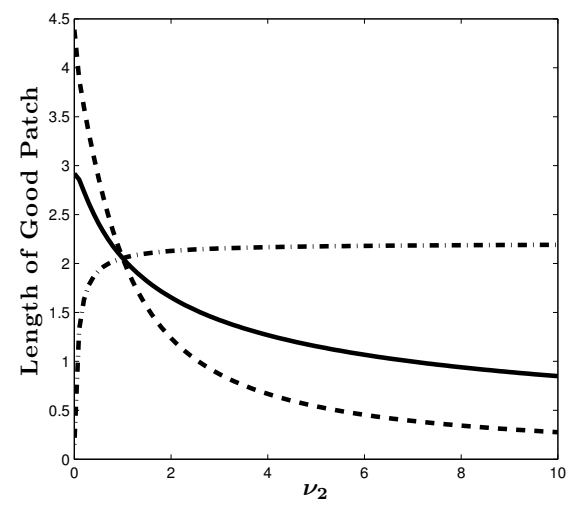

(a)

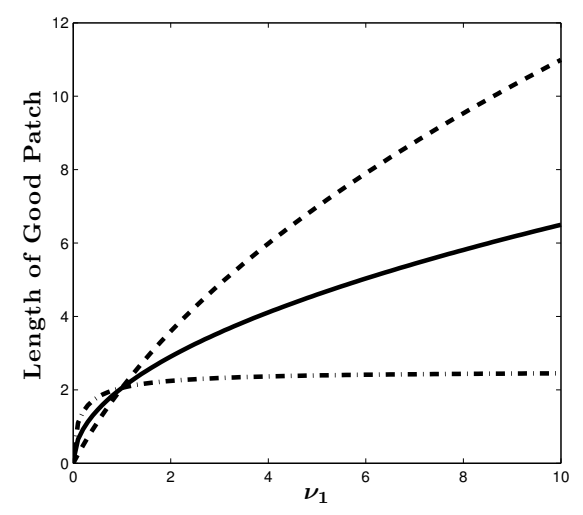

(b)

Figure 4.4: Critical size of good patches ((4.4.11)-(4.4.12)) assuming the interface conditions are given by Case M (dashed), S (solid), and C (dasheddot) for (a) increasing values of $\nu_{2}, \nu_{1}=1$ and (b) increasing values of $\nu_{1}$, $\nu_{2}=1$. Fixed parameter values are: $\alpha_{1}=1, \alpha_{2}=1, \beta_{1}=1, \beta_{2}=1, l_{2}=2$, $r_{1}=2.5, r_{2}=0.6$, and $z=0$.

and $l_{1, S}^{*}$ are monotone decreasing functions of $\nu_{2}$. This behavior agrees with our previous result that average dispersal success is an increasing function of $\nu_{2}$ (see Figure 3.9). However, $l_{1, C}^{*}$ is a monotone increasing function. This discrepancy occurs since individuals become more likely to enter a bad patch as $\nu_{2}$ increases. The value of $l_{1}^{*}$ is largest for Case $\mathrm{M}$ when $\nu_{1}>\nu_{2}$ and is largest for Case $\mathrm{C}$ when $\nu_{1}<\nu_{2}$. Figure 4.4(b) shows that $l_{1}^{*}$ is a monotone increasing function of $\nu_{1}$, regardless of movement behavior at an interface, agreeing with our previous result that the average dispersal success is a decreasing function of $\nu_{1}$ (see Figure 3.9).

The critical sizes of good patches for all three cases are increasing functions of $l_{2}$ and their values at $l_{2}=\infty$ correspond to the critical domain sizes for a single patch landscape as given in expressions (4.1.17)-(4.1.19), respectively. Thus, it follows that the values of (4.4.11)-(4.4.12) should be smaller than their corresponding values for a single patch landscape given by (4.1.17)-(4.1.19), and this can be observed from Figures 4.2 and 4.4 . 


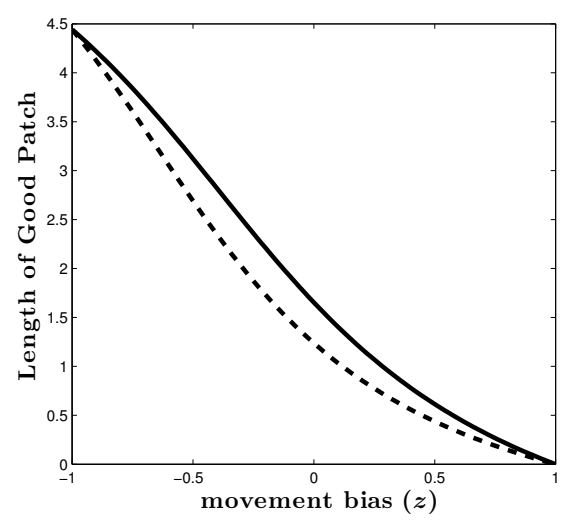

Figure 4.5: Critical size of good patches as a function of movement bias at an interface, assuming the interface conditions are given by Case $\mathrm{M}$ (dashed) and Case $\mathrm{S}$ (solid). Fixed parameter values are: $\nu_{1}=1, \nu_{2}=2, \alpha_{1}=1$, $\alpha_{2}=1, \beta_{1}=1, \beta_{2}=1, l_{2}=1, r_{1}=2.5$, and $r_{2}=0.6$.

The amount of favorable habitat required for persistence is a decreasing function of the movement bias parameter, $z$, regardless of behavior at an interface as shown in Figure 4.5. For a strong bias towards good patches, $z \approx 1$, the population persists regardless of the size of bad patches since individuals are highly unlikely to leave any good patch. If the movement bias is toward bad patches, the size of good patches must be large enough to ensure that individuals do not reach any interface, and settle in any of the bad patches.

As a last example, we examine the relationship between ((4.4.11)-(4.4.12)) and mortality during dispersal. Since foraging theory suggests individuals move slower in good than bad habitat, we plot ((4.4.11)-(4.4.12)) assuming $\nu_{2}>\nu_{1}$, and increasing values of $\beta_{2}$ in Figure 4.6. Observe that the persistence boundary is a concave, increasing function of $\beta_{2}$, regardless of the interface conditions. The value is largest for Case $\mathrm{C}$ since individuals are more likely to move into a bad patch as $\nu_{2}$ increases. However, if we assume individuals move faster in good habitat, then the value of $l_{1}^{*}$ significantly increases as the hostility of bad patches increases (plot not shown). In Figure 4.6(b), we observe that if the quality of good patches significantly decreases, 


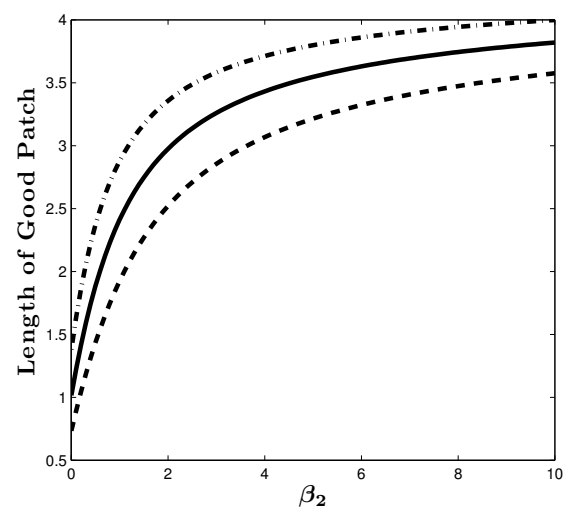

(a)

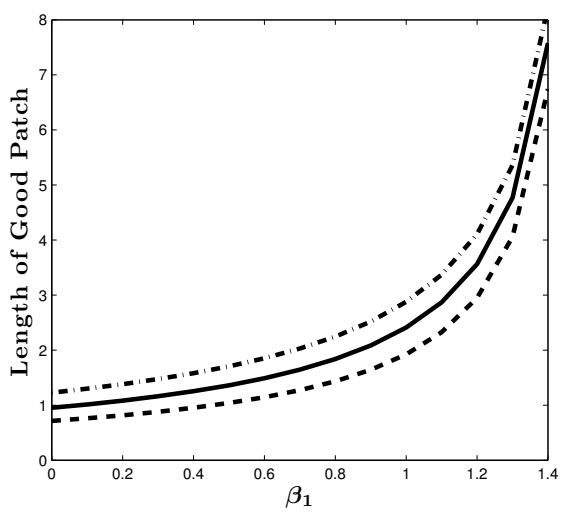

(b)

Figure 4.6: Critical size of good patches ((4.4.11)-(4.4.12)) with respect to mortality assuming the interface conditions are given by case $\mathrm{M}$ (dashed), $\mathrm{S}$ (solid), and $\mathrm{C}$ (dashed-dot) for (a) increasing values of $\beta_{2}, \beta_{1}=1$ and (b) increasing values of $\beta_{1}, \beta_{2}=1$. Fixed parameter values are: $\nu_{1}=1, \nu_{2}=2$, $\alpha_{1}=1, \alpha_{2}=1, l_{2}=2, r_{1}=2.5, r_{2}=0.6$, and $z=0$.

the population no longer persists, regardless of how slow/fast individuals move in good patches.

\subsection{Effect of Relevant Scales on the Critical Size of Good Patches}

In this section, we examine the dependence of the critical size of good patches on the scale of dispersal relative to the scale of landscape heterogeneity. Several studies suggested that population persistence is highly sensitive to the ratio of these scales (VanKirk, 1995; Fahrig, 2002; With, 2002; Dewhirst and Lutscher, 2009).

Following VanKirk (1995), we introduce dimensionless parameters to measure dispersal ability, habitat quality and habitat heterogeneity. Dispersal ability will be measured by relating the mean (directional) dispersal distance of $k$ to patch length; habitat quality measures the quality of good patches relative to bad ones; and habitat 
heterogeneity measures the dispersal ability of individuals relative to the quality of the patch.

Recall that the mean dispersal distance in patch type $i$ is given by

$$
\frac{1}{\mu_{i}}=\sqrt{\frac{\nu_{i}}{\alpha_{i}+\beta_{i}}}, i=1,2 .
$$

We introduce the effective domain length as

$$
\hat{l}_{i}=\frac{l}{2} \mu_{i}, \quad i=1,2
$$

where the factor $\frac{l}{2}$ appears due to the symmetry of the landscape as is done in (4.4.5). The effective domain length is the length of the domain relative to the dispersal ability of individuals in good and bad patches.

Scaling the spatial variable in $(4.4 .5)$ by $\hat{l}_{1}$ and $\hat{l}_{2}$, the eigenvalue problem on the dimensionless interval $[0,1]$ is given by

$$
\begin{gathered}
\frac{d^{2}}{d x^{2}} \phi(x)+\hat{l}_{1}^{2}\left[\omega \hat{r}_{1}-1\right] \phi(x)=0, x \in(0, \hat{p}) \\
\frac{d^{2}}{d x^{2}} \phi(x)-\hat{l}_{2}^{2}\left[1-\omega \hat{r}_{2}\right] \phi(x)=0, x \in(\hat{p}, 1),
\end{gathered}
$$

where $\hat{p}=\frac{l_{1}}{l}$ is the percentage of good habitat. Thus, low values of $\hat{p}$ correspond to high fragmentation levels and large loss of suitable habitat whereas large values of $\hat{p}$ correspond to low fragmentation levels and low loss of suitable habitat. The eigenvalue problem (4.5.3) can be further simplified by introducing parameters to measure habitat quality and heterogeneity of the landscape.

The quality of the habitat is expressed as the ratio of the quality of good patches relative to bad patches and is given by

$$
q=\sqrt[4]{\frac{\hat{r}_{1}-1}{1-\hat{r}_{2}}}
$$


Assuming $\hat{r}_{2}$ is fixed and less than unity, increasing values of $q$ corresponds to increasing values of $\hat{r}_{1}$, which indicates an increase in habitat quality (measured as fecundity). In the limit when $\beta_{2} \rightarrow \infty$, we have $\hat{r}_{2} \rightarrow 0$, so that $q$ only depends on the reproductive rate in good patches, $\hat{r}_{1}$.

To quantify habitat heterogeneity, we introduce

$$
H_{i}^{2}=\hat{l}_{i}^{2} \sqrt{\left(\hat{r}_{1}-1\right)\left(1-\hat{r}_{2}\right)}, i=1,2
$$

which measure the quality of patch $i$ relative to the dispersal ability of individuals in patch $i$. For example, as $H_{1} \rightarrow 0$, either $\hat{l}_{1}^{2} \approx 0$ or the difference in habitat quality is small. Thus individuals are highly mobile in patch type I and/or the difference between the quality of good and bad patches is small. For large values of $H_{1}$, either $\hat{l}_{1}^{2}$ is large or the difference in habitat quality is large. In this case, individuals are almost sedentary in good patches and/or the difference between the quality of good and bad patches is large.

Upon setting $\omega=1$ and simplifying the system of ODEs (4.5.3) using expressions (4.5.4) and (4.5.5), we obtain

$$
\begin{gathered}
\frac{d^{2}}{d x^{2}} \phi(x)+H_{1}^{2} q^{2} \phi(x)=0, x \in(0, \hat{p}) \\
\frac{d^{2}}{d x^{2}} \phi(x)-\frac{H_{2}^{2}}{q^{2}} \phi(x)=0, x \in(\hat{p}, 1) .
\end{gathered}
$$

The general solution of (4.5.6) is given by

$$
\phi(x)=\left\{\begin{array}{l}
A \cos \left(H_{1} q x\right)+B \sin \left(H_{1} q x\right), x \in(0, \hat{p}) \\
C \cosh \left(\frac{H_{2}}{q} x\right)+D \sinh \left(\frac{H_{2}}{q} x\right), x \in(\hat{p}, 1) .
\end{array}\right.
$$

To determine the persistence boundary, we assume that interface conditions are as in Case M, and obtain the following implicit equation for the minimum percentage 
of good habitat, $\hat{p}$, necessary for persistence

$$
\tan \left(H_{1} q \hat{p}\right)-\frac{\bar{z} H_{2}}{q^{2} H_{1}} \tanh \left(\frac{H_{2}}{q}(1-\hat{p})\right)=0 .
$$

The corresponding equation in Case $\mathrm{S}$ is given by

$$
\tan \left(H_{1} q \hat{p}\right)-\sqrt{\frac{\nu_{2}}{\nu_{1}}} \frac{\bar{z} H_{2}}{q^{2} H_{1}} \tanh \left(\frac{H_{2}}{q}(1-\hat{p})\right)=0
$$

and for Case C by

$$
\tan \left(H_{1} q \hat{p}\right)-\frac{\nu_{2}}{\nu_{1}} \frac{\bar{z} H_{2}}{q^{2} H_{1}} \tanh \left(\frac{H_{2}}{q}(1-\hat{p})\right)=0 .
$$

By assuming that $H_{1}, H_{2}$, and $q$ are independent of other model parameters, we reduce the number of parameters in (4.5.8) from ten to five. This reduction in parameter space allows us to study equation (4.5.8) to make some general conclusions about the influence of relevant scales on population persistence. Since (4.5.9) and (4.5.10) have an additional factor involving $\nu_{1} / \nu_{2}$, we only illustrate the dependence of $\hat{p}$ on the relevant scales for Case M.

In Figure 4.7(a), we plot solution curves to (4.5.8) in $\left(\hat{p}, H_{2}\right)$ parameter space assuming $H_{1}=0.5$ (solid curve), $H_{1}=1$ (dashed curve) and $H_{1}=2$ (dashed-dot curve). The region where the trivial solution is unstable is located below each curve. When individuals are highly mobile in good patches and/or the difference in habitat quality is small (small $H_{1}$ ), the population only persists for large $\hat{p}$ values and small $H_{2}$ values. In other words, the size of good patches must be large relative to the size of bad patches, and individuals must be highly mobile in bad patches. As the value of $H_{1}$ increases, so that individuals become more sedentary in good patches and/or the difference between the quality of good and bad patches increases, the population can persist on a landscape with increasing fragmentation levels (decreasing $\hat{p}$ ) and individuals in bad patches are more sedentary (increasing $H_{2}$ ). 


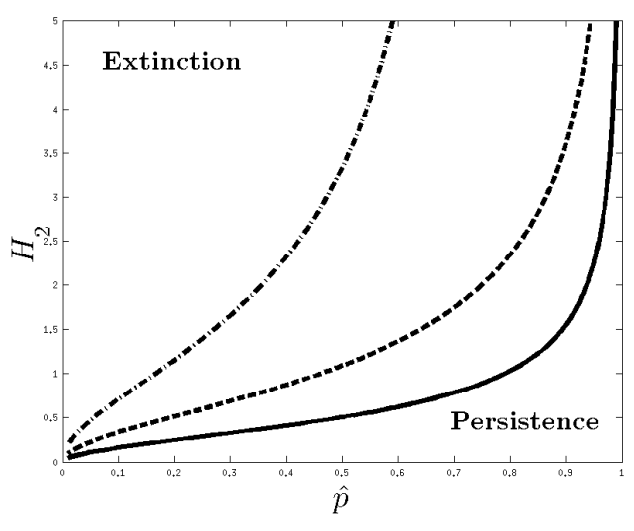

(a)

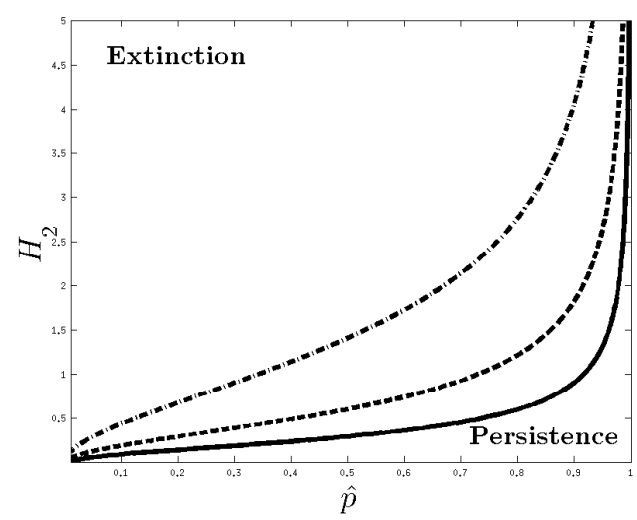

(b)

Figure 4.7: Solution curves to equation (4.5.8) in $\left(\hat{p}, H_{2}\right)$ parameter space, with interface conditions given by Case $\mathrm{M}$ assuming (a) $H_{1}=0.5$ (solid curve), $H_{1}=1$ (dashed curve) and $H_{1}=2$ (dashed-dot curve), $q=1$ and $z=0$, (b) $z=0$ (solid curve), $z=0.6$ (dashed curve) and $z=0.9$ (dasheddot curve), $q=1$ and $H_{1}=0.3$. The region where the trivial solution is unstable is located below each curve.

To illustrate the effects of movement bias at an interface, we fix $q$ and $H_{1}$, and plot solutions curves to equation (4.5.8) in $\left(\hat{p}, H_{2}\right)$ parameter space for different values of $z$ in Figure 4.7(b). The region where the trivial solution is unstable is located below each curve. Since individuals are highly mobile in good patches and/or the difference in habitat quality is low, movement bias does not have a significant effect on persistence unless the bias, towards good patches, is strong. If fragmentation level and loss of suitable habitat are low, a strong movement bias can result in population persistence. However, if the opposite is true, movement bias has little effect on persistence.

To conclude this section, we note that, by plotting solution curves of (4.5.8) in $(q, \hat{p})$ space, we can relate our results to those of VanKirk (1995). Note that $\hat{p}$ and $q$ correspond to $R$ and $b$ in VanKirk (1995), respectively. In Figure 4.8, we plot solution curves of (4.5.8), assuming $H_{1}$ and $H_{2}$ are fixed. When $H_{1}$ and $H_{2}$ are both small, corresponding to mobile organisms in both patch types, the fragmentation level 


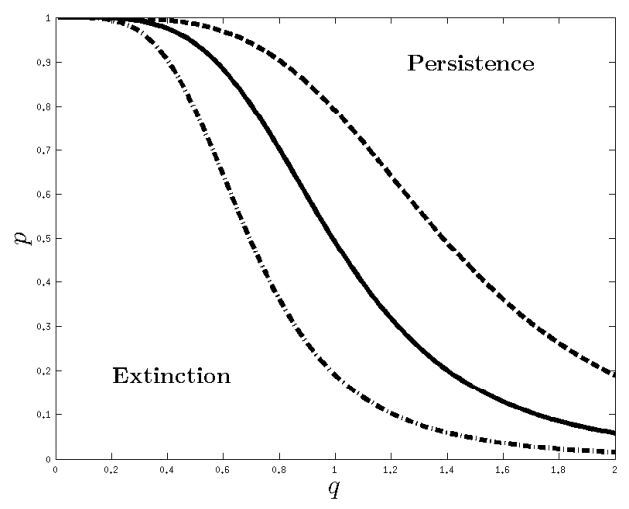

Figure 4.8: Solution curves of $(4.5 .8)$ in $(q, \hat{p})$ parameter space with no movement bias (i.e., $z=0$ ). The solid curve corresponds to $H_{1}=H_{2}$, the dashed curve corresponds to $H_{2}>H_{1}$ and the dashed-dot curve corresponds to $H_{1}>H_{2}$. The region where the trivial solution is stable is located below each curve.

must be low $(\hat{p} \approx 1)$ or the quality of the landscape high ( $q$ large) in order for the population to persist, agreeing with the results of VanKirk (1995) for constant $H$. Note that constant $H$ occurs when dispersal is not patch dependent so that $H_{1}=H_{2}$.

When individuals tend to spend more time in bad patches (dashed curve), we observe that the size of good patches relative to bad patches must be large $(\hat{p} \approx 1)$ irrespective of the value of $q$. In other words, if $H_{2}>H_{1}$, the fragmentation level of the landscape must be low. On the other hand, if individuals tend to spend more time in good patches (dashed-dot curve), the fragmentation level does not significantly affect persistence conditions, and good patches may be separated by larger distances. The latter conclusion agrees with the results of VanKirk when individuals were sedentary in his model. Therefore, for this parameter set, we see that as, $H_{1}$ becomes large relative to $\mathrm{H}_{2}$, persistence conditions agree with those obtained under the case of spatially homogeneous dispersal functions. 


\section{Chapter 5}

\section{Analysis of Integrodifference \\ Equations: Traveling Periodic \\ Waves and the Spreading Speed}

When a population can persist in some landscape, one can expect that a locally introduced population will spread spatially, at least as long as there is no Allee effect. The speed of this spatial spread is a quantity of great ecological interest and importance. Likewise, the study of this spreading speed has generated great interest in mathematical analysis.

For an infinite homogeneous landscape, Weinberger (1982) proved the existence of a spreading speed (see Definition 1.3.5) under some conditions on $k$ and $f$ (see Introduction). He also proved that linear determinacy holds; i.e., the spreading speed for the nonlinear equation equals the spreading speed of the linearized equation at zero, which in turn equals the minimal speed of a traveling wave.

Later, he generalized these results to periodically varying landscapes (Weinberger, 2002). This insight justifies the approach by Shigesada et al. (1986) for reaction-diffusion equations and Robbins (2004), as well as Kawasaki and Shigesada 
(2007), for integrodifference equations. Those authors derived a "dispersion relation" between the speed and the steepness of a traveling periodic wave in periodic landscapes with piecewise constant coefficient functions.

The main results in this chapter are the derivation of the dispersion relation (see Section 5.2) and the discussion and interpretation of the resulting minimal speed (see Section 5.3). At first, however, we briefly discuss why the results of Weinberger (2002) do not apply to our system and what the difficulties are to generalize his theory to our case (Section 5.1). Numerical simulations justify our approach by showing a close correspondence between the spread rate obtained from the dispersion relation and from numerical simulations.

\subsection{Traveling Periodic Waves}

Weinberger (2002) considered equation (1.2.1) on a periodic landscape in the following sense:

$$
f(\cdot, x)=f(\cdot, x+l) ; \quad k(x+l, y+l)=k(x, y),
$$

for some $l>0$ and all $x, y \in \mathbb{R}$.

Under certain conditions on the operator $Q$, he proved the existence of a spreading speed. More precisely, he worked on the following set of continuous functions

$$
\mathcal{M}=\left\{N(x): N \text { is continuous on } \mathbb{R}, 0 \leq N(x) \leq \pi_{1}(x)\right\}
$$

where $\pi_{1}$ is a positive fixed point of (1.2.1), and required that the following assumptions hold for $Q$ :

\section{Hypotheses 2.1 in Weinberger (2002)}

i. The domain, $\mathcal{H}$, is a closed subset of $\mathbb{R}$.

ii. $Q$ is order-preserving in the sense that if $N(x) \leq V(x)$ on $\mathcal{H}$, then $Q[N](x) \leq$ 
$Q[V](x)$. That is, an increase throughout $\mathcal{H}$ in the population $N_{t}$ at time $t$ increases the population $N_{t+1}=Q\left[N_{t}\right]$ throughout $\mathcal{H}$ at the next time step.

iii. There is a closed d-dimensional lattice $L$ such that $\mathcal{H}$ is invariant under translation by any element of $L$, and $Q$ is periodic with respect to $L$ in the sense that $Q\left[T_{a}[N]\right]=T_{a}[Q[N]]$ holds for all $N \in \mathcal{M}$ and $a \in L$, where $T$ is the translation operator defined as $T_{a}[N](x):=N(x-a)$. Moreover, there is a bounded subset $P$ of $\mathcal{H}$ such that every $x \in \mathcal{H}$ has a unique representation of the form $x=z+p$ with $z$ in $L$ and $p$ in $P$.

iv. $Q[0]=0$, and there are L-periodic equilibria $\pi_{0}(x)$ and $\pi_{1}(x)$ such that $0 \leq$ $\pi_{0}<\pi_{1}, Q\left[\pi_{0}\right]=\pi_{0}$ and $Q\left[\pi_{1}\right]=\pi_{1}$. Moreover if $\pi_{0} \leq N_{0} \leq \pi_{1}, N_{0}$ is periodic with respect to $L$, and $N_{0} \not \equiv \pi_{0}$, then the solution $N_{t}$ of the recursion (1.2.1), which is again periodic with respect to $L$, converges to $\pi_{1}$ as $t \rightarrow \infty$ uniformly on $\mathcal{H}$. (That is, $\pi_{0}$ is unstable and $\pi_{1}$ is stable.) In addition, any L-periodic equilibrium $\pi$ other than $\pi_{1}$ which satisfies the inequalities $0 \leq \pi \leq \pi_{1}$ also satisfies $\pi \leq \pi_{0}$.

v. $Q$ is continuous in the sense that if the sequence $\left\{N_{j}\right\}_{j \in \mathbb{N}} \in \mathcal{M}$ converges to $N \in \mathcal{M}$ uniformly on every bounded subset of $\mathcal{H}$, then $\left\{Q\left[N_{j}\right]\right\}_{j \in \mathbb{N}}$ converges to $Q[N]$ uniformly on every bounded subset of $\mathcal{H}$. That is, a change in $N$ far from the point $x$ has very little effect on the value of $Q[N]$ at $x$.

vi. Every sequence $\left\{N_{j}\right\}_{j \in \mathbb{N}}$ of functions in $\mathcal{M}$ contains a subsequence $\left\{N_{j_{m}}\right\}_{m \in \mathbb{N}}$ such that $\left\{Q\left[N_{j_{m}}\right]\right\}_{m \in \mathbb{N}}$ converges to some function, uniformly on every bounded set.

Remark: The definition of $\mathcal{H}$ in (1) above allows for the treatment of continuous and discrete habitats as considered by Weinberger. For our purposes, it suffices to take $\mathcal{H}=\Omega=\mathbb{R}, L=\{(m l,(m+1) l: m \in \mathbb{Z}\}$, and $P=\{(0, l)\}$. Then, $\mathcal{H}$ is clearly invariant under translation by any $\tilde{z} \in L$, and each $x$ in $\mathcal{H}$ has a unique representation 
of the form $x=\tilde{z}+p$ with $\tilde{z} \in L$ and $p \in P$. Additionally, note that in our notation $\pi_{0}=0$, and $\pi_{1}=N^{*}$.

Weinberger then proved the following result.

Theorem 5.1.1 (The Spreading Speed) Let $\left\{N_{t}\right\}$ be a solution of the recursion (1.2.1) and assume that the initial population, $N_{0}(x)$, is positive and has compact support. Then, there exists a number $c^{*}$ such that solutions of the recursion (1.2.1) have the following spreading properties:

1. If $N_{0}(x) \geq 0, \inf \left\{\pi_{1}(x)-N_{0}(x)\right\}>0, N_{0}(x)=0$ for $|x| \geq L$, and if $c^{*}<\infty$, then for every $c>c^{*}$

$$
\limsup _{t \rightarrow \infty}\left(\sup _{|x| \geq c t}\left[N_{t}(x)-\pi_{0}(x)\right]\right) \leq 0
$$

2. If $0 \leq N_{0} \leq \pi_{1}$ and there is a constant $C$ such that $\inf _{|x| \leq C}\left\{N_{0}(x)-\pi_{0}(x)\right\}>0$ then for every $c<c^{*}$

$$
\lim _{t \rightarrow \infty}\left(\sup _{|x| \leq c t}\left[\pi_{1}(x)-N_{t}(x)\right]\right)=0 .
$$

Remark: Weinberger proved the existence of the spreading speed and corresponding traveling wave solution under the assumption that $\pi_{0}=0$. In this case, he also showed that $c^{*}$ corresponds to the speed and minimal speed of a traveling periodic wave (TPW). If $\pi_{0} \neq 0$, he was unable to show the existence of a traveling wave solution and corresponding spreading speed.

In general, there is no simple way to calculate $c^{*}$. A notable and important exception is the case that the speed is linearly determined; i.e., the spreading speed is given by the spreading speed of the linearized equation. Under the assumption that the spreading speed is also the minimal speed for a TPW, one may look for solutions 
of the recursion (1.3.1) of the form

$$
N_{t}(x)=e^{-s(x-c t)} \theta(x)
$$

where $s$ is the shape parameter of the wave and $\theta$ is an $l$-periodic function. Substituting this ansatz into (1.3.1) we obtain

$$
e^{s c} \theta(x)=B_{s}[\theta(y)](x)
$$

where $B_{s}[\theta(y)](x)=e^{s x} B\left[e^{-s y} \theta(y)\right](x)$. Thus, from (5.1.4), we see that $e^{s c}$ is an eigenvalue of the operator $B_{s}$ with a corresponding eigenfunction given by $\theta(x)$. The relationship between $c$ and the eigenvalue is $e^{s c}=\lambda(s)$ and the explicit formula for the spreading speed is

$$
c^{*}=\inf _{s>0}\{(1 / s) \ln (\lambda(s))\} .
$$

The following result of Weinberger (2002) shows that the spreading speed is linearly determined if $B$ is an $L$-periodic, strongly order-preserving operator that satisfies the six hypotheses listed above and possesses a dominant eigenvalue.

Theorem 5.1.2 (Linear Determinacy in Periodic Landscapes) Suppose that the linearization of $Q$ at $N=0$ is given by an operator $B$ such that

1. $Q[N] \geq(1-\varrho) B[N]$ for every $N$ such that $0 \leq N \leq \eta$ and every positive number $\varrho$

2. $Q[N] \leq B[N]$ for all $N$ with $0 \leq N \leq N^{*}$;

3. $B$ is L-periodic and strongly-order preserving, and $B\left[e^{s|x|}\right]$ is defined for all $s$;

4. there is an L-periodic function $r$ such that $B[N]>r$, and the truncated operator

$$
Q^{[B, r]}[N]:=\min \{B[N], r\}
$$

satisfies Hypotheses 2.1. 
Then, if the linear opeartor $B$ possesses a dominant eigenvalue, $\lambda$, the spreading speed is linearly determinante and given by (5.1.5).

In the following, we discuss which parts of Weinberger's theory carry over to our situation and which require novel analytical techniques. We provide numerical evidence that, nonetheless, the formula for calculating the spread rate from the linearized problem applies.

In Chapter 4, we derived conditions under which the trivial solution of (1.2.1) is unstable. We now show that $Q$ in (1.2.1) possesses a positive, periodic steady state that is globally stable when persistence conditions (e.g. (4.4.11)) are satisfied. We assume that parameter functions are $l$-periodic, piecewise constant where $\nu$ is given by (4.2.1) and $\alpha, \beta$, and $\hat{r}$ are analogously defined. Furthermore, it is assumed that $f(\cdot, x)$ is $l$-periodic in $x$. In this case, the dispersal kernel and growth function satisfy (5.1.1).

We employ the results of Lemma 4.2.2 and work on the set of $l$-periodic, nonnegative, bounded functions on $\mathbb{R}$. By restricting $l$-periodic functions on $\mathbb{R}$ to one period; e.g., $\Omega=[0, l]$ and noting the operator $Q$ in (1.2.1) is monotone and concave since $f$ is given by the Beverton-Holt function (1.2.5), one can show the existence, uniqueness, and global stability of a positive fixed point, $N^{*}(x)$. The result follows from several theorems of Krasnosel'skii (1964a) with details presented in Appendix E. The majority of this analysis follows from Appendix B.6 in VanKirk (1995). The fact that $N^{*}(x)$ is also an $l$-periodic fixed point; i.e., $N^{*}(x)=N^{*}(x+l)$, follows from Lemma 4.2.3.

Having conditions under which the population can persist and existence of a positive, periodic steady state, we numerically investigate the qualitative behavior of recursion (1.2.1) assuming the dispersal kernel is discontinuous. As an example, we iterated recursion (1.2.1) over twenty generations assuming the dispersal kernel is obtained from interface conditions given by Case M (2.4.8). Parameter values are 
chosen such that (4.4.11) is satisfied so that the population persists. Observe from Figure 5.1(a) that the spatial profile of the population evolves into a discontinuous traveling periodic wave with population densities larger in good patches and smaller in bad patches. The discontinuity in the spatial profile occurs at interfaces between the two patch types where there is a jump discontinuity in the kernel; see Figure 5.1(b). Figure 5.1(c) shows that the front of the population advances at a constant rate so that the average frontal speed given by (1.3.15) is constant.

Based on simulating equation (1.2.1) over a wide range of parameter space, we make the following observations. When persistence conditions; e.g., (4.4.11) are not satisfied, the invasion fails. However, when persistence conditions are satisfied and the dispersal kernel is discontinuous (e.g. $\bar{z} \gamma_{1} \neq 1$ in (2.4.8)), the spatial profile of the invasion evolves into a discontinuous traveling periodic wave that moves with constant speed. We conjecture that this is a robust solution of equation (1.2.1) and leave the mathematical details as future work.

There are two difficulties with applying the theory of Weinberger, which assumes $Q$ is a compact operator that maps bounded continuous functions into itself, and leaves the set $\mathcal{M}$ invariant, to our system. First, as shown in Figure 5.1, solutions to equation (1.2.1) equipped with a (discontinuous) kernel derived in Chapters 2 and 3 are not continuous.

In a homogeneous landscape, the way to construct an invariant set $\mathcal{M}$ is to consider the non-spatial dynamics. For example, if $f$ is given by the non-dimensionalized Beverton-Holt function (1.2.5), then $\mathcal{M}=\{0 \leq N \leq 1\}$. For a kernel satisfying the assumptions in Chapter 1, the operator $Q$ in (1.2.1) leaves the set $\mathcal{M}$ invariant. If $Q$ is equipped with a discontinuous kernel derived in Chapters 2 and 3, this set $\mathcal{M}$ is not necessarily invariant for the following reasons.

The combination of patch-dependent movement and settling rates, potentially including patch preference, in the movement model has the effect that the kernel $k$ can in some sense concentrate individuals in a certain area (e.g., a good patch). This 


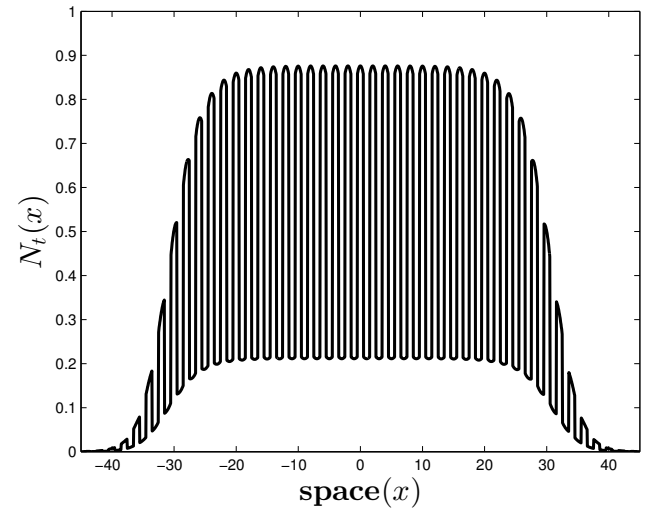

(a)

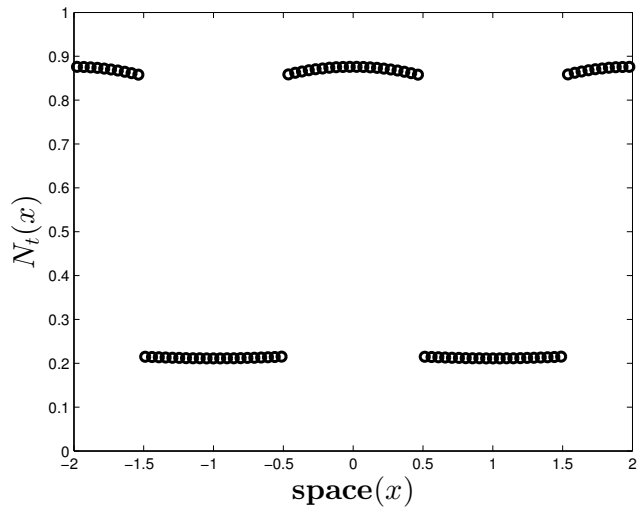

(b)

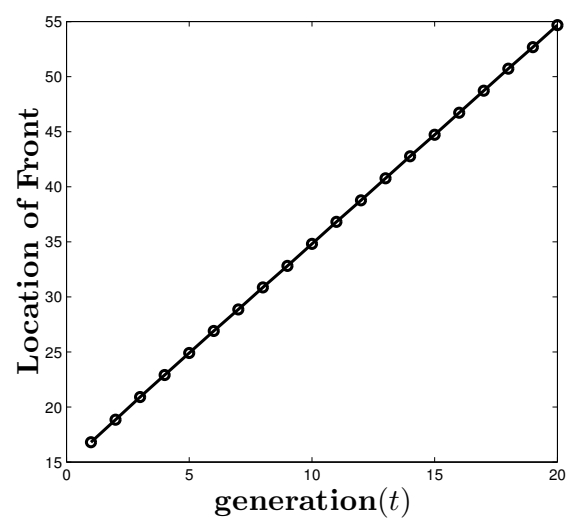

(c)

Figure 5.1: (a) Discontinuous traveling periodic wave obtained by numerically iterating (1.2.1) for twenty generations with $N_{0}(x) \equiv N_{0} \delta(x)$. The dispersal kernel was obtained from Case $\mathrm{M}$ interface conditions. (b) Blow-up of image in (a) showing discontinuities occur at interfaces between two patch types. (c) Location of the wave front as a function of generation time showing the population advances at a constant rate. Parameter values are chosen such that (4.4.11) is satisfied and given by: $l_{1}=l_{2}=1, \alpha_{1}=1, \alpha_{2}=1$, $\nu_{1}=0.5, \nu_{2}=1.5, \beta_{1}=\beta_{2}=0, r_{1}=3, r_{2}=0.7$, and $z=0$. 


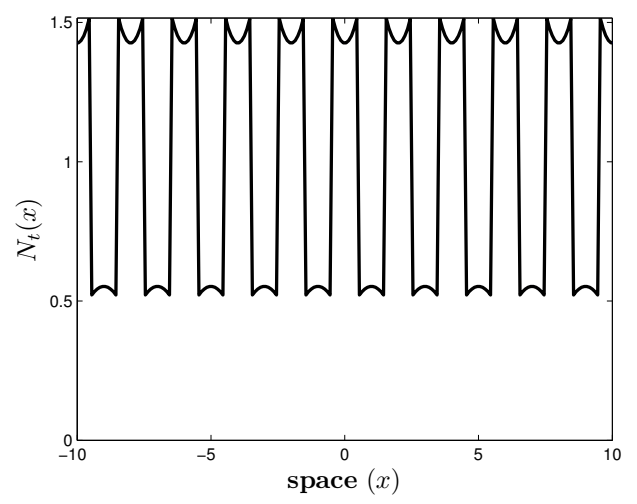

Figure 5.2: Numerical solution of (1.2.1) after one generation with $f$ given by the function that is identically one. Obtained densities larger than unity show the non-existence of an invariant subset. The dispersal kernel was obtained from Case $M$ interface conditions. Parameter values are chosen such that (4.4.11) is satisfied and given by: $l_{1}=l_{2}=1, \alpha_{1}=1, \alpha_{2}=1, \nu_{1}=0.5$, $\nu_{2}=1.5, \beta_{1}=\beta_{2}=0$, and $z=0$.

phenomenon may prevent the existence of an invariant domain for the next-generation operator. Consider, for example, the case that the growth function is spatially independent, given by the scaled Beverton-Holt function (1.2.5). Then $f(1, x)=1$ for all $x$. However, under certain conditions on movement parameters, we may have $Q[1](x)>1$ for some $x$ (see Figure 5.2). In particular, the set of all functions with values between 0 and 1 is not invariant here.

\subsection{Dispersion Relation for a Traveling Periodic Wave}

The results of the above section suggest that, if the spreading speed of (1.2.1) exists it is linearly determinante, we can consider the linearization of (1.2.1) given by

$$
N_{t+1}(x)=\int_{-\infty}^{\infty} r_{0}(y) k(x, y) N_{t}(y) d y
$$


to derive the dispersion relation for $c$.

Following Shigesada et al. (1986), we look for a traveling periodic wave of the form

$$
N_{t}(x)=e^{-s(x-c t)} \theta(x)
$$

where $\theta(x)=\theta(x+l)$ is a periodic function. Substituting ansatz (5.2.2) into (5.2.1), we obtain

$$
e^{-s(x-c)} \theta(x)=\int_{-\infty}^{\infty} r_{0}(y) e^{-s y} \theta(y) k(x, y) d y
$$

Integral equation (5.2.3) may once again be transformed into an ODE by applying the definition of $k$ in (2.2.6) to (5.2.3) to obtain

$$
\frac{d^{2}}{d x^{2}}\left(\frac{\nu(x)}{\alpha(x)} \theta(x) \exp (-s x)\right)+\hat{s}(x) \theta(x) \exp (-s x)(\exp (-s c) \hat{r}(x)-1)=0
$$

where $\hat{s}(x)=\frac{\alpha(x)+\beta(x)}{\alpha(x)}$. Substituting $\Psi(x)=\frac{\nu(x)}{\alpha(x)} \theta(x) \exp (-s x)$ simplifies this ODE to

$$
\Psi^{\prime \prime}(x)+m(x) \Psi(x)(\exp (-s c) \hat{r}(x)-1)=0
$$

where $m(x)=\frac{\alpha(x)+\beta(x)}{\nu(x)}$.

To derive the dispersion relation, we need to specify interface conditions. We give the derivation for Case $\mathrm{M}$ only; the other cases are similar. It turns out that the notation simplifies by choosing patches of type 1 (good patches) to occur for $x \in\left(0, l_{1}\right)+l \mathbb{Z}$ and patches of type 2 (bad patches) for $x \in\left(-l_{2}, 0\right)+l \mathbb{Z}$.

Applying the first interface condition in (2.4.8) to integral equation (5.2.3) for $\theta$ 
and taking into consideration that $\theta(x)$ is $l$-periodic, we obtain

$$
\begin{aligned}
\lim _{x \rightarrow l_{1}^{-}} e^{-s(x-c)} \theta(x) & =\lim _{x \rightarrow l_{1}^{-}} \int_{-\infty}^{\infty} r_{0}(y) \exp (-s y) \theta(y) k(x, y) d y \\
& =\int_{-\infty}^{\infty} r_{0}(y) \exp (-s y) \theta(y) \lim _{x \rightarrow l_{1}^{-}} k(x, y) d y \\
& =\frac{(1+z) \nu_{2} \alpha_{1}}{(1-z) \nu_{1} \alpha_{2}} \int_{-\infty}^{\infty} r_{0}(y) \exp (-s y) \theta(y) \lim _{x \rightarrow l_{1}^{+}} k(x, y) d y \\
& =\frac{(1+z) \nu_{2} \alpha_{1}}{(1-z) \nu_{1} \alpha_{2}} \lim _{x \rightarrow l_{1}^{+}} e^{-s(x-c)} \theta(x) \\
& =\frac{(1+z) \nu_{2} \alpha_{1}}{(1-z) \nu_{1} \alpha_{2}} e^{-s\left(l_{1}-c\right)} \lim _{x \rightarrow-l_{2}^{+}} \theta(x),
\end{aligned}
$$

and, therefore, one interface condition for $\theta$ is

$$
(1-z) \nu_{1} \alpha_{2} \theta\left(l_{1}^{-}\right)=(1+z) \nu_{2} \alpha_{1} \theta\left(-l_{2}^{+}\right)
$$

where the notation \pm is short for the right and left-hand limits, respectively. Similarly, we derive interface conditions for the derivatives as

$$
\nu_{1} \alpha_{2} \theta^{\prime}\left(l_{1}^{-}\right)=\nu_{2} \alpha_{1} \theta^{\prime}\left(-l_{2}^{+}\right)
$$

Likewise, the interface conditions at $x=0$ are given by

$$
\begin{aligned}
(1+z) \nu_{2} \alpha_{1} \theta\left(0^{-}\right) & =(1-z) \nu_{1} \alpha_{2} \theta\left(0^{+}\right) \\
\nu_{2} \alpha_{1} \theta^{\prime}\left(0^{-}\right) & =\nu_{1} \alpha_{2} \theta^{\prime}\left(0^{+}\right) .
\end{aligned}
$$

Using the relation between $\theta$ and $\Psi$, we obtain the following interface conditions 
for $\Psi$

$$
\begin{aligned}
(1-z) \Psi\left(l_{1}^{-}\right) & =(1+z) e^{-s l} \Psi\left(-l_{2}^{+}\right) \\
\Psi^{\prime}\left(l_{1}^{-}\right) & =e^{-s l} \Psi^{\prime}\left(-l_{2}^{+}\right) \\
(1+z) \Psi\left(0^{-}\right) & =(1-z) \Psi\left(0^{+}\right) \\
\Psi^{\prime}\left(0^{-}\right) & =\Psi^{\prime}\left(0^{+}\right) .
\end{aligned}
$$

Applying the definitions of $\alpha, \beta, \hat{r}$ and $\nu$ (4.2.1) to (5.2.5), we obtain the following system of ODEs for $\Psi$

$$
\begin{aligned}
& \Psi^{\prime \prime}(x)+\mu_{1}^{2}\left(e^{-s c} \hat{r}_{1}-1\right) \Psi(x)=0, \quad x \in\left(0, l_{1}\right)+l \mathbb{Z} \\
& \Psi^{\prime \prime}(x)-\mu_{2}^{2}\left(e^{-s c} \hat{r}_{2}-1\right) \Psi(x)=0, \quad x \in\left(-l_{2}, 0\right)+l \mathbb{Z} .
\end{aligned}
$$

Since $\Psi(x+l)=\Psi(x) \exp (-s l)$ holds, we may restrict our analysis to one period. The solution to the above system of ODEs (5.2.9) is given by

$$
\begin{aligned}
& \Psi_{1}(x)=A_{1} \cosh \left(q_{1} x\right)+B_{1} \sinh \left(q_{1} x\right), \quad x \in\left(0, l_{1}\right) \\
& \Psi_{2}(x)=A_{2} \cosh \left(q_{2} x\right)+B_{2} \sinh \left(q_{2} x\right), \quad x \in\left(-l_{2}, 0\right),
\end{aligned}
$$

where $q_{i}=\sqrt{\mu_{i}^{2}\left(e^{-s c} \hat{r}_{i}-1\right)}, i=1,2$. The interface conditions (5.2.8) lead to the following system of linear equations for $A_{1}, A_{2}, B_{1}$ and $B_{2}$ :

$$
\begin{aligned}
& A_{1} \bar{z}-A_{2}=0 \\
& B_{1} q_{1}-B_{2} q_{2}=0 \\
& A_{1} \cosh \left(q_{1} l_{1}\right)+B_{1} \sinh \left(q_{1} l_{1}\right)-\frac{e^{s l}}{\bar{z}}\left(A_{2} \cosh \left(q_{2} l_{2}\right)-B_{2} \sinh \left(q_{2} l_{2}\right)\right)=0 \\
& q_{1}\left(A_{1} \sinh \left(q_{1} l_{1}\right)+B_{1} \cosh \left(q_{1} l_{1}\right)\right)+q_{2} e^{s l}\left(A_{2} \sinh \left(q_{2} l_{2}\right)-B_{2} \cosh \left(q_{2} l_{2}\right)\right)=0 .
\end{aligned}
$$


The above system (5.2.11) may be written as the matrix equation

$$
\mathbf{A} \varphi_{2}=\mathbf{0},
$$

where $\varphi_{\mathbf{2}}=\left[A_{1}, B_{1}, A_{2}, B_{2}\right]^{T}, \mathbf{0}=[0,0,0,0]^{T}$, and $\mathbf{A}$ is the coefficient matrix. Therefore, a necessary condition for $\Psi$ to be non-zero is $\operatorname{det}(\mathbf{A})=0$. This constraint leads to the dispersion relation that is an implicit equation between the minimum speed of the wave, $c$, and its shape parameter given by

$$
\kappa_{m} \sinh \left(q_{1} l_{1}\right) \sinh \left(q_{2} l_{2}\right)+\cosh \left(q_{1} l_{1}\right) \cosh \left(q_{2} l_{2}\right)-\cosh (s l)=0
$$

where $\kappa_{m}=\frac{q_{1}^{2}+\left(q_{2} \bar{z}\right)^{2}}{2 \bar{z} q_{1} q_{2}}$.

Following the same steps as above with different interface conditions, we find the dispersion relation for Case $\mathrm{S}$ as

$$
\kappa_{s} \sinh \left(q_{1} l_{1}\right) \sinh \left(q_{2} l_{2}\right)+\cosh \left(q_{1} l_{1}\right) \cosh \left(q_{2} l_{2}\right)-\cosh (s l)=0,
$$

where $\kappa_{s}=\frac{\nu_{1} q_{1}^{2}+\nu_{2}\left(q_{2} \bar{z}\right)^{2}}{2 \bar{z} q_{1} q_{2} \sqrt{\nu_{1} \nu_{2}}}$, and for Case C as

$$
\kappa_{c} \sinh \left(q_{1} l_{1}\right) \sinh \left(q_{2} l_{2}\right)+\cosh \left(q_{1} l_{1}\right) \cosh \left(q_{2} l_{2}\right)-\cosh (s l)=0,
$$

where $\kappa_{c}=\frac{\left(\nu_{1} q_{1}\right)^{2}+\left(\nu_{2} q_{2}\right)^{2}}{2 q_{1} q_{2} \nu_{1} \nu_{2}}$

\subsection{Analysis of Dispersion Relation}

In a homogeneous landscape, the dispersion relation for the speed of a traveling wave is given by $c=\frac{1}{s} \ln \left(f^{\prime}(0) M(s)\right)$; see equation (1.3.13). Assuming $k$ is given by the Laplace kernel; e.g., equation (2.2.8) with $\beta=0$ and $f$ is given by the Beverton-Holt 
function (1.2.5), the dispersion relation is given by

$$
c=\frac{1}{s} \ln \left(r_{0} \frac{1}{1-\frac{\nu}{\alpha} s^{2}}\right) .
$$

Equation (5.3.1) can be obtained from any one of the three dispersion relations derived above. For example, consider the dispersion relation given by equation (5.2.12) with $l_{1}=l, \alpha_{1}=\alpha, \beta_{1}=0$, and $z=0$. Substituting these values into Dispersion relation (5.2.12), we obtain

$$
\cosh \left(q_{1} l\right)-\cosh (s l)=0
$$

which is satisfied if

$$
\sqrt{\frac{\alpha}{\nu}\left(e^{-s c} r_{0}-1\right)}=s
$$

Solving the above equation for $c$, one obtains the dispersion relation for the Laplace kernel given by (5.3.1).

By applying (5.1.5) to any of the dispersion relations derived above, we should obtain a candidate for the minimal speed of the wave. To check this, we calculated speeds, over a wide range of parameter space, from the dispersion relation for Case $\mathrm{M}$ and compared obtained values with the average frontal speed (1.3.15) obtained numerically. Figure 5.3(a) shows spreading speeds obtained from dispersion relation (5.2.12) (solid curve) and from numerical simulations (open circles) as a function of the reproductive rate in good patches, $r_{1}$. Similarly, Figure 5.3(b) shows obtained spreading speeds as a function of motility in good patches. Observe from both figures that the dispersion relation gives a very nice fit to numerically obtained speeds, and this observation holds over a wide range of parameter space.

Based on the above results, we conjecture that the spreading speed exists and is linearly determined. We now study how each of the dispersion relations depend on model parameters. In Figure 5.4, we plot the spreading speed for increasing values of $\nu_{2}$ and three different values of $l_{2}$, for Cases M, S, and C. The points where $c$ equals 


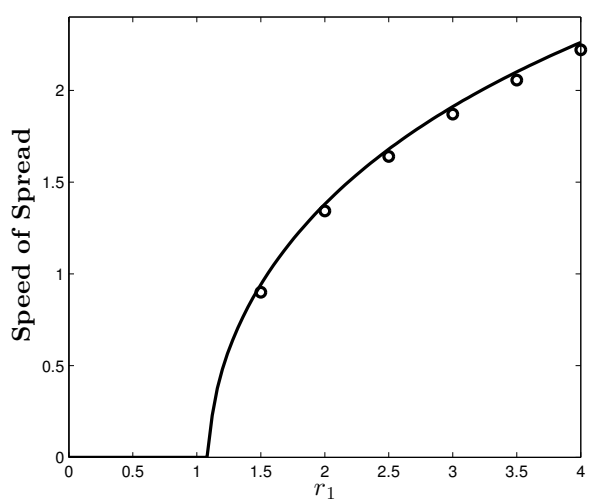

(a)

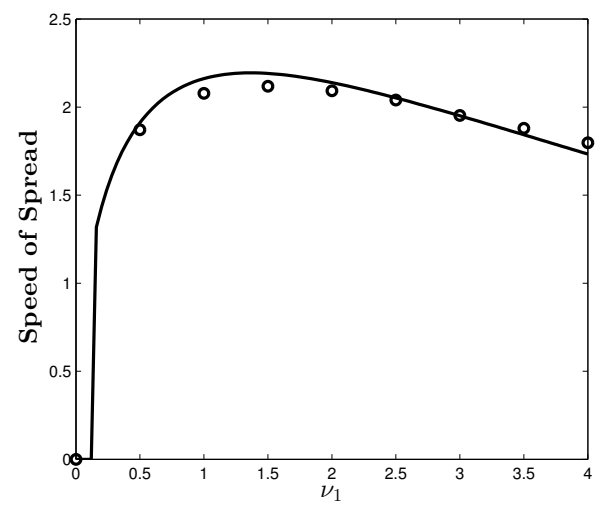

(b)

Figure 5.3: (a) Speed of spread calculated from dispersion relation (5.2.12) (solid curve) and from numerical simulations (closed circles) as a function of the reproductive rate in good patches, $r_{1}$ with $\nu_{2}=1.5$. (b) Speed of spread calculated from dispersion relation (5.2.12) (solid curve) and from numerical simulations (open circles) as a function of the motility in good patches, $\nu_{1}$ with $r_{1}=3$. Fixed parameter values are: $l_{1}=l_{2}=1, \alpha_{1}=1, \alpha_{2}=1$, $\beta_{1}=\beta_{2}=0, \nu_{2}=1.5, r_{2}=0.7$, and $z=0$. 
zero correspond to the persistence conditions shown in Figure 4.4. The spreading speed is an increasing function of $\nu_{2}$ for cases $\mathrm{M}$ and $\mathrm{S}$ with larger speeds obtained from Case M. Somewhat surprisingly, larger bad patches can lead to higher spreading speeds if motility in bad patches is high. For sufficiently small bad patches, the spread rate is an increasing function of $\nu_{2}$ for Case C; see Figure 5.4(c). However, if bad patches are large enough, the spreading speed is a nonmonotone function of $\nu_{2}$. The latter results agree with other authors (Shigesada et al., 1986; Maciel and Lutscher, 2013) as well as with our persistence conditions that show a population will always persist if $l_{2}$ is below a threshold value, regardless of the value of $\nu_{2}$ (plot not shown). The biological explanation of this phenomenon is that an increased value of $\nu_{2}$ corresponds to a larger probability of individuals to enter bad habitat and therefore decrease the chance of the population to persist.

The qualitative behavior of the spreading speed with respect to $\nu_{1}$ is shown in Figure 5.5, and the points where $c$ equals zero correspond to the persistence conditions shown in Figure 4.4. Regardless of the size of bad patches, the spreading speed is a hump-shaped function of individual movement in good patches for Cases $\mathrm{M}$ and $\mathrm{S}$. As $\nu_{1}$ approaches zero, individuals move very slowly, and the spreading speed decays to zero. If individuals move very fast in good patches, they reduce residence time in good patches. Persistence conditions are no longer satisfied and the spreading speed approaches zero. If bad patches are sufficiently small, the spreading speed is a monotone increasing function of $\nu_{1}$ for Case C. However, as $l_{2}$ increases, the spreading speed is a hump-shaped function of $\nu_{1}$ and eventually decreases to zero. Even though individuals have a higher probability of moving into a good patch, a sufficient number reduce their residence time in good patches and move to a bad patch. As the size of bad patches increases, individuals become trapped there and the population no longer persists.

The spreading speed is also a hump-shaped function of movement bias as shown in Figure 5.6. Increasing preference for good patches can increase the spread rate. 


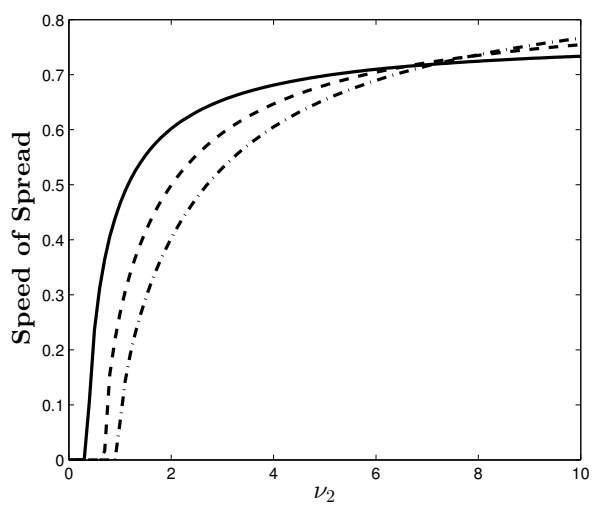

(a)

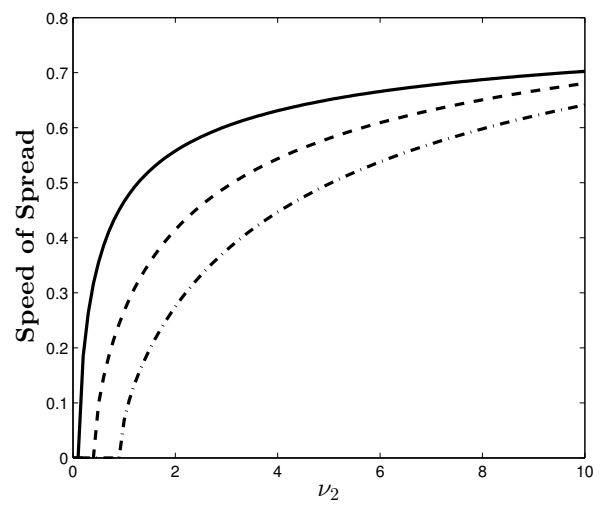

(b)

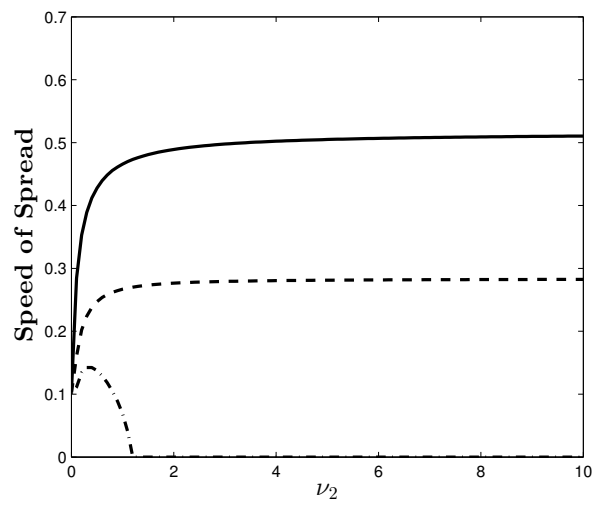

(c)

Figure 5.4: Spreading speed as a function of movement rate in bad patches for $l_{2}=0.5$ (solid), $l_{2}=1$ (dashed), $l_{2}=1.5$ (dashed-dot) assuming interface conditions are given by (a) Case M, (b) Case S, (c) Case C. Fixed parameter values are: $\alpha_{1}=1, \alpha_{2}=1, \nu_{1}=1, \beta_{1}=1, \beta_{2}=1, l_{1}=2.5, r_{1}=2.5$, $r_{2}=0.6$, and $z=0$. 


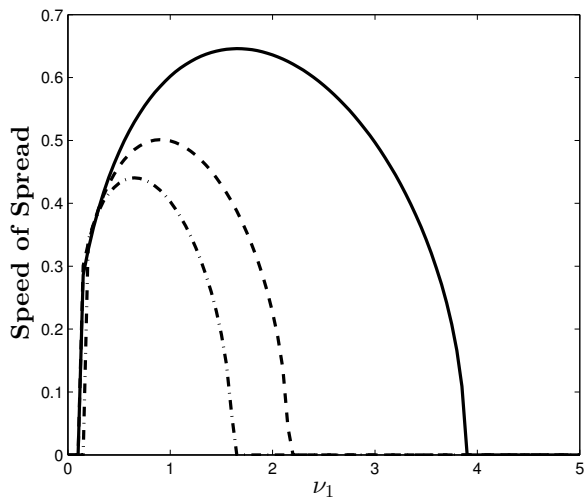

(a)

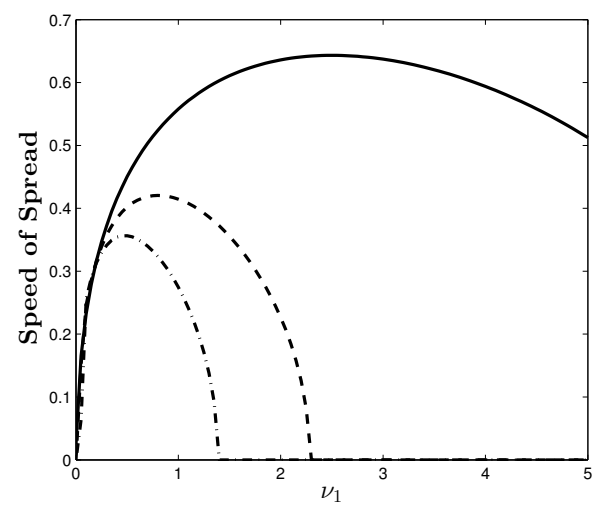

(b)

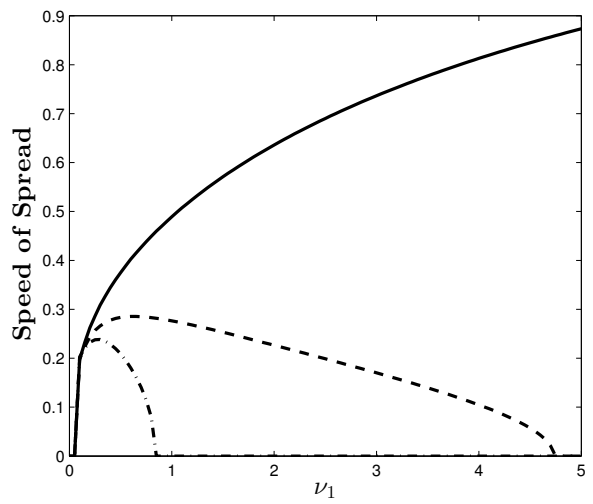

(c)

Figure 5.5: Spreading speed as a function of movement rate in good patches for $l_{2}=0.5$ (solid), $l_{2}=1$ (dashed), $l_{2}=1.5$ (dashed-dot) assuming interface conditions are given by (a) Case M, (b) Case S, (c) Case C. Fixed parameter values are: $\alpha_{1}=1, \alpha_{2}=1, \nu_{2}=2, \beta_{1}=1, \beta_{2}=1, l_{1}=2.5, r_{1}=2.5$, $r_{2}=0.6$, and $z=0$. 


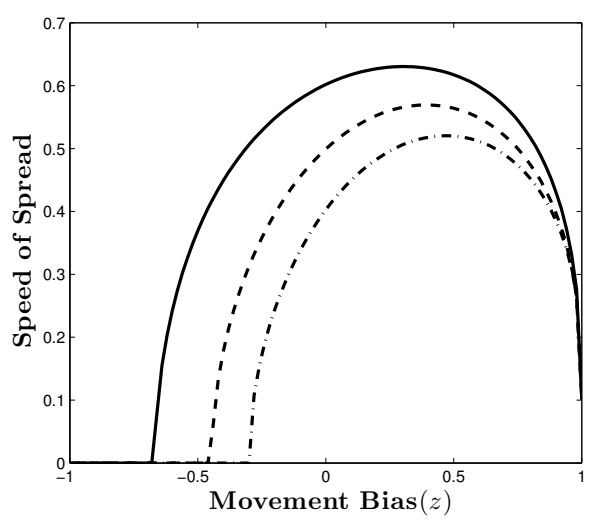

(a)

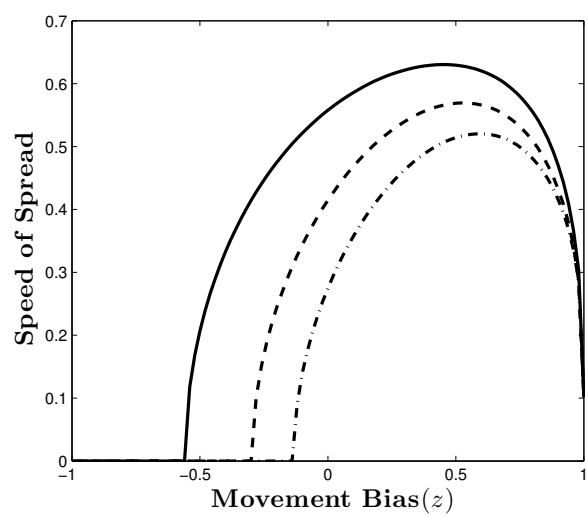

(b)

Figure 5.6: Spreading speed as a function of movement bias at an interface for $l_{2}=0.5$ (solid), $l_{2}=1$ (dashed), $l_{2}=1.5$ (dashed-dot) assuming interface conditions are given by (a) Case M, (b) Case S. Fixed parameter values are: $\alpha_{1}=1, \alpha_{2}=1, \nu_{1}=1, \nu_{2}=2, \beta_{1}=1, \beta_{2}=1, l_{1}=2.5, r_{1}=2.5$, and $r_{2}=0.6$.

However, if preference is too strong, individuals do not disperse from their current patch, and $c$ approaches zero. Increasing preference for bad patches results in the spreading speed approaching zero for all interface conditions, as persistence conditions are no longer met. The magnitude of the preference at which the speed goes to zero depends on movement behavior at an interface, with Case $\mathrm{M}$ producing a positive speed for stronger preference towards bad patches than Case S. 


\section{Chapter 6}

\section{The Interplay between Individual Dispersal Mechanisms and Barrier Zones: A Case Study on the Emerald Ash Borer}

\subsection{Introduction}

The spread of invasive species into new territory is a major concern for ecological diversity and population health, and it poses economic challenges and risk with millions of dollars required to contain or repair potential damage (With, 2002). Upon discovery of such invasions, the primary goal of forest managers and researchers is quarantine and eradication of the pest. However, eradication efforts can be extremely costly, and several of these efforts have failed (Liebhold and Tobin, 2008). Thus, managers have emphasized "slow-the-spread" campaigns where the primary goal is to reduce or delay adverse effects of the invasion. An example of such a strategy is the introduction of barrier zones. A barrier zone is a region, located ahead of the invasion front, in which 


\section{The Interplay between Individual Dispersal Mechanisms and Barrier Zones: A Case Study on the Emerald Ash Borer

the host plant of the invasive species is removed in the hope of slowing or preventing further spread.

Since the discovery of the EAB in 2002, efforts have been focused on eradicating the population with little success. More recently, management strategies have focused on slowing the spread of newly established populations, which requires a more thorough understanding of the local dispersal behavior of the EAB (Mercader et al., 2009). In this chapter, we develop a strategic model for the EAB and study how population spread rates depend on local dispersal behavior. We then examine the effectiveness of a barrier zone on slowing the spread of the EAB with a particular emphasis on the effect of movement behavior at the barrier zone interface.

Our strategic model for EAB is developed as follows. Although there is some evidence to suggest that EAB populations may take two generations to develop, we assume that the life-cycle consists of growth and dispersal phases synchronized in distinct, nonoverlapping generations. Thus we model population dynamics of the EAB using an IDE equipped with dispersal kernels derived in Chapters 2 and 3. In each generation, we count the number of eggs deposited; this quantity tells us which trees will be attacked in a given year and, secondly, it tells us the location of emerging adults at the beginning of the next generation. We use a difference equation to model the effect of EAB on the "health" of host trees. Mortality of host trees results from girdling of the host caused by larval consumption of phloem; we measure health of host trees as the amount of remaining phloem.

This chapter is organized as follows. In Section 6.2, we introduce our non-spatial and corresponding spatial strategic model for the spread of the EAB and interaction with host trees. Based on a thorough literature search, point-estimates along with a range for sensitivity analysis are provided for each parameter in our model in Section 6.2.1. In Section 6.3, we study the invasion of the EAB into an initial uniform density of phloem, and examine the dependence of spread rates on individual movement behavior. Spatial heterogeneity is introduced in Section 6.4 by considering a periodic 


\section{The Interplay between Individual Dispersal Mechanisms and Barrier Zones: A Case Study on the Emerald Ash Borer

landscape comprised of good and bad patches. In Section 6.4.1, we assume patch type does not affect dispersal. In Section 6.4.2, we introduce patch-dependent movement behavior and examine the effectiveness of barrier zones on slowing the spread of the EAB.

\subsection{A Strategic Model for the Emerald Ash Borer}

Let $t$ denote the year of the model, let $E_{t}$ denote the number of eggs deposited at the end of year $t$, and let $P_{t}$ denote the amount of remaining phloem at the end of year $t$. We consider the non-spatial model

$$
\begin{aligned}
& E_{t+1}=g\left(E_{t}, P_{t}\right)=\frac{r}{2}\left(1-\exp \left(-\zeta P_{t}\right)\right) m_{w} E_{t} \\
& P_{t+1}=h\left(E_{t}, P_{t}\right)=P_{t} \exp \left(-\eta m_{w} E_{t}\right),
\end{aligned}
$$

where $r$ is the average number of eggs laid per adult female, the factor $1 / 2$ accounts for a 50:50 sex ratio (Cappaert et al., 2005), $m_{w}$ is the over-wintering mortality of larvae, $\zeta$ is the parameter in the phloem-dependent failure rate for larval development, and $\eta$ is the consumption rate of phloem per larvae. The term $\left(1-\exp \left(-\zeta P_{t}\right)\right) m_{w} E_{t}$ gives the number of adults emerging in generation $t+1$, and $\exp \left(-\eta m_{w} E_{t}\right)$ measures the effect that larvae have on remaining phloem levels. Also note that an implicit assumption in (6.2.1) is that mating is not a limitation, which is a reasonable assumption given the aforementioned sex ratio.

Functions $g(E, P)$ and $h(E, P)$ in system (6.2.1) have the following qualitative properties. Function $g$ is increasing in $E$ and $P$, whereas $h$ is decreasing in $E$ and increasing in $P$. Thus, these functions represent the qualitative features that characterize predator-prey, consumer-resource and even infective-susceptible interactions.

The system in (6.2.1) is degenerate and has a continuum of equilibrium points 


\section{The Interplay between Individual Dispersal Mechanisms and Barrier Zones: A Case Study on the Emerald Ash Borer

given by $\left(0, P_{0}\right)$ with $P_{0} \geq 0$. The instability condition for $\left(0, P_{0}\right)$ is given by

$$
{ }_{2}^{r} m_{w}\left(1-\exp \left(-\zeta P_{0}\right)\right)>1,
$$

and local stability is unclear if (6.2.2) does not hold.

To introduce spatial considerations, let $\Omega$ denote the domain of interest and let

$k(x, y)$ be a dispersal kernel for the EAB; for example, as derived in Chapters 2 and 3. Since many univoltine species produce spatially distributed offspring by laying eggs, one may interpret the settling rate as an egg-deposition rate. Then the kernel gives the distribution of eggs deposited by a female with initial location $y$. Egg densities are modeled with an IDE given by

$$
E_{t+1}(x)=\int_{\Omega} k(x, y) g\left(E_{t}(y), P_{t}(y)\right) d y
$$

where $g(E, P)$ is as in system (6.2.1).

Many probability distributions have been fit to empirical data (Kot et al., 1996; Clark, 1998; Lockwood et al., 2002; Kot et al., 2004). In a spatially homogeneous landscape, the most common examples are the Gaussian and Laplace kernels. Because of the underlying mechanistic interpretation (see equation (2.2.8)), we choose the Laplace kernel and its variants for patchy landscapes as derived in Chapters 2 and 3.

Since trees do not move spatially on the timescale considered here, the equation for phloem levels holds at every $x$. Hence we study the system

$$
\begin{aligned}
& E_{t+1}(x)=\int_{\Omega} k(x, y) g\left(E_{t}(y), P_{t}(y)\right) d y=\frac{1}{2} \int_{\Omega} k(x, y) r\left(1-\exp \left(-\zeta P_{t}\right)\right) m_{w} E_{t} d y \\
& P_{t+1}(x)=h\left(E_{t}(x), P_{t}(y)\right)=P_{t}(x) \exp \left(-\eta m_{w} E_{t}(x)\right) .
\end{aligned}
$$

Our analysis of (6.2.4) proceeds as follows. We estimate parameters in the next section. In Section 6.3, we numerically analyze system (6.2.4) in a homogeneous 


\section{The Interplay between Individual Dispersal Mechanisms and Barrier Zones: A Case Study on the Emerald Ash Borer

landscape with initially uniform phloem density. We introduce spatial heterogeneity in Section 6.4 by considering a periodic landscape comprised of good and bad patches. In good patches, we assume there is a sufficient amount of host (phloem) for the population to grow, and in bad patches we assume there is no host (phloem) present. We begin our analysis with the Laplace kernel, assuming that patch type does not affect dispersal. We then include patch-dependent movement behavior by analyzing system (6.2.4) equipped with dispersal kernels derived in Chapters 2 and 3.

\subsubsection{Parameter Estimation}

Given its destructive nature, the EAB has been the focus of numerous research studies since its discovery in 2002. In recent years, many of these studies have been published (e.g. see Anulewicz et al., 2007; Mastro et al., 2007; McCullough and Siegert, 2007; Lyons et al., 2009; McCullough et al., 2009) and thus provide us with an avenue to estimate the parameters in system (6.2.1). For each parameter, we define a pointestimate (or default value) and a range for sensitivity analysis.

Through controlled lab experiments and field studies, egg deposition rates of EAB females appear to be well-understood, although there is some variability. The average number of eggs deposited by a single female is approximately 70 (Rutledge and Keena, 2012), with a minimum of approximately 30 (Anulewicz et al., 2008) and a maximum of 200 (Rutledge and Keena, 2012). To study the full scope of the invasion and to avoid introducing any bias in parameter estimates, we take the interval range of $r$ to be $(30,200)$ and the point estimate to be the observed mean value $r=70$.

Crosthwaite et al. (2011) recently published a detailed study of the overwintering physiology of the EAB. Their study focused on determining how sensitive larval survival is to cold temperatures and the supercooling point, the point at which larvae freeze to death. In optimal (non-cold) temperatures, survival ranged between 0 and $92 \%$. In general, over $50 \%$ of larvae successfully develop into adults, and lower sur- 


\section{The Interplay between Individual Dispersal Mechanisms and Barrier Zones: A Case Study on the Emerald Ash Borer

vival percentages were due to other factors such as woodpecker predation, pathogens, competition, and phloem levels, which are consistent with the findings of other studies (McCullough and Siegert, 2007). The effect of cold temperatures on larval survival depends on the severity of such temperatures. Between 2008 and 2010, the authors estimated the supercooling point to be between $-35.3^{\circ} \mathrm{Celsius}$ and $-11.3^{\circ} \mathrm{Celsius}$ depending on developmental stage. To ensure that some larvae survive, we take the interval range of $m_{w}$ to be $(0.1,1)$ with a point estimate given by the median value $m_{w}=0.55$.

McCullough and Siegert (2007) estimated that the total phloem level of an average-sized ash tree is approximately $22 \mathrm{~m}^{2}$, and the average number of adult EAB that could potentially emerge per $m^{2}$ of phloem is 89 . Since the average width of an ash tree is $10 \mathrm{~m}$, we estimate there is $2200 \mathrm{~m}^{2}$ of phloem per $\mathrm{km}$ of host. Using data from McCullough and Siegert (2007), Mercader et al. (2011a) estimated that an individual larvae would consume approximately $0.0113 \mathrm{~m}^{2}$ of phloem. To obtain a point estimate for $\eta$, we use two independent procedures.

First, we note that McCullough and Siegert (2007) obtained estimates of phloem levels available to EAB larvae from relatively healthy trees where egg densities are low. Therefore, for small $E$, the equation for $P$ in (6.2.1) may be approximated by

$$
P_{t+1} \approx P_{t}\left(1-\eta m_{w} E_{t}\right) \approx P_{t}-\eta P_{t} L_{t}
$$

where $L_{t}$ is the number of larvae. Since host trees are assumed to be relatively healthy, we take our point estimate for $P_{t}$ as $P_{0}$. Then, from equation (6.2.5), we see that the consumption rate of phloem per larvae is given by $C=\eta P_{0}$, and our point estimate for $\eta$ is $\eta=\frac{C}{P_{0}}=\frac{0.0113 \mathrm{~m}^{2} / \text { larvae }}{2200 \mathrm{~m}^{2} / \mathrm{km}} \approx 0.000005136 \frac{\mathrm{km}}{\text { larvae }}$.

Secondly, by re-arranging the equation for $P$ in system (6.2.5) as

$$
\frac{P_{t+1}}{P_{t}}=1-\eta m_{w} E_{t},
$$




\section{The Interplay between Individual Dispersal Mechanisms and Barrier Zones: A Case Study on the Emerald Ash Borer

we may estimate $\eta$ as the slope of the line connecting the points $(0,1)$ and $\left(m_{w} E^{*}, 0\right)$ where $\left(m_{w} E^{*}, 0\right)$ represents the total number of larvae that develop before a host dies. Since the average number of adults emerging per $m^{2}$ of phloem is 89 , we assume that 89 larvae are sufficient to consume $1 \mathrm{~m}^{2}$ of phloem and estimate $m_{w} E^{*}$ as

$$
m_{w} E^{*}=\frac{89 \text { larvae }}{m^{2} \text { phloem }} \frac{22 m^{2} \text { phloem }}{\text { host }} \frac{100 \text { hosts }}{k m}=195,800 \frac{\text { larvae }}{\mathrm{km}} .
$$

Therefore we obtain $\eta=\frac{1}{195,800} \approx 0.00000051073 \frac{\mathrm{km}}{\text { larvae }}$ which agrees surprisingly well with our estimate above. We take the interval range of $\eta$ to be roughly $20 \%$ on either side of the point estimate, i.e. $(0.000004,0.000006)$.

To the best of our knowledge, there are no data sets available from which $\zeta$ may be estimated directly. However, using available information on potential adult emergence from a given host allows us to obtain a reasonable estimate of $\zeta$. As outlined above, we estimate that approximately 195,800 adults could emerge per $\mathrm{km}$ of host. We obtain an estimate of $\zeta$ by simulating the non-spatial model in (6.2.1) over a wide range of possible $\zeta$-values to determine an interval range for $\zeta$ that results in the (cumulative) emergence of approximately 195,800 adults per $\mathrm{km}$ of host. In order to do this, we must provide an estimate of $E_{0}$.

Through anthropogenic dispersal, a number of EAB satellite colonies have been established at greater distances than possible through natural dispersal (Cappaert et al., 2005; Siegert et al., 2006). The establishment of these colonies at distances not obtainable through natural dispersal suggests that EAB populations are not subject to an Allee effect. Therefore we assume a single gravid female is sufficient for a population to establish on a single host and use the average number of eggs deposited by a gravid female as our estimate for the number of eggs necessary for a population to establish. Scaling up to 100 hosts per $\mathrm{km}$, we obtain $E_{0}=7000 \frac{\mathrm{eggs}}{\mathrm{km}}$.

Figure 6.1 shows a typical simulation of (6.2.1) where $\zeta=0.0002$ with other parameters given by the point estimates in Table 6.1. We observe that phloem levels 


\section{The Interplay between Individual Dispersal Mechanisms and Barrier Zones: A Case Study on the Emerald Ash Borer

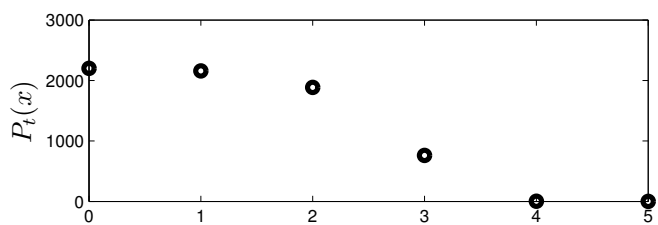

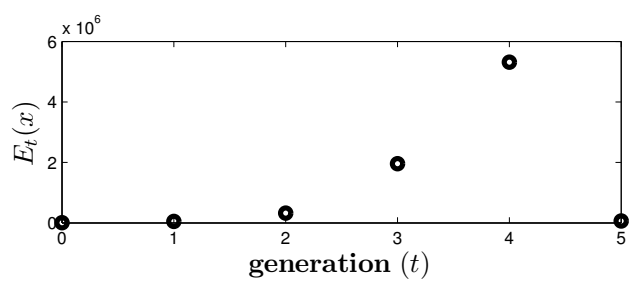

(a)

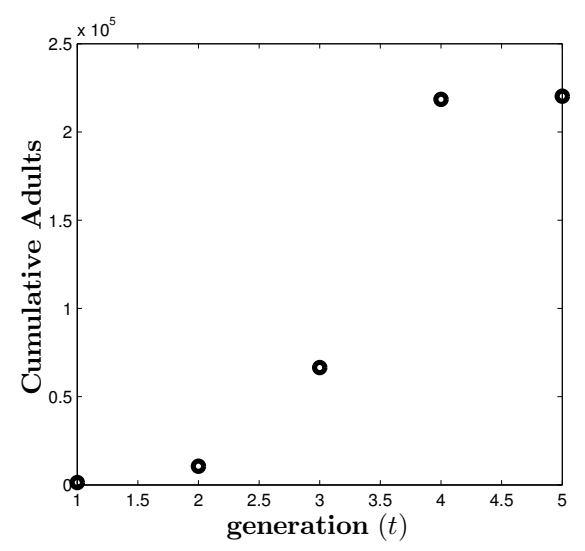

(b)

Figure 6.1: (a) Numerical solution of system (6.2.1) showing the number of eggs deposited and remaining phloem levels per generation (b) Cumulative adult emergence. Point estimates given in Table 6.1 were used.

decay to zero within approximately four years, which is consistent with the average time it takes for EAB to kill a host (Cappaert et al., 2005). Figure 6.1(b) shows that cumulative adult emergence is also consistent with the above estimate of approximately 195,800. Based on our numerical investigation, we take the interval range of $\zeta$ to be roughly $20 \%$ on either side of the point estimate; i.e., (0.00016, 0.00024). Note that a summary of point estimates and interval ranges for all parameters is given in Table 6.1.

\subsection{Homogeneous Landscape}

In this section, we study system (6.2.4) with $k$ given by Laplace kernel (1.3.3) with mean dispersal distance $\frac{1}{a}$, and on a homogeneous one-dimensional landscape. Field studies have found that approximately $90 \%$ of EAB females deposit their eggs within $500 m$ of their emergence site (Mercader et al., 2009; Siegert et al., 2010). The mean dispersal distance is then estimated using the dispersal success function (see equation 


\section{The Interplay between Individual Dispersal Mechanisms and Barrier Zones: A Case Study on the Emerald Ash Borer

Table 6.1: Parameter estimates for non-spatial EAB model

\begin{tabular}{|c|c|c|}
\hline Parameter (units) & Point Estimate (range) & Reference \\
\hline$r\left(\frac{\# \text { eggs deposited }}{\# \text { adults }}\right)$ & $70(30,200)$ & $\begin{array}{l}\text { Anulewicz et al. (2008) } \\
\text { Rutledge and Keena (2012) }\end{array}$ \\
\hline$m_{w}\left(\frac{\# \text { adults }}{\# \text { eggs deposited }}\right)$ & $0.55(0.1,1)$ & Crosthwaite et al. (2011) \\
\hline$\eta\left(\frac{\mathrm{km} \text { host }}{\# \text { larvae }}\right)$ & $0.0000051(0.000004,0.000006)$ & $\begin{array}{l}\text { McCullough and Siegert (2007) } \\
\text { Mercader et al. (2011b) }\end{array}$ \\
\hline$\zeta\left(\frac{\mathrm{km} \text { host }}{\mathrm{m}^{2} \text { phloem }}\right)$ & $0.0002(0.00016,0.00024)$ & - \\
\hline$P_{0}\left(\frac{m^{2} \text { phloem }}{k m \text { host }}\right)$ & 2200 & McCullough and Siegert (2007) \\
\hline$E_{0}\left(\frac{\# \text { eggs }}{k m \text { host }}\right)$ & 7000 & - \\
\hline
\end{tabular}

(3.4.1)), and we obtain $\frac{1}{a}=0.22 \mathrm{~km}$.

Linearizing system (6.2.4) about $\left(0, P_{0}\right)$, we find that the equation for $E$ decouples; namely,

$$
E_{t+1}(x) \approx \int_{\Omega} k(x, y) \frac{r}{2}\left(1-e^{-\zeta P_{0}(y)}\right) m_{w} E_{t}(y) d y .
$$

According to the theory for spreading speeds (see Section 1.3.2), we find

$$
c^{*}=\min _{s>0}\left\{\frac{1}{s} \ln \left[0.5 r m_{w}\left(1-e^{-\zeta P_{0}}\right) M(s)\right]\right\},
$$

where $M(s)=\frac{1}{1-\frac{s^{2}}{a^{2}}}$ is the moment-generating function for the Laplace kernel. Equation (6.3.2) cannot be minimized analytically, so one must resort to numerical techniques.

It is not clear whether linear determinacy holds for the system in (6.2.4), but in all our numerical simulations, we found that the spreading speed of system (6.2.4) agreed with the linearized speed $c^{*}$ in (6.3.2). An analytic proof of this conjecture is left as future work.

Since the system in (6.2.4) is coupled and nonlinear, we investigate the qualitative 


\section{The Interplay between Individual Dispersal Mechanisms and Barrier Zones: A Case Study on the Emerald Ash Borer

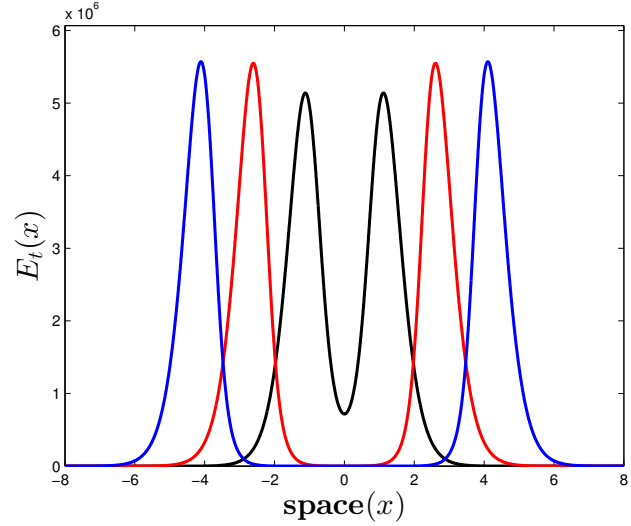

(a) Eggs

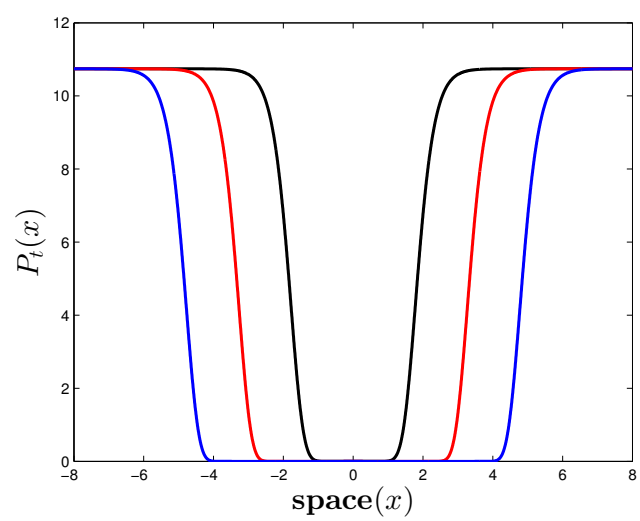

(b) Phloem

Figure 6.2: Numerical solution of system (6.2.4) showing a traveling pulse solution for the spread of EAB into a homogeneous landscape of host trees with uniform initial density after $t=6$ (black), $t=8$ (red), and $t=10$ (blue) generations. Parameter values are given by the point estimates listed in Table 6.1. The spatial unit is $\mathrm{km}$ and the mean dispersal distance is $\frac{1}{a}=0.22 \mathrm{~km}$.

properties of this system numerically. To ensure consistency among the simulations of model (6.2.4), point estimates provided in Table 6.1 for $E_{0}$ and $P_{0}$ will be used for all simulations. Furthermore, we assume $E_{0}$ is uniformally distributed over one $\mathrm{km}$ of host and an initial uniform phloem density that results in $2200 \mathrm{~m}^{2}$ of phloem per $\mathrm{km}$ of host.

For our initial investigation, we simulate (6.2.4) for the point estimates given in Table 6.1. Since egg densities depend on phloem levels from the previous generation, we plot egg densities after $t=6,8$ and 10 generations with corresponding phloem levels from the previous generation. Observe from Figure 6.2 that as EAB invades the host population, a traveling pulse solution forms where the population maintains its shape while traveling at a constant speed. The traveling pulse solution was obtained over a wide range of parameter space and thus appears to be a robust solution of this system. 


\section{The Interplay between Individual Dispersal Mechanisms and Barrier Zones: A Case Study on the Emerald Ash Borer

To further study the invasion process, we are interested in the following quantitative properties of the pulse and their dependence on model parameters: the speed and width of the pulse. The width of the pulse allows us to quantify the size/severity of the invasion and the speed tells us how fast the population is spreading as well as the location of new populations.

Due to uncertainty in model parameters, we wish to explore a reasonably large portion parameter space of model (6.2.4). To do this, we employ a sampling technique known as Latin hypercube sampling (LHS) (Iman and Conover, 1980). To implement the LHS procedure, each parameter is assigned a probability density function. Since complete data sets are unavailable, we choose a uniform distribution for all parameters, which is a natural first choice (Marino et al., 2008). Each probability density function is then partitioned into $\mathcal{N}$ non-overlapping, equiprobable intervals that are sampled without replacement. Assuming there are $\mathcal{K}$ parameters, the LHS procedure results in an $\mathcal{N} \times \mathcal{K}$ matrix representation of parameter space.

In order to assess the variability in model outputs and identify key model parameters, we employ a sensitivity analysis technique known as partial rank correlation coefficients (PRCCs) (Iman and Conover, 1980; Marino et al., 2008). Partial correlation measures the strength of the linear relationship between an input variable, say $x_{i}$, and an output variable, say $y$, after the linear effects of remaining input variables on $y$ are discounted. To reduce the effect of nonlinear data, the data are ranked where the smallest value is given a rank of 1 , next smallest value a rank of 2 , and so on with repeated values assigned an average rank.

To determine the PRCC between $x_{i}$ and $y$, two linear regression models are calculated. The first model is a linear regression of $x_{i}$ with the remaining inputs and the second is the linear regression of $y$ with the remaining inputs. The PRCC is then the partial correlation coefficient between the residuals $r_{x_{i}}=\left(x_{i}-\hat{x}_{i}\right)$ and $r_{y}=(y-\hat{y})$, where $\hat{x}_{i}$ and $\hat{y}$ are the linear regression models previously described. 


\section{The Interplay between Individual Dispersal Mechanisms and Barrier Zones: A Case Study on the Emerald Ash Borer

Specifically, the PRCC is given by

$$
\rho_{x_{i}, y}=\frac{\operatorname{Cov}\left(r_{x_{i}}, r_{y}\right)}{\sqrt{\operatorname{Var}\left(r_{x_{i}}\right) \operatorname{Var}\left(r_{y}\right)}} .
$$

PRCC scatter plots are shown in Figure 6.3 for parameters $r, m_{w}, \eta, \zeta$, and the spreading speed of the pulse for $\mathcal{N}=1000$ simulations. The abscissa represents the residuals of the linear regression between the rank-transformed values of the indicated parameter versus the rank-transformed values of the other input parameters. The ordinate represents the residuals of the linear regression between the rank-transformed spreading speed values and the remaining input parameters. The title of each plot gives the PRCC with corresponding p-value. A strong, positive correlation exists between the pulse speed and $r, m_{w}$ and $\zeta$, while there is no correlation between the speed and $\eta$.

The strong correlations between speed and $r, m_{w}$ and $\zeta$ are consistent with biological intuition. Indeed, near the front of a spreading population, egg densities are low and phloem levels are relatively abundant. Thus it seems reasonable that the pulse speed has strong correlations with parameters that affect growth $\left(r, m_{w}\right)$ and survival $(\zeta)$ and little correlation with consumption of phloem by larvae $(\eta)$ since egg densities are low. The correlations are also consistent with mathematical theory since we know the linearized spreading speed for an EAB invasion depends on $r, m_{w}$ and $\zeta$, but not $\eta$ (see equation (6.3.2)).

The term $r m_{w}\left(1-\exp \left(-\zeta P_{0}\right)\right)$ is the maximum rate of increase of a population per number of eggs deposited (or the maximum intrinsic growth rate). In Figure 6.4(a), we plot the numerical and analytic (see equation (6.3.2)) spreading speeds on the ordinate and $r m_{w}\left(1-\exp \left(-\zeta P_{0}\right)\right)$ on the abscissa for the LHS used to generate Figure 6.3. Observe that both spreading speeds appear to increase in a square root like fashion as the intrinsic growth rate increases, which agrees with other models of population spread (Skellam, 1951; Kot et al., 1996). As well, note that the numerical 


\section{The Interplay between Individual Dispersal Mechanisms and Barrier Zones:
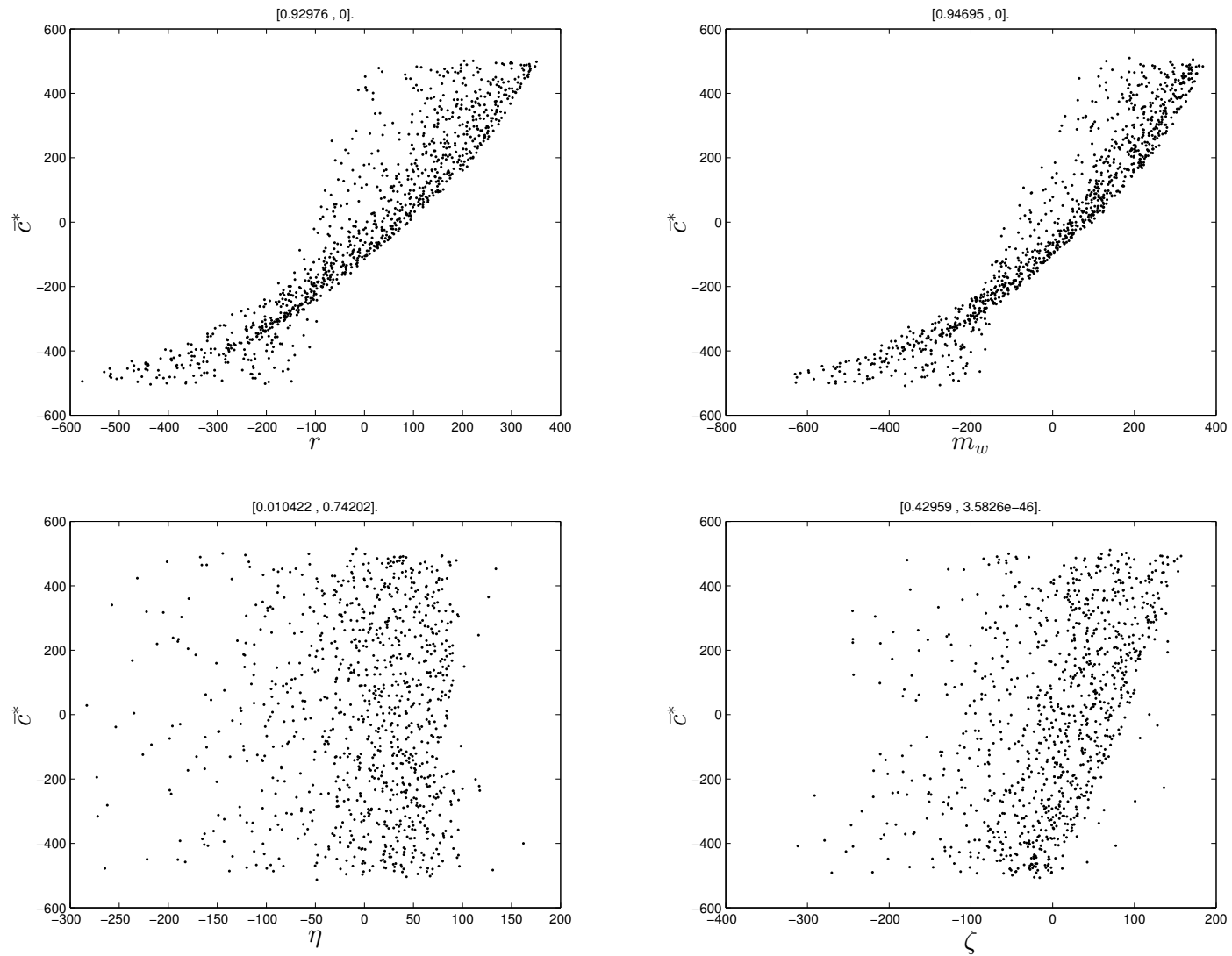

Figure 6.3: PRCC plots of model parameters $r, m_{w}, \eta$, and $\zeta$. The abscissa represents the residuals of the linear regression between the rank-transformed values of the indicated parameter versus the rank-transformed values of the other input parameters. The ordinate represents the residuals of the linear regression between the rank-transformed spreading speed values and the remaining input parameters. The title of each plot gives the PRCC with corresponding p-value. The mean dispersal distance is $\frac{1}{a}=0.22 \mathrm{~km}$. 


\section{The Interplay between Individual Dispersal Mechanisms and Barrier Zones: A Case Study on the Emerald Ash Borer

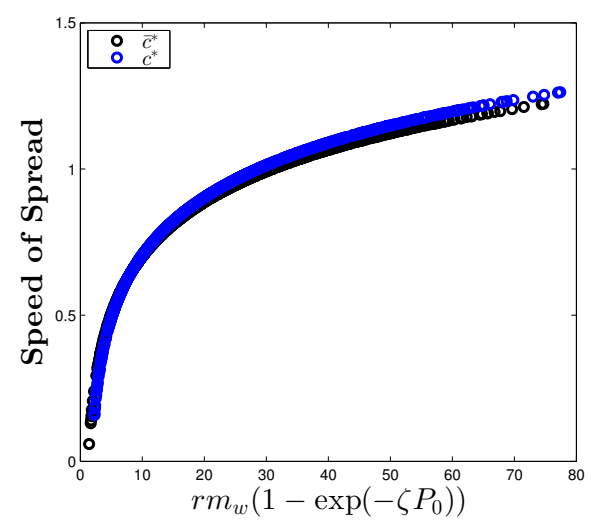

(a)

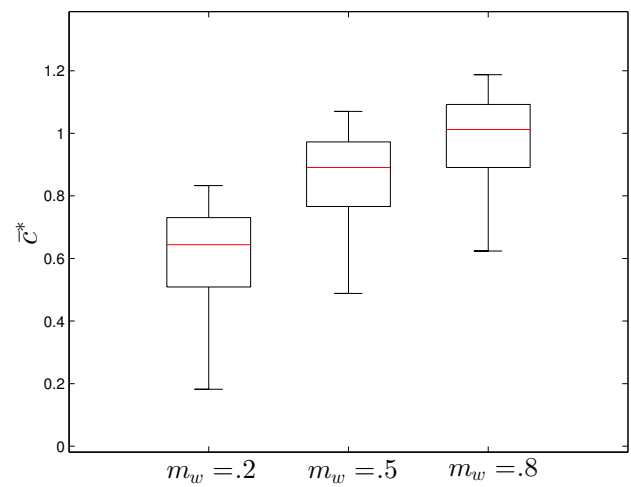

(b)

Figure 6.4: Left Panel: Numerical (black dots) and analytic (blue dots) spreading speed versus the maximum intrinsic growth rate. We observe that both speeds appear to increase in a square root like fashion, and the numerical speed agrees well with the speed obtained from the linearization. Parameter values were given by the LHS used to generate Figure 6.3. Right Panel: Boxplots of numerically obtained spreading speeds for different values of $m_{w}$ with other parameters given by the LHS used to generate Figure 6.3. The mean dispersal distance is $\frac{1}{a}=0.22 \mathrm{~km}$.

speed agrees well with the linearized expression $c^{*}$ in (6.3.2).

Of the three parameters that comprise the intrinsic growth rate, the over-wintering survival of larvae is the one that is most likely to be affected by management strategies as well as other factors such as woodpecker predation and competition. The sensitivity of the spreading speed to $m_{w}$ is shown in Figure 6.4(b). The spreading speed has a low sensitivity to the overwintering survival rate. For example, a four-times decrease in $m_{w}$ resulted in only a one and a half times decrease in the spreading speed.

In Figure 6.5, PRCC scatter plots are shown for parameters $r, m_{w}, \eta, \zeta$, and the width of the pulse. To measure the width, denote $e^{*}$ as the threshold level below which the invading population is not detectable, and let $x_{1}$ and $x_{2}$ denote the spatial locations at which $E_{t}(x) \equiv e^{*}$ along the pulse. For each value of $t$, the pulse width is then given by $\left|x_{1}-x_{2}\right|$. For each parameter set, the average value of the pulse width 


\section{The Interplay between Individual Dispersal Mechanisms and Barrier Zones: A Case Study on the Emerald Ash Borer

is used. We observe strong, positive correlations between the pulse width and $r, m_{w}$, and $\zeta$ and a weak, negative correlation with $\eta$. The strong correlations seem entirely reasonable since, at the front of the pulse, egg densities are relatively low and phloem levels abundant; conversely, behind the pulse, phloem levels are low; see Figure 6.2. This result suggests the width of the pulse is strongly correlated with the speed of the pulse, which one would expect. The correlation between the width and $\eta$ agrees with biological intuition since $\eta$, the consumption rate of phloem per larvae, affects where adults deposit their eggs in the next generation, but not the current one.

The dependence of the pulse width on the maximum intrinsic growth rate can be observed from Figure 6.6(a). It appears that the pulse width increases in a square root like fashion with the maximum intrinsic growth rate. The width of the pulse has a low sensitivity to the overwintering survival of larvae as can be observed in Figure 6.6(b). A four-times decrease in the overwintering survival of larvae results in the mean of the pulse width decreasing from approximately $9.7 \mathrm{~km}$ to $8.5 \mathrm{~km}$.

\subsection{A Periodic Landscape}

\subsubsection{Homogeneous Dispersal}

In this section, we study system (6.2.4) on an idealized, infinite, one-dimensional landscape that is periodically varying with good patches of length $l_{1}$ and bad patches of length $l_{2}$ so that the period of the landscape is $l=l_{1}+l_{2}$. In bad patches, there are no host trees present and conditions do not support local population growth. In good patches, phloem and egg densities are modeled as in (6.2.1). In this section, we assume that individuals show no patch-dependent dispersal behavior. The system we study is (6.2.4) where $k$ is given by a Laplace kernel (1.3.3) with mean dispersal 


\section{The Interplay between Individual Dispersal Mechanisms and Barrier Zones:
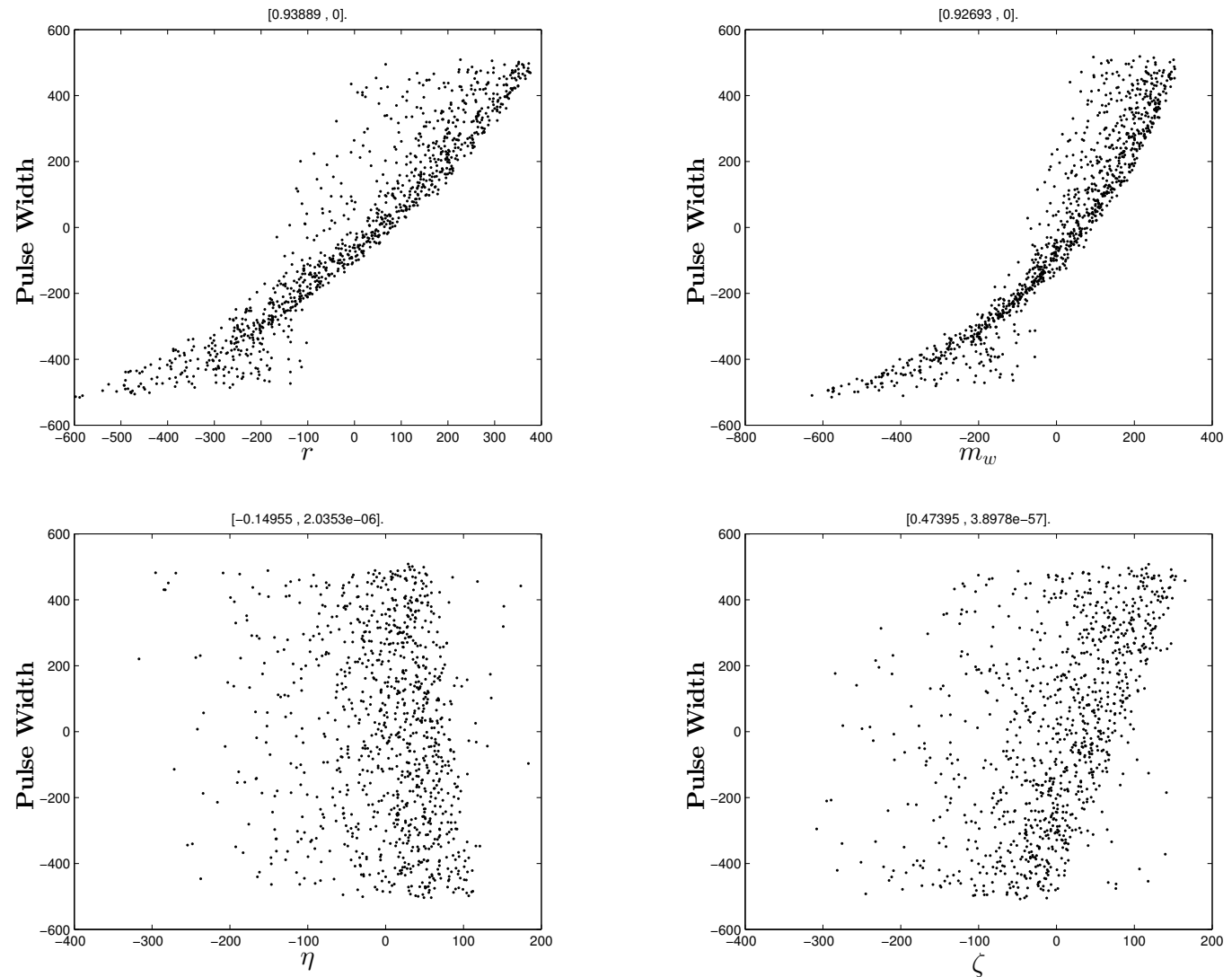

Figure 6.5: PRCC plots of model parameters $r, m_{w}, \eta$, and $\zeta$. The abscissa represents the residuals of the linear regression between the rank-transformed values of the indicated parameter versus the rank-transformed values of the other input parameters. The ordinate represents the residuals of the linear regression between the rank-transformed pulse width values and the remaining input parameters. The title of each plot gives the PRCC with corresponding p-value. The mean dispersal distance is $\frac{1}{a}=0.22 \mathrm{~km}$. 


\section{The Interplay between Individual Dispersal Mechanisms and Barrier Zones: A Case Study on the Emerald Ash Borer
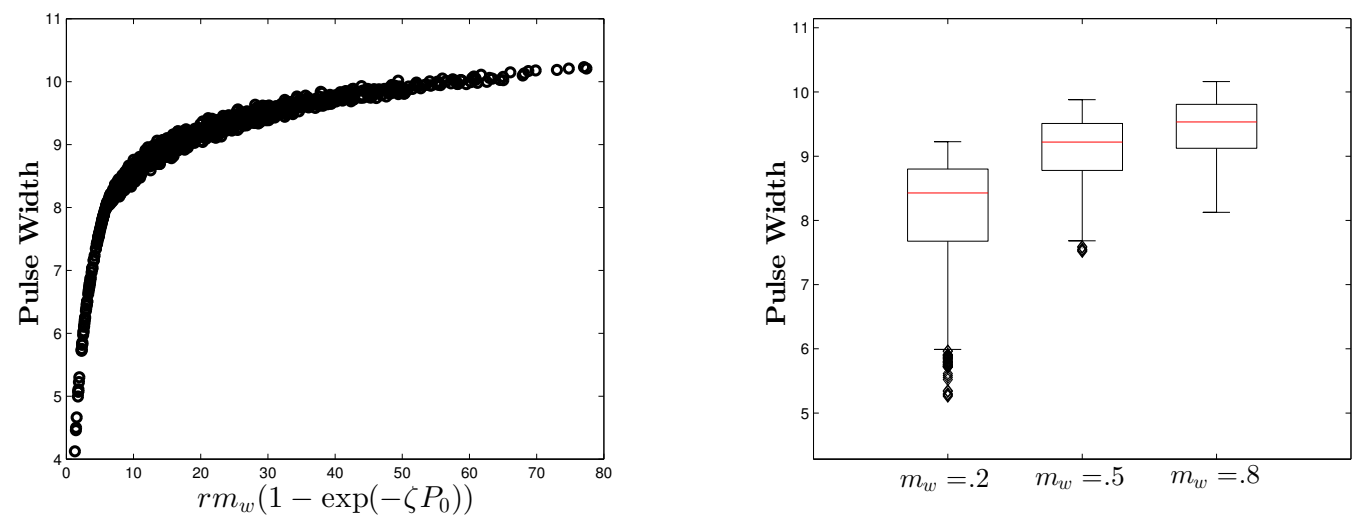

Figure 6.6: Left Panel: Pulse width versus the maximum intrinsic growth rate. We observe that the width appears to increase in a square root like fashion. Parameter values were given by the LHS used to generate Figure 6.3. Right Panel: Boxplots of pulse width for different values of $m_{w}$ with other parameters given by the LHS used to generate Figure 6.3. The mean dispersal distance is $\frac{1}{a}=0.22 \mathrm{~km}$.

distance $\frac{1}{a}=0.22 \mathrm{~km}$. The initial phloem density $P_{0}$ is given by

$$
P_{0}(x)=\left\{\begin{array}{c}
P_{0}, \quad x \in\left(-\frac{l_{1}}{2}, \frac{l_{1}}{2}\right)+l \mathbb{Z} \\
0, x \in\left(\frac{l_{1}}{2}, l-\frac{l_{1}}{2}\right)+l \mathbb{Z},
\end{array}\right.
$$

and $E_{0}$ is as in system (6.2.4).

As noted by several authors (VanKirk, 1995; Fahrig, 2002; With, 2002; Dewhirst and Lutscher, 2009), the scale of landscape heterogeneity can have significant effects on population spread rates. Following the procedure outlined in Section 4.5, we scale the spatial variable by the period of the landscape and introduce the dimensionless parameter $\hat{p}=\frac{l_{1}}{l}$, the percentage of good habitat. Low values of $\hat{p}$ correspond to high fragmentation levels and large loss of suitable habitat whereas large values correspond to low fragmentation levels and low loss of suitable habitat.

Dewhirst and Lutscher (2009) used homogenization techniques to derive an ap- 


\section{The Interplay between Individual Dispersal Mechanisms and Barrier Zones: A Case Study on the Emerald Ash Borer

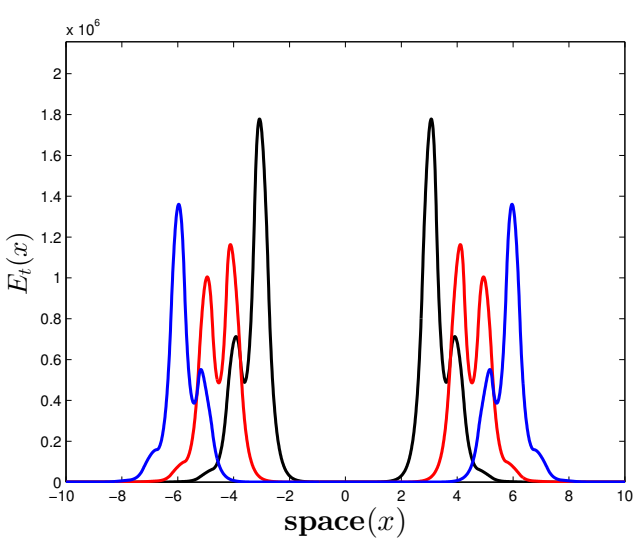

(a) Eggs

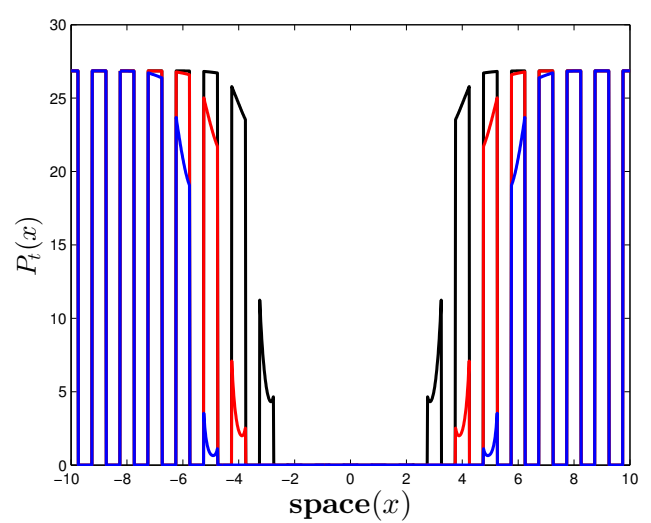

(b) Phloem

Figure 6.7: Numerical solution of system (6.4.1) showing (a) spread of EAB into a periodic landscape of host trees with uniform initial density in good patches after $t=10$ generations (black), $t=12$ generations (red) and $t=14$ generations (blue) and (b) remaining phloem densities after $t=9$ generations (black), $t=11$ generations (red) and $t=13$ generations (blue). Parameter values are given by the point estimates listed in Table 6.1 with $l_{1}=l_{2}=1$ and mean dispersal distance $\frac{1}{a}=0.22 \mathrm{~km}$.

proximate formula for spreading speeds in patchy landscapes. Following these authors and assuming linear determinacy holds, we find that the approximate spread rate is given by

$$
c^{*}=\min _{s>0}\left\{\frac{1}{s} \ln \left[0.5 \hat{p} r m_{w}\left(1-e^{-\zeta P_{0}}\right)\right]\right\} .
$$

As above for system (6.2.4), we study the properties of this system numerically. The plot in Figure 6.7(a) shows the spatial profile of the EAB invasion into a periodic landscape of host trees, and the plot in Figure 6.7(b) shows remaining phloem levels. Since egg densities depend on phloem levels in the previous generation (see system (6.2.4)), we plot egg densities after $t=10,12$ and 14 generations and remaining phloem levels for each prior generation. Observe that the peaks of the pulse are not constant due to the non-uniform phloem density.

To further illustrate the properties of system (6.2.4) in a periodic landscape, we 


\section{The Interplay between Individual Dispersal Mechanisms and Barrier Zones: A Case Study on the Emerald Ash Borer

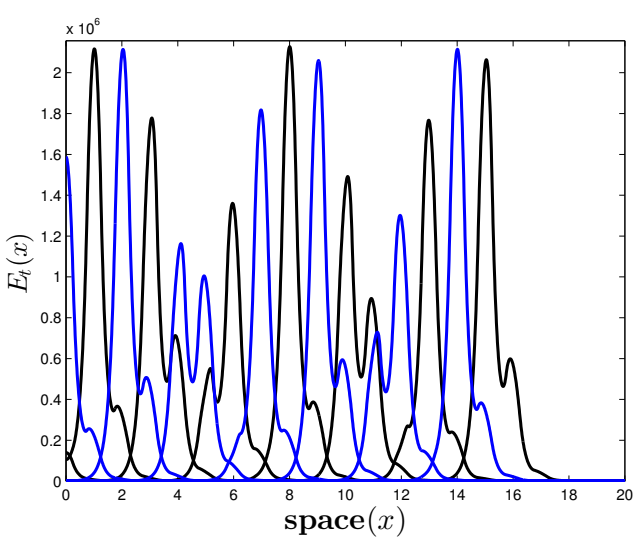

(a)

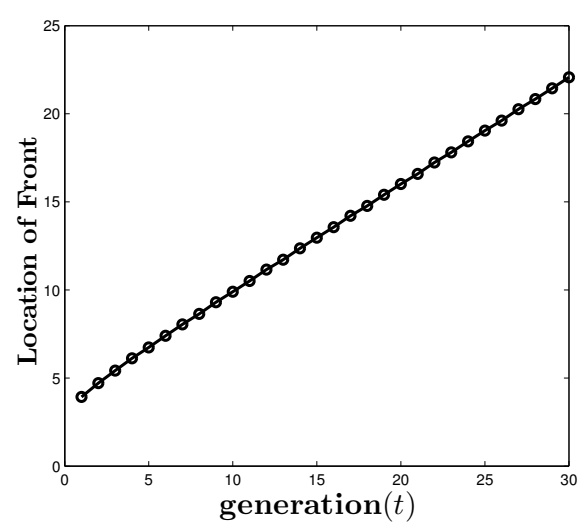

(b)

Figure 6.8: (a) Numerical simulation of system (6.2.4) for 30 generations, showing the spatial profile of the EAB invasion every second generation alternating between black and blue curves. The plot shows that the spatial profile of the invasion resembles that of a pulse, however, the shape of each pulse is not constant due to non-uniform phloem densities. (b) Front location of the EAB invasion showing that the speed of the pulse is constant. Parameter values are given by the point estimates listed in Table 6.1 with $l_{1}=l_{2}=1$ and mean dispersal distance $\frac{1}{a}=0.22 \mathrm{~km}$.

simulated the model for 30 generations and we plot the spatial profile of the EAB invasion every second generation. Observe from Figure 6.8(a) that the spatial profile of the invasion resembles that of a pulse; however, the shape of each pulse is not constant due to non-uniform phloem densities. The plot in Figure 6.8(b) shows that the invasion moves with constant speed.

Figure 6.9 shows PRCC plots of the parameters $r, m_{w}, \zeta$, and $\hat{p}$ versus the speed of the pulse. Since equation (6.4.2) does not depend on $\eta$, we exclude this parameter from our analysis. As expected, the strongest correlation exists between the percentage of good habitat, $\hat{p}$, and the speed. Biologically, large population spread rates are positively correlated with high percentages of good habitat. The correlation between the speed and other parameters is not as strong as in Figure 6.3 due to the introduction of bad patches. However, the speed is still strongly correlated with 


\section{The Interplay between Individual Dispersal Mechanisms and Barrier Zones: A Case Study on the Emerald Ash Borer

average deposition rates $(r)$ and overwinter survival of larvae $\left(m_{w}\right)$. The spreading speed increases in a square root like fashion as the product of the maximum intrinsic growth rate and $\hat{p}$ increases, as shown in Figure 6.10(a).

As discussed in Section 6.1, we are interested in studying the effect of barrier zones on slowing the spread of EAB. In our framework, we interpret bad patches as barrier zones, thus having an idealized situation where the front of the spreading population periodically encounters a barrier zone. To measure the efficiency of a barrier zone, we follow Sharov and Liebhold (1998) and examine the relative reduction in the spreading speed for barrier zones of different size. The relative reduction in the spreading speed is given by

$$
\bar{c}_{r}^{*}=1-\frac{\bar{c}^{*}}{\bar{c}_{\max }},
$$

where $\bar{c}_{\max }$ is the maximum spreading speed; i.e., the spreading speed in a homogeneous landscape.

Figure 6.10(b) shows the relative reduction in spreading speeds for three different levels of landscape fragmentation: $\hat{p}=0.5, \hat{p}=0.25$, and $\hat{p}=0.1$. Reducing the percentage of good habitat by $80 \%$ results in an average reduction of the spreading speed by approximately $65 \%$. The efficiency of barrier zones increases as the overwintering survival of larvae decreases; see Figure 6.10(c). Thus the greater the reduction in the number of larvae successfully developing into an adult is, the greater the reduction in spreading speeds caused by a barrier zone. Also, note that, as $m_{w} \rightarrow 1$, the relative reduction in spreading speeds decreases significantly, unless the relative width of a barrier zone is large.

For sake of brevity, we do not show PRCC plots of the model parameters versus the width of the pulse nor plots showing the relative reduction in the width of the pulse. The results are similar to the spreading speed in that the strongest correlation is between the width and $\hat{p}$, and the correlations with other parameters are not as 


\section{The Interplay between Individual Dispersal Mechanisms and Barrier Zones:
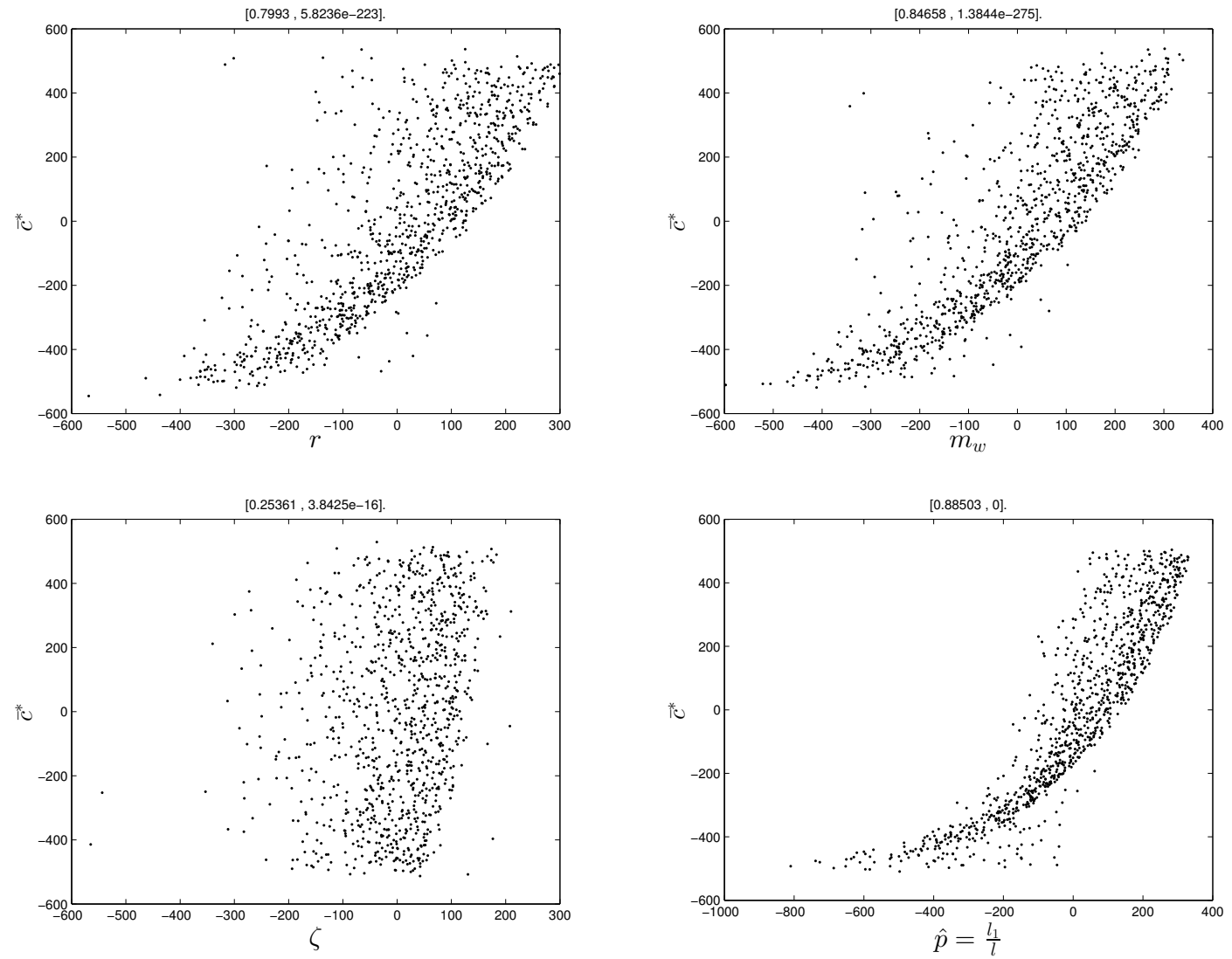

Figure 6.9: PRCC plots of model parameters $r, m_{w}, \zeta$, and $\hat{p}$. The abscissa represents the residuals of the linear regression between the rank-transformed values of the indicated parameter versus the rank-transformed values of the other input parameters. The ordinate represents the residuals of the linear regression between the rank-transformed approximate spreading speeds and the remaining input parameters. The title of each plot gives the PRCC with corresponding p-value. The mean dispersal distance is $\frac{1}{a}=.22 \mathrm{~km}$. 


\section{The Interplay between Individual Dispersal Mechanisms and Barrier Zones: A Case Study on the Emerald Ash Borer

strong, due to the introduction of bad patches. Also, the relationship between the relative reduction in the pulse width and the size of the barrier zone produces similar figures obtained for the speed shown in Figures 6.10(b) and 6.10(c).

\subsubsection{Heterogenous Dispersal}

In this section, we assume individuals exhibit patch-dependent movement behavior and equip system (6.4.1) with kernels derived in Chapters 2 and 3. Dispersal kernels obtained from our procedure depend on which set of interface conditions we choose (see equations (2.4.8)-(2.4.10)). For illustrative purposes, we choose Cases M (2.4.8) and C (2.4.10), and examine how previous results obtained in Sections 6.3 and 6.4.1 depend on dispersal parameters (see model (2.1.2)) and behaviour at an interface.

Since every different parameter combination in model (2.1.2) generates a unique dispersal kernel and each simulation is computationally intensive, we do not consider PRCCs in this section. Rather, we focus on how the qualitative behavior of system (6.4.1) depends on individual behavior at an interface and examine the interplay between barrier zones and individual dispersal mechanisms.

The consideration of patch-dependent dispersal behavior introduces an additional seven parameters. To simplify our analysis, we introduce the effective motility coefficient, $\hat{\nu}=\frac{\nu_{2}}{\nu_{1}}$, and the effective settling rate, $\hat{\alpha}=\frac{\alpha_{2}}{\alpha_{1}}$. Then, $\hat{\nu}>1$ corresponds to individuals dispersing slowly through good patches whereas $\hat{\nu}<1$ corresponds to fast movement in good patches; an analogous interpretation of $\hat{\alpha}$ can be made. Movement bias at an interface is modeled by a parameter, $z$; see Chapter 2. Furthermore, since we include larval mortality and it is likely that gravid females deposit the majority of their eggs before death, we assume that $\beta_{i}=0, i=1,2$.

Figure 6.11 shows a numerical simulation of system (6.4.1) for Cases C (lefthand column) and $\mathrm{M}$ (right-hand column), assuming $\hat{\alpha}=1, \hat{\nu}>1$, and $z=0$ with other parameters given by the point estimates listed in Table 6.1. We observe 


\section{The Interplay between Individual Dispersal Mechanisms and Barrier Zones:

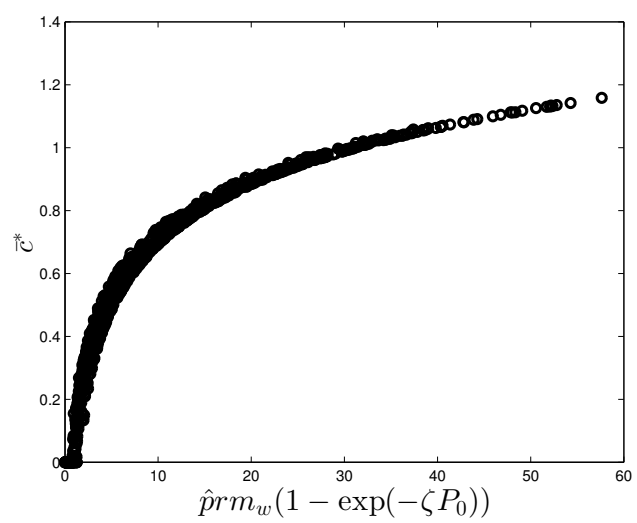

(a)

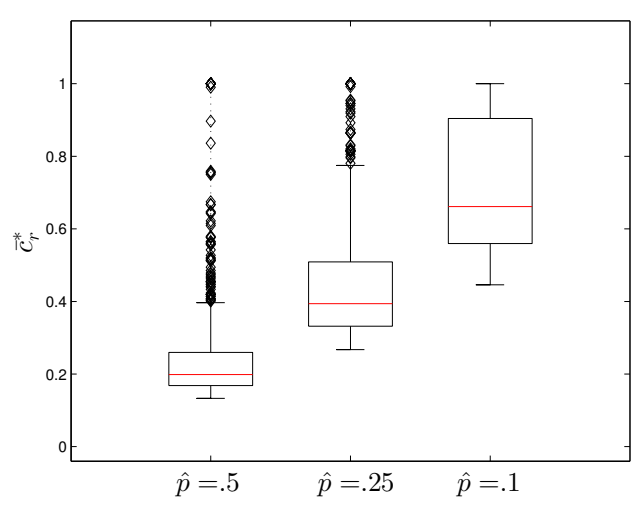

(b)

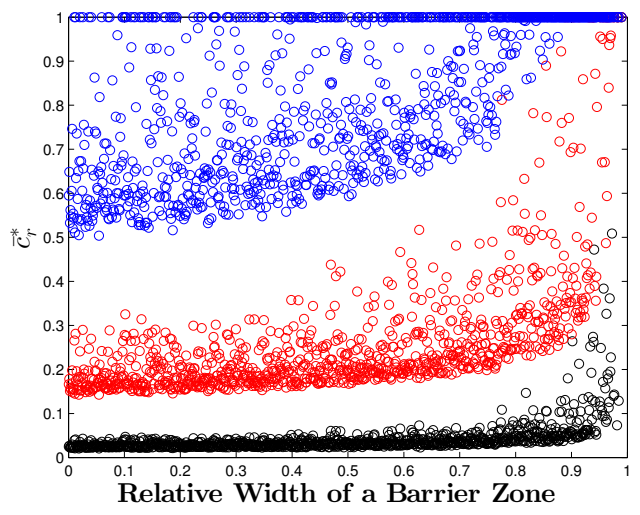

(c)

Figure 6.10: Panel (a): Numerical spreading speed increases in a square root like fashion as the product of the maximum intrinsic growth rate, and $p$ increases in a patchy landscape with $l_{1}=l_{2}=1$. Panel (b): Relative Reduction in spreading speed, $1-\frac{\bar{c}^{*}}{\bar{c}_{h}^{*}}$, for (a) $\hat{p}=0.5$ (b) $\hat{p}=0.25$ (c) $\hat{p}=0.1$. Panel (c): Relative reduction in spreading speed decreases with relative width of a barrier zone as overwintering survival of larvae increases. The blue, red, and black dots correspond to $m_{w}=0.1, m_{w}=0.5$, and $m_{w}=0.9$, respectively. Other parameter values are given by the LHS used to generate Figure 6.9. The mean dispersal distance is $\frac{1}{a}=0.22 \mathrm{~km}$. 


\section{The Interplay between Individual Dispersal Mechanisms and Barrier Zones: A Case Study on the Emerald Ash Borer

that the spatial profile of the EAB invasion is sensitive to the interface conditions. In particular, we see that significantly more eggs are deposited when individuals disperse according to Case M. Since $\hat{\nu}>1$, female beetles dispersing according to Case $\mathrm{C}$ have a higher probability of settling in bad patches, resulting in lower residency times in good patches and fewer eggs deposited. To further illustrate the qualitative behavior of system (6.4.1), we simulated the model over 30 generations for Case M. As before, the shape of the invasion is not preserved; however, the invasion does advance at a constant speed; see Figure 6.12.

To illustrate the effect of movement bias on the pulse, we simulate system (6.4.1) assuming a bias towards good patches given by $z=0.5$ with other parameters as listed in the caption of Figure 6.13. The bias towards good patches results in a lower spread rate through good patches, but larger egg densities as females spend more time in good patches thus depositing more eggs.

We now study the interplay between individual dispersal mechanisms and barrier zones. For sake of brevity, we simplify our analysis as follows. Two values of landscape fragmentation are used: $\hat{p}=0.5$ (moderate fragmentation) and $\hat{p}=0.1$ (high fragmentation). Moderate $\hat{p}$ values correspond to barrier zones being (approximately) the same size as that of good patches whereas large $\hat{p}$ values correspond to barrier zones being much larger than good patches. For each $\hat{p}$ value, we examine the relative reduction in spread rates under two dispersal scenarios. First, we assume there is no movement bias $(z=0)$ at an interface and calculate $\bar{c}_{r}^{*}$ assuming $\hat{\nu}>1$ or $\hat{\nu}<1$. Secondly, we assume individuals move slower in good patches so that $\hat{\nu}>1$ and examine the interplay between barrier zones and a movement bias at the interface. Specifically, we calculate $\bar{c}_{r}^{*}$ assuming $z=0.5$ (strong bias towards good patches) and $z=-0.5$ (strong bias towards bad patches). For all simulations, it is assumed that $\hat{\alpha}<1$. Since the likelihood of EAB depositing their eggs on non-ash trees is lower than on ash trees (Anulewicz et al., 2006, 2007), this assumption is reasonable. Below, we illustrate the interplay between barrier zones and individual 

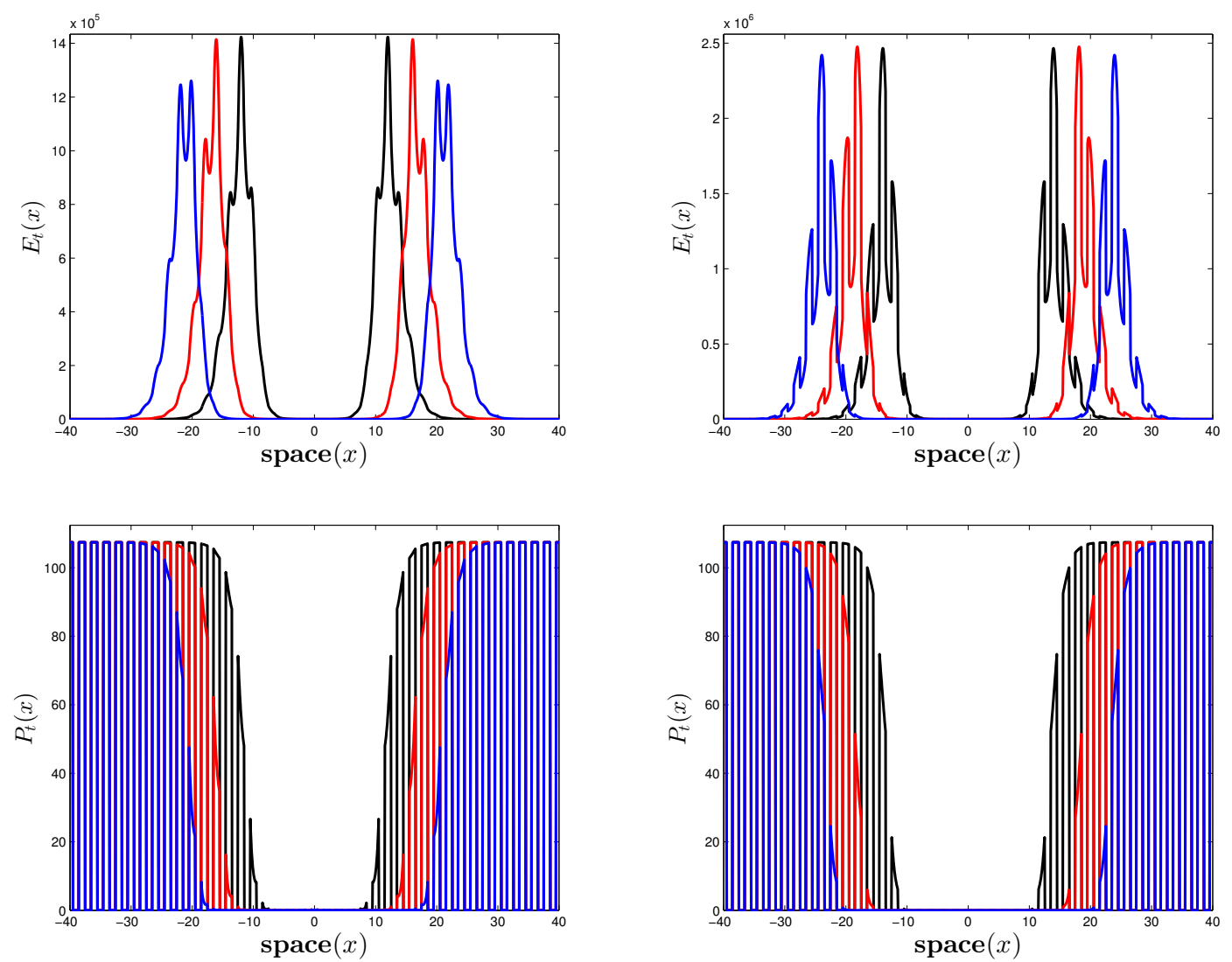

Figure 6.11: Numerical solution of system (6.4.1) for Cases C (left-hand column) and $\mathrm{M}$ (right-hand column) showing spread of EAB into a periodic landscape of host trees with uniform initial density in good patches after $t=10$ generations (black), $t=12$ generations (red) and $t=14$ generations (blue) and remaining phloem densities after $t=9$ generations (black), $t=11$ generations (red) and $t=13$ generations (blue). Parameter values were: $l_{1}=l_{2}=1, \hat{\alpha}=1, \hat{\nu}>1, z=0$, with other parameters given by the point estimates listed in Table 6.1. 


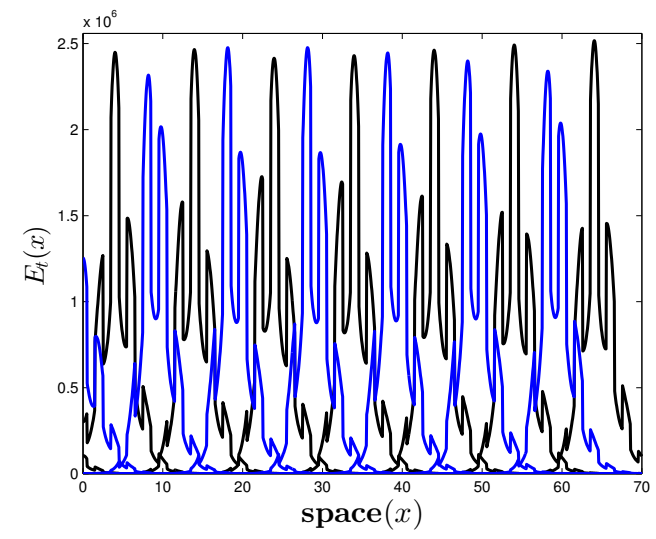

(a)

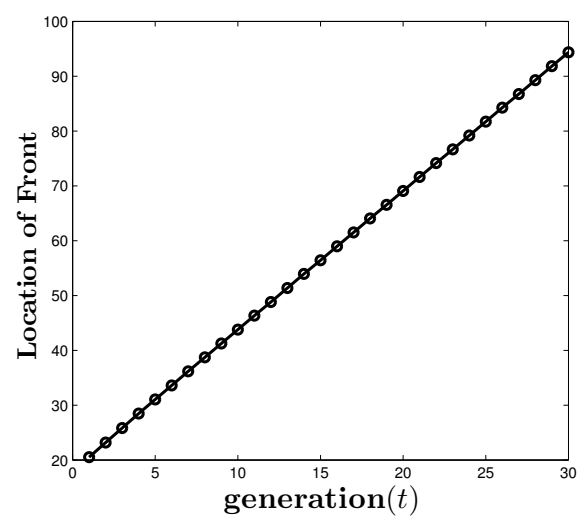

(b)

Figure 6.12: (a) Numerical simulation of system (6.2.4) for 30 generations for Case $M$ showing the spatial profile of the EAB invasion every second generation alternating between black and blue curves. The plot shows that the spatial profile of the invasion resembles that of a pulse; however, the shape of each pulse is not constant due to non-uniform phloem densities. (b) Front location of the EAB invasion showing the speed of the pulse is constant. Parameter values are given by the point estimates listed in Table 6.1 with $l_{1}=l_{2}=1, \hat{\alpha}=1, \hat{\nu}>1$, and $z=0$. 

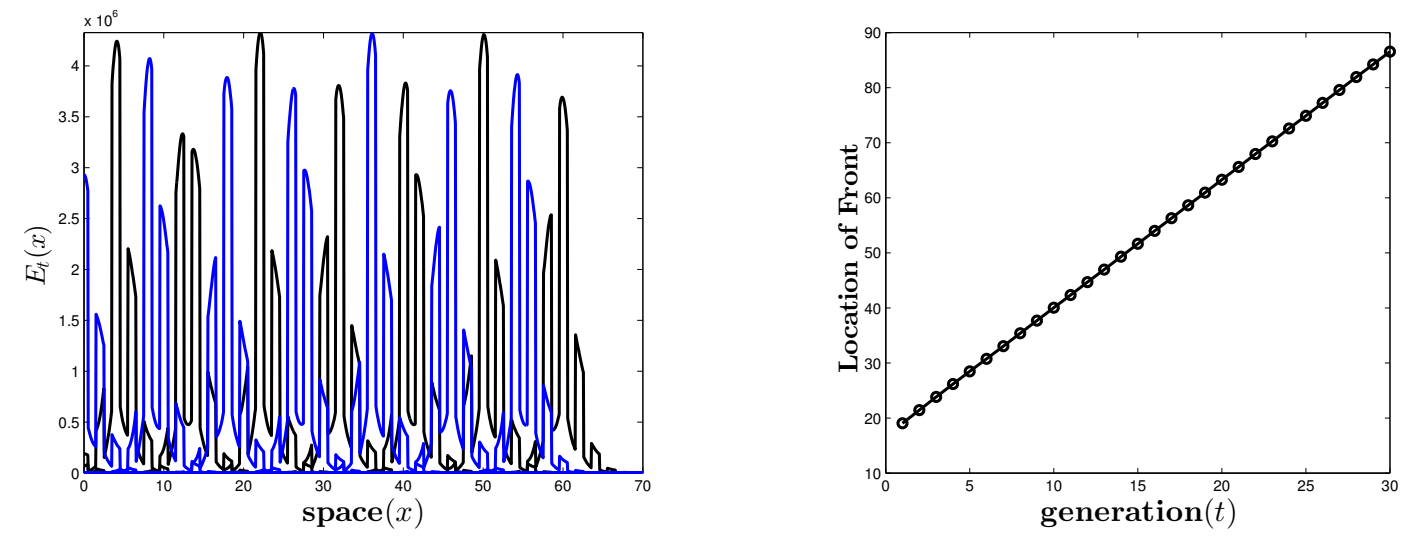

Figure 6.13: (a) Numerical simulation of system (6.2.4) for 30 generations for Case M showing the spatial profile of the EAB invasion every second generation alternating between black and blue curves, assuming individuals have a strong bias towards good patches given by $z=0.5$. The plot shows that the spatial profile of the invasion resembles that of a pulse; however, the shape of each pulse is not constant due to non-uniform phloem densities. (b) Front location of the EAB invasion showing the speed of the pulse is constant. Parameter values are given by the point estimates listed in Table 6.1 with $l_{1}=l_{2}=1, \hat{\alpha}=1$, and $\hat{\nu}>1$. 


\section{The Interplay between Individual Dispersal Mechanisms and Barrier Zones: A Case Study on the Emerald Ash Borer

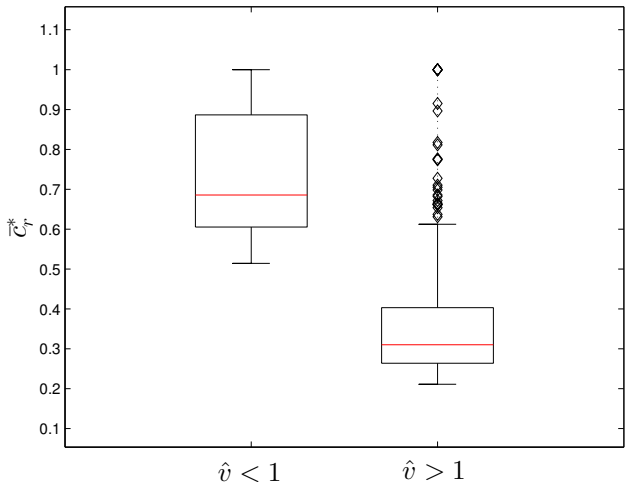

(a)

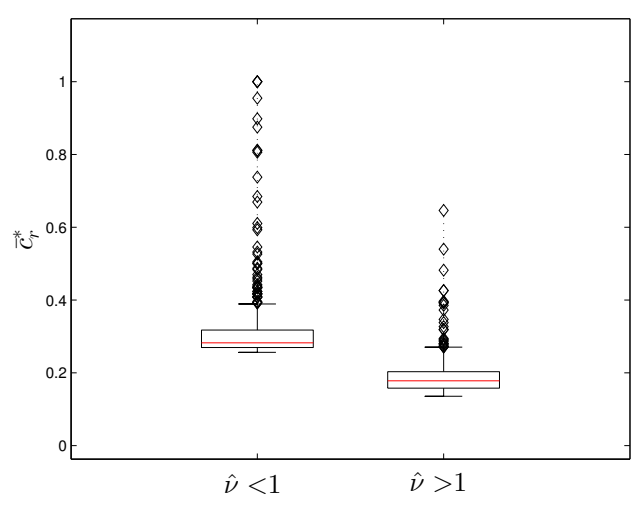

(b)

Figure 6.14: (a) Relative reduction in the spreading speed assuming barrier zones are large relative to good patches $(\hat{p}=0.1)$ (b) Relative reduction in the spreading speed assuming barrier zones and good patches are the same size $(\hat{p}=0.5)$. Non-spatial parameter values are obtained from the LHS used to generate Figure 6.4 with $\hat{\alpha}<1$ and and $z=0$.

movement behaviour at an interface for Case M.

Figure 6.14(a) shows the relative reduction in the spreading speed assuming barrier zones are large relative to good patches for the first dispersal scenario described above. Observe that, regardless of individual behavior at an interface, the spreading speed is reduced, on average, by $30 \%$ when the percentage of good habitat is reduced by $90 \%$. However, note that, if individuals move faster in bad patches, removing $90 \%$ of good habitat will not eradicate the population, and may not even result in a significant reduction in spread rates. Figure 6.14(b) shows that a 50\% reduction in good habitat results in the spreading speed being reduced, on average, by approximately $20 \%$. Also, when barrier zones and good patches are the same size, the relative reduction in the spreading speed is not sensitive to the value of $\hat{\nu}$.

The interplay between barrier zones and a movement bias at the interface is shown in Figure 6.15. If barrier zones are large relative to good patches and individual movement is biased towards bad patches, the reduction in the spreading speed is 


\section{The Interplay between Individual Dispersal Mechanisms and Barrier Zones: A Case Study on the Emerald Ash Borer

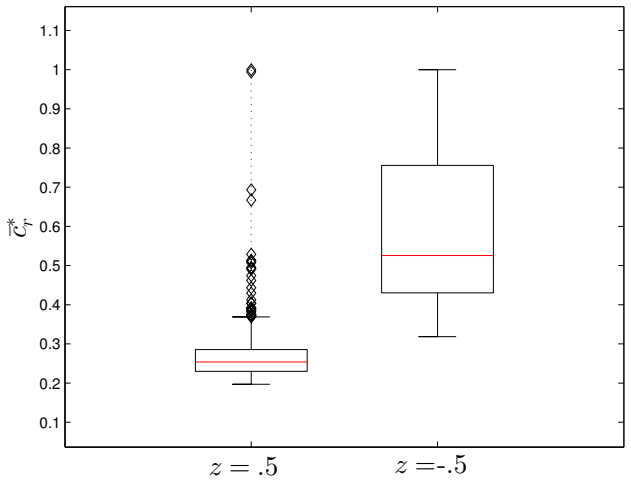

(a)

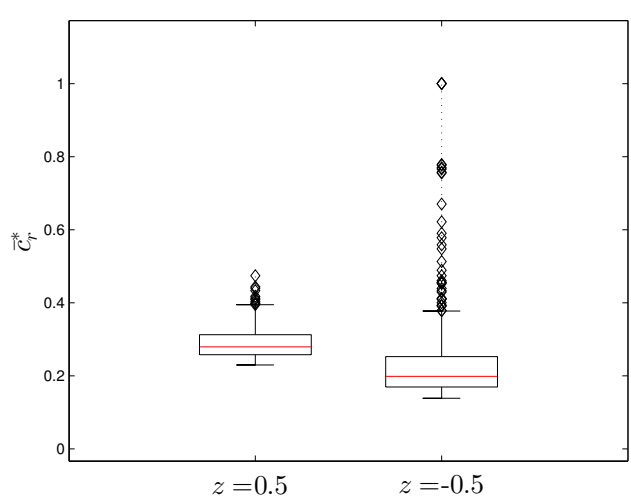

(b)

Figure 6.15: (a) Relative reduction in the spreading speed assuming barrier zones are large relative to good patches $(\hat{p}=0.1)$ (b) Relative reduction in the spreading speed assuming barrier zones and good patches are the same size $(\hat{p}=0.5)$. Non-spatial parameter values are obtained from the LHS used to generate Figure 6.4 with $\hat{\alpha}<1$ and $\hat{\nu}>1$.

significant, and may even result in a zero spread rate; see Figure 6.15(a). If the movement bias is towards good patches, the reduction is, on average, 25\%. Figure 6.15(b) shows that when barrier zones and good patches are the same size, the relative reduction in the spreading speed is not sensitive to a movement bias at an interface. Also, the average reduction is larger when the bias is towards good patches since individuals have a lower probability of leaving a good patch, resulting in a smaller spread rate. 


\section{Chapter 7}

\section{Discussion}

In this dissertation, we formulate a random-walk model in a heterogeneous landscape, and we derive the corresponding dispersal kernels. We analyze an integrodifference equation equipped with one of our kernels to study population persistence and spatial spread of an invasive forest insect in heterogeneous landscapes.

Most natural landscapes are heterogeneous on most spatial scales (Fryxell, 2008), and human activity typically introduces additional heterogeneity. Population-level studies focus on how movement between patches is affected by and in turn shapes the dynamics of a species (Bowler and Benton, 2005). Dispersal is generally described as consisting of emigration, movement to another patch and immigration, but rarely as an individual-level mechanistic movement process.

Such mechanistic movement models, in the form of correlated or uncorrelated random walks, are studied intensively from a theoretical and empirical point of view (Turchin, 1998; Schultz and Crone, 2008). These models are rarely linked to population dynamic processes. Typically, the temporal scales considered are vastly different. Experiments of random walks are conducted on the order of minutes, days or weeks, whereas reproductive time scales can be on the order of years.

In this work, we provide a framework for linking individual-level movement rules 
with population-level consequences by deriving dispersal kernels from first principles. Specifically, we demonstrate how individual-level movement rules scale up to the dispersal kernel. In Chapter 2, we begin with a mechanistic movement model and, following Ovaskainen and Cornell (2003), we explicitly include patch preference of individuals into our model. From this movement model, we generate a dispersal kernel that links population dynamics across space and between generations. Thus we follow the approach by Neubert et al. (1995) and simultaneously generalize the work by Robbins (2004) and VanKirk and Lewis (1999). To our knowledge, we are also the first to explicitly consider mortality during dispersal. The resulting dispersal kernels are in some sense related to a Laplace kernel but with some significant differences. Most importantly, the resulting kernels are typically discontinuous, and their shape depends on release location. In Chapter 3, we explore the effect of varying different parameters in a variety of scenarios, chosen mostly to match the scenarios of persistence and spread that we study in Chapters 4 and 5 .

One of the important quantities in dispersal biology is the emigration rate from a patch. We can express the probability of emigration from a patch or an assembly of patches as $1-S_{\Omega}$, where $S_{\Omega}$ is the probability of settling in that patch or assembly of patches. Hence our model gives quantitative predictions and sensitivities of the emigration rate with respect to movement rates, patch preference and other parameters. Similarly, our model makes predictions on how the emigration rate depends on matrix quality; i.e., on conditions outside the patch. We considered parameters as independent, but, in general, they could be correlated. For example, the preference for a patch could depend on the distance to the next good patch or the intrinsic growth rate of the patch.

Another crucial quantity in landscape ecology is connectivity within a network of patches, in particular for metapopulations. There are several formal definitions of connectivity, but for brief illustrative purposes here, we consider high connectivity loosely as "a sufficient number of individuals reaching any point of the network from 
any other point sufficiently fast". We suggest that the different metrics derived from the kernel can serve to explore parameter relationships and trade-offs. For example, high average dispersal success and high mean dispersal distance should correspond to high connectivity. Then overlaying the curves of average dispersal success and mean dispersal distance as a function of preference for the good patches indicates that intermediate patch preference could lead to highest connectivity. Low preference gives a low dispersal success whereas high preference decreases dispersal distance.

The work we present in Chapters 4, 5, and 6 is motivated by the spatial spread of non-native species; for example, invasive forest insects. Some of these species are highly specific in terms of their host for; example, the emerald ash borer (Agrilus planipennis). Understanding the dispersal process and its consequences for population persistence and spread is essential for designing and implementing control measures such as host tree removal and the establishment of barrier zones.

In Chapter 4, we rigorously analyze persistence conditions on a single patch and in a periodic landscape for an integrodifference equation with the dispersal kernels we derive in Chapters 2 and 3. Our work provides the analytical background to study these equations (existence of dominant eigenpair) and the explicit calculations to find the ecological quantities of interest (critical patch size, critical percentage of suitable habitat). Qualitative analysis of these results show that the behavior of these quantities depends crucially on the different interface mechanisms; i.e., on the way in which individuals respond to changes in landscape quality.

Assuming a population can persist, perhaps the most important question to consider is "at what rate will a population invade a new territory?" In Chapter 5, we consider the spreading speed problem for recursion (1.2.1) equipped with a discontinuous kernel from Chapters 2 and 3. Through numerical simulations, we show that the spatial profile of the population evolves into a discontinuous traveling periodic wave that moves with constant speed. We conjecture that linear determinacy holds, and we derive a dispersion relation for the minimal speed of the wave. Not 
surprisingly, our analysis of the dispersion relation shows that the minimal speed of the wave depends crucially on individual movement behavior at an interface.

Naturally, one may ask which set of interface conditions is most appropriate? Probably more appropriate is the question of which interface behavior is present in which species or situation. The discussion in Maciel and Lutscher (2013) gives several ecological examples that make it clear that there is no single solution for all cases. For example, Thomas (2000) found that high-mobility butterflies had the highest probability of surviving in fragmented landscapes. Assuming all other things are equal, we could conclude from Figure 4.4 that Case M would model individual movement in this scenario. We argue that, of the three interface conditions, Case $\mathrm{C}$ is probably the least likely to occur. Foraging theory suggests individuals should move more slowly in good than in bad habitat. Under the latter assumption, individuals that move according to Case $\mathrm{C}$ enter favorable habitat at a low rate, and the population will not persist for low motility in good patches (see Figure 4.4).

One of the most important quantities to consider when studying persistence and spread in fragmented landscapes is the scale of dispersal relative to the scale of landscape heterogeneity (VanKirk, 1995; With, 2002; Fahrig, 2002; Dewhirst and Lutscher, 2009). Extending VanKirk (1995), we introduce biologically relevant composite parameters to measure dispersal ability, habitat quality, and habitat heterogeneity in Section 4.5. Our results show how persistence conditions depend on the mobility of individuals within each patch type, the fragmentation level and quality of the landscape, and on the movement bias. To obtain these results, we assume that the parameters in (4.5.8) were independent of other model parameters. In general, this need not be the case; however, this assumption allows for easier analysis as it reduces the number of parameters by half.

Previously, many studies have focused on the effect of non-local dispersal on persistence (through the introduction of satellite populations), and as the driving force behind rapidly spreading invasions (Shigesada et al., 1995; Kot et al., 1996; Sharov and 
Liebhold, 1998; Bossenbroek et al., 2001). However, given the difficulty in empirically measuring long-distance dispersal events, recent efforts have focused on developing a more thorough understanding of local dispersal since effective management strategies depend on behavior locally (Mercader et al., 2009, 2011b; McCullough and Mercader, 2012). The modeling framework in this dissertation not only provides a thorough description of local dispersal, but also provides some insight into the influence that local dispersal behavior has on non-local dispersal.

In Chapter 6, we develop a strategic model for the spatial spread of EAB and interaction with host trees. In a homogeneous landscape, numerical simulations show that the spatial profile of the invasion evolves into a traveling periodic pulse that moves with constant speed. In patchy landscapes, the invasion moves at a constant speed; however, the profile of the invasion changes its shape. We conjecture that linear determinacy holds, and numerical simulations support this hypothesis. A sensitivity analysis shows that the speed of the invasion crucially depends on the average number of eggs deposited $(r)$, the overwintering survival of larvae $\left(m_{w}\right)$, and the percentage of good habitat $(\hat{p}$.)

As an application, we study the effect of barrier zones on slowing the spread of an EAB invasion. To do this, we follow Sharov and Liebhold (1998) and introduce the relative reduction in the spreading speed, given by $\bar{c}_{r}^{*}$. Our results show that there is a synergy effect between the relative width of a barrier zone and overwintering mortality of larvae, and the resulting reduction in the spreading speed. Figure 6.10(c) shows that, if a large portion of larvae survive overwintering (black dots), the relative reduction in the spreading speed is null unless at least $90 \%$ of good habitat is removed. Also, note that less than $1 \%$ of our simulations resulted in a significant reduction in the spreading speed, regardless of barrier zone width, when overwintering survival of larvae was high. As the probability that larvae survive overwintering decreases, the relative width of a barrier zone necessary to cause a significant reduction in the spreading speed also decreases; see Figure 6.10(c). 
Assuming that patch type does not affect dispersal, the results in Figure 6.10(b) show that, on average, the spreading speed is reduced by $60 \%$ when $90 \%$ of good habitat is removed, and is reduced by approximately $20 \%$ when $50 \%$ of good habitat is removed. The results for patch-dependent dispersal are much different as shown in Figures 6.14 and 6.15. For example, removing $90 \%$ of good habitat results in a reduction of, on average, 30\% when the effective motility coefficient is larger than unity. This result shows that the effectiveness of barrier zones on reducing obtained speeds may be reduced by as much as $50 \%$ when patch-dependent dispersal is included. If there is movement bias at a barrier zone towards good habitat, the reduction in the spreading speed is even less; see Figure 6.15(a). These results show that, to determine the effectiveness of barrier zones on reducing the spreading speed, it is crucial that one consider individual movement behavior at the barrier-zone interface.

A number of research topics in regards to mathematical analysis and modeling aspects arise from our work. The results of Chapter 5 open up an exciting and challenging area for mathematical analysis: namely, the existence of the spreading speed and corresponding discontinuous traveling periodic wave. Previous results on spreading speeds (Weinberger, 2002; Weinberger et al., 2008; Hsu and Zhao, 2008) required $Q$ to be a compact operator on the space of continuous functions. Operator $Q$ in (1.2.1) equipped with a discontinuous kernel is not a compact operator on the space of continuous functions.

Another challenge is to extend the results to non-monotone growth functions such as the Ricker function. Such an extension would follow previous work by Hsu and Zhao (2008); Weinberger et al. (2008). A more challenging task is to allow for an Allee effect in the growth function. Using several heuristic dispersal kernels, Dewhirst and Lutscher (2009) provided some insights in the different responses of a population to habitat fragmentation, depending on whether or not an Allee effect was present. Numerical simulations of our equation with detailed dispersal behavior included would be a first step to either confirm or contrast those results. 
We hope that the work in Chapter 6 inspires others to tackle the challenging proof for the existence of a traveling pulse solution and its properties. In a homogeneous landscape, numerical simulations show that a traveling pulse solution is formed over a wide range of parameter space and the invasion moves with constant speed. In patchy landscapes, the invasion moves with constant speed, but does not maintain its shape. In either case, the existence of a traveling pulse requires novel mathematical techniques since the system in (6.2.4) is non-monotone and non-compact.

A particularly interesting, yet challenging, modeling project is to extend the results of Chapter 2 to include the effect of perceptual range on interface conditions and obtained kernels. Zollner (2000) defines the perceptual range of a species as the maximum distance at which an individual can detect specific landscape features such as habitat boundaries. In recent years, there have been a number of empirical studies that have examined a species perceptual range in fragmented landscapes (Gillis and Nams, 1998; Zollner, 2000; Conradt et al., 2001; Schooley and Wiens, 2003). In particular, Conradt et al. (2000) showed that the perceptual range of the meadow brown butterfly, Maniola jurtina, was approximately twice that of its average dispersal distance.

Another interesting, but possibly more challenging task, is to include other movement behavior such as taxis (e.g. movement towards certain landscape features), and examine the effect of these mechanisms on interface conditions and dispersal kernels. Neubert et al. (1995) derived kernels in a homogeneous landscape from a PDE with no diffusive term, piecewise-defined taxis, and a time-dependent settling rate. The shape of obtained kernels depends on the choice of the settling rate. For example, a constant settling rate generates the Laplace kernel. More recently, Kawasaki et al. (2012) formulated a reaction-diffusion equation with a taxis term in patchy landscapes. The authors analyzed persistence conditions and derived a dispersion relation for the speed of the invasion, assuming continuous interface conditions. Interface conditions analogous to Cases $\mathrm{M}$ and $\mathrm{S}$ for a reaction-diffusion equation equipped 
with a taxis term were derived by Ovaskainen and Cornell (2003). One area of future research is to re-derive the results of Kawasaki et al. (2012) with these interface conditions. The other is to derive the corresponding dispersal kernels and analyze the resulting integrodifference equation. 


\title{
Appendix A
}

\section{Table of Variables, Parameters, Important Quantities, and Notation}

\author{
Variables \\ $\Delta t$ \\ Time step \\ $\Delta x$ \\ Spatial step size \\ $E_{t}$ \\ Density of eggs at generation $t$ \\ $N_{t}$ \\ Density of individuals at generation $t$ \\ $N^{*}$ \\ Positive steady state of equation (1.2.1) \\ $P_{t}$ \\ Density of phloem at generation $t$ \\ $t$ \\ Generation count for IDE model \\ $T$ \\ Time scale for dispersal model \\ $u$ \\ Probability density function for random walk model \\ $x$ \\ Spatial variable \\ $y$ \\ Source location
}




\section{Parameters}

$a$

$\alpha$

$\eta$

$H_{i}$

l

$l_{1}$

$l_{2}$

$\hat{l}_{i}$

$m_{w}$

$\mu_{i}$

$\nu$

$\Omega$

$p$

$\hat{p}$

$\bar{P}$

$q$

$r$

$r_{0}$

$\hat{r}$

s

z

$\zeta$
Inverse of mean dispersal distance for the Laplace kernel (1.3.3)

Spatially-dependent probability of settling

Spatially-dependent probability of dying

Consumption rate of phloem per larvae

Heterogeneity of patch type $i$ (4.5.5)

Period of the landscape

Length of a good patch

Length of a bad patch

Effective domain length of patch $i$ (4.5.2)

Over-wintering mortality of larvae

Inverse of mean dispersal distance in patch type $i$

Spatially-dependent motility coefficient

Landscape in which population resides

Spatially-dependent movement probability

Measure of landscape fragmentation

Boundary permeability

Quality of the habitat (4.5.4)

Average number of eggs laid per adult female

Reproductive rate for growth function $f ; r_{0}=\left.\frac{\partial f}{\partial N}\right|_{N=0}$

Reproductive rate of settled individuals

Shape parameter for a travelling wave

Measure of the degree of bias at an interface

Parameter in the phloem-dependent failure rate for larval development 


\section{Important Quantites}

$c$

$c^{*}$

$\bar{c}^{*}$

$\bar{c}_{r}^{*}$

$l^{*}$

$l_{\{m, s, c\}}^{*}$

$l_{1,\{M, S, C\}}^{*}$

\section{Notation}

\section{$B$}

$\delta$

$f$

$g$

$h$

$k$

$\mathcal{L}$

$\mathbb{L}_{+}^{\infty}(\mathbb{R})$

$\mathbb{L}_{l,+}^{\infty}(\mathbb{R})$

$\mathbb{L}_{+}^{\infty}([0, l])$

$\mathbb{L}_{+}^{2}([0, l])$

$M$

$\phi$

$Q$

$\tilde{Q}$

$Q_{l}$
Minimal speed of a traveling wave

Asymptotic spreading speed

Average frontal speed

Relative reduction in the spreading speed caused by a barrier zone

Critical domain size in a homogeneous landscape

Critical domain size for Cases M (4.1.17), S (4.1.18), and C (4.1.19)

Critical size of good patches for Cases M (4.4.11), and S/C (4.4.12)

Linearization of $Q$

Dirac-delta distribution

Nonlinear growth function for IDE

Nonlinear function to model EAB egg deposition

Nonlinear function to model consumption of phloem by EAB

Dispersal kernel

$k$ is the Green's function of the Linear differential operator $\mathcal{L}$

The set of nonnegative, essentially bounded functions on $\mathbb{R}$

The restriction of $\mathbb{L}_{+}^{\infty}(\mathbb{R})$ to $l$-periodic functions

The restriction of $\mathbb{L}_{l,+}^{\infty}(\mathbb{R})$ to one period

The set of nonnegative, square-integrable functions on $[0, l]$

Moment-generating function of the dispersal kernel

Nonnegative eigenfunction

Integral operator representation for IDE

Convolution operator representation for an IDE

Restriction of $Q$ to $\mathbb{L}_{l,+}^{\infty}(\mathbb{R})$ 


$\begin{array}{ll}\hat{Q}_{l} & \text { Restriction of } Q_{l} \text { to } \mathbb{L}_{+}^{\infty}([0, l]) \\ \hat{s} & \text { Dispersal success function } \\ S & \text { Average dispersal success }\end{array}$




\section{Appendix B}

\section{Interchange of Limit and Integral Operator}

Recall the general dispersal model on a landscape comprised of two patch types, described in Section 2.1, is given by

$$
\frac{\partial u(x, T ; y)}{\partial T}=\left\{\begin{array}{l}
\nu_{1} \frac{\partial^{2} u(x, T ; y)}{\partial x^{2}}-\left(\alpha_{1}+\beta_{1}\right) u(x, T ; y), x>a \\
\nu_{2} \frac{\partial^{2} u(x, T ; y)}{\partial x^{2}}-\left(\alpha_{2}+\beta_{2}\right) u(x, T ; y), x<a
\end{array}\right.
$$

We assume that the interface conditions are given by Case M (2.3.3), we choose $a=0$, and $y>0$.

By applying the method of images (see Chapter 1 in Kevorkian (1990)), the solution to (B.0.1) is given by

$$
u(x, T ; y)=\left\{\begin{array}{l}
e^{-\left(\alpha_{2}+\beta_{2}\right) T} u_{2}(x, T ; y), x<0 \\
e^{-\left(\alpha_{1}+\beta_{1}\right) T} u_{1}(x, T ; y), x>0
\end{array}\right.
$$


where

$$
\begin{aligned}
& u_{2}(x, T ; y)=\frac{e^{-\frac{\left(x-\sqrt{\frac{\nu_{2}}{\nu_{1}}} y\right)^{2}}{4 \nu_{2} T}}}{\left(1+\frac{1+z}{1-z} \sqrt{\frac{\nu_{2}}{\nu_{1}}}\right) \sqrt{\pi \nu_{2} T}} \\
& u_{1}(x, T ; y)=\frac{e^{-\frac{(x-y)^{2}}{4 \nu_{1} T}}+\eta e^{-\frac{(x+y)^{2}}{4 \nu_{1} T}}}{2 \sqrt{\pi \nu_{1} T}},
\end{aligned}
$$

with

$$
\eta=\frac{\frac{1+z}{1-z} \sqrt{\frac{\nu_{2}}{\nu_{1}}}-1}{\frac{1+z}{1-z} \sqrt{\frac{\nu_{2}}{\nu_{1}}}+1}
$$

Function $u_{2}(x, T ; y)$ is monotone increasing in $x$ for all $x<\sqrt{\frac{\nu_{2}}{\nu_{1}}} y$ and hence for all $x<0$. To verify that $u_{1}(x, T ; y)$ is monotone increasing in $x$ for $0<x<y$, we differentiate and obtain

$$
\frac{\partial u}{\partial x}=\frac{\frac{-2(x-y)}{4 \nu_{1} T} e^{-\frac{(x-y)^{2}}{4 \nu_{1} T}}-\frac{2(x+y) \eta}{4 \nu_{1} T} e^{-\frac{(x+y)^{2}}{4 \nu_{1} T}}}{2 \sqrt{\pi \nu_{1} T}} .
$$

It is only necessary for $u_{1}(x, T ; y)$ to be monotone increasing near $x=0$. Thus, setting $x=0$ in (B.0.4) and simplifying, we obtain

$$
\frac{\partial u}{\partial x}=\frac{y e^{-\frac{y^{2}}{4 \nu_{1} T}}}{4 \nu_{1} T \sqrt{\pi \nu_{1} T}}(1-\eta)
$$

Since $|\eta|<1, u_{1}(x, T ; y)$ is a monotone increasing function in $x$ for $x$ near zero.

We define the sequences $\phi_{n}^{1,2}(T)$ as

$$
\lim _{n \rightarrow \infty} \phi_{n}^{1,2}(T)=\lim _{n \rightarrow \infty} u( \pm 1 / n, T ; y)=\lim _{x \rightarrow 0^{ \pm}} u(x, T ; y)
$$

Sequences $\phi_{n}^{1}(T)$ and $\phi_{n}^{2}(T)$ are monotone decreasing and increasing, respectively. Therefore, since the sequence $\psi_{n}^{1}(T)=\phi_{1}^{1}(T)-\phi_{n}^{1}(T)$ is monotone increasing, the 
interchange of the limit and integral operator in (2.4.2) and (2.4.4) is justified by applying the monotone convergence theorem.

Since (B.0.1) is translation invariant with respect to $a$, the above result holds for all $a$ with $y>a$. If $y<a$ (i.e. release point in the bad patch), $u_{2}(x, T ; y)$ in (B.0.2) is a monotone decreasing sequence for all $x>-\sqrt{\frac{\nu_{2}}{\nu_{1}}} y$, and $u_{1}(x, T ; y)$ in (B.0.2) is a monotone decreasing sequence for all $x>a$. Therefore, by an argument analogous to above, the monotone convergence theorem applies. 


\section{Appendix C}

\section{Derivation of Interface Conditions}

Ovaskainen and Cornell (2003) and Maciel and Lutscher (2013) studied a random walk model in a patchy landscape, and assumed that at the interface of two patch types individuals exhibit patch-preference movement behavior. In general, this assumption leads to the probability density function of the random walker being discontinuous at an interface. In this appendix, we follow previous authors and give a derivation of interface conditions (2.3.3) - (2.3.5).

We model the movement of individuals as a random walk in a patchy landscape comprised of two patch types: good habitat and unfavorable matrix. Within each patch type, model parameters are constant so that parameter functions are piecewise

constant (see equation (2.3.1)). Within each patch the standard approach is to derive a diffusion equation from a master equation by taking the parabolic limit (Turchin, 1998). Here, we focus on the interface condition. For simplicity, we assume that the interface between the two patch types is $x=0$.

During each time step of length $\Delta t$, individuals may or may not move. Within patch type $i$, the probability that an individual moves is given by $p_{i} \Delta t$. We assume 
that there is no bias in movement direction. At an interface, an individual moves to the left with probability $p_{0}^{-} \Delta t$, to the right with probability $p_{0}^{+} \Delta t$, and does not move with probability $\left(1-\left(p_{0}^{-}+p_{0}^{+}\right) \Delta t\right)$. Each step within patch type $i$ is of length $\Delta x_{i}$ to the left or right with equal probability. Furthermore, the probabilities of dying and settling during each time step in patch $i$ are $\beta_{i} \Delta t$ and $\alpha_{i} \Delta t$, respectively. At an interface, the probabilities are given by $\beta_{0} \Delta t$ and $\alpha_{0} \Delta t$, respectively. For ease of notation below, we let $c_{i}=\alpha_{i}+\beta_{i}$.

Let $u(x, T ; y)$ denote the probability per unit length of finding an individual at some location $x$ at some time $T$, assuming the individual emerged from some location $y$ at time zero. Then, we have the following master equations for the density function near the interface $u\left(-\Delta x_{2}, T ; y\right), u(0, T ; y)$, and $u\left(\Delta x_{1}, T ; y\right)$ :

$$
\begin{aligned}
& \Delta x_{2} u\left(-\Delta x_{2}, T+\Delta t ; y\right)=\left(1-c_{2} \Delta t\right)\left(1-p_{2} \Delta t\right) \Delta x_{2} u\left(-\Delta x_{2}, T ; y\right) \\
& +\left(1-c_{2} \Delta t\right)\left(\frac{p_{2}}{2} \Delta t \Delta x_{2} u\left(-2 \Delta x_{2}, T ; y\right)+p_{0}^{-} \Delta t \Delta x_{0} u(0, T ; y)\right) \\
& \Delta x_{0} u(0, T+\Delta t ; y)=\left(1-c_{0} \Delta t\right)\left(1-\left(p_{0}^{-}+p_{0}^{+}\right) \Delta t\right) \Delta x_{0} u(0, T ; y) \\
& +\left(1-c_{0} \Delta t\right)\left(\frac{p_{2}}{2} \Delta t \Delta x_{2} u\left(-\Delta x_{2}, T ; y\right)+\frac{p_{1}}{2} \Delta x_{1} u\left(\Delta x_{1}, T ; y\right)\right) \\
& \Delta x_{1} u\left(\Delta x_{1}, T+\Delta t ; y\right)=\left(1-c_{1} \Delta t\right)\left(1-p_{1} \Delta t\right) \Delta x_{1} u\left(\Delta x_{1}, T ; y\right) \\
& +\left(1-c_{1} \Delta t\right)\left(\frac{p_{1}}{2} \Delta t \Delta x_{1} u\left(2 \Delta x_{1}, T ; y\right)+p_{0}^{+} \Delta x_{0} u(0, T ; y)\right) .
\end{aligned}
$$

Now, expanding terms containing $2 \Delta x_{i}$ in a Taylor series about $\Delta x_{i}$ and rearranging, 
we obtain

$$
\begin{aligned}
& \frac{\Delta x_{2}}{\Delta t}\left(u\left(-\Delta x_{2}, T+\Delta t ; y\right)-u\left(-\Delta x_{2}, T ; y\right)\right)+\left(c_{2}+\frac{p_{2}}{2}\right) \Delta x_{2} u\left(-\Delta x_{2}, T ; y\right) \\
& =p_{0}^{-} \Delta x_{0} u(0, T ; y)+\mathcal{O}(\Delta t)+\mathcal{O}\left(\left(\Delta x_{2}\right)^{2}\right)+\ldots \\
& \frac{\Delta x_{0}}{\Delta t}(u(0, T+\Delta t ; y)-u(0, T ; y))+\left(c_{0}+p_{0}^{-}+p_{0}^{+}\right) \Delta x_{0} u(0, T ; y) \\
& =\frac{p_{2}}{2} \Delta x_{2} u\left(-\Delta x_{2}, T ; y\right)+\frac{p_{1}}{2} \Delta x_{1} u\left(\Delta x_{1}, T ; y\right)+\mathcal{O}(\Delta t)+\mathcal{O}\left(\left(\Delta x_{0}\right)^{2}\right)+\ldots
\end{aligned}
$$

$$
\begin{aligned}
& \frac{\Delta x_{1}}{\Delta t}\left(u\left(\Delta x_{1}, T+\Delta t ; y\right)-u\left(\Delta x_{1}, T ; y\right)\right)+\left(c_{1}+\frac{p_{1}}{2}\right) \Delta x_{1} u\left(\Delta x_{1}, T ; y\right) \\
& =p_{0}^{+} \Delta x_{0} u(0, t ; y)+\mathcal{O}(\Delta t)+\mathcal{O}\left(\left(\Delta x_{1}\right)^{2}\right)+\ldots
\end{aligned}
$$

Multiplying the first equation in (C.0.2) by $p_{0}^{+}$, the third equation by $p_{0}^{-}$and subtracting, we obtain

$$
\begin{aligned}
& p_{0}^{-} \frac{\Delta x_{1}}{\Delta t}\left(u\left(\Delta x_{1}, T+\Delta t ; y\right)-u\left(\Delta x_{1}, T ; y\right)\right)+p_{0}^{-}\left(c_{1}+\frac{p_{1}}{2}\right) \Delta x_{1} u\left(\Delta x_{1}, T ; y\right) \\
& =p_{0}^{+} \frac{\Delta x_{2}}{\Delta t}\left(u\left(-\Delta x_{2}, T+\Delta t ; y\right)-u\left(-\Delta x_{2}, T ; y\right)\right)+p_{0}^{+}\left(c_{2}+\frac{p_{2}}{2}\right) \Delta x_{2} u\left(-\Delta x_{2}, T ; y\right) \\
& +\mathcal{O}(\Delta t)+\mathcal{O}\left(\left(\Delta x_{1}\right)^{2}\right)+\mathcal{O}\left(\left(\Delta x_{2}\right)^{2}\right)+\ldots
\end{aligned}
$$

Now, expanding $u(x, T ; y)$ in a Taylor series about $\Delta t$ we obtain the relations

$$
\begin{aligned}
& u\left(-\Delta x_{2}, T+\Delta t ; y\right)=u\left(-\Delta x_{2}, T ; y\right)+\Delta t \frac{\partial u\left(-\Delta x_{2}, T ; y\right)}{\partial T}+\mathcal{O}\left((\Delta t)^{2}\right) \\
& u\left(\Delta x_{1}, T+\Delta t ; y\right)=u\left(\Delta x_{1}, T ; y\right)+\Delta t \frac{\partial u\left(\Delta x_{1}, T ; y\right)}{\partial T}+\mathcal{O}\left((\Delta t)^{2}\right) .
\end{aligned}
$$

Multiplying the first equation in (C.0.4) by $\Delta x_{2} p_{0}^{+}\left(1-\left(c_{2}+p_{2}\right) \Delta t\right)$, the second by 
$\Delta x_{1} p_{0}^{-}\left(1-\left(c_{1}+p_{1} \Delta t\right)\right.$, and subtracting the resulting expressions we obtain

$$
\begin{aligned}
& p_{0}^{-} \frac{\Delta x_{1}}{\Delta t}\left(u\left(\Delta x_{1}, T+\Delta t ; y\right)-u\left(\Delta x_{1}, T ; y\right)\right)+p_{0}^{-}\left(c_{1}+p_{1}\right) \Delta x_{1} u\left(\Delta x_{1}, T ; y\right) \\
& =p_{0}^{+} \frac{\Delta x_{2}}{\Delta t}\left(u\left(-\Delta x_{2}, T+\Delta t ; y\right)-u\left(-\Delta x_{2}, T ; y\right)\right)+p_{0}^{+}\left(c_{2}+p_{2}\right) \Delta x_{2} u\left(-\Delta x_{2}, T ; y\right) \\
& +\mathcal{O}(\Delta t)+\mathcal{O}\left(\left(\Delta x_{1}\right)^{2}\right)+\mathcal{O}\left(\left(\Delta x_{2}\right)^{2}\right)+\ldots
\end{aligned}
$$

Subtracting equation (C.0.5) from (C.0.3) and taking the parabolic limit as $\Delta x_{i}$, $\Delta t \rightarrow 0$, we obtain

$$
p_{0}^{+} p_{2} \lim _{\Delta x_{i} \rightarrow 0}\left(\frac{\Delta x_{2}}{\Delta x_{1}}\right) u\left(0^{-}, T ; y\right)=p_{0}^{-} p_{1} u\left(0^{+}, T ; y\right)
$$

where $0^{ \pm}$is short for the limit as $x$ approaches 0 from above and below, respectively. To obtain the first condition for Case C (see (2.3.5)), we assume $\Delta x_{1}=\Delta x_{2}, p_{0}^{-}=$ $p_{2} / 2$, and $p_{0}^{+}=p_{1} / 2$ in (C.0.6) and obtain

$$
u\left(0^{-}, T ; y\right)=u\left(0^{+}, T ; y\right)
$$

Therefore, continuous interface conditions arise when within patch movement rules are the same as movement rules at an interface. Recalling the definition of the motility coefficient in (2.1.2), assuming $\Delta x_{1}=\Delta x_{2}$ and defining

$$
p_{0}^{-}=\frac{1-z}{2}, \quad p_{0}^{+}=\frac{1+z}{2},
$$

we obtain the first condition in Case M (see (2.3.3)). The first condition in Case S (see (2.3.4)) follows by assuming $p_{1}=p_{2}$.

To derive the continuity of the flux condition, we begin with the following relation 
between the fluxes

$$
\begin{aligned}
& \nu_{1} \frac{\partial u\left(\Delta x_{1}, T ; y\right)}{\partial x}-\nu_{2} \frac{\partial u\left(-\Delta x_{2}, T ; y\right)}{\partial x} \\
& =\nu_{1} \frac{u\left(2 \Delta x_{1}, T ; y\right)-u\left(\Delta x_{1}, T ; y\right)}{\Delta x_{1}}-\nu_{2} \frac{u\left(-\Delta x_{2}, T ; y\right)-u\left(-2 \Delta x_{2}, T ; y\right)}{\Delta x_{2}}
\end{aligned}
$$

Now, Taylor expanding the equations in (C.0.1) for small $\Delta t$, we obtain

$$
\begin{aligned}
& u\left(-2 \Delta x_{2}, T ; y\right)-u\left(-\Delta x_{2}, T ; y\right)=u\left(-\Delta x_{2}, T ; y\right)-\frac{2 p_{0}^{-} \Delta x_{0}}{p_{2} \Delta x_{2}} u(0, T ; y) \\
& +\frac{2}{p_{2}} \frac{\partial u\left(-\Delta x_{2}, T ; y\right)}{\partial T}+\mathcal{O}\left((\Delta t)^{2}\right) \\
& \left(p_{0}^{-}+p_{0}^{+}\right) \Delta x_{0} u(0, T ; y)=\frac{p_{1}}{2} \Delta x_{1} u\left(\Delta x_{1}, T ; y\right)+\frac{p_{2}}{2} \Delta x_{2} u\left(-\Delta x_{2}, T ; y\right) \\
& +\Delta x_{0} \frac{\partial u(0, T ; y)}{\partial T}+\mathcal{O}\left((\Delta t)^{2}\right) \\
& u\left(2 \Delta x_{1}, T ; y\right)-u\left(\Delta x_{1}, T ; y\right)=u\left(\Delta x_{1}, T ; y\right)-\frac{2 p_{0}^{+} \Delta x_{0}}{p_{1} \Delta x_{1}} u(0, T ; y) \\
& +\frac{2}{p_{1}} \frac{\partial u\left(\Delta x_{1}, T ; y\right)}{\partial T}+\mathcal{O}\left((\Delta t)^{2}\right) .
\end{aligned}
$$

Substituting the relations in (C.0.8) into (C.0.7) and recalling the definition of the motility coefficient in (2.1.2), we obtain

$$
\begin{aligned}
& \nu_{1} \frac{\partial u\left(\Delta x_{1}, T ; y\right)}{\partial x}-\nu_{2} \frac{\partial u\left(\Delta x_{2}, T ; y\right)}{\partial x} \\
& =\frac{-2}{\Delta t}\left(\Delta x_{1} \frac{\partial u\left(\Delta x_{1}, T ; y\right)}{\partial T}+\Delta x_{2} \frac{\partial u\left(\Delta x_{2}, T ; y\right)}{\partial T}+\Delta x_{0} \frac{\partial u(\Delta 0, T ; y)}{\partial T}\right)+\mathcal{O}\left((\Delta t)^{2}\right)+\ldots
\end{aligned}
$$

Taking the parabolic limit as $\Delta x_{i}, \Delta t \rightarrow 0$ in such a way that $\Delta t=\mathcal{O}\left(\Delta x_{i}\right)$, we 
obtain the continuity of the flux condition given by

$$
\nu_{1} \frac{\partial u\left(0^{+}, T ; y\right)}{\partial x}=\nu_{2} \frac{\partial u\left(0^{-}, T ; y\right)}{\partial x} .
$$




\section{Appendix D}

\section{Existence of Dominant Eigenvalue for the Critical Domain Size Problem}

Brown et al. (1990) proved the existence of a dominant eigenvalue and unique (up to constant multiple) nonnegative eigenfunction for the following weighted eigenvalue problem

$$
-\Delta u(x)=\lambda g(x) u, u \rightarrow 0 \text { as } x \rightarrow \infty
$$

under the additional assumption that $g(x)$ is negative for sufficiently large $|x|$. The system of ODEs given by (4.1.5) can be embedded into the framework of Brown et al. by rewriting the system as

$$
-\frac{d^{2}}{d x^{2}} \phi(x)=\mu g(x, \omega) \phi(x),
$$




\section{Existence of Dominant Eigenvalue for the Critical Domain Size Problem159}

where

$$
g(x, \omega)=\left\{\begin{array}{r}
\mu_{1}\left[\omega \hat{r}_{1}-1\right] \phi(x),|x|<l / 2 \\
-\mu_{2}\left[1-\omega \hat{r}_{2}\right] \phi(x),|x|>l / 2 .
\end{array}\right.
$$

By the results of Brown et al. (1990), there exists a unique $\mu(\omega)=\mu(\lambda)$ corresponding to a nonnegative eigenfunction for (D.0.2). Furthermore, $\mu(\lambda)=1$ corresponds to a positive solution for (4.1.5). Therefore, if we can show that (4.1.5) possess a positive solution, then we must have $\mu(\lambda)=1$ in (D.0.2) by the uniqueness of $\mu$.

In Section 4.1, we showed that the solution to (4.1.5) is given by

$$
\phi(x)=\left\{\begin{array}{l}
A \exp \left(-\sqrt{\mu_{2}\left(1-\omega \hat{r}_{2}\right.} x\right), x \geq l / 2 \\
\cos \left(\sqrt{\mu_{1}\left(\frac{\hat{r}_{1}}{\lambda}-1\right)} x\right), 0 \leq x \leq l / 2 .
\end{array}\right.
$$

It is obvious that $\phi$ is positive when $x \geq l / 2$. To show that $\phi$ is positive for $0 \leq x \leq$ $l / 2$, we must show that

$$
0 \leq \sqrt{\mu_{1}\left(\frac{\hat{r}_{1}}{\lambda}-1\right)} x \leq \frac{\pi}{2},
$$

where $\lambda \in\left(\max \left\{\lambda^{*}, \hat{r}_{2}\right\}, \hat{r}_{1}\right)$ and $\lambda^{*}$ satisfies

$$
\sqrt{\mu_{1}\left(\frac{\hat{r}_{1}}{\lambda^{*}}-1\right)} \frac{l}{2}=\frac{\pi}{2} .
$$

Now, if $x=0$, then (D.0.6) is trivially satisfied. For $x=l / 2$, we obtain

$$
\sqrt{\mu_{1}\left(\frac{\hat{r}_{1}}{\lambda}-1\right)} \frac{l}{2} \leq \frac{\pi}{2} \Rightarrow \lambda \geq \hat{r}_{1} \frac{\mu_{1} l^{2}}{\mu_{1} l^{2}+\pi^{2}}
$$




\section{Existence of Dominant Eigenvalue for the Critical Domain Size Problem160}

Solving (D.0.6) for $\lambda^{*}$, one obtains

$$
\lambda^{*}=\hat{r}_{1} \frac{\mu_{1} l^{2}}{\mu_{1} l^{2}+\pi^{2}} .
$$

Therefore, (D.0.7) is satisfied for all $\lambda \in\left(\max \left\{\lambda^{*}, \hat{r}_{2}\right\}, \hat{r}_{1}\right)$ and, therefore, (D.0.5) is satisfied showing the cosine function in (D.0.4) is positive. 


\section{Appendix E}

\section{Results on Integral Operators}

By largely following the analysis of VanKirk (1995) and employing the results of Krasnosel'skii (1964a) and Krasnosel'skii and Zabresiko (1984), we verify the technical requirements listed in properties 4.2.5 and 4.2.6 to prove the existence of a simple,

dominant, positive eigenvalue and corresponding nonnegative eigenfunction for $\widehat{Q}_{l}$ given by

$$
\widehat{Q}_{l}[N(x)]:=\int_{0}^{l} f(N(y) ; y) \widehat{k}(x, y) d y .
$$

We then show that equation (E.0.1) possess a unique, positive fixed point that is globally stable when the dominant eigenvalue of its linearization, given by

$$
B[N](x):=\int_{0}^{l} r_{0}(y) N(y) \widehat{k}(x, y) d y,
$$

is larger than unity.

Some of these results require that the function

$$
H(x, y, N)=\frac{g(x, y, N)}{N}=\frac{\widehat{k}(x, y) f(N(y) ; y)}{N}
$$


decreases as $N$ increases and is nonnegative. Replacing $f$ in (E.0.3) with the BevertonHolt function (1.2.5) we obtain

$$
H(x, y, N)=\widehat{k}(x, y) \frac{r_{0}}{1+\left(r_{0}-1\right) N},
$$

which clearly decreases as $N$ increases and is nonnegative since $\widehat{k}$ is.

We re-state Properties 4.2.5 and 4.2.6 for convenience of the reader.

Property E.0.1 The nonlinear operator $\widehat{Q}_{l}: \mathbb{L}_{+}^{2}(\Omega) \rightarrow \mathbb{L}_{+}^{2}(\Omega)$ as defined by (4.2.5) is completely continuous, Fréchet differentiable with respect to $\mathbb{L}_{+}^{2}(\Omega)$ at $N^{*}(x)=0$, and its Fréchet derivative is the completely continuous operator given by

$$
B[N](x):=\int_{0}^{l} r_{0}(y) N(y) \widehat{k}(x, y) d y, \quad r_{0}(y)=\frac{\partial f}{\partial N}(0 ; y) .
$$

Property E.0.2 Under the assumptions of this section, the linear operator $B$ has a positive, simple eigenvalue $\lambda$ corresponding to a nonnegative eigenfunction $\phi(x)$. This eigenvalue is strictly larger in modulus than any other eigenvalue of $B$, and its eigenfunction is the only (up to a constant multiple) nonnegative eigenfunction of $B$.

Compactness of $\widehat{Q}_{l}$ follows from Theorem 7.1 on page 233 of Krasnosel'skii (1964a) which is stated below for convenience of the reader.

Theorem E.0.3 (Theorem 7.1, page 233 in Krasnosel'skii (1964a)) Suppose that

$$
|w(x, y, N(y))| \leq M(x, y)\left(a+b|N|^{\alpha}\right)
$$


for some $\alpha \geq 0$,

$$
\int_{\Omega \times \Omega}|M(x, y)|^{\beta} d x d y<\infty
$$

for some $\beta>1$, and $w$ is continuous with respect to $N$. If $\alpha \leq \beta-1$, then the nonlinear operator

$$
\mathcal{B} \phi \equiv \int_{\Omega} w(x, y, \phi(y)) d y
$$

acts and is completely continuous in $L^{p}(\Omega)$ for all $p>1$ such that

$$
\frac{\alpha \beta}{\beta-1} \leq p \leq \beta
$$

To apply this theorem, we note the Beverton-Holt function (1.2.5) is bounded above by $\frac{r_{0}}{r_{0}-1}$. Since Beverton-Holt function is differentiable for all $N$, it is also continuous, and thus $f$ is continuous with respect to $N$. Furthermore, Lemma 4.2.4 showed that $\widehat{k}(x, y)$ is essentially bounded on $\Omega \times \Omega$ and therefore lies in $L^{2}(\Omega \times \Omega)$. Thus, taking $M(x, y)=\widehat{k}(x, y)$, the above theorem applies with $\alpha=1$ and $\beta=2$.

To verify that $\widehat{Q}_{l}$ is Fréchet differentiable at zero we apply Theorem 7.3 on page 238 from Krasnosel'skii (1964a).

Theorem E.0.4 ( $\widehat{Q}_{l}$ is Fréchet differentiable) Let the function $H(x, y, N)$ be nonnegative and decreasing as $N$ increases, and let $g(x, y, 0)=0$. Then, if the following derivative exists

$$
\frac{\partial g(x, y, 0)}{\partial N}=R(x, y) \text { and } \int_{\Omega} \int_{\Omega}|R(x, y)|^{q} d x d y<\infty, q>1
$$


the Fréchet derivative of $\widehat{Q}_{l}$ exists and is given by

$$
B[N](x)=\int_{\Omega} \widehat{k}(x, y) \frac{\partial f}{\partial N}(0 ; y) N(y) d y
$$

First, we note that $g(x, y, 0)=\widehat{k}(x, y) f(0, y)=0$ and

$$
\frac{\partial g(x, y, 0)}{\partial N}=\widehat{k}(x, y) r_{0}(y):=R(x, y)
$$

Therefore, since $\widehat{k}$ is square integrable and $r_{0}$ is bounded, Theorem (E.0.4) applies with $q=2$ and $\widehat{Q}_{l}$ is Fréchet differentiable at zero. The compactness of $B$ follows from Theorem 17.1 in Krasnosel'skii and Zabresiko (1984) which states that if an operator is compact and Fréchet differentiable at some point then its derivative is a compact linear operator.

To verify the statements in Property E.0.2 requires a definition of positivity for operator $B$ in the underlying space. Positivity is obtained through the notion of a cone which is defined as follows.

Definition E.0.5 (A cone in a Banach space) Let $X$ be a real Banach space with the zero element $\theta$. A subset $P \subset X$ is called a cone, if the following conditions are met:

a. $u \in P, v \in P$ imply $\alpha u+\beta v \in P$ for nonnegative scalars $\alpha \geq 0, \beta \geq 0$;

b. $u \in P,-u \in P$ imply $u=\theta$;

c. $P$ is closed. 
In particular, we see that a cone is a closed, convex set. To apply the theorems of Krasnosel'skii and Krein-Rutman requires the cone be a reproducing cone, which is defined as follows.

Definition E.0.6 (A reproducing cone) A cone, $P \subset X$, is called reproducing if every $x \in X$ can be written in the form $x=u-v$ with $u, v \in P$.

The existence of at least one eigenvalue and corresponding eigenfunction for $B$ follows from a theorem of Krein and Rutman (Krein and Rutman, 1950), which is stated as Theorem 2.5 and proved in Krasnosel'skii (1964a).

Theorem E.0.7 (Krein-Rutman) Suppose that A is a compact linear operator that leaves the positive, reproducing cone invariant, i.e. $A P \subset P$. Let the relation

$$
A^{p} u \geq \chi u, \quad \chi>0,
$$

be satisfied for some non-zero element $u \in P$ and some natural number $p$. Then the operator A has at least one characteristic vector (eigenvector) $\phi_{0}$ in P:

$$
A \phi_{0}=\lambda_{0} \phi_{0},\left(\lambda_{0}>0, \phi_{0} \in P, \phi_{0} \neq 0\right)
$$

where the positive characteristic number (eigenvalue) $\lambda_{0}$ satisfies

$$
\lambda_{0} \geq \sqrt[p]{\chi}
$$

We apply Theorem E.0.7 for the compact linear operator $B$ given by (E.0.2) and any non-zero $\phi \in P$. Since $f$ is bounded and $\widehat{k}$ is positive as a result of Lemma 4.2.4, 
$B$ leaves the cone of nonnegative $\mathbb{L}^{2}$ functions invariant. Furthermore, Krasnosel'skii proved that the cone of nonnegative $\mathbb{L}^{2}$ functions is reproducing. By assumption F5 in Chapter $1, r_{0}(x)$ is strictly larger than unity for all $x$ so there exists an $\hat{r}$ such that

$$
B[\phi]=\int_{\Omega} \widehat{k}(x, y) r_{0}(y) \phi(y) d y \geq \hat{r} \int_{\Omega} \widehat{k}(x, y) \phi(y) d y
$$

Since it is assumed that $\alpha(x)$ is bounded away from zero for all $x$, the PDE in (2.1.2) satisfies a maximum principle and, therefore, $\widehat{k}$ is positive in $\Omega$. Thus, by the compactness of $\Omega$, there exists a positive constant $\bar{k}$, say, such that $\widehat{k} \geq \bar{k}>0$. Since $\Omega$ is compact, the function $\phi \equiv 1 \in P$. Thus, for $p=1$, there exists some $\chi>0$ such that

$$
\int_{\Omega} \widehat{k}(x, y) \phi(y) d y \geq \chi \phi>0
$$

By the bounds in (E.0.5), there exists some $\chi>0$ such that

$$
B \phi \geq \chi \phi
$$

and Theorem E.0.7 applies.

The simplicity of $\lambda$ and dominance over all other eigenvalues along with the uniqueness of $\phi$ requires that $B$ is a $u_{0}$-positive operator which is defined on page 194 in Krasnosel'skii and Zabresiko (1984) and stated as follows.

Definition E.0.8 ( $u_{0}$-positive operator) We say that the linear operator $A$ is $u_{0}$ positive if for every nonnegative $u \in P$ and a fixed nonzero element $u_{0} \in P$ there exist 
positive numbers $V(u), W(u)$ and a natural number $p$ such that

$$
V(u) u_{0} \leq A^{p} u \leq W(u) u_{0} .
$$

Since $\widehat{k} \geq \bar{k}>0$, the operator $B$ in (E.0.2) is strongly positive (see page 265 in Krasnosel'skii (1964b)). In particular, for every $\phi_{1} \in P$ there exists $v>0$ such that $B \phi_{1} \geq v\left\|\phi_{1}\right\|$.

Since $B$ in (E.0.2) is a continuous linear operator, it is bounded (Keener, 2000), so there exists a $w>0$ such that $B \phi_{1} \leq w\left\|\phi_{1}\right\|$. Now, we take $p=1$ and $u_{0}=\phi \equiv 1$ in Definition E.0.8, then there exists positive numbers $V\left(\phi_{1}\right)$ and $W\left(\phi_{1}\right)$ such that

$$
V\left(\phi_{1}\right) \phi \leq B \phi_{1} \leq W\left(\phi_{1}\right) \phi,
$$

where $V=v\left\|\phi_{1}\right\|$ and $W=w\left\|\phi_{1}\right\|$. Therefore, $B$ is a $u_{0}$-positive operator.

The desired results are now obtained from Theorems 2.10, 2.11, and 2.13 of Krasnosel'skii (1964a) which are summarized as the following theorem.

Theorem E.0.9 (A possess a dominant eigenpair) Let $A$ be a compact, linear, $u_{0}$-positive operator that leaves the reproducing cone $P$ invariant. Let $\phi_{0} \in P$ be a characteristic function of $A$ with corresponding characteristic value $\lambda_{0}$ :

$$
A \phi_{0}=\lambda_{0} \phi_{0}
$$

Then, $\lambda_{0}$ is a positive, simple characteristic value that is strictly larger in modulus than any other characteristic value of $A$, and its characteristic function is the only (up 
to constant multiple) nonnegative characteristic function of $A$. We refer to $\left(\lambda_{0}, \phi_{0}\right)$ as the dominant eigenpair.

With the existence of a dominant eigenpair, we now show that $\widehat{Q}_{l}$ possess a unique, positive fixed point. To motivate the necessary conditions for the existence and uniqueness of a positive fixed point of (4.2.5), we first consider a non-spatial model given by

$$
N_{t+1}=f\left(N_{t}\right),
$$

where $f$ is continuous, $f(0)=0, f^{\prime}(0)>1$, and $\lim _{n \rightarrow \infty} f^{\prime}(N)=0$. These conditions imply that $f$ must intersect the line $f(N)=N$ for some $N>0$ which proves the existence of a positive fixed point, $N^{*}$ of (E.0.7). For uniqueness, it is further required that $f$ is monotonically increasing and $f(N) / N$ decreases as $N$ increases. These assumptions imply that $f$ is a concave function, which is sufficient for the uniqueness of a positive fixed point.

As an example of the above theory, we assume that $f$ is given by the BevertonHolt function (1.2.5). The existence of a positive fixed point is clear since (1.2.5) satisfies F1 - F5. For uniqueness, we note that

$$
\lim _{N \rightarrow 0^{+}} \frac{f(N)}{N}=r \equiv f^{\prime}(0)>1
$$

so that $f(N) / N=1$ at one and only one point which implies $N^{*}$ is unique.

Existence and uniqueness of a positive fixed point for the spatial model (4.2.5) follow from analogous conditions to those for (E.0.7). For existence, it is required that $\widehat{Q}_{l}$ is continuous, $\widehat{Q}_{l}^{\prime}(0)$ exists with dominant eigenvalue larger than unity and its asymptotic derivative $\widehat{Q}_{l}^{\prime}(\infty)$ be the zero operator where the asymptotic derivative of $\widehat{Q}_{l}$ is defined as follows (see page 241 in Krasnosel'skii (1964a)). 
Definition E.0.10 (Asymptotic derivative) The asymptotic derivative of $\widehat{Q}_{l}$ is the compact, linear integral operator given by

$$
\widehat{Q}_{l}^{\prime}(\infty)[\phi]=\int_{\Omega} \lim _{N \rightarrow \infty} \frac{\widehat{k}(x, y) f(N(y) ; y)}{N} \phi(y) d y .
$$

The asymptotic derivative of $\widehat{Q}_{l}$ with $f$ given by $(1.2 .5)$ is

$$
\widehat{Q}_{l}^{\prime}(\infty)[\phi]=\int_{\Omega} \widehat{k}(x, y) \lim _{N \rightarrow \infty} \frac{r}{1+(r-1) N} \phi(y) d y=0,
$$

which is the zero or trivial operator. The existence of a positive fixed point of (4.2.5) then follows from Theorem 7.5, page 246 in Krasnosel'skii (1964a), which is stated as follows.

Theorem E.0.11 (Existence of positive fixed-point) Under the assumptions of this section, the equation $\widehat{Q}_{l}[N]=N$ has at least one nonnegative, non-identically zero solution if either $\lambda<1<\lambda_{\infty}$ or $\lambda_{\infty}<1<\lambda$, where $\lambda_{\infty}$ is the dominant eigenvalue of the asymptotic derivative.

Since the asymptotic derivative is given by the zero operator, $\lambda_{\infty}=0$, the above theorem applies. Because $\widehat{k}$ is nonnegative, the monotonicity and concavity of $\widehat{Q}_{l}$ follow from the properties of $f$, thus, $\widehat{Q}_{l}$ is a monotonic, concave operator. The uniqueness and global stability of the positive fixed point then follow from the results on pages 248-251 of Krasnosel'skii (1964a). 


\section{Appendix $\mathrm{F}$}

\section{Numerical Methods}

An integrodifference equation is an integral equation of the form

$$
N_{t+1}(x)=\int_{\Omega} k(x, y) f\left(N_{t}(y) ; y\right) d y
$$

We assume that the domain is finite and, without loss of generality, take $\Omega=\left[-\frac{L}{2}, \frac{L}{2}\right]$. Numerical methods for equation (F.0.1) largely depend on the properties of the dispersal kernel. For the purposes of the work in this thesis, we consider the following two cases.

\section{Case 1: Homogeneous Dispersal}

We assume that individuals are dispersing in a one-dimensional, homogeneous landscape so that dispersal depends only on distance; i.e., $k(x, y)=\tilde{k}(x-y)$. In this case, equation (F.0.1) may be written as

$$
N_{t+1}(x)=\int_{-\frac{L}{2}}^{\frac{L}{2}} \tilde{k}(x-y) f\left(N_{t}(y) ; y\right) d y:=f * k(x),
$$

a convolution integral. James Powell designed an efficient method to determine $N_{t+1}$ 
without directly evaluating the integral; see Powell (2009). This method utilizes the fact that the Fourier transform of a convolution is the product of Fourier transforms. To determine $N_{t+1}$ the following procedure may be used:

- calculate the Fourier transform $\hat{f}$ of $f$ and $\hat{k}$ of $k$;

- calculate the product of the transforms and note that $\widehat{f * k}=\frac{2 L}{n} \hat{f} \hat{k}$ (the factor of $\frac{2 L}{n}$ is explained below);

- apply the inverse transform to $\widehat{f * k}$ and obtain $f * k$.

Fourier transforms can be computed quickly in MATLAB using the Fast Fourier transform (FFT). FFT assumes that $\Omega$ is an interval of length one and that boundary conditions are periodic. FFT also scales the resulting product of transforms for by a factor of $n$; i.e., $\widehat{f * k}=n \hat{f} \hat{k}$. To circumvent periodic boundary conditions, we define the computational domain $\widehat{\Omega}=[-L, L]$ and set $f=0$ on $\widehat{\Omega} \backslash \Omega$. One also must multiply the product of transforms by $\frac{2 L}{n}$ to scale the interval to length $2 L$ and divide by $n$ to obtain the desired result; i.e., $\widehat{f * k}=\hat{f} \hat{k}$. Note that $\frac{2 L}{n}=\Delta x$, the discrete step size. Note also that an assumption of the FFT is that $n$ is a power of 2 ; for all simulations, we choose $n=2^{14}$. Finally, we note that $L$ was set to 200 for all simulations.

\section{Case 2: Heterogeneous Dispersal}

We assume that individuals are dispersing in a one-dimensional landscape that is periodically varying with good patches of length $l_{1}$ and bad patches of length $l_{2}$. Furthermore, we assume that dispersal is patch-dependent. In this case, the integral is no longer a convolution as the kernel is now dependent on source location $(y)$ and we can not make use of the FFT. We discretize $\Omega$ with constant step size given by $\Delta x=\frac{2 L}{n}$ where $n=2^{12}$. Note that, in the discretization process, one must ensure that interface points between good and bad patches are not grid points. 


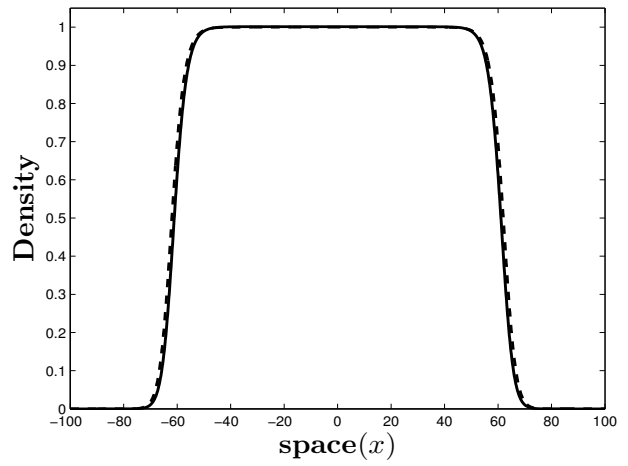

Figure F.1: Numerical simulation of equation (F.0.1) for 30 iterations obtained using the FFT (solid) and Simpson's rule (dashed) assuming the landscape is homogeneous; i.e., $l_{2}=0$. The domain was $\Omega=[-100,100]$ with $n=2^{12}$ and the initial condition was $N_{0}(0)=1$. Fixed parameter values are: $\alpha=1, \beta=0$, and $\nu=1$.

Since dispersal is patch-dependent, we generate dispersal kernels using the method described in Section 3.3. We note that, since kernels are shift invariant with period $l$, one only needs to generate kernels over a single period (e.g. $l=l_{1}+l_{2}$ ) and then shift the kernels accordingly to obtain a kernel for each $y \in \Omega$. For each $x \in \Omega$, the integral in (F.0.1) is evaluated using standard techniques; e.g., Simpson's Rule.

In the case that $l_{2}=0$, the periodic landscape simplifies to a homogeneous landscape. In this case, we can check the accuracy of our routine by comparing simulations with those obtained using the FFT. As an illustration, we simulated equation (F.0.1) using the FFT and Simpson's rule assuming and $n=2^{12}$. We observe from Figure F.1 that the two methods agree rather well. 


\section{Bibliography}

Anulewicz, A.C., McCullough, D.G., Cappaert, D.L.: Emerald ash borer (Agrilus planipennis) density and canopy dieback in three North American ash species. Arboriculture and Urban Forestry 33, 338-349 (2007)

Anulewicz, A.C., McCullough, D.G., Cappaert, D.L., Poland, T.M.: Host range of the emerald ash borer (Agrilus planipennis fairmaire) (coleoptera: Buprestidae) in North America: Results of multiple-choice field experiments. Environmental Entomology pp. 230-241 (2008)

Anulewicz, A.C., McCullough, D.G., Miller, D.L.: Oviposition and development of emerald ash borer (Agrilus planipennis) (coleoptera: Buprestidae) on hosts and potential hosts in no-choice bioassays. The Great Lakes Entomologist 39, 99-112 (2006)

van den Bosch, F., Metz, J.A.J., Diekmann, O.: The velocity of spatial population expansion. Journal of Mathematical Biology 28, 529-565 (1990)

Bossenbroek, J.M., Kraft, C.E., Nekola, J.C.: Prediction of long-distance dispersal using gravity models: Zebra mussel invasion of inland lakes. Ecological Applications 11, 1778-1788 (2001) 
Bowler, D., Benton, T.: Causes and consequences of animal dispersal strategies: Relating individual behaviour to spatial dynamics. Biological Reviews 80, 205-225 $(2005)$

Brown, K.J., Cosner, C., Fleckinger, J.: Principle eigenvalues for problems with indefinite weight functions on $\mathbb{R}^{n}$. Proceedings of the American Mathematical Society 109, $147-155(1990)$

Cantrell, R.S., Cosner, C.: Spatial Ecology via Reaction-Diffusion Equations. Mathematical and Computational Biology. Wiley (2003)

Cantrell, R.S., Cosner, C., Fagan, W.F.: The implications of model formulation when transitioning from spatial to landscape ecology. Mathematical Biosciences and Engineering 9, $27-60(2012)$

Cappaert, D., McCullough, D., Polland, T., Siegert, N.: Emerald ash borer in North America: A research and regulatory challenge. American Entomoloist 51, 152-165 (2005)

Chapman, D., Dytham, C., Oxford, G.: Landscape and fine-scale movements of a leaf beetle: The importance of boundary behaviour. Population Ecology 154, 55-64 (2007)

Clark, J.S.: Why trees migrate so fast: Confronting theory with dispersal biology and the paleorecord. The American Naturalist 152, 204-224 (1998)

Clark, J.S., Lewis, M., Horvath, L.: Invasion by extremes: Population spread with variation in dispersal and reproduction. The American Naturalist 157, 537-554 (2001) 
Conradt, L., Bodworth, E., Roper, T.J., Thomas, C.D.: Non-random dispersal in the butterfly Maniola jurtina: Implications for metapopulation models. Proceedings of the Royal Society of London B 267, 1505-1510 (2000)

Conradt, L., Roper, T.J., Thomas, C.D.: Dispersal behaviour of individuals in metapopulations of two british butterflies. OIKOS 95, 416-424 (2001)

Crosthwaite, J.C., Sobek, S., Lyons, D.B., Bernards, M.A., Sinclair, B.J.: The overwintering physiology of the emerald ash borer, Agrilus planipennis fairmaire (coleoptera: Buprestidae). Journal of Insect Physiology 57, 166-173 (2011)

Dewhirst, S., Lutscher, F.: Dispersal in heterogeneous habitats: Thresholds, spatial scales, and approximate rates of spread. Ecology 90, 1338 - 1345 (2009)

Fagan, W.F., Lutscher, F.: Average dispersal success: Linking home range, dispersal, and metapopulation dynamics to reserve design. Ecological Applications 16, 820$828(2006)$

Fahrig, L.: Effect of habitat fragmentation on the extinction threshold: A synthesis. Ecological Applications 12, 346-353 (2002)

Fisher, R.A.: The wave of advance of advantageous genes. Annals of Eugenics 7, 355-369 (1937)

Fryxell, J.F.: Predictive modelling of patch use by terrestrial herbivores. In Dynamics of Foraging Resource Ecology: Spatial and Temporal. Springer (2008)

Gillis, E.A., Nams, V.O.: How red-backed voles find habitat patches. Canadian Journal of Zoology 76, 791-794 (1998) 
Houtan, K.S.V., Pimm, S.L., Halley, J.M., Bierregaard, R.O., Jr., Lovejoy, T.E.: Dispersal of amazonian birds in continuous and fragmented forest. Ecology Letters 10, 219-229 (2007)

Hsu, S.B., Zhao, X.Q.: Spreading speeds and traveling waves for non-monotone integrodifference equations. SIAM Journal of Mathematical Analysis 40, 776-789 (2008)

Iman, R.L., Conover, W.J.: Small sample sensitivity analysis techniques for computer models, with an application to risk assessment. Communications in Statistics Theory and Methods 9, 1749-1842 (1980)

Kawasaki, K., Asano, K., Shigesada, N.: Impact of directed movements on invasive spread in periodic patchy environments. Bulletin of Mathematical Biology 74, $1448-1467(2012)$

Kawasaki, K., Shigesada, N.: An integrodifference model for biological invasions in a periodically fragmented environment. Japan Journal of Industrial and Applied Mathematics 24, 3-15 (2007)

Keener, J.P.: Principles of Applied Mathematics: Transformation and Approximation. Westview Press, Massachusetts (2000)

Kevorkian, J.: Partial Differential Equations: Analytical Solution Techniques. Chapman and Hall, London, UK (1990)

Kot, M., Lewis, M., van den Driessche, P.: Dispersal data and the spread of invading organisms. Ecology 77, 2027-2042 (1996) 
Kot, M., Medlock, J., Reluga, T., Wilson, D.B.: Stochasticity, invasions, and branching random walks. Theoretical Population Biology 66, 175-184 (2004)

Kot, M., Schaffer, W.M.: Discrete-time growth models. Mathematical Biosciences 80, 109-136 (1986)

Krasnosel'skii, M.: Positive Solutions of Operator Equations. Noordhoff, Groningen, The Netherlands (1964a)

Krasnosel'skii, M.: Topological Methods in the Theory of Nonlinear Integral Equations. Pergamon Press, Oxford (1964b)

Krasnosel'skii, M., Zabresiko, P.P.: Geometrical Methods of Nonlinear Analysis. Springer-Verlag, Germany (1984)

Krein, M.G., Rutman, M.A.: Linear operators which leave a cone in banach space invariant. American Mathematical Society Translations 26 (1950)

Lax, P.: Functional Analysis. John Wiley and Sons, New York, NY (2002)

Liebhold, A., Tobin, P.: Population ecology of insect invasions and their management. Annual Review of Entomology 53, 387-408 (2008)

Lockwood, D.R., Hastings, A., Botsford, L.W.: The effects of dispersal patterns on marine reserves: Does the tail wag the dog? Theoretical Population Biology 61, 297-309 (2002)

Ludwig, D., Aronson, D., Weinberger, H.: Spatial patterning of the spruce budworm. Journal of Mathematical Biology 9, 217-258 (1979)

Lutscher, F.: A short note on short distance dispersal events. Bulletin of Mathematical Biology 69, 1615-1630 (2007) 
Lutscher, F., Lewis, M.: Spatially-explicit matrix models: A mathematical analysis of stage-structured integrodifference equations. Journal of Mathematical Biology 48, 293-324 (2004)

Lutscher, F., Nisbet, R., Pachepsky, E.: Population persistence in the face of advection. Theoretical Ecology 3, 271-284 (2010)

Lyons, D.B., de Groot, P., Jones, G.C., Scharbach, R.: Host selection by Agrilus planipennis (coleoptera: Buprestidae): Inferences from sticky-band trapping. The Canadian Entomologist 141, 40-52 (2009)

Maciel, G.A., Lutscher, F.: How how individual movement response to habitat edges affects population persistence and spatial spread. The American Naturalist (2013). DOI $10.1086 / 670661$

Magnus, W., Winkler, S.: Hill's Equation. N.20 in Interscience Tracts in Pure and Applied Mathematics. Interscience Publishers, New York (1966)

Marino, S., Hogue, I.B., Ray, C.J., Kirschner, D.E.: A methodology for performing global uncertainty and sensitivity analysis in systems biology. Journal of Theoretical Biology 254, 178-196 (2008)

Mastro, V., Lance, D., Reardon, R., (editors), G.P.: Emerald ash borer research and technology development meeting. USDA Forest Service, Pittsburgh, Pennsylvania (2007)

McCullough, D., Siegert, N.: Estimating potential emerald ash borer (coleoptera: Buprestidae) populations using ash inventory data. Journal of Economic Entomology 100, 1577-1586 (2007) 
McCullough, D.G., Mercader, R.J.: Evaluation of potential strategies to slow ash mortality (SLAM) caused by emerald ash borer (Agrilus planipennis): SLAM in an urban forest. International Journal of Pest Management 58, 9-23 (2012)

McCullough, D.G., Poland, T.M., Anulewicz, A.C., Cappaert, D.L.: Emerald ash borer (coleoptera: Buprestidae) attraction to stressed or baited ash trees. Environmental Entomology 38, 1668-1679 (2009)

Mercader, R.J., Siegert, N.W., Liebhold, A.M., McCullough, D.G.: Dispersal of the emerald ash borer, Agirlus planipennis, in newly-colonized sites. Agricultural and Forest Entomology pp. 421-424 (2009)

Mercader, R.J., Siegert, N.W., Liebhold, A.M., McCullough, D.G.: Influence of foraging behavior and host spatial distribution on the localized spread of the emerald ash borer (Agirlus planipennis). Population Ecology pp. 271-285 (2011a)

Mercader, R.J., Siegert, N.W., Liebhold, A.M., McCullough, D.G.: Simulating the effectiveness of three potential management options to slow the spread of emerald ash borer (Agirlus planipennis) populations in localized outlier sites. Canadian Journal of Forest Research pp. 254-264 (2011b)

Muirhead, J., Leung, B., van Overdijk, C., Kelly, D., Nandakumar, K., Marchant, K., MacIssac, H.: Modeling local and long-distance dispersal of invasive emerald ash borer Agrilus planipennis (coleoptera) in north america. Diversity and Distributions 12, 71-79 (2006)

Nagylaki, T.: Clines with variable migration. Genetics 83, 867-886 (1976)

Neubert, M., Kot, M., Lewis, M.: Dispersal and pattern formation in a discrete-time predatory-prey model. Theoretical Population Biology 48, 7-43 (1995) 
Ovaskainen, O., Cornell, S.J.: Biased movement at a boundary and conditional occupancy times for diffusion processes. Journal of Applied Probability 40, 557-580 $(2003)$

Pielou, E.C.: An Introduction to Mathematical Ecology. Wiley, New York (1969)

Poland, T., McCullough, D.: Emerald ash borer: Invasion of the Urban Forest and the Threat to North American's Ash Resource. Journal of Forestry 104, 118-124 (2006)

Powell, J.: Spatiotemporal Models in Ecology: An Introduction to Integrodifference Equations. Utah State University (2009)

Powell, J., Zimmermann, N.: Multiscale analysis of active seed dispersal contributes to resolving reid's paradox. Ecology 85, 490-506 (2004)

Prasad, A., Iverson, L., Peters, M., Bossenbroek, J., Matthews, S., Snydor, T.D., Schwartz, M.: Modeling the invasive emerald ash borer risk of spread using a spatially explicit cellular model. Landscape Ecology 25, 353-369 (2010)

Robbins, T.: Seed dispersal and biological invasions: A mathematical analysis. Ph.D. thesis, University of Utah, Salt Lake City, Utah (2004)

Rutledge, C.E., Keena, M.A.: Mating frequency and fecundity in the emerald ash borer Agrilus planipennis (coleoptera: Buprestidae). Annals of the Entomological Society of America 105, 66-72 (2012)

Schooley, R.L., Wiens, J.A.: Finding habitat patches and directional connectivity. OIKOS 102, 559-570 (2003) 
Schultz, C., Crone, E.: Old models explain new observations of butterfly movement at patch edges. Ecology 89, 2061-2067 (2008)

Sharov, A., Liebhold, A.: Model of slowing the spread of gypsy moth (lepidoptera: Lymantriidae) with a barrier zone. Ecological Applications 8, 1170-1179 (1998)

Shigesada, N., Kawasaki, K., Takeda, Y.: Modeling stratified diffusion in biological invasions. American Naturalist 146, 229-251 (1995)

Shigesada, N., Kawasaki, K., Teramoto, E.: Travelling periodic waves in heterogeneous environments. Theoretical Population Biology 30, 143-160 (1986)

Siegert, N., McCullough, D., Tluczek, A.: Two years under the bark: Towards understanding multiple-year development of emerald ash borer larvae. In: V. Mastro and L.R. Reardon and G. Parra (eds) Emerald ash borer and asian longhorned beetle research and technology development meeting. USDA Forest Service, Morgantown, Michigan (2006)

Siegert, N., McCullough, D., Williams, D., Fraser, I., Polland, T., Pierce, S.: Dispersal of Agrilus planipennis(coleoptera: Buprestidae) from discrete epicentres in two outlier sites. Environmental Entomology 39, 253-265 (2010)

Skellam, J.: Random Dispersal in theoretical populations. Biometrika 38, 196-218 (1951)

Thomas, C.D.: Dispersal and extinction in fragmented landscapes. Proceedings of the Royal Society of London B 267, 139-145 (2000)

Turchin, P.: Quantitative Analysis of Movement: Measuring and Modeling Popula- 
tion Redistribution in Animals and Plants. Sinauer Associates, Inc., Massachusetts (1998)

VanKirk, R.W.: Integrodifference models of biological growth and dispersal. Ph.D. thesis, University of Utah, Salt Lake City, Utah (1995)

VanKirk, R.W., Lewis, M.A.: Integrodifference models for persistence in fragmentated habitats. Bulletin of Mathematical Biology 59, 107-137 (1997)

VanKirk, R.W., Lewis, M.A.: Edge permeability and population persistence in isolated habitat patches. Natural Resource Modeling 12, 37 - 64 (1999)

Weinberger, H., Kohkichi, Kawasaki, Shigesada, N.: Spreading speeds of spatially periodic integrodifference models for populations with non-monotone recruitment functions. Journal of Mathematical Biology 57, 387 - 411 (2008)

Weinberger, H.F.: Long-time behavior of a class of biological models. SIAM Journal of Mathematical Analysis 13, 353-396 (1982)

Weinberger, H.F.: On spreading speeds and traveling waves for growth and migration models in a periodic habitat. Journal of Mathematical Biology 45, 511-548 (2002)

With, K.A.: The landscape ecology of invasive spread. Conservation Biology 16, 1192 $-1203(2002)$

Zabresiko, P.P., Koshelev, A., Krasnosel'skii, M., Mikhlin, S., Rakovshchik, L., Stet'senko, V.Y.: Integral equations - a reference text. Noordhoff International Publishing, Leyden, The Netherlands (1975)

Zhou, Y., Kot, M.: Discrete-time growth-dispersal models with shifting species ranges. Theoretical Ecology 4, 13-25 (2011) 
Zollner, P.A.: Comparing the landscape level perceptual abilities of forest sciurids in fragmented agricultural landscapes. Landscape Ecology 15, 523-533 (2000) 